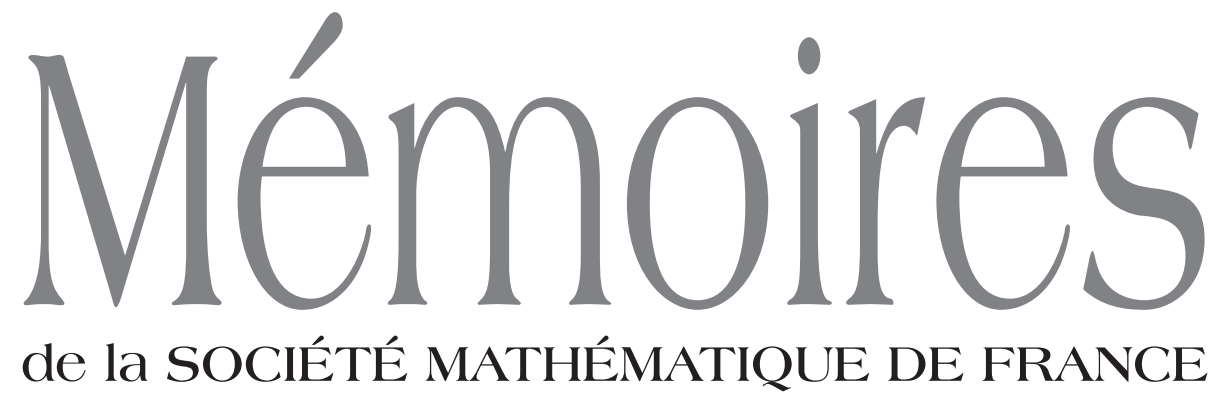

Numéro 117 Nouvelle série
CREATION OF FERMIONS BY ROTATING CHARGED BLACK HOLES

$\begin{array}{llll}2 & 0 & 0 & 9\end{array}$

SOCIÉTÉ MATHÉMATIQUE DE FRANCE

Publié avec le concours du Centre National de la Recherche Scientifique 


\title{
Comité de rédaction
}

\author{
Jean BARGE \\ Emmanuel BREUILLARD \\ Gérard BESSON \\ Antoine CHAMBERT-LOIR \\ Jean-François DAT \\ Raphaël KRIKORIAN (dir.) \\ Charles FAVRE \\ Daniel HUYBRECHTS \\ Yves LE JAN \\ Laure SAINT-RAYMOND \\ Wilhem SCHLAG
}

\section{Diffusion}

Maison de la SMF

Case 916 - Luminy

13288 Marseille Cedex 9

France

smf@smf .univ-mrs.fr
Hindustan Book Agency

O-131, The Shopping Mall

Arjun Marg, DLF Phase 1

Gurgaon 122002, Haryana

Inde
AMS

P.O. Box 6248

Providence RI 02940

USA

WwW . ams . org

\section{Tarifs}

Vente au numéro : $28 €(\$ 42)$

Abonnement Europe : $255 €$, hors Europe : $290 €(\$ 435)$

Des conditions spéciales sont accordées aux membres de la SMF.

\section{Secrétariat : Nathalie Christiaën}

Mémoires de la SMF

Société Mathématique de France

Institut Henri Poincaré, 11, rue Pierre et Marie Curie

75231 Paris Cedex 05, France

Tél : (33) 0144276799 • Fax : (33) 0140469096

revues@smf.ens.fr • http://smf.emath.fr/

(C) Société Mathématique de France 2009

Tous droits réservés (article L 122-4 du Code de la propriété intellectuelle). Toute représentation ou reproduction intégrale ou partielle faite sans le consentement de l'éditeur est illicite. Cette représentation ou reproduction par quelque procédé que ce soit constituerait une contrefaçon sanctionnée par les articles $L$ 335-2 et suivants du CPI.

ISSN 0249-633-X

ISBN 978-285629-284-6

Directrice de la publication : Aline BONAMI 


\title{
CREATION OF FERMIONS BY ROTATING CHARGED BLACK HOLES
}

\author{
Dietrich HÄFNER
}

Société Mathématique de France 2009

Publié avec le concours du Centre National de la Recherche Scientifique 
Dietrich HÄFNER

Université Bordeaux 1, Institut de Mathématiques de Bordeaux, 351 cours de la Libération, 33405 Talence CEDEX (France).

E-mail : Dietrich.Hafner@math.u-bordeaux1.fr

2000 Mathematics Subject Classification. - 35P25，35Q75，58J45，83C47，83C57, $83 \mathrm{C} 60$.

Key words and phrases. - General relativity, Kerr-Newman metric, Quantum field theory, Hawking effect, Dirac equation, Scattering theory, Characteristic Cauchy problem. 


\title{
CREATION OF FERMIONS BY ROTATING CHARGED BLACK HOLES
}

\author{
Dietrich HÄFNER
}

\begin{abstract}
This work is devoted to the mathematical study of the Hawking effect for fermions in the setting of the collapse of a rotating charged star. We show that an observer who is located far away from the star and at rest with respect to the Boyer Lindquist coordinates observes the emergence of a thermal state when his proper time goes to infinity. We first introduce a model of the collapse of the star. We suppose that the space-time outside the star is given by the Kerr-Newman metric. The assumptions on the asymptotic behavior of the surface of the star are inspired by the asymptotic behavior of certain timelike geodesics in the Kerr-Newman metric. The Dirac equation is then written using coordinates and a Newman-Penrose tetrad which are adapted to the collapse. This coordinate system and tetrad are based on the so called simple null geodesics. The quantization of Dirac fields in a globally hyperbolic space-time is described. We formulate and prove a theorem about the Hawking effect in this setting. The proof of the theorem contains a minimal velocity estimate for Dirac fields that is slightly stronger than the usual ones and an existence and uniqueness result for solutions of a characteristic Cauchy problem for Dirac fields in the Kerr-Newman space-time. In an appendix we construct explicitly a Penrose compactification of block $I$ of the Kerr-Newman space-time based on simple null geodesics.
\end{abstract}

\section{Résumé (Création de fermions par des trous noirs chargés en rotation)}

Ce travail est consacré à l'étude mathématique de l'effet Hawking pour des fermions dans le cadre de l'effondrement d'une étoile chargée en rotation. On démontre qu'un observateur localisé loin de l'étoile et au repos par rapport aux coordonnées de BoyerLindquist observe l'émergence d'un état thermal quand son temps propre tend vers l'infini. On introduit d'abord un modèle de l'effondrement de l'étoile. On suppose que l'espace-temps à l'extérieur de l'étoile est donné par la métrique de Kerr-Newman. Les hypothèses sur le comportement asymptotique de la surface de l'étoile sont inspirées par le comportement asymptotique de certaines géodésiques de type temps dans la métrique de Kerr-Newman. L'équation de Dirac est alors écrite en utilisant 
des coordonnées et une tétrade de Newman-Penrose adaptées à l'effondrement. Ce système de coordonnées et cette tétrade sont basés sur des géodésiques qu'on appelle des géodésiques simples isotropes. La quantification des champs de Dirac dans un espace-temps globalement hyperbolique est décrite. On formule un théorème sur l'effet Hawking dans ce cadre. La preuve du théorème contient une estimation de vitesse minimale pour les champs de Dirac légèrement plus forte que les estimations usuelles ainsi qu'un résultat d'existence et d'unicité pour les solutions d'un problème caractéristique pour les champs de Dirac dans l'espace-temps de Kerr-Newman. Dans un appendice, nous construisons explicitement la compactification de Penrose du bloc $I$ de l'espace-temps de Kerr-Newman qui est basée sur les géodésiques simples isotropes. 


\section{CONTENTS}

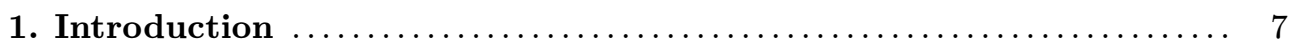

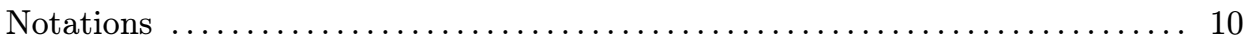

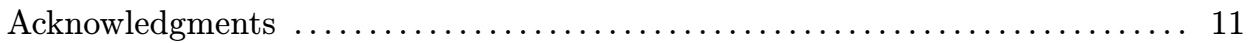

2. Strategy of the proof and

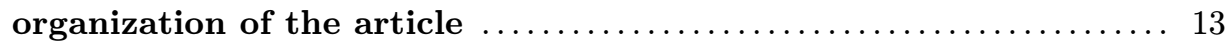

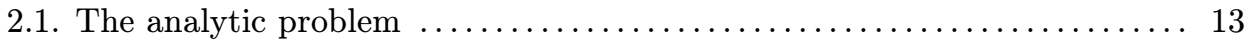

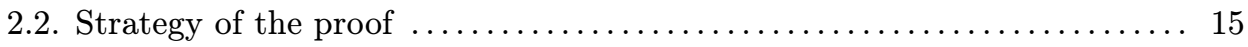

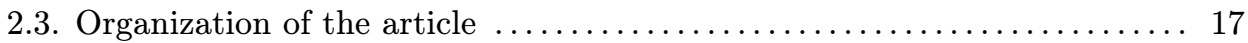

3. The model of the collapsing star $\ldots \ldots \ldots \ldots \ldots \ldots \ldots \ldots \ldots \ldots \ldots \ldots$

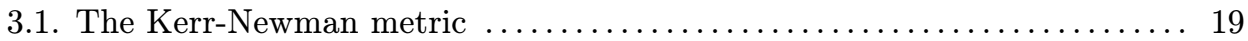

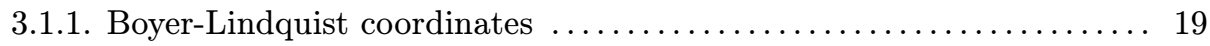

3.1.2. Some remarks about geodesics in the Kerr-Newman space-time .... 21

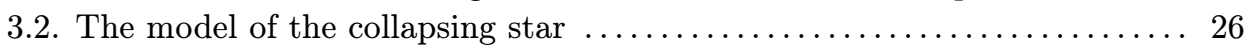

3.2.1. Timelike geodesics with $L=Q=\widetilde{E}=0 \ldots \ldots \ldots \ldots \ldots \ldots \ldots$

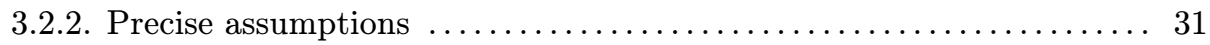

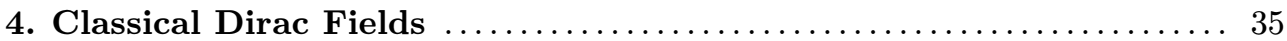

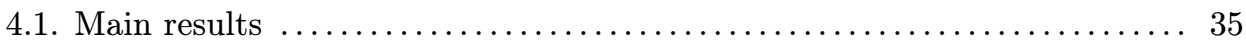

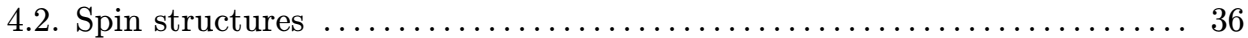

4.3. The Dirac equation and the Newman-Penrose formalism $\ldots \ldots \ldots \ldots \ldots 37$

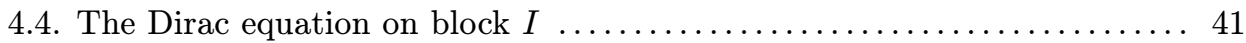

4.4.1. A new Newman-Penrose tetrad ....................... 41

4.4.2. The new expression of the Dirac equation $\ldots \ldots \ldots \ldots \ldots \ldots \ldots \ldots 4$

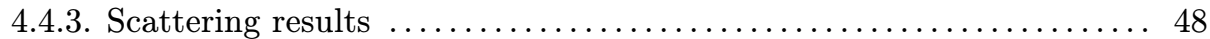

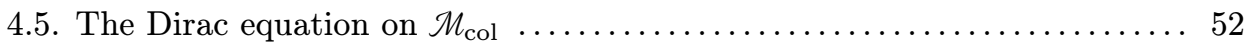

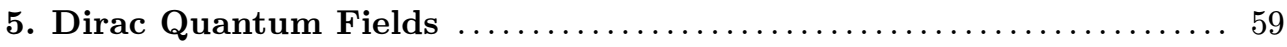

5.1 . Second Quantization of Dirac Fields $\ldots \ldots \ldots \ldots \ldots \ldots \ldots \ldots \ldots \ldots \ldots \ldots$

5.2 . Quantization in a globally hyperbolic space-time $\ldots \ldots \ldots \ldots \ldots \ldots \ldots 63$

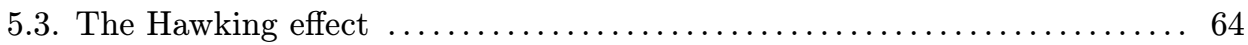

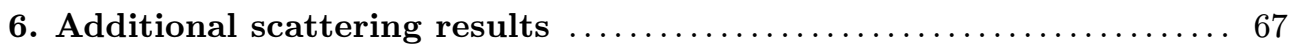

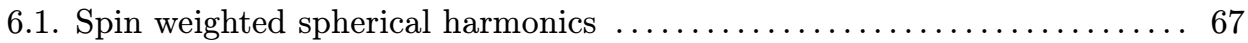




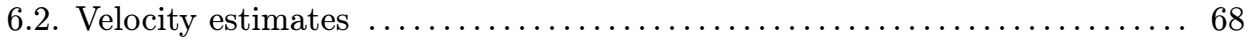

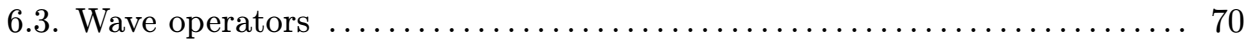

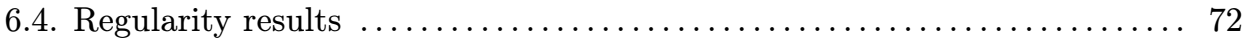

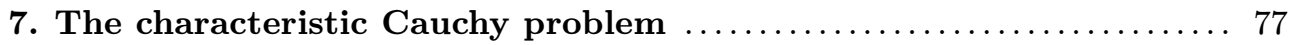

7.1. Main results ......................................... 77

7.2. The Cauchy problem with data on a lipschitz space-like hypersurface $\ldots 81$

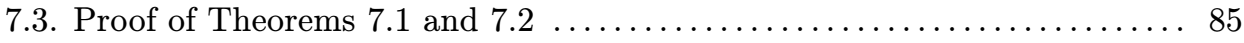

7.4. The characteristic Cauchy problem on $\mathcal{M}_{\text {col }} \ldots \ldots \ldots \ldots \ldots \ldots \ldots \ldots$

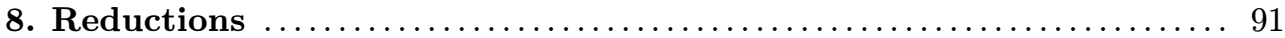

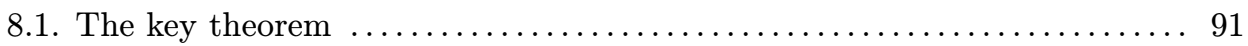

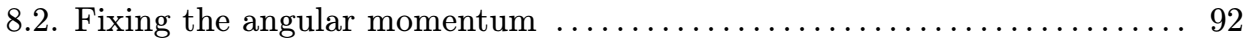

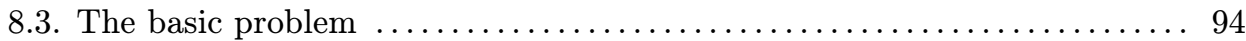

8.4. The mixed problem for the asymptotic dynamics $\ldots \ldots \ldots \ldots \ldots \ldots \ldots 96$

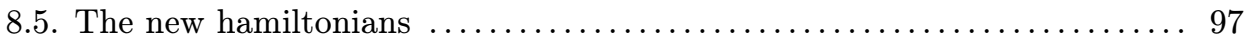

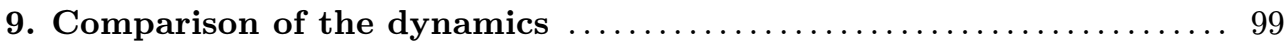

9.1. Comparison of the characteristic data $\ldots \ldots \ldots \ldots \ldots \ldots \ldots \ldots \ldots \ldots \ldots$

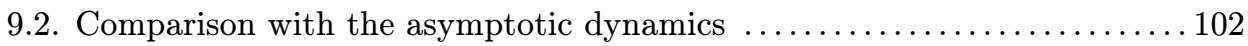

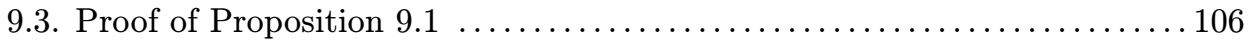

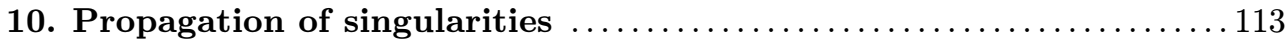

10.1. The geometric optics approximation and its properties $\ldots \ldots \ldots \ldots \ldots 115$

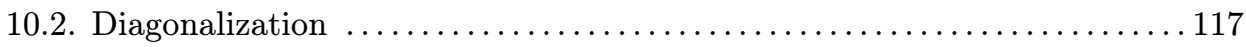

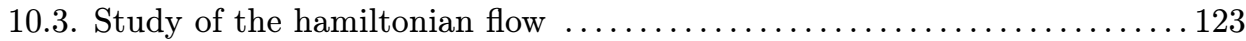

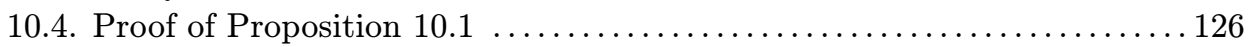

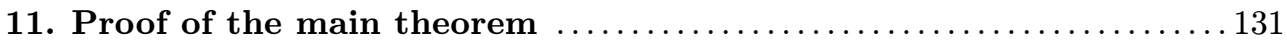

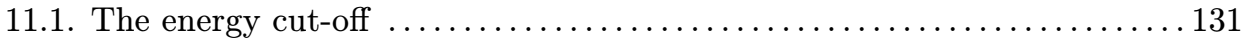

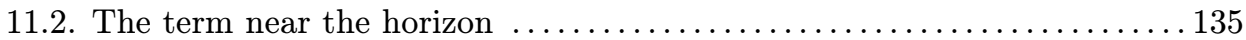

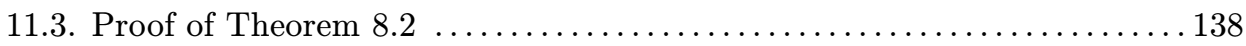

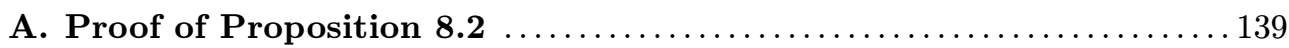

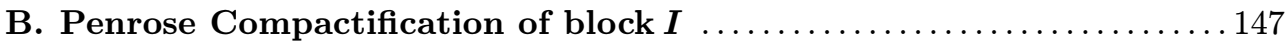

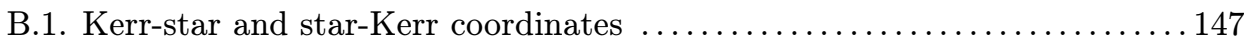

B.2. Kruskal-Boyer-Lindquist coordinates $\ldots \ldots \ldots \ldots \ldots \ldots \ldots \ldots \ldots \ldots \ldots \ldots \ldots$

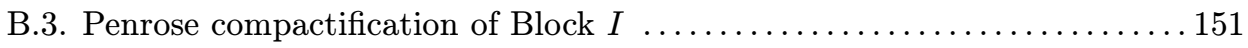

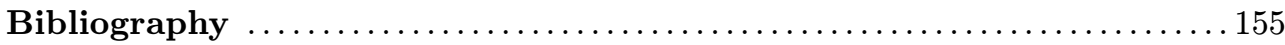




\section{CHAPTER 1}

\section{INTRODUCTION}

It was in 1975 that S.W. Hawking published his famous paper about the creation of particles by black holes (see [30]). Later this effect was analyzed by other authors in more detail (see e.g. [47]) and we can say that the effect was well understood from a physical point of view at the end of the 1970's. From a mathematical point of view, however, fundamental questions linked to the Hawking radiation such as scattering theory for field equations on black hole space-times had not been addressed at that time.

In the early 1980's Dimock and Kay started a research programme concerning scattering theory on curved space-times. They obtained an asymptotic completeness result for classical and quantum massless scalar fields on the Schwarzschild metric (see [21]-[20]). Their work was pushed further by Alain Bachelot in the 1990's. He showed asymptotic completeness for Maxwell and Klein-Gordon fields (see [1], [2]) and gave a mathematically precise description of the Hawking effect (see [3]-[5]) in the spherically symmetric case. Meanwhile other authors contributed to the subject such as Nicolas [38], Jin [33] and Melnyk [36], [37]. All these works deal with the spherically symmetric case.

The more realistic case of a rotating black hole is more difficult. In the spherically symmetric case, the study of a field equation can be reduced to the study of a $1+1$ dimensional equation with potential. In the Kerr case this reduction is no longer possible and the methods used in the papers cited so far do not apply. A paper by De Bièvre, Hislop, Sigal using different methods appeared in 1992 (see [10]). By means of a Mourre estimate they show asymptotic completeness for the wave equation on non-compact Riemannian manifolds; possible applications are therefore static situations such as the Schwarzschild case, which they treat, but the Kerr geometry is not even stationary. In this context we also mention the paper of Daudé about the Dirac equation in the Reissner-Nordström metric (see [14]). A complete scattering theory for the wave equation on stationary, asymptotically flat space-times, was obtained by the author in 2001 (see [27]). To our knowledge the first asymptotic completeness result in the Kerr case was obtained by the author in [28], for the non superradiant 
modes of the Klein-Gordon field. The first complete scattering theory for a field equation in the Kerr metric was obtained by Nicolas and the author in [29] for massless Dirac fields. This result was generalized by Daudé in [15] to the massive charged Dirac field in the Kerr-Newman metric. All these papers use Mourre theory.

The aim of the present paper is to give a mathematically precise description of the Hawking effect for spin $\frac{1}{2}$ fields in the setting of the collapse of a rotating charged star. We show that an observer who is located far away from the black hole and at rest with respect to the Boyer-Lindquist coordinates observes the emergence of a thermal state when his proper time $t$ goes to infinity. Let us give an idea of the theorem describing the effect. Let $r_{*}$ be the Regge-Wheeler coordinate. We suppose that the boundary of the star is described by $r_{*}=z(t, \theta)$. The space-time is then given by

$$
\mathcal{M}_{\mathrm{col}}=\bigcup_{t} \Sigma_{t}^{\mathrm{col}}, \quad \Sigma_{t}^{\mathrm{col}}=\left\{\left(t, r_{*}, \omega\right) \in \mathbb{R}_{t} \times \mathbb{R}_{r_{*}} \times S^{2} ; r_{*} \geq z(t, \theta)\right\} .
$$

The typical asymptotic behavior of $z(t, \theta)$ is $\left(\kappa_{+}>0\right)$ :

$$
z(t, \theta)=-t-A(\theta) \mathrm{e}^{-2 \kappa+t}+B(\theta)+\theta\left(\mathrm{e}^{-4 \kappa_{+} t}\right), \quad t \rightarrow \infty .
$$

Let $\mathscr{H}_{t}=L^{2}\left(\left(\Sigma_{t}^{\mathrm{col}}, \mathrm{dVol}\right) ; \mathbb{C}^{4}\right)$. The Dirac equation can be written as

$$
\partial_{t} \Psi=i D_{t} \Psi+\text { boundary condition. }
$$

We will put a MIT boundary condition on the surface of the star. The evolution of the Dirac field is then described by an isometric propagator $U(t, s): \mathscr{H}_{s} \rightarrow \mathscr{H}_{t}$. The Dirac equation on the whole exterior Kerr-Newman space-time $\mathcal{M}_{\mathrm{BH}}$ will be written as

$$
\partial_{t} \Psi=i \not D \Psi \text {. }
$$

Here $\not D$ is a selfadjoint operator on $\mathcal{H}=L^{2}\left(\left(\mathbb{R}_{r_{*}} \times S^{2}, \mathrm{~d} r_{*} \mathrm{~d} \omega\right) ; \mathbb{C}^{4}\right)$. There exists an asymptotic velocity operator $P^{ \pm}$such that for all continuous functions $J$ with $\lim _{|x| \rightarrow \infty} J(x)=0$ we have

$$
J\left(P^{ \pm}\right)=s-\lim _{t \rightarrow \pm \infty} \mathrm{e}^{-i t \not D} J\left(\frac{r_{*}}{t}\right) \mathrm{e}^{i t \not D} .
$$

Let $\mathcal{U}_{\text {col }}\left(\mathcal{M}_{\mathrm{col}}\right)$ (resp. $\left.\mathcal{U}_{\mathrm{BH}}\left(\mathcal{M}_{\mathrm{BH}}\right)\right)$ be the algebras of observables outside the collapsing body (on the space-time describing the eternal black hole) generated by $\Psi_{\text {col }}^{*}\left(\Phi_{1}\right) \Psi_{\text {col }}\left(\Phi_{2}\right)$ (resp. $\Psi_{\mathrm{BH}}^{*}\left(\Phi_{1}\right) \Psi_{\mathrm{BH}}\left(\Phi_{2}\right)$ ). Here $\Psi_{\text {col }}(\Phi)$ (resp. $\Psi_{\mathrm{BH}}(\Phi)$ ) are the quantum spin fields on $\mathcal{M}_{\text {col }}\left(\right.$ resp. $\left.\mathcal{M}_{\mathrm{BH}}\right)$. Let $\omega_{\text {col }}$ be a vacuum state on $\mathcal{U}_{\text {col }}\left(\mathcal{M}_{\text {col }}\right)$; $\omega_{\text {vac }}$ a vacuum state on $\mathcal{U}_{\mathrm{BH}}\left(\mathcal{M}_{\mathrm{BH}}\right)$ and $\omega_{\mathrm{Haw}}^{\eta, \sigma}$ be a KMS-state on $\mathcal{U}_{\mathrm{BH}}\left(\mathcal{M}_{\mathrm{BH}}\right)$ with inverse temperature $\sigma>0$ and chemical potential $\mu=\mathrm{e}^{\sigma \eta}$ (see Chapter 5 for details). For a function $\Phi \in C_{0}^{\infty}\left(\mathcal{M}_{\mathrm{BH}}\right)$ we define

$$
\Phi^{T}\left(t, r_{*}, \omega\right)=\Phi\left(t-T, r_{*}, \omega\right) .
$$

The theorem about the Hawking effect is:

TheOREM 1.1 (Hawking effect). - Let

$$
\Phi_{j} \in\left(C_{0}^{\infty}\left(\mathcal{M}_{\mathrm{col}}\right)\right)^{4}, \quad j=1,2 .
$$


Then we have

$$
\begin{aligned}
& \lim _{T \rightarrow \infty} \omega_{\text {col }}\left(\Psi_{\text {col }}^{*}\left(\Phi_{1}^{T}\right) \Psi_{\mathrm{col}}\left(\Phi_{2}^{T}\right)\right) \\
& =\omega_{\text {Haw }}^{\eta, \sigma}\left(\Psi_{\mathrm{BH}}^{*}\left(\mathbf{1}_{\mathbb{R}^{+}}\left(P^{-}\right) \Phi_{1}\right) \Psi_{\mathrm{BH}}\left(\mathbf{1}_{\mathbb{R}^{+}}\left(P^{-}\right) \Phi_{2}\right)\right) \\
& \quad+\omega_{\mathrm{vac}}\left(\Psi_{\mathrm{BH}}^{*}\left(\mathbf{1}_{\mathbb{R}^{-}}\left(P^{-}\right) \Phi_{1}\right) \Psi_{\mathrm{BH}}\left(\mathbf{1}_{\mathbb{R}^{-}}\left(P^{-}\right) \Phi_{2}\right)\right), \\
& T_{\text {Haw }}=\frac{1}{\sigma}=\frac{\kappa_{+}}{2 \pi}, \quad \mu=\mathrm{e}^{\sigma \eta}, \quad \eta=\frac{q Q r_{+}}{r_{+}^{2}+a^{2}}+\frac{a D_{\varphi}}{r_{+}^{2}+a^{2}} .
\end{aligned}
$$

Here $q$ is the charge of the field, $Q$ the charge of the black hole, $a$ the angular momentum per unit mass of the black hole, $r_{+}=M+\sqrt{M^{2}-\left(a^{2}+Q^{2}\right)}$ defines the outer event horizon and $\kappa_{+}$is the surface gravity of this horizon. The interpretation of (1.2) is the following. We start with a vacuum state which we evolve in the proper time of an observer at rest with respect to the Boyer Lindquist coordinates. The limit when the proper time of this observer goes to infinity is a thermal state coming from the event horizon in formation and a vacuum state coming from infinity as expressed on the R.H.S of (1.2). The Hawking effect is often interpreted in terms of particles, the antiparticle falling into the black hole and the particle escaping to infinity. From our point of view this interpretation is somewhat misleading. The effect really comes from an infinite Doppler effect and the mixing of positive and negative frequencies. To explain this a little bit more we describe the analytic problem behind the effect. Let $f\left(r_{*}, \omega\right) \in C_{0}^{\infty}\left(\mathbb{R} \times S^{2}\right)$. The key result about the Hawking effect is

$$
\begin{aligned}
& \lim _{T \rightarrow \infty}\left\|\mathbf{1}_{[0, \infty)}\left(\not D_{0}\right) U(0, T) f\right\|_{0}^{2} \\
& \quad=\left\langle\mathbf{1}_{\mathbb{R}^{+}}\left(P^{-}\right) f, \mu \mathrm{e}^{\sigma \not D}\left(1+\mu \mathrm{e}^{\sigma \not D}\right)^{-1} \mathbf{1}_{\mathbb{R}^{+}}\left(P^{-}\right) f\right\rangle+\left\|\mathbf{1}_{[0, \infty)}(\not D) \mathbf{1}_{\mathbb{R}^{-}}\left(P^{-}\right) f\right\|^{2},
\end{aligned}
$$

where $\mu, \eta, \sigma$ are as in the above theorem. Equation (1.3) implies (1.2).

The term on the L.H.S. comes from the vacuum state we consider. We have to project on the positive frequency solutions (see Chapter 5 for details). Note that we consider in (1.3) the time reversed evolution. This comes from the quantization procedure. When time becomes large the solution hits the surface of the star at a point closer and closer to the future event horizon. Figure 1 shows the situation for an asymptotic comparison dynamics, which satisfies Huygens' principle. For this asymptotic comparison dynamics the support of the solution concentrates more and more when time becomes large, which means that the frequency increases. The consequence of the change in frequency is that the system does not stay in the vacuum state.

We conclude this introduction with some comments on the boson case which we do not treat in this paper. This case is more difficult because of the superradiance phenomenon. There exists no positive conserved energy for the wave equation in block $I$ of the Kerr metric. This is linked to the fact that the Kerr metric is not stationary outside the black hole. Because of the difficulty linked to superradiance, there is at present no complete scattering theory for the wave equation on the Kerr metric, a necessary prerequisite for the mathematical description of the Hawking 


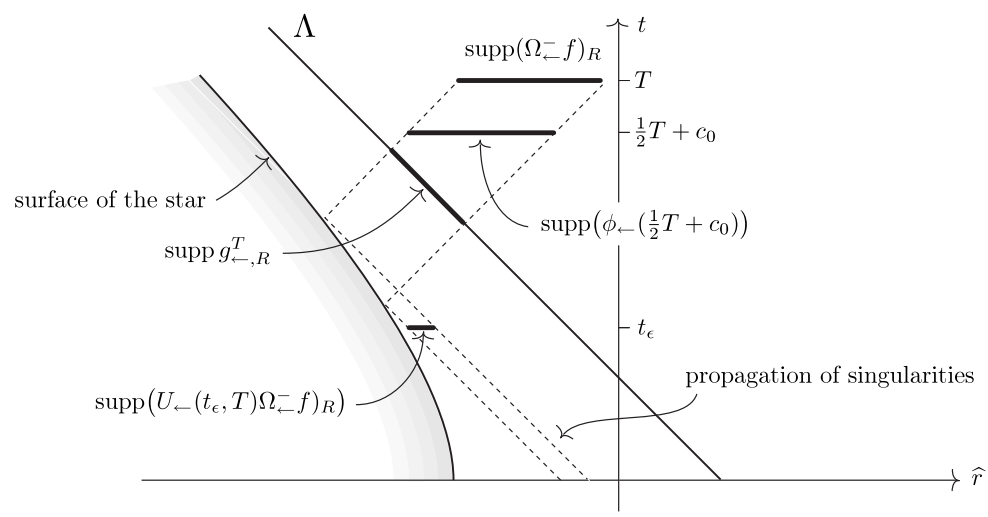

Figure 1. The collapse of the star

effect. However some progress in this direction has been made by Finster, Kamran, Smoller and Yau who obtained an integral representation for the propagator of the wave equation on the Kerr metric (see [22]). We also refer to [7] for scattering results in a superradiant situation.

\section{Notations}

Let $(\mathcal{M}, g)$ be a smooth 4-manifold equipped with a lorentzian metric $g$ with signature $(+,-,-,-)$. We denote by $\nabla_{a}$ the Levi-Civita connection on $(\mathcal{M}, g)$.

Many of our equations will be expressed using the two-component spinor notations and abstract index formalism of R. Penrose and W. Rindler [44].

Abstract indices are denoted by light face latin letters, capital for spinor indices and lower case for tensor indices. Abstract indices are a notational device for keeping track of the nature of objects in the course of calculations, they do not imply any reference to a coordinate basis, all expressions and calculations involving them are perfectly intrinsic. For example, $g_{\mathrm{ab}}$ will refer to the space-time metric as an intrinsic symmetric tensor field of valence $\left[\begin{array}{l}0 \\ 2\end{array}\right]$, i.e. a section of $\mathrm{T}^{*} \mathcal{M} \odot \mathrm{T}^{*} \mathcal{M}$ and $g^{\mathrm{ab}}$ will refer to the inverse metric as an intrinsic symmetric tensor field of valence $\left[\begin{array}{l}2 \\ 0\end{array}\right]$, i.e. a section of $\mathrm{T} \mathcal{M} \odot \mathrm{T} \mathcal{M}$ (where $\odot$ denotes the symmetric tensor product, $\mathrm{T} \mathcal{M}$ the tangent bundle to our space-time manifold $\mathcal{M}$ and $\mathrm{T}^{*} \mathcal{M}$ its cotangent bundle).

Concrete indices defining components in reference to a basis are represented by bold face latin letters. Concrete spinor indices, denoted by bold face capital latin letters, take their values in $\{0,1\}$ while concrete tensor indices, denoted by bold face lower case latin letters, take their values in $\{0,1,2,3\}$. Consider for example a basis of TM, that is a family of four smooth vector fields on $\mathcal{M}: \mathcal{B}=\left\{e_{0}, e_{1}, e_{2}, e_{3}\right\}$ such that at each point $p$ of $\mathcal{M}$ the four vectors $e_{0}(p), e_{1}(p), e_{2}(p), e_{3}(p)$ are linearly independent, and the corresponding dual basis of $\mathrm{T}^{*} \mathcal{M}: \mathcal{B}^{*}=\left\{e^{0}, e^{1}, e^{2}, e^{3}\right\}$ such that $\mathrm{e}^{\boldsymbol{a}}\left(e_{\boldsymbol{b}}\right)=\delta_{\boldsymbol{b}}^{\boldsymbol{a}}$, 
where $\delta_{\boldsymbol{b}}^{\boldsymbol{a}}$ denotes the Kronecker symbol ; $g_{\mathbf{a b}}$ will refer to the components of the metric $g_{\mathrm{ab}}$ in the basis $\mathscr{B}: g_{\mathbf{a b}}=g\left(e_{\boldsymbol{a}}, e_{\boldsymbol{b}}\right)$ and $g^{\mathbf{a b}}$ will denote the components of the inverse metric $g^{\text {ab }}$ in the dual basis $\mathcal{B}^{*}$, i.e. the $4 \times 4$ real symmetric matrices $\left(g_{\mathbf{a b}}\right)$ and $\left(g^{\mathbf{a b}}\right)$ are the inverse of one another. In the abstract index formalism, the basis vectors $e_{\boldsymbol{a}}, \boldsymbol{a}=0,1,2,3$, are denoted $e_{\boldsymbol{a}}{ }^{a}$ or $g_{\boldsymbol{a}}{ }^{a}$. In a coordinate basis, the basis vectors $e_{\boldsymbol{a}}$ are coordinate vector fields and will also be denoted by $\partial_{\boldsymbol{a}}$ or $\partial / \partial x^{\boldsymbol{a}}$; the dual basis covectors $\mathrm{e}^{\boldsymbol{a}}$ are coordinate 1 -forms and will be denoted by $\mathrm{d} x^{\boldsymbol{a}}$.

We adopt Einstein's convention for the same index appearing twice, once up, once down, in the same term. For concrete indices, the sum is taken over all the values of the index. In the case of abstract indices, this signifies the contraction of the index, i.e. $f_{a} V^{a}$ denotes the action of the 1-form $f_{a}$ on the vector field $V^{a}$.

For a manifold $Y$ we denote by $C_{b}^{\infty}(Y)$ the set of all $C^{\infty}$ functions on $Y$, that are bounded together with all their derivatives. We denote by $C_{\infty}(Y)$ the set of all continuous functions tending to zero at infinity.

\section{Acknowledgments}

The author warmly thanks A. Bachelot, J.-F. Bony and J.-P. Nicolas for fruitful discussions. This work was partially supported by the ANR project JC0546063 "Équations hyperboliques dans les espaces-temps de la relativité générale: Diffusion et résonances". 



\section{CHAPTER 2}

\section{STRATEGY OF THE PROOF AND ORGANIZATION OF THE ARTICLE}

\subsection{The analytic problem}

Let us consider a model, where the eternal black hole is described by a static spacetime (although the Kerr-Newman space-time is not even stationary, the problem will be essentially reduced to this kind of situation). Then the problem can be described as follows. Consider a riemannian manifold $\Sigma_{0}$ with one asymptotically euclidean end and a boundary. The boundary will move when $t$ becomes large asymptotically with the speed of light. The manifold at time $t$ is denoted $\Sigma_{t}$. The "limit" manifold $\Sigma$ is a manifold with two ends, one asymptotically euclidean and the other asymptotically hyperbolic (see Figure 1). The problem consists in evaluating the limit

$$
\lim _{T \rightarrow \infty}\left\|\mathbf{1}_{[0, \infty)}\left(\not_{0}\right) U(0, T) f\right\|_{0}
$$

where $U(0, T)$ is the isometric propagator for the Dirac equation on the manifold with moving boundary and suitable boundary conditions. It is worth noting that the underlying scattering theory is not the scattering theory for the problem with moving boundary but the scattering theory on the "limit" manifold. It is largely believed that the result does not depend on the boundary condition. We will show in this paper that it does not depend on the chiral angle in the MIT boundary condition. Note also that the boundary viewed in $\bigcup_{t}\{t\} \times \Sigma_{t}$ is only weakly timelike, a problem that has been rarely considered (but see [4]).

One of the problems for the description of the Hawking effect is to derive a reasonable model for the collapse of the star. We will suppose that the metric outside the collapsing star is always given by the Kerr-Newman metric. Whereas this is a genuine assumption in the rotational case, in the spherically symmetric case Birkhoffs theorem assures that the metric outside the star is the Reissner-Nordström metric. We will suppose that a point on the surface of the star will move along a curve which behaves asymptotically like a timelike geodesic with $L=Q=\widetilde{E}=0$, where $L$ is the angular momentum, $\widetilde{E}$ the rotational energy and $Q$ the Carter constant. The choice 


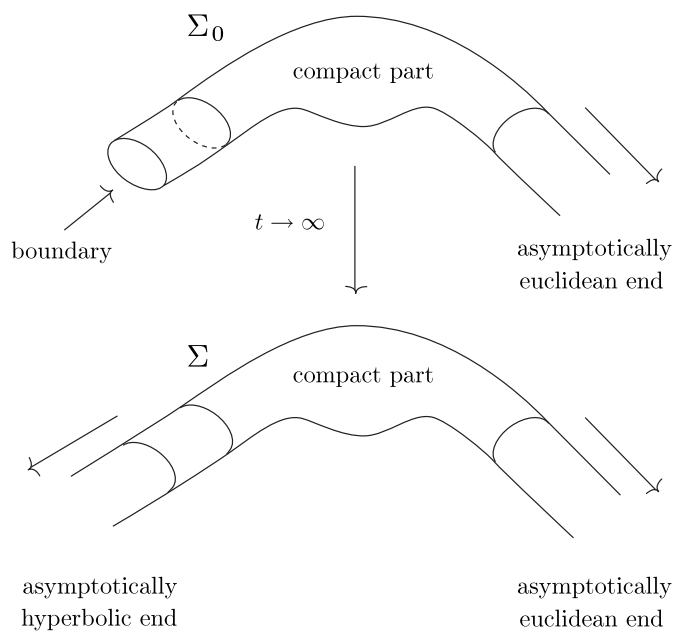

Figure 1 . The manifold $\Sigma_{0}$ at time $t=0$ and the limit manifold $\Sigma$

of geodesics is justified by the fact that the collapse creates the space-time, i.e. angular momenta and rotational energy should be zero with respect to the space-time. We will need an additional asymptotic condition on the collapse. It turns out that there is a natural coordinate system $(t, \widehat{r}, \omega)$ associated to the collapse. In this coordinate system the surface of the star is described by $\widehat{r}=\widehat{z}(t, \theta)$. We need to assume the existence of a constant $C$ such that

$$
|\widehat{z}(t, \theta)+t+C| \longrightarrow 0, \quad t \rightarrow \infty .
$$

It can be checked that this asymptotic condition is fulfilled if we use the above geodesics for some appropriate initial condition. On the one hand we are not able to compute this initial condition explicitly, on the other hand it seems more natural to impose a (symmetric) asymptotic condition than an initial condition. If we would allow in (2.1) a function $C(\theta)$ rather than a constant, the problem would become more difficult. Indeed one of the problems for treating the Hawking radiation in the rotational case is the high frequencies of the solution. In contrast with the spherically symmetric case, the difference between the Dirac operator and an operator with constant coefficients is near the horizon always a differential operator of order one ${ }^{(1)}$. This explains that in the high energy regime we are interested in, the Dirac operator is not close to a constant coefficient operator. Our method to prove (1.3) is to use scattering arguments to reduce the problem to a problem with a constant coefficient operator, for which we can compute the radiation explicitly. If we do not impose a condition of type (2.1), then in all coordinate systems the solution has high frequencies, in the

(1) In the spherically symmetric case we can diagonalize the operator. After diagonalization the difference is just a potential. 
radial as well as in the angular directions. With condition (2.1) these high frequencies only occur in the radial direction. Our asymptotic comparison dynamics will differ from the real dynamics only by derivatives in angular directions and by potentials.

\subsection{Strategy of the proof}

In this section we will give some ideas of the proof of (1.3). We want to reduce the problem to the evaluation of a limit that can be explicitly computed. To do so, we use the asymptotic completeness results obtained in [29] and [15]. There exists a constant coefficient operator $D_{\leftarrow}$ such that the following limits exist:

$$
\begin{aligned}
& W_{\leftarrow}^{ \pm}:=s-\lim _{t \rightarrow \pm \infty} \mathrm{e}^{-i t \not D} \mathrm{e}^{i t \not D_{\leftarrow}} \mathbf{1}_{\mathbb{R}^{\mp}}\left(P_{\leftarrow}^{ \pm}\right), \\
& \Omega_{\leftarrow}^{ \pm}:=s-\lim _{t \rightarrow \pm \infty} \mathrm{e}^{-i t \not D_{\leftarrow}} \mathrm{e}^{i t \not \mathbf{1}_{\mathbb{R} \mp}}\left(P^{ \pm}\right) .
\end{aligned}
$$

Here $P_{\leftarrow}^{ \pm}$is the asymptotic velocity operator associated to the dynamics $\mathrm{e}^{i t \not D_{\leftarrow}}$. Then the R.H.S. of (1.3) equals

$$
\left\|\mathbf{1}_{[0, \infty)}(\not D) \mathbf{1}_{\mathbb{R}^{-}}\left(P^{-}\right) f\right\|^{2}+\left\langle\Omega_{\leftarrow}^{-} f, \mu \mathrm{e}^{\sigma \not D_{\leftarrow}}\left(1+\mu \mathrm{e}^{\sigma \not D_{\leftarrow}}\right)^{-1} \Omega_{\leftarrow}^{-} f\right\rangle .
$$

The aim is to show that the incoming part is

$$
\lim _{T \rightarrow \infty}\left\|\mathbf{1}_{[0, \infty)}\left(D_{\leftarrow, 0}\right) U_{\leftarrow}(0, T) \Omega_{\leftarrow}^{-} f\right\|_{0}^{2}=\left\langle\Omega_{\leftarrow}^{-} f, \mu \mathrm{e}^{\sigma \not \triangleright_{\leftarrow}}\left(1+\mu \mathrm{e}^{\sigma \not D_{\leftarrow}}\right)^{-1} \Omega_{\leftarrow}^{-} f\right\rangle,
$$

where the equality can be shown by explicit calculation. Here $\mathbb{D}_{\leftarrow, t}$ and $U_{\leftarrow}(s, t)$ are the asymptotic operator with boundary condition and the associated propagator. The outgoing part is easy to treat.

As already mentioned, we have to consider the solution in a high frequency regime. Using the Regge-Wheeler variable as a position variable and, say, the Newman-Penrose tetrad used in [29] we find that the modulus of the local velocity

$$
\left[i r_{*}, \not D\right]=h^{2}\left(r_{*}, \omega\right) \Gamma^{1}
$$

is not equal to 1 , whereas the asymptotic dynamics must have constant local velocity. Here $h$ is a continuous function and $\Gamma^{1}$ a constant matrix. Whereas the $\left(r_{*}, \omega\right)$ coordinate system and the tetrad used in [29] were well adapted to the time dependent scattering theory developed in [29], they are no longer well adapted when we consider large times and high frequencies. We are therefore looking for a variable $\widehat{r}$ such that

$$
\{(t, \widehat{r}, \omega) ; \widehat{r} \pm t=\text { Const. }\}
$$

are characteristic surfaces. By a separation of variables Ansatz we find a family of such variables and we choose the one which is well adapted to the collapse of the star in the sense that along an incoming null geodesic with $L=Q=0$ we have

$$
\frac{\partial \widehat{r}}{\partial t}=-1 \text {. }
$$

This variable turns out to be a generalized Bondi-Sachs variable. The null geodesics with $L=Q=0$ are generated by null vector fields $N^{ \pm}$that we choose to be $\ell$ and $n$ in 
the Newman-Penrose tetrad. If we write down the hamiltonian for the Dirac equation with this choice of coordinates and tetrad we find that the local velocity now has modulus 1 everywhere and our initial problem disappears. The new hamiltonian is again denoted $\not D$. Let $D_{\leftarrow}$ be an asymptotic comparison dynamics near the horizon with constant coefficients. Note that (1.3) is of course independent of the choice of the coordinate system and the tetrad, i.e. both sides of (1.3) are independent of these choices. We now proceed as follows:

1) We decouple the problem at infinity from the problem near the horizon by cut-off functions. The problem at infinity is easy to treat.

2) We consider $U(t, T) f$ on a characteristic hypersurface $\Lambda$. The resulting characteristic data is denoted $g^{T}$. We will approximate $\Omega_{\leftarrow}^{-} f$ by a function $\left(\Omega_{\leftarrow}^{-} f\right)_{R}$ with compact support and higher regularity in the angular derivatives. Let $U_{\leftarrow}(s, t)$ be the isometric propagator associated to the asymptotic hamiltonian $D_{\leftarrow}$ with MIT boundary conditions. We also consider $U_{\leftarrow}(t, T)\left(\Omega_{\leftarrow}^{-} f\right)_{R}$ on $\Lambda$. The resulting characteristic data is denoted $g_{\leftarrow, R}^{T}$. The situation for the asymptotic comparison dynamics is shown in Figure 1, Chapter 1.

3) We solve a characteristic Cauchy problem for the Dirac equation with data $g_{\leftarrow, R}^{T}$. The solution at time zero can be written in a region near the boundary as

$$
G\left(g_{\leftarrow, R}^{T}\right)=U\left(0, \frac{1}{2} T+c_{0}\right) \Phi\left(\frac{1}{2} T+c_{0}\right),
$$

where $\Phi$ is the solution of a characteristic Cauchy problem in the whole space (without the star). The solutions of the characteristic problems for the asymptotic hamiltonian are written in a similar way and denoted respectively $G_{\leftarrow}\left(g_{\leftarrow, R}^{T}\right)$ and $\Phi_{\leftarrow}$.

4) Using the asymptotic completeness result we show that $g^{T}-g_{\leftarrow, R}^{T} \rightarrow 0$ when $T, R \rightarrow \infty$. By continuous dependence on the characteristic data we see that

$$
G\left(g^{T}\right)-G\left(g_{\leftarrow, R}^{T}\right) \longrightarrow 0, \quad T, R \rightarrow \infty .
$$

5) We write

$$
\begin{aligned}
G\left(g_{\leftarrow, R}^{T}\right)-G_{\leftarrow}\left(g_{\leftarrow, R}^{T}\right)=U(0, & \left.\frac{1}{2} T+c_{0}\right)\left(\Phi\left(\frac{1}{2} T+c_{0}\right)-\Phi_{\leftarrow}\left(\frac{1}{2} T+c_{0}\right)\right) \\
& +\left(U\left(0, \frac{1}{2} T+c_{0}\right)-U_{\leftarrow}\left(0, \frac{1}{2} T+c_{0}\right)\right) \Phi_{\leftarrow}\left(\frac{1}{2} T+c_{0}\right) .
\end{aligned}
$$

The first term becomes small near the boundary when $T$ becomes large. We then note that for all $\epsilon>0$ there exists $t_{\epsilon}>0$ such that

$$
\left\|\left(U\left(t_{\epsilon}, \frac{1}{2} T+c_{0}\right)-U_{\leftarrow}\left(t_{\epsilon}, \frac{1}{2} T+c_{0}\right)\right) \Phi_{\leftarrow}\left(\frac{1}{2} T+c_{0}\right)\right\|<\epsilon
$$

uniformly in $T$ large. The function $U_{\leftarrow}\left(t_{\epsilon}, \frac{1}{2} T+c_{0}\right) \Phi_{\leftarrow}\left(\frac{1}{2} T+c_{0}\right)$ will be replaced by a geometric optics approximation $F_{t_{\epsilon}}^{T}$ which has the following properties:

$$
\begin{aligned}
& \operatorname{supp} F_{t_{\epsilon}}^{T} \subset\left(-t_{\epsilon}-\left|\theta\left(\mathrm{e}^{-\kappa_{+} T}\right)\right|,-t_{\epsilon}\right), \\
& F_{t_{\epsilon}}^{T} \rightarrow 0, \quad T \rightarrow \infty, \\
& \forall \lambda>0, \quad \mathrm{Op}(\chi(\langle\xi\rangle \leq \lambda\langle q\rangle)) F_{t_{\epsilon}}^{T} \longrightarrow 0, \quad T \rightarrow \infty .
\end{aligned}
$$

Here $\xi$ and $q$ are the dual coordinates to $\widehat{r}, \theta$ respectively. 
6) We show that for $\lambda$ sufficiently large possible singularities of

$$
\mathrm{Op}(\chi(\langle\xi\rangle \geq \lambda\langle q\rangle)) F_{t_{\epsilon}}^{T}
$$

are transported by the group $\mathrm{e}^{-i t_{\epsilon} \not D}$ in such a way that they always stay away from the surface of the star.

7) From the points 1) to 5) follows:

$$
\lim _{T \rightarrow \infty}\left\|\mathbf{1}_{[0, \infty)}\left(\not D_{0}\right) j_{-} U(0, T) f\right\|_{0}^{2}=\lim _{T \rightarrow \infty}\left\|\mathbf{1}_{[0, \infty)}\left(\not_{0}\right) U\left(0, t_{\epsilon}\right) F_{t_{\epsilon}}^{T}\right\|_{0}^{2},
$$

where $j_{-}$is a smooth cut-off which equals 1 near the boundary and 0 at infinity. Let $\phi_{\delta}$ be a cut-off outside the surface of the star at time 0 . If $\phi_{\delta}=1$ sufficiently close to the surface of the star at time 0 we see by the previous point that

$$
\left(1-\phi_{\delta}\right) \mathrm{e}^{-i t_{\epsilon} \not_{D}} F_{t_{\epsilon}}^{T} \longrightarrow 0, \quad T \rightarrow \infty .
$$

Using (2.5) we show that (modulo a small error term)

$$
\left(U\left(0, t_{\epsilon}\right)-\phi_{\delta} \mathrm{e}^{-i t_{\epsilon} \not D}\right) F_{t_{\epsilon}}^{T} \longrightarrow 0, \quad T \rightarrow \infty .
$$

Therefore it remains to consider

$$
\lim _{T \rightarrow \infty}\left\|\mathbf{1}_{[0, \infty)}\left(\not_{0}\right) \phi_{\delta} \mathrm{e}^{-i t_{\epsilon} \not D} F_{t_{\epsilon}}^{T}\right\|_{0} .
$$

$8)$ We show that we can replace $\mathbf{1}_{[0, \infty)}\left(\not D_{0}\right)$ by $\mathbf{1}_{[0, \infty)}(\not D)$. This will essentially allow to commute the energy cut-off and the group. We then show that we can replace the energy cut-off by $\mathbf{1}_{[0, \infty)}\left(\not_{\leftarrow}\right)$. We end up with

$$
\lim _{T \rightarrow \infty}\left\|\mathbf{1}_{[0, \infty)}\left(\not D_{\leftarrow}\right) \mathrm{e}^{-i t_{\epsilon} \not D_{\leftarrow}} F_{t_{\epsilon}}^{T}\right\| .
$$

9) We compute the limit in (2.6) explicitly.

\subsection{Organization of the article}

The paper is organized as follows:

$\triangleright$ In Chapter 3 we present the model of the collapsing star. We first analyze the geodesics in the Kerr-Newman space-time and explain how the Carter constant can be understood in terms of the hamiltonian flow. We construct the variable $\widehat{r}$ and show that

$$
\frac{\partial \widehat{r}}{\partial t}= \pm 1 \text { along null geodesics with } L=Q=0 .
$$

We then show that in the $(t, \widehat{r}, \omega)$ coordinate system we have along incoming timelike geodesics with $L=Q=\widetilde{E}=0$ :

$$
\widehat{r}=-t-\widehat{A}\left(\theta, r_{0}\right) \mathrm{e}^{-2 \kappa_{+} t}+\widehat{B}\left(\theta, r_{0}\right)+\theta\left(\mathrm{e}^{-4 \kappa_{+} t}\right)
$$

with $\widehat{A}\left(\theta, r_{0}\right)>0$. Our assumption will be that a point on the surface behaves asymptotically like $(2.7)$ with $\widehat{B}\left(\theta_{0}, r_{0}\left(\theta_{0}\right)\right)=$ Const. Here $r_{0}\left(\theta_{0}\right)$ is a function defining the surface at time $t=0$. 
$\triangleright$ In Chapter 4 we describe classical Dirac fields. We introduce a new NewmanPenrose tetrad and compute the new expression of the equation. New asymptotic hamiltonians are introduced and classical scattering results are obtained from scattering results in [29] and [15]. The MIT boundary condition is discussed in detail.

$\triangleright$ Dirac quantum fields are discussed in Chapter 5. We first present the second quantization of Dirac fields and then describe the quantization in a globally hyperbolic space-time. The theorem about the Hawking effect is formulated and discussed in Section 5.3.

$\triangleright$ In Chapter 6 we show additional scattering results that we will need later. A minimal velocity estimate slightly stronger than the usual ones is established.

$\triangleright$ In Chapter 7 we solve the characteristic problem for the Dirac equation. We approximate the characteristic surface by smooth spacelike hypersurfaces and recover the solution in the limit. This method is close to that used by Hörmander in [32] for the wave equation.

$\triangleright$ Chapter 8 contains several reductions of the problem. We show that (1.3) implies the theorem about the Hawking effect. We use the axial symmetry to fix the angular momentum. Several technical results are collected.

$\triangleright$ Chapter 9 is devoted to the comparison of the dynamics on the interval $\left[t_{\epsilon}, T\right]$.

$\triangleright$ In Chapter 10 we study the propagation of singularities for the Dirac equation in the Kerr-Newman metric. We show that "outgoing" singularities located in

$$
\left\{(\widehat{r}, \omega, \xi, q) ; \widehat{r} \geq-t_{\epsilon}-C^{-1},|\xi| \geq C|q|\right\}
$$

stay away from the surface of the star for $C$ large.

$\triangleright$ The main theorem is proven in Chapter 11 .

$\triangleright$ Appendix A contains the proof of the existence and uniqueness of solutions of the Dirac equation in the space-time describing the collapsing star.

$\triangleright$ In Appendix B we show that we can compactify the block $I$ of the Kerr-Newman space-time using null geodesics with $L=Q=0$ instead of principal null geodesics. 


\section{CHAPTER 3}

\section{THE MODEL OF THE COLLAPSING STAR}

The purpose of this chapter is to describe the model of the collapsing star. We will suppose that the metric outside the star is given by the Kerr-Newman metric, which is discussed in Section 3.1. Geodesics are discussed in Section 3.1.2. We give a description of the Carter constant in terms of the associated hamiltonian flow. A new position variable is introduced. In Section 3.2 we give the precise asymptotic behavior of the boundary of the star using this new position variable. We require that a point on the surface behaves asymptotically like incoming timelike geodesics with $L=Q=\widetilde{E}=0$, which are studied in Section 3.2.1. The precise assumptions are given in Section 3.2.2.

\subsection{The Kerr-Newman metric}

We give a brief description of the Kerr-Newman metric, which describes an eternal rotating charged black hole. A detailed description can be found e.g. in [48].

3.1.1. Boyer-Lindquist coordinates. - In Boyer-Lindquist coordinates, a KerrNewman black hole is described by a smooth 4-dimensional lorentzian manifold $\mathcal{M}_{\mathrm{BH}}=\mathbb{R}_{t} \times \mathbb{R}_{r} \times S_{\omega}^{2}$, whose space-time metric $g$ and electromagnetic vector potential $\Phi_{a}$ are given by

$$
\left\{\begin{array}{l}
g=\left(1+\frac{Q^{2}-2 M r}{\rho^{2}}\right) \mathrm{d} t^{2}+\frac{2 a \sin ^{2} \theta\left(2 M r-Q^{2}\right)}{\rho^{2}} \mathrm{~d} t \mathrm{~d} \varphi-\frac{\rho^{2}}{\Delta} \mathrm{d} r^{2} \\
\rho^{2}=r^{2}+a^{2} \cos ^{2} \theta, \quad \Delta=r^{2} \mathrm{~d} \theta^{2}-\frac{\sigma^{2}}{\rho^{2}} \sin ^{2} \theta \mathrm{d} \varphi^{2} \\
\sigma^{2}=\left(r^{2}+a^{2}\right) \rho^{2}+\left(2 M r-a^{2}+Q^{2},\right. \\
\Phi_{a} \mathrm{~d} x^{a}=-\frac{Q r}{\rho^{2}}\left(\mathrm{~d} t-a \sin ^{2} \theta \mathrm{d} \varphi\right) .
\end{array}\right.
$$


Here $M$ is the mass of the black hole, $a$ its angular momentum per unit mass and $Q$ the charge of the black hole. If $Q=0, g$ reduces to the Kerr metric, and if $Q=a=0$ we recover the Schwarzschild metric. The expression (3.1) of the Kerr metric has two types of singularities. While the set of points $\left\{\rho^{2}=0\right\}$ (the equatorial ring $\left\{r=0 ; \theta=\frac{1}{2} \pi\right\}$ of the $\{r=0\}$ sphere) is a true curvature singularity, the spheres where $\Delta$ vanishes, called horizons, are mere coordinate singularities. We will consider in this paper subextremal Kerr-Newman space-times, that is we suppose $Q^{2}+a^{2}<M^{2}$. In this case $\Delta$ has two real roots:

$$
r_{ \pm}=M \pm \sqrt{M^{2}-\left(a^{2}+Q^{2}\right)} .
$$

The spheres $\left\{r=r_{-}\right\}$and $\left\{r=r_{+}\right\}$are called event horizons. The two horizons separate $\mathcal{M}_{\mathrm{BH}}$ into three connected components $B_{I}, B_{I I}, B_{I I I}$ called Boyer-Lindquist blocks $\left(r_{+}<r, r_{-}<r<r_{+}, r<r_{-}\right)$. No Boyer-Lindquist block is stationary, that is to say there exists no globally defined timelike Killing vector field on any given block. In particular, block $I$ contains a toroidal region, called the ergosphere, surrounding the horizon,

$$
\mathcal{E}=\left\{(t, r, \theta, \varphi) ; r_{+}<r<M+\sqrt{M^{2}-Q^{2}-a^{2} \cos ^{2} \theta}\right\}
$$

where the vector $\partial / \partial t$ is spacelike.

An important feature of the Kerr-Newman space-time is that it has Petrov type D (see e.g. [42]). This means that the Weyl tensor has two double roots at each point. These roots, referred to as the principal null directions of the Weyl tensor, are given by the two vector fields

$$
V^{ \pm}=\frac{r^{2}+a^{2}}{\Delta} \partial_{t} \pm \partial_{r}+\frac{a}{\Delta} \partial_{\varphi}
$$

Since $V^{+}$and $V^{-}$are twice repeated null directions of the Weyl tensor, by the Goldberg-Sachs theorem (see for example [42, Theorem 5.10.1]) their integral curves define shear-free null geodesic congruences. We shall refer to the integral curves of $V^{+}$ (resp. $V^{-}$) as the outgoing (resp. incoming) principal null geodesics and write from now on PNG for principal null geodesic. The plane determined at each point by the two prinipal null directions is called the principal plane.

We will often use a Regge-Wheeler type coordinate $r_{*}$ in $B_{I}$ instead of $r$ (see [13]), which is given by

$$
r_{*}=r+\frac{1}{2 \kappa_{+}} \ln \left|r-r_{+}\right|-\frac{1}{2 \kappa_{-}} \ln \left|r-r_{-}\right|+R_{0},
$$

where $R_{0}$ is any constant of integration and

$$
\kappa_{ \pm}=\frac{r_{+}-r_{-}}{2\left(r_{ \pm}^{2}+a^{2}\right)}
$$

are the surface gravities at the outer and inner horizons. The variable $r_{*}$ satisfies

$$
\frac{\mathrm{d} r_{*}}{\mathrm{~d} r}=\frac{r^{2}+a^{2}}{\Delta} \text {. }
$$


When $r$ runs from $r_{+}$to $\infty, r_{*}$ runs from $-\infty$ to $\infty$. We put

$$
\Sigma:=\mathbb{R}_{r_{*}} \times S^{2} .
$$

We conclude this section with a useful identity on the coefficients of the metric:

$$
1+\frac{Q^{2}-2 M r}{\rho^{2}}+\frac{a^{2} \sin ^{2} \theta\left(2 M r-Q^{2}\right)^{2}}{\rho^{2} \sigma^{2}}=\frac{\rho^{2} \Delta}{\sigma^{2}} .
$$

3.1.2. Some remarks about geodesics in the Kerr-Newman space-time. It is one of the most remarkable facts about the Kerr-Newman metric that there exist four first integrals for the geodesic equations. If $\gamma$ is a geodesic in the Kerr-Newman space-time, then $p:=\left\langle\gamma^{\prime}, \gamma^{\prime}\right\rangle$ is conserved. The two Killing vector fields $\partial_{t}, \partial_{\varphi}$ give two first integrals, the energy $E:=\left\langle\gamma^{\prime}, \partial_{t}\right\rangle$ and the angular momentum $L:=-\left\langle\gamma^{\prime}, \partial_{\varphi}\right\rangle$. There exists a fourth constant of motion, the so-called Carter constant $\mathcal{K}$ (see [12]). Even if these facts are well known we shall prove them here. The explicit form of the Carter constant in terms of the hamiltonian flow appearing in the proof will be useful in the following. We will also use the Carter constant $Q=\mathcal{K}-(L-a E)^{2}$, which has a somewhat more geometrical meaning, but gives in general more complicated formulas. Let

$$
\boldsymbol{P}:=\left(r^{2}+a^{2}\right) E-a L, \quad \boldsymbol{D}:=L-a E \sin ^{2} \theta .
$$

We will consider the hamiltonian flow of the principal symbol of $\frac{1}{2} \square_{g}$ and then use the fact that a geodesic can be understood as the projection of the hamiltonian flow on $\mathcal{M}_{\mathrm{BH}}$. The d'Alembert operator associated to the Kerr-Newman metric is given by

$$
\begin{aligned}
\square_{g}=\frac{\sigma^{2}}{\rho^{2} \Delta} \partial_{t}^{2}-\frac{2 a\left(Q^{2}-2 M r\right)}{\rho^{2} \Delta} \partial_{\varphi} \partial_{t} & -\frac{\Delta-a^{2} \sin ^{2} \theta}{\rho^{2} \Delta \sin ^{2} \theta} \partial_{\varphi}^{2} \\
& -\frac{1}{\rho^{2}} \partial_{r} \Delta \partial_{r}-\frac{1}{\rho^{2}} \frac{1}{\sin \theta} \partial_{\theta} \sin \theta \partial_{\theta} .
\end{aligned}
$$

The principal symbol of $\frac{1}{2} \square_{g}$ is

$$
P:=\frac{1}{2 \rho^{2}}\left(\frac{\sigma^{2}}{\Delta} \tau^{2}-\frac{2 a\left(Q^{2}-2 M r\right)}{\Delta} q_{\varphi} \tau-\frac{\Delta-a^{2} \sin ^{2} \theta}{\Delta \sin ^{2} \theta} q_{\varphi}^{2}-\Delta|\xi|^{2}-q_{\theta}^{2}\right) .
$$

Let

$$
\mathscr{C}_{p}:=\left\{\left(t, r, \theta, \varphi ; \tau, \xi, q_{\theta}, q_{\varphi}\right) ; P\left(t, r, \theta, \varphi ; \tau, \xi, q_{\theta}, q_{\varphi}\right)=\frac{1}{2} p\right\}
$$

Here $\left(\tau, \xi, q_{\theta}, q_{\varphi}\right)$ is dual to $(t, r, \theta, \varphi)$. We have the following:

THEOREM 3.1. - (i) Let $x_{0}=\left(t_{0}, r_{0}, \varphi_{0}, \theta_{0}, \tau_{0}, \xi_{0}, q_{\theta_{0}}, q_{\varphi_{0}}\right) \in \mathscr{C}_{p}$ and

$$
x(s)=\left(t(s), r(s), \theta(s), \varphi(s) ; \tau(s), \xi(s), q_{\theta}(s), q_{\varphi}(s)\right)
$$


be the associated hamiltonian flow line. Then we have the following constants of motion

$$
\left\{\begin{array}{l}
p=2 P, \quad E=\tau, \quad L=-q_{\varphi}, \\
\mathcal{K}=q_{\theta}^{2}+\frac{\boldsymbol{D}^{2}}{\sin ^{2} \theta}+p a^{2} \cos ^{2} \theta=\frac{\boldsymbol{P}^{2}}{\Delta}-\Delta|\xi|^{2}-p r^{2},
\end{array}\right.
$$

where $\boldsymbol{D}, \boldsymbol{P}$ are defined in (3.9).

It follows:

Corollary 3.1. - Let $\gamma$ with $\gamma^{\prime}=t^{\prime} \partial_{t}+r^{\prime} \partial_{r}+\theta^{\prime} \partial_{\theta}+\varphi^{\prime} \partial_{\varphi}$ be a geodesic in the Kerr-Newman space-time. Then there exists a constant $\mathcal{K}=\mathcal{K}_{\gamma}$ such that

$$
\begin{gathered}
\rho^{4}\left(r^{\prime}\right)^{2}=R(r)=\Delta\left(-p r^{2}-\mathcal{K}\right)+\boldsymbol{P}^{2}, \\
\rho^{4}\left(\theta^{\prime}\right)^{2}=\Theta(\theta)=\mathcal{K}-p a^{2} \cos ^{2} \theta-\frac{\boldsymbol{D}^{2}}{\sin ^{2} \theta}, \\
\rho^{2} \varphi^{\prime}=\frac{\boldsymbol{D}}{\sin ^{2} \theta}+\frac{a \boldsymbol{P}}{\Delta}, \\
\rho^{2} t^{\prime}=a \boldsymbol{D}+\left(r^{2}+a^{2}\right) \frac{\boldsymbol{P}}{\Delta} .
\end{gathered}
$$

REMARK 3.1. - Theorem 3.1 explains the link between the Carter constant $\mathcal{K}$ and the separability of the wave equation. Looking for $f$ in the form

$$
f(t, r, \theta, \varphi)=\mathrm{e}^{i \omega t} \mathrm{e}^{i n \varphi} f_{r}(r) f_{\theta}(\theta)
$$

we find

$$
\begin{aligned}
& \square_{g} f=0 \\
& \qquad\left(-\frac{\left(r^{2}+a^{2}\right)^{2}}{\Delta} \omega^{2}+\frac{2 a\left(Q^{2}-2 M r\right)}{\Delta} n \omega+\frac{r^{2}+a^{2}}{\Delta} D_{r_{*}}\left(r^{2}+a^{2}\right) D_{r_{*}}-\frac{a^{2} n^{2}}{\Delta}\right) f \\
& \quad+\left(a^{2} \sin ^{2} \theta \omega^{2}+\frac{n^{2}}{\sin ^{2} \theta}+\frac{1}{\sin \theta} D_{\theta} \sin \theta D_{\theta}\right) f=0 .
\end{aligned}
$$

For fixed $\omega$

$$
P_{S^{2}}^{\omega}:=a^{2} \sin ^{2} \theta \omega^{2}+\frac{D_{\varphi}^{2}}{\sin ^{2} \theta}+\frac{1}{\sin \theta} D_{\theta} \sin \theta D_{\theta}
$$

is a positive elliptic operator on the sphere with eigenfunctions of the form $\mathrm{e}^{i n \varphi} f_{\theta}(\theta)$. This gives the separability of the equation. The Carter constant is the analogue of the eigenvalue of $P_{S^{2}}^{\omega}$ in classical mechanics.

Proof of Theorem 3.1. - The hamiltonian equations are:

$$
\begin{aligned}
& \dot{t}=\left(\frac{\sigma^{2}}{\rho^{2} \Delta} \tau-a \frac{Q^{2}-2 M r}{\rho^{2} \Delta} q_{\varphi}\right), \\
& \dot{\tau}=0,
\end{aligned}
$$




$$
\begin{aligned}
\dot{r} & =-\frac{\Delta}{\rho^{2}} \xi \\
\dot{\xi} & =-\partial_{r} P \\
\dot{\theta} & =-\frac{q_{\theta}}{\rho^{2}}, \\
\dot{q}_{\theta} & =\frac{a^{2} \sin \theta \cos \theta}{\rho^{2}} \tau^{2}+\frac{1}{2 \rho^{2}} \partial_{\theta} \frac{q_{\varphi}^{2}}{\sin ^{2} \theta}-\frac{a^{2} \cos \theta \sin \theta}{\rho^{2}} p, \\
\dot{\varphi} & =\left(\frac{a\left(2 M r-Q^{2}\right)}{\rho^{2} \Delta} \tau-\frac{\Delta-a^{2} \sin ^{2} \theta}{\rho^{2} \Delta \sin ^{2} \theta} q_{\varphi}\right), \\
\dot{q}_{\varphi} & =0,
\end{aligned}
$$

where we have used that $\left(t, r, \theta, \varphi, \tau, \xi, q_{\theta}, q_{\varphi}\right)$ stays in $\mathscr{C}_{p}$. The first two constants of motion follow from (3.18), (3.24). We multiply (3.22) with $q_{\theta}$ given by (3.21) and obtain

$$
\begin{aligned}
\dot{q}_{\theta} q_{\theta} & =-a^{2} \sin \theta \cos \theta \tau^{2} \dot{\theta}-\frac{1}{2} \frac{\mathrm{d}}{\mathrm{d} t} \frac{q_{\varphi}^{2}}{\sin ^{2} \theta}+a^{2} \cos \theta \sin \theta \dot{\theta} p \\
& =-\frac{1}{2} \frac{\mathrm{d}}{\mathrm{d} t}\left(a^{2} \sin ^{2} \theta \tau^{2}+\frac{q_{\varphi}^{2}}{\sin ^{2} \theta}+a^{2} \cos ^{2} \theta p\right) \\
& \Longrightarrow q_{\theta}^{2}+a^{2} \sin ^{2} \theta \tau^{2}+\frac{q_{\varphi}^{2}}{\sin ^{2} \theta}+a^{2} \cos ^{2} \theta p=\widehat{\mathcal{K}}=\text { Const. }
\end{aligned}
$$

To obtain the second expression for $\mathcal{K}$ we use the fact that the flow stays in $\mathscr{C}_{p}$.

The case $L=0$ is of particular interest. Let $\gamma$ be a null geodesic with energy $E>0$, Carter constant $\mathcal{K}$, angular momentum $L=0$ and given signs of $r_{0}^{\prime}, \theta_{0}^{\prime}$. We can associate a hamiltonian flow line using (3.12) to define the initial data $\tau_{0}, \xi_{0}, q_{\theta_{0}}, q_{\varphi_{0}}$ given $t_{0}, r_{0}, \theta_{0}, \varphi_{0}$. The signs of $q_{\theta_{0}}$ and $\xi_{0}$ are fixed by $\operatorname{sign} q_{\theta_{0}}=-\operatorname{sign} \theta_{0}^{\prime}$ and $\operatorname{sign} \xi_{0}=-\operatorname{sign} r_{0}^{\prime}$. From (3.12) we infer conditions under which $\xi, q_{\theta}$ do not change their signs:

$$
\begin{gathered}
\mathcal{K}<\min _{r \in\left(r_{+}, \infty\right)} \frac{\left(r^{2}+a^{2}\right)^{2} E^{2}}{\Delta} \Longrightarrow \xi \text { does not change its sign, } \\
Q \geq 0 \Longrightarrow q_{\theta} \text { does not change its sign. }
\end{gathered}
$$

Note that in the case $Q=0 \gamma$ is either in the equatorial plane or it does not cross it. Under the above conditions $\xi$ (resp. $q_{\theta}$ ) can be understood as a function of $r$ (resp. $\theta$ ) alone. In this case let $k_{\mathcal{K}, E}$ and $\ell_{\mathcal{K}, E}$ such that

$$
\frac{\mathrm{d} k_{\mathcal{K}, E}(r)}{\mathrm{d} r}=\frac{\xi(r)}{E}, \quad \ell_{\mathcal{K}, E}^{\prime}=\frac{q_{\theta}(\theta)}{E}, \quad \widehat{r}_{\mathcal{K}, E}:=k_{\mathcal{K}, E}(r)+\ell_{\mathcal{K}, E}(\theta) .
$$

It is easy to check that $\left(t, \widehat{r}_{\mathcal{K}, E}, \omega\right)$ is a coordinate system on block $I$. We note that by (3.16) we have $\partial_{s} t=E \sigma^{2} / \Delta>0$, thus $r, \theta, \varphi$ can be understood as functions of $t$ along $\gamma$. 
LEMMA 3.1. - If $t$ is the Boyer-Lindquist time, we have

$$
\frac{\partial \widehat{r}_{\mathcal{K}, E}}{\partial t}=-1 \quad \text { along } \gamma
$$

Proof. - This is an explicit calculation using equations (3.13)-(3.16), (3.17)-(3.24):

$$
\begin{aligned}
\frac{\partial \widehat{r}_{\mathcal{K}, E}}{\partial t} & =\frac{\partial \widehat{r}_{\mathcal{K}, E}}{\partial r} \cdot \frac{\partial r}{\partial s} \cdot \frac{\partial s}{\partial t}+\frac{\partial \widehat{r}_{\mathcal{K}, E}}{\partial \theta} \cdot \frac{\partial \theta}{\partial s} \cdot \frac{\partial s}{\partial t} \\
& =-\left(|\xi|^{2} \Delta+\left|q_{\theta}\right|^{2}\right) \frac{1}{E}\left(a \boldsymbol{D}+\left(r^{2}+a^{2}\right) \frac{\boldsymbol{P}}{\Delta}\right)^{-1} \\
& =-\frac{1}{E}\left(\frac{\boldsymbol{P}^{2}}{\Delta}-\frac{\boldsymbol{D}^{2}}{\sin ^{2} \theta}\right)\left(a \boldsymbol{D}+\left(r^{2}+a^{2}\right) \frac{\boldsymbol{P}}{\Delta}\right)^{-1}=-1 .
\end{aligned}
$$

We will suppose from now on $r_{0}^{\prime}<0$, i.e. our construction is based on incoming null geodesics.

REMARK 3.2. - Using the axial symmetry of the Kerr-Newman space-time we can for many studies of field equations in this background fix the angular momentum $\partial_{\varphi}=i n$ in the expression of the operator. The principal symbol of the new operator is the principal symbol of the old one with $q_{\varphi}=0$. This explains the importance of Lemma 3.1.

We will often use the $r_{*}$ variable and its dual variable $\xi^{*}$. In this case we have to replace $\xi(r)$ by $\left(\left(r^{2}+a^{2}\right) / \Delta\right) \xi^{*}\left(r_{*}\right)$. The function $k_{\mathcal{K}, E}$ is then a function of $r_{*}$ satisfying

$$
k_{\mathcal{K}, E}^{\prime}\left(r_{*}\right)=\frac{\xi^{*}}{E}
$$

where the prime denotes derivation with respect to $r_{*}$. Using the explicit form of the Carter constant in Theorem 3.1 we find

$$
\begin{aligned}
\left(k_{\mathcal{K}, E}^{\prime}\right)^{2} & =1-\frac{\Delta \mathcal{K}}{\left(r^{2}+a^{2}\right)^{2} E^{2}}, \\
\left(\ell_{\mathcal{K}, E}^{\prime}\right)^{2} & =\frac{\mathcal{K}}{E^{2}}-a^{2} \sin ^{2} \theta .
\end{aligned}
$$

In particular we have

$$
\left(k_{\mathcal{K}, E}^{\prime}\right)^{2} \frac{\left(r^{2}+a^{2}\right)^{2}}{\sigma^{2}}+\left(\ell_{\mathcal{K}, E}^{\prime}\right)^{2} \frac{\Delta}{\sigma^{2}}=1
$$

We will often consider the case $Q=0$ and write in this case simply $k, \ell$ instead of $k_{a^{2} E^{2}, E}$ and $\ell_{a^{2} E^{2}, E}$. 
REMARK 3.3. - The incoming null geodesics with Carter constant $\mathcal{K}$, angular momentum $L=0$, energy $E>0$ and given sign of $\theta_{0}^{\prime}$ are the integral curves of the following vector fields

$$
\begin{aligned}
M_{\mathcal{K}, E}^{a}=\frac{E}{\rho^{2}}\left(\frac{\sigma^{2}}{\Delta} \partial_{t}-\left(r^{2}+a^{2}\right) k_{\mathcal{K}, E}^{\prime}\left(r_{*}(r)\right) \partial_{r}\right. & \\
& \left.-\ell_{\mathcal{K}, E}^{\prime}(\theta) \partial_{\theta}+\frac{a\left(2 M r-Q^{2}\right)}{\Delta} \partial_{\varphi}\right) .
\end{aligned}
$$

Let us put

$$
\begin{aligned}
V_{1}^{a} \partial_{a} & =\sqrt{\sigma^{2} / \rho^{2} \Delta}\left(\partial_{t}+\frac{a\left(2 M r-Q^{2}\right)}{\sigma^{2}} \partial_{\varphi}\right), \\
V_{2}^{a} \partial_{a} & =\sqrt{\Delta / \rho^{2} \sigma^{2}}\left(\left(r^{2}+a^{2}\right) k_{\mathcal{K}, E}^{\prime}\left(r_{*}(r)\right) \partial_{r}+\ell_{\mathcal{K}, E}^{\prime}(\theta) \partial_{\theta}\right), \\
W_{1}^{a} \partial_{a} & =\frac{\sqrt{\rho^{2} / \sigma^{2}}}{\sin \theta} \partial_{\varphi}, \\
W_{2}^{a} \partial_{a} & =\frac{r^{2}+a^{2}}{\sqrt{\rho^{2} \sigma^{2}}}\left(\frac{\Delta}{r^{2}+a^{2}} \ell_{\mathcal{K}, E}^{\prime} \partial_{r}-k_{\mathcal{K}, E}^{\prime} \partial_{\theta}\right) .
\end{aligned}
$$

Note that

$$
\begin{aligned}
& V_{1}^{a} V_{1 a}=1, \quad V_{2}^{a} V_{2 a}=W_{1}^{a} W_{1 a}=W_{2}^{a} W_{2 a}=-1, \\
& L^{a} N_{a}=0 \quad \forall N^{a}, L^{a} \in\left\{V_{1}^{a}, V_{2}^{a}, W_{1}^{a}, W_{2}^{a}\right\}, L^{a} \neq N^{a} .
\end{aligned}
$$

Clearly the considered null geodesics lie in $\Pi=\operatorname{span}\left\{V_{1}^{a}, V_{2}^{a}\right\}$. Using the Frobenius theorem (see e.g. [42, Theorem 1.7.4]) we see that, in contrast to the PNG case, in our case the distribution of planes $\Pi^{\perp}=\operatorname{span}\left\{W_{1}^{a}, W_{2}^{a}\right\}$ is integrable.

Corollary 3.2. - For given Carter constant $\mathcal{K}$, energy $E>0$ and sign of $\theta_{0}^{\prime}$ the following surfaces are characteristic:

$$
\mathscr{C}_{\mathcal{K}, E}^{c, \pm}=\left\{\left(t, r_{*}, \theta, \varphi\right) ; \pm t=\widehat{r}_{\mathcal{K}, E}\left(r_{*}, \theta\right)+c\right\} .
$$

Proof. - By Lemma 3.1 the incoming null geodesic $\gamma$ with Carter constant $\mathcal{K}$, energy $E$, angular momentum $L=0$ and the correct sign of $\theta_{0}^{\prime}$ lies entirely in $\mathscr{C}_{\mathcal{K}, E}^{c,-}$ if the starting point lies in it. The geodesic $\gamma(-s)$ lies in $\mathscr{C}_{\mathcal{K}, E}^{c,+}$.

REMARK 3.4. - (i) The variable $\widehat{r}_{\mathcal{K}, E}$ is a Bondi-Sachs type coordinate. This coordinate system is discussed in some detail in [23]. As in [23] we will call the null geodesics with $L=Q=0$ simple null geodesics (SNG's).

(ii) A natural way of finding the variable $\widehat{r}_{\mathcal{K}, E}$ is to start with Corollary 3.2. Look for functions $k_{\mathcal{K}, E}\left(r_{*}\right)$ and $\ell_{\mathcal{K}, E}(\theta)$ such that $\mathscr{C}_{\mathcal{K}, E}^{c, \pm}=\left\{ \pm t=k_{\mathcal{K}, E}\left(r_{*}\right)+\ell_{\mathcal{K}, E}(\theta)\right\}$ is characteristic. The condition that the normal is null is equivalent to (3.30). The curve generated by the normal lies entirely in $\mathscr{C}_{\mathcal{K}, E}^{c, \pm}$. 
REMARK 3.5. - From the explicit form of the Carter constant in Theorem 3.1 follows:

$$
q_{\theta}^{2}+\left(p-E^{2}\right) a^{2} \cos ^{2} \theta+\frac{q_{\varphi}^{2}}{\sin ^{2} \theta}=Q
$$

This is the equation of the $\theta$ motion and it is interpreted as conservation of the mechanical energy with $V(\theta)=\left(p-E^{2}\right) a^{2} \cos ^{2} \theta+q_{\varphi}^{2} / \sin ^{2} \theta$ as potential energy and $q_{\theta}^{2}$ in the role of kinetic energy. The quantity $\widetilde{E}=\left(E^{2}-p\right) a^{2}$ is usually called the rotational energy.

\subsection{The model of the collapsing star}

Let $\phi_{0}$ be the surface of the star at time $t=0$. We suppose that elements $x_{0} \in \phi_{0}$ will move along curves which behave asymptotically like certain incoming timelike geodesics $\gamma_{p}$. All these geodesics should have the same energy $E$, angular momentum $L$, Carter constant $\mathcal{K}$ (resp. $\left.Q=\mathcal{K}-(L-a E)^{2}\right)$ and "mass" $p:=\left\langle\gamma_{p}^{\prime}, \gamma_{p}^{\prime}\right\rangle$. We will suppose:

(A) The angular momentum $L$ vanishes: $L=0$.

(B) The rotational energy vanishes: $\widetilde{E}=a^{2}\left(E^{2}-p\right)=0$.

(C) The total angular momentum about the axis of symmetry vanishes: $Q=0$.

Conditions $(\mathrm{A})-(\mathrm{C})$ are imposed by the fact that the collapse itself creates the space-time, thus momenta and rotational energy should be zero with respect to the space-time.

3.2.1. Timelike geodesics with $L=Q=\widetilde{E}=0$. - We study the above family of geodesics. The starting point of the geodesic is denoted $\left(0, r_{0}, \theta_{0}, \varphi_{0}\right)$. Given a point in the space-time, the conditions (A)-(C) define a unique cotangent vector provided you add the condition that the corresponding tangent vector is incoming. The choice of $p$ is irrelevant because it just corresponds to a normalization of the proper time.

Lemma 3.2. - Along the geodesic $\gamma_{p}$ we have:

$$
\begin{aligned}
\frac{\partial \theta}{\partial t} & =0, \\
\frac{\partial \varphi}{\partial t} & =\frac{a\left(2 M r-Q^{2}\right)}{\sigma^{2}},
\end{aligned}
$$

where $t$ is the Boyer-Lindquist time.

Proof. - Equation (3.33) follows directly from (3.14). We have

$$
\begin{aligned}
\frac{\partial \varphi}{\partial t} & =\frac{\partial \varphi}{\partial s} \frac{\partial s}{\partial t}=\frac{1}{\rho^{2}}\left(-a E+\frac{a\left(r^{2}+a^{2}\right) E}{\Delta}\right) \rho^{2}\left(-a^{2} E \sin ^{2} \theta+\frac{\left(r^{2}+a^{2}\right)^{2}}{\Delta} E\right)^{-1} \\
& =\frac{\Delta a}{\sigma^{2} \Delta}\left(r^{2}+a^{2}-\Delta\right)=\frac{a\left(2 M r-Q^{2}\right)}{\sigma^{2}} .
\end{aligned}
$$


The function $\partial \varphi / \partial t=a\left(2 M r-Q^{2}\right) / \sigma^{2}$ is usually called the local angular velocity of the space-time. Our next aim is to adapt our coordinate system to the collapse of the star. The most natural way of doing this is to choose an incoming null geodesic $\gamma$ with $L=Q=0$ and then use the Bondi-Sachs type coordinate as in the previous section. In addition we want that $k\left(r_{*}\right)$ behaves like $r_{*}$ when $r_{*} \rightarrow-\infty$. We therefore put

$$
\begin{aligned}
k\left(r_{*}\right) & =r_{*}+\int_{-\infty}^{r_{*}}\left(\sqrt{1-\frac{a^{2} \Delta(s)}{\left(r(s)^{2}+a^{2}\right)^{2}}}-1\right) \mathrm{d} s, \\
\ell(\theta) & =a \sin \theta .
\end{aligned}
$$

The choice of the sign of $\ell^{\prime}$ is not important, the opposite sign would have been possible. Recall that $\cos \theta$ does not change its sign along a null geodesic with $L=Q=0$. We fix the notation for the null vector fields generating $\gamma$ and the corresponding outgoing vector field (see Remark 3.3):

$$
\begin{aligned}
N^{ \pm, a} \partial_{a}=\frac{E \sigma^{2}}{\rho^{2} \Delta}\left(\partial_{t} \pm \frac{\left(r^{2}+a^{2}\right)^{2}}{\sigma^{2}} k^{\prime}\left(r_{*}\right) \partial_{r_{*}}\right. & \\
& \left. \pm \frac{\Delta}{\sigma^{2}} a \cos \theta \partial_{\theta}+\frac{a\left(2 M r-Q^{2}\right)}{\sigma^{2}} \partial_{\varphi}\right) .
\end{aligned}
$$

These vector fields will be important for the construction of the Newman-Penrose tetrad. We put

$$
\widehat{r}=k\left(r_{*}\right)+\ell(\theta)
$$

and by Lemma 3.1 we have

$$
\frac{\partial \widehat{r}}{\partial t}=-1 \quad \text { along } \gamma
$$

Note that in the $(t, \widehat{r}, \omega)$ coordinate system the metric is given by

$$
\begin{aligned}
g=\left(1+\frac{Q^{2}-2 M r}{\rho^{2}}\right) \mathrm{d} t^{2}+\frac{2 a \sin ^{2} \theta\left(2 M r-Q^{2}\right)}{\rho^{2}} \mathrm{~d} t \mathrm{~d} \varphi \\
\quad-\frac{\rho^{2} \Delta}{\left(r^{2}+a^{2}\right)^{2}} \frac{\left(\mathrm{d} \widehat{r}-\ell^{\prime}(\theta) \mathrm{d} \theta\right)^{2}}{k^{\prime}\left(r_{*}\right)^{2}}-\rho^{2} \mathrm{~d} \theta^{2}-\frac{\sigma^{2}}{\rho^{2}} \sin ^{2} \theta \mathrm{d} \varphi^{2} .
\end{aligned}
$$

In order to describe the model of the collapsing star we have to evaluate $\partial \widehat{r} / \partial t$ along $\gamma_{p}$. We start by studying $r(t, \theta)$. Recall that $\theta(t)=\theta_{0}=$ Const. along $\gamma_{p}$ and that $\kappa_{+}=\frac{1}{2}\left(r_{+}-r_{-}\right) /\left(r_{+}^{2}+a^{2}\right)$ is the surface gravity of the outer horizon. In what follows a dot will denote derivation in $t$.

LEMMA 3.3. - There exist smooth functions $\widehat{C}_{1}\left(\theta, r_{0}\right)$ and $\widehat{C}_{2}\left(\theta, r_{0}\right)$ such that along $\gamma_{p}$ we have uniformly in $\theta, r_{0} \in\left[r_{1}, r_{2}\right] \subset\left(r_{+}, \infty\right)$ :

$$
\frac{1}{2 \kappa_{+}} \ln \left|r-r_{+}\right|=-t-\widehat{C}_{1}\left(\theta, r_{0}\right) \mathrm{e}^{-2 \kappa_{+} t}+\widehat{C}_{2}\left(\theta, r_{0}\right)+\theta\left(\mathrm{e}^{-4 \kappa_{+} t}\right), \quad t \rightarrow \infty .
$$


Proof. - Note that $\left(r^{2}+a^{2}\right)>\Delta$ on $\left[r_{+}, \infty\right)$. Therefore $\partial r / \partial t$ cannot change its sign. As $\gamma_{p}$ is incoming, the minus sign has to be chosen. From (3.13), (3.16) we find with $p=E^{2}$ :

$$
\frac{\partial r}{\partial t}=-\left(\left(r^{2}+a^{2}\right)\left(2 M r-Q^{2}\right)\right)^{\frac{1}{2}} \frac{\Delta}{\sigma^{2}}
$$

We can consider $\theta$ as a parameter. Equation (3.41) gives

$$
\begin{aligned}
t & =-\int_{r_{0}}^{r} \frac{\sigma^{2}}{\left(\left(s^{2}+a^{2}\right)\left(2 M s-Q^{2}\right)\right)^{\frac{1}{2}} \Delta} \mathrm{d} s \\
& =-\frac{1}{2 \kappa_{+}} \ln \left|r-r_{+}\right|+\widehat{C}_{2}-\int_{r_{+}}^{r} P(s) \mathrm{d} s
\end{aligned}
$$

with

$$
\begin{aligned}
P(r) & :=\left(\frac{\sigma^{2}}{\left(\left(r^{2}+a^{2}\right)\left(2 M r-Q^{2}\right)\right)^{\frac{1}{2}}\left(r-r_{-}\right)}-\frac{1}{2 \kappa_{+}}\right) \frac{1}{r-r_{+}} \\
& =\left(\frac{P_{1}(r)}{P_{2}(r)}-\frac{1}{2 \kappa_{+}}\right) \frac{1}{r-r_{+}}=\frac{P_{1}^{2}(r)-\frac{1}{4 \kappa_{+}^{2}} P_{2}^{2}(r)}{P_{2}(r)\left(P_{1}(r)+\frac{1}{2 \kappa_{+}} P_{2}(r)\right)} \frac{1}{r-r_{+}}, \\
\widehat{C}_{2} & :=\frac{1}{2 \kappa_{+}} \ln \left|r_{0}-r_{+}\right|+\int_{r_{+}}^{r_{0}} P(s) \mathrm{d} s .
\end{aligned}
$$

Note that $P_{1}^{2}\left(r_{+}\right)-\left(1 / 4 \kappa_{+}^{2}\right) P_{2}^{2}\left(r_{+}\right)=0$. As $P_{1}^{2}(r)-\left(1 / 4 \kappa_{+}^{2}\right) P_{2}^{2}(r)$ is a polynomial we infer that $P(r)$ is smooth at $r_{+}$. Let

$$
F(r):=\frac{1}{r-r_{+}} \int_{r_{+}}^{r} P(s) \mathrm{d} s .
$$

Clearly $F$ is smooth and $\lim _{r \rightarrow r_{+}} F(r)=P\left(r_{+}\right)=: F\left(r_{+}\right)$. From (3.42) we infer:

$$
\begin{aligned}
& r-r_{+}=\mathrm{e}^{-2 \kappa_{+} t} \mathrm{e}^{2 \kappa_{+}\left(\widehat{C}_{2}-\left(r-r_{+}\right) F(r)\right),} \\
& r-r_{+}=\mathrm{e}^{-2 \kappa_{+} t} \mathrm{e}^{2 \kappa_{+} \widehat{C}_{2}} \mathrm{e}^{f(t)}
\end{aligned}
$$

with $f(t)=\theta\left(\mathrm{e}^{-2 \kappa_{+} t}\right)$. Putting (3.44) into (3.43) we obtain

$$
r-r_{+}=\mathrm{e}^{-2 \kappa_{+} t} \mathrm{e}^{2 \kappa_{+}\left(\widehat{C}_{2}-\mathrm{e}^{-2 \kappa}+{ }^{t} \mathrm{e}^{2 \kappa_{+}} \widehat{C}_{2} \mathrm{e}^{f(t)} F(r)\right)}
$$

so that

$$
\begin{aligned}
\frac{1}{2 \kappa_{+}} \ln \left|r-r_{+}\right|=-t+\widehat{C}_{2}- & \mathrm{e}^{-2 \kappa_{+} t} \mathrm{e}^{2 \kappa_{+} \widehat{C}_{2}} \mathrm{e}^{f(t)} F(r) \\
=-t+\widehat{C}_{2}- & \mathrm{e}^{-2 \kappa_{+} t} \mathrm{e}^{2 \kappa_{+} \widehat{C}_{2}} F\left(r_{+}\right) \\
& \quad+\mathrm{e}^{2 \kappa_{+}\left(\widehat{C}_{2}-t\right)}\left(F\left(r_{+}\right)-\mathrm{e}^{f(t)} F(r)\right)
\end{aligned}
$$

and it remains to show

$$
F\left(r_{+}\right)-\mathrm{e}^{f(t)} F(r)=\varnothing\left(\mathrm{e}^{-2 \kappa_{+} t}\right) .
$$


We write

$$
F\left(r_{+}\right)-\mathrm{e}^{f(t)} F(r)=F\left(r_{+}\right)-F(r)+\left(1-\mathrm{e}^{f(t)}\right) F(r) .
$$

Noting that $\mathrm{e}^{f(t)}=1+\theta\left(\mathrm{e}^{-2 \kappa_{+} t}\right)$ we obtain the required estimate for the second term in (3.46). To estimate the first term we write

$$
F\left(r_{+}\right)-F(r)=\int_{r_{+}}^{r} \frac{P\left(r_{+}\right)-P(s)}{r-r_{+}} \mathrm{d} s
$$

As $\left|P(s)-P\left(r_{+}\right)\right| \lesssim\left|s-r_{+}\right|$we have

$$
\left|F\left(r_{+}\right)-F(r)\right| \lesssim \int_{r_{+}}^{r}\left|\frac{s-r_{+}}{r-r_{+}}\right| \mathrm{d} s \lesssim\left|r-r_{+}\right|=\Theta\left(\mathrm{e}^{-2 \kappa_{+} t}\right)
$$

Equality (3.45) follows. From the explicit form of the equations it is clear that everything is uniform in $\theta, r_{0} \in\left[r_{1}, r_{2}\right]$.

LEMmA 3.4. - (i) There exist smooth functions $A\left(\theta, r_{0}\right), B\left(\theta, r_{0}\right)$ such that along $\gamma_{p}$ we have uniformly in $\theta, r_{0} \in\left[r_{1}, r_{2}\right] \subset\left(r_{+}, \infty\right)$ :

$$
r_{*}=-t-A\left(\theta, r_{0}\right) \mathrm{e}^{-2 \kappa_{+} t}+B\left(\theta, r_{0}\right)+\theta\left(\mathrm{e}^{-4 \kappa_{+} t}\right), \quad t \rightarrow \infty .
$$

(ii) There exist smooth functions $\widehat{A}\left(\theta, r_{0}\right)>0, \widehat{B}\left(\theta, r_{0}\right)$ such that along $\gamma_{p}$ we have uniformly in $\theta, r_{0} \in\left[r_{1}, r_{2}\right] \subset\left(r_{+}, \infty\right)$ :

$$
\widehat{r}=-t-\widehat{A}\left(\theta, r_{0}\right) \mathrm{e}^{-2 \kappa_{+} t}+\widehat{B}\left(\theta, r_{0}\right)+\theta\left(\mathrm{e}^{-4 \kappa_{+} t}\right), \quad t \rightarrow \infty
$$

Furthermore there exists $k_{0}>0$ such that for all $t>0, \theta \in[0, \pi]$ we have

$$
\left(\frac{\left(r^{2}+a^{2}\right)^{2}}{\sigma^{2}} k^{\prime 2}-\dot{\hat{r}}^{2}\right) \geq k_{0} \mathrm{e}^{-2 \kappa+t}
$$


Proof. - (i) Recall that

$$
\begin{aligned}
& r_{*}=r+\frac{1}{2 \kappa_{+}} \ln \left|r-r_{+}\right|-\frac{1}{2 \kappa_{-}} \ln \left|r-r_{-}\right| \\
& =r-t-\widehat{C}_{1}\left(\theta, r_{0}\right) \mathrm{e}^{-2 \kappa_{+} t}+\widehat{C}_{2}\left(\theta, r_{0}\right)-\frac{1}{2 \kappa_{-}} \ln \left|r-r_{-}\right|+\theta\left(\mathrm{e}^{-4 \kappa_{+} t}\right)
\end{aligned}
$$

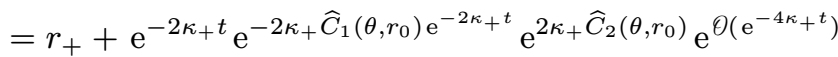

$$
\begin{aligned}
& -\frac{1}{2 \kappa_{-}} \ln \mid \mathrm{e}^{-2 \kappa_{+} t} \mathrm{e}^{2 \kappa_{+} \widehat{C}_{1}\left(\theta, r_{0}\right) \mathrm{e}^{-2 \kappa_{+} t}} \\
& -\mathrm{e}^{2 \kappa_{+} \widehat{C}_{2}\left(\theta, r_{0}\right)} \mathrm{e}^{\vartheta\left(\mathrm{e}^{-4 \kappa_{+} t}\right)}+r_{+}-r_{-} \\
& -t-\widehat{C}_{1}\left(\theta, r_{0}\right) \mathrm{e}^{-2 \kappa_{+} t}+\widehat{C}_{2}\left(\theta, r_{0}\right)+\theta\left(\mathrm{e}^{-4 \kappa_{+} t}\right) \\
& =-t+\mathrm{e}^{-2 \kappa_{+} \widehat{C}_{1}\left(\theta, r_{0}\right)} \mathrm{e}^{2 \kappa_{+} \widehat{C}_{2}\left(\theta, r_{0}\right)} \mathrm{e}^{-2 \kappa_{+} t} \\
& -\frac{1}{2 \kappa_{-}\left(r_{+}-r_{-}\right)} \mathrm{e}^{-2 \kappa_{+} \widehat{C}_{1}\left(\theta, r_{0}\right)} \mathrm{e}^{2 \kappa_{+} \widehat{C}_{2}\left(\theta, r_{0}\right)} \mathrm{e}^{-2 \kappa_{+} t} \\
& +\widehat{C}_{2}\left(\theta, r_{0}\right)-\widehat{C}_{1}\left(\theta, r_{0}\right) \mathrm{e}^{-2 \kappa_{+} t}+r_{+} \\
& -\frac{1}{2 \kappa_{-}} \ln \left|r_{+}-r_{-}\right|+\theta\left(\mathrm{e}^{-4 \kappa_{+} t}\right) \\
& =-t-A\left(\theta, r_{0}\right) \mathrm{e}^{-2 \kappa_{+} t}+B\left(\theta, r_{0}\right)+\theta\left(\mathrm{e}^{-4 \kappa_{+} t}\right),
\end{aligned}
$$

where we have used the Taylor expansions of the functions $\mathrm{e}^{x}$ and $\ln (1+x)$.

(ii) By part (i) of the lemma we have

$$
\begin{aligned}
\frac{\partial \widehat{r}}{\partial t} & =\sqrt{1-\frac{a^{2} \Delta}{\left(r^{2}+a^{2}\right)^{2}}} \frac{\partial r_{*}}{\partial t} \\
& =\sqrt{1-\frac{a^{2} \Delta}{\left(r^{2}+a^{2}\right)^{2}}}\left(-1+2 \kappa_{+} A\left(\theta, r_{0}\right) \mathrm{e}^{-2 \kappa_{+} t}+\theta\left(\mathrm{e}^{-4 \kappa_{+} t}\right)\right) .
\end{aligned}
$$

By Lemma 3.3 we find

$$
\frac{a^{2} \Delta}{\left(r^{2}+a^{2}\right)^{2}}=G\left(r_{0}, \theta\right) \mathrm{e}^{-2 \kappa_{+} t}+\theta\left(\mathrm{e}^{-4 \kappa_{+} t}\right)
$$

and thus

$$
\begin{aligned}
\sqrt{1-\frac{a^{2} \Delta}{\left(r^{2}+a^{2}\right)^{2}}} & =1-\frac{1}{2} \frac{a^{2} \Delta}{\left(r^{2}+a^{2}\right)^{2}}+\Theta\left(\mathrm{e}^{-4 \kappa_{+} t}\right) \\
& =1-\frac{1}{2} G\left(r_{0}, \theta\right) \mathrm{e}^{-2 \kappa_{+} t}+\Theta\left(\mathrm{e}^{-4 \kappa_{+} t}\right) .
\end{aligned}
$$

Putting this into (3.48) gives

$$
\frac{\partial \widehat{r}}{\partial t}=-1+2 \kappa_{+} \widehat{A}\left(\theta, r_{0}\right) \mathrm{e}^{-2 \kappa_{+} t}+\Theta\left(\mathrm{e}^{-4 \kappa_{+} t}\right), \quad t \rightarrow \infty .
$$


It remains to show that $\widehat{A}\left(\theta, r_{0}\right)>0$. The curve $t \mapsto(t, \widehat{r}(t), \theta(t), \varphi(t))$ has to be timelike. Using (3.40) and (3.8) we find

$$
\begin{aligned}
E^{2}=p=\left\langle\gamma_{p}^{\prime}, \gamma_{p}^{\prime}\right\rangle & =\frac{\rho^{2} \Delta}{\left(r^{2}+a^{2}\right)^{2} k^{\prime 2}}\left(\frac{\left(r^{2}+a^{2}\right)^{2}}{\sigma^{2}} k^{2}-\dot{\hat{r}}^{2}\right)\left(\partial_{s} t\right)^{2} \\
& =\frac{E^{2} \sigma^{4}}{\left(r^{2}+a^{2}\right)^{2} k^{\prime 2} \rho^{2} \Delta}\left(\frac{\left(r^{2}+a^{2}\right)^{2}}{\sigma^{2}} k^{\prime 2}-\dot{\hat{r}}^{2}\right) .
\end{aligned}
$$

It follows that

$$
\left(\frac{\left(r^{2}+a^{2}\right)^{2}}{\sigma^{2}} k^{2}-\dot{\hat{r}}^{2}\right) \geq c_{0} \Delta \geq k_{0} \mathrm{e}^{-2 \kappa_{+} t} .
$$

In particular we have $\widehat{A}\left(\theta, r_{0}\right)>0$.

3.2.2. Precise assumptions. - Let us now make the precise assumptions on the collapse. We will suppose that the surface at time $t=0$ is given in the $(t, \widehat{r}, \theta, \varphi)$ coordinate system by $\phi_{0}=\left\{\left(\widehat{r}_{0}\left(\theta_{0}\right), \theta_{0}, \varphi_{0}\right) ;\left(\theta_{0}, \varphi_{0}\right) \in S^{2}\right\}$, where $\widehat{r}_{0}\left(\theta_{0}\right)$ is a smooth function. As $\widehat{r}_{0}$ does not depend on $\varphi_{0}$, we will suppose that $\widehat{z}\left(t, \theta_{0}, \varphi_{0}\right)$ will be independent of $\varphi_{0}: \widehat{z}\left(t, \theta_{0}, \varphi_{0}\right)=\widehat{z}\left(t, \theta_{0}\right)=\widehat{z}(t, \theta)$ as this is the case for $\widehat{r}(t)$ describing the geodesic. Thus the surface of the star is given by

$$
\phi=\left\{(t, \widehat{z}(t, \theta), \omega) ; t \in \mathbb{R}, \omega \in S^{2}\right\} .
$$

The function $\widehat{z}(t, \theta)$ satisfies

$$
\begin{aligned}
& \left\{\begin{array}{l}
\forall t \leq 0, \forall \theta \in[0, \pi], \\
\widehat{z}(t, \theta)=\widehat{z}(0, \theta)<0,
\end{array}\right. \\
& \left\{\begin{array}{l}
\forall t>0, \forall \theta \in[0, \pi], \\
\dot{\widehat{z}}(t, \theta)<0,
\end{array}\right. \\
& \left\{\begin{array}{l}
\exists k_{0}>0, \forall t>0, \forall \theta \in[0, \pi], \\
\left.\left(\frac{\left(r^{2}+a^{2}\right)^{2}}{\sigma^{2}} k^{\prime 2}\right)(\widehat{z}(t, \theta), \theta)-\dot{\widehat{z}}^{2}(t, \theta)\right) \geq k_{0} \mathrm{e}^{-2 \kappa_{+} t},
\end{array}\right. \\
& \left\{\begin{array}{l}
\exists \widehat{A} \in C^{\infty}([0, \pi]), \exists \hat{\xi} \in C^{\infty}(\mathbb{R} \times[0, \pi]), \\
\widehat{z}(t, \theta)=-t-\widehat{A}(\theta) \mathrm{e}^{-2 \kappa_{+} t}+\hat{\xi}(t, \theta), \widehat{A}(\theta)>0, \\
\forall \alpha, \beta, 0 \leq \alpha, \beta \leq 2, \forall \theta \in[0, \pi], \exists C_{\alpha, \beta}, \forall t>0, \\
\left|\partial_{t}^{\alpha} \partial_{\theta}^{\beta} \hat{\xi}(t, \theta)\right| \leq C_{\alpha, \beta} \mathrm{e}^{-4 \kappa_{+} t} .
\end{array}\right.
\end{aligned}
$$

As already explained these assumptions are motivated by the preceding analysis. We do not suppose that a point on the surface moves exactly on a geodesic. Note that (3.52), (3.53) imply

$$
\forall t>0, \forall \theta \in[0, \pi], \quad-1<\dot{\widehat{z}}(t, \theta)<0 .
$$


Equations (3.51)-(3.54) summarize our assumptions on the collapse. The space-time of the collapsing star is given by

$$
\mathcal{M}_{\mathrm{col}}=\{(t, \widehat{r}, \theta, \varphi) ; \widehat{r} \geq \widehat{z}(t, \theta)\} .
$$

We will also note

$$
\Sigma_{t}^{\mathrm{col}}=\{(\widehat{r}, \theta, \varphi) ; \widehat{r} \geq \widehat{z}(t, \theta)\}
$$

Thus

$$
\mathcal{M}_{\mathrm{col}}=\bigcup_{t} \Sigma_{t}^{\mathrm{col}}
$$

Note that in the $\left(t, r_{*}, \theta, \varphi\right)$ coordinate system $\mathcal{M}_{\mathrm{col}}$ and $\Sigma_{t}^{\mathrm{col}}$ are given by

$$
\mathcal{M}_{\mathrm{col}}=\left\{\left(t, r_{*}, \theta, \varphi\right) ; r_{*} \geq z(t, \theta)\right\}, \quad \Sigma_{t}^{\mathrm{col}}=\left\{\left(r_{*}, \theta, \varphi\right) ; r_{*} \geq z(t, \theta)\right\}
$$

with

$$
z(t, \theta)=-t-A(\theta) \mathrm{e}^{-2 \kappa+t}+B(\theta)+\theta\left(\mathrm{e}^{-4 \kappa_{+} t}\right)
$$

for some appropriate $A(\theta), B(\theta)$.

REMARK 3.6. - (i) Let us compare assumptions (3.50)-(3.54) to the preceding discussion on geodesics. Assumption (3.54) contains with respect to the previous discussion an additional asymptotic assumption. Comparing to Lemma 3.4 this condition can be expressed as $\widehat{B}\left(\theta, r_{0}(\theta)\right)=$ Const. $\left(r_{0}(\theta)=r\left(\widehat{r}_{0}(\theta), \theta\right)\right)$. Using the freedom of the constant of integration in (3.4) we can suppose

$$
\widehat{B}\left(\theta, r_{0}(\theta)\right)=0 \text {. }
$$

(ii) The Penrose compactification of block $I$ can be constructed based on the SNG's rather than on the principal null geodesics (PNG's). This construction is explained in Appendix B. Starting from this compactification we could establish a model of the collapsing star that is similar to the one established by Bachelot for the Schwarzschild case (see [6]). In this model the function $\widehat{z}$ would be independent of $\theta$.

We finish this chapter with a lemma which shows that the asymptotic form (3.54) can be accomplished by incoming timelike geodesics with $L=Q=\widetilde{E}=0$.

LEMMA 3.5. - There exists a smooth function $\widehat{r}_{0}(\theta)$ with the following property. Let $\gamma$ be a timelike incoming geodesic with $Q=L=\widetilde{E}=0$ and starting point $\left(0, \widehat{r}_{0}\left(\theta_{0}\right), \theta_{0}, \varphi_{0}\right)$. Then we have along $\gamma$ :

$$
\widehat{r}+t \longrightarrow 0, \quad t \rightarrow \infty
$$

Proof. - Let $M_{\theta}(r)=\widehat{C}_{2}(\theta, r)$ with $\widehat{C}_{2}(\theta, r)$ as in Lemma 3.3. We have (see the explicit form of $\widehat{C}_{2}(\theta, r)$ in the proof of Lemma 3.3):

$$
\lim _{r \rightarrow r_{+}} M_{\theta}(r)=-\infty, \quad \lim _{r \rightarrow \infty} M_{\theta}(r)=\infty, \quad \frac{\partial M_{\theta}}{\partial r} \geq \epsilon>0, \forall r \in\left(r_{+}, \infty\right), \forall \theta \in[0, \pi] .
$$

Therefore $M_{\theta}^{-1}$ exists and we put

$$
r_{0}(\theta)=M_{\theta}^{-1}\left(-a \sin \theta-r_{+}+\frac{1}{2 \kappa_{-}} \ln \left|r_{+}-r_{-}\right|\right), \quad \widehat{r}_{0}(\theta)=\widehat{r}\left(r_{0}(\theta), \theta\right) .
$$


Clearly $\widehat{C}_{2}\left(\theta, r_{0}(\theta)\right)=-a \sin \theta-r_{+}+\frac{1}{2 \kappa_{-}} \ln \left|r_{+}-r_{-}\right|$. Following the proof of Lemma 3.4 we see that

$$
B\left(\theta, r_{0}\right)=\widehat{C}_{2}\left(\theta, r_{0}\right)+r_{+}-\frac{1}{2 \kappa_{-}} \ln \left|r_{+}-r_{-}\right|=-a \sin \theta
$$

Using (3.35), (3.36), (3.38) and (3.47) we see that

$$
\widehat{B}\left(\theta_{0}, r_{0}\left(\theta_{0}\right)\right)=\lim _{t \rightarrow \infty} \widehat{r}+t=B\left(\theta_{0}, r_{0}\left(\theta_{0}\right)\right)+a \sin \theta_{0}=0 .
$$





\section{CHAPTER 4}

\section{CLASSICAL DIRAC FIELDS}

In this chapter we describe classical Dirac fields on $B_{I}$ as well as on $\mathcal{M}_{\text {col }}$. The main results of this chapter are collected in Section 4.1. Sections 4.2 and 4.3 contain a discussion about spin structures and Dirac fields which is valid in general globally hyperbolic space-times. In Section 4.4 we introduce a new Newman-Penrose tetrad which is adapted to our problem and we discuss scattering results as far as they are needed for the formulation and discussion of the main theorem. Other scattering results are collected in Chapter 6. The boundary condition is discussed in Section 4.5. The constructions in this chapter are crucial for what follows. However the reader who wishes to get a first idea of the main theorem can in a first reading accept the results of Section 4.1 and skip the rest of this chapter before coming back to it later.

\subsection{Main results}

Let $\mathcal{H}=L^{2}\left(\left(\mathbb{R}_{\widehat{r}} \times S^{2}, \mathrm{~d} \widehat{r} \mathrm{~d} \omega\right) ; \mathbb{C}^{4}\right), \quad \Gamma^{1}=\operatorname{Diag}(1,-1,-1,1)$.

Proposition 4.1. - There exists a Newman-Penrose tetrad such that the Dirac equation in the Kerr-Newman space-time can be written as

$$
\partial_{t} \psi=i H \psi, \quad H=\Gamma^{1} D_{\widehat{r}}+P_{\omega}+W,
$$

where $W$ is a real potential and $P_{\omega}$ is a differential operator of order one with derivatives only in the angular directions. The operator $H$ is selfadjoint with domain

$$
D(H)=\{v \in \mathscr{H} ; H v \in \mathcal{H}\} .
$$

Proposition 4.2. - There exist selfadjoint operators $P^{ \pm}$such that for all $g \in C_{\infty}(\mathbb{R})$ :

$$
g\left(P^{ \pm}\right)=s-\lim _{t \rightarrow \pm \infty} \mathrm{e}^{-i t H} g\left(\frac{\widehat{r}}{t}\right) \mathrm{e}^{i t H}
$$


Let

$$
\begin{aligned}
& H_{\leftarrow}=\Gamma^{1} D_{\widehat{r}}-\frac{a}{r_{+}^{2}+a^{2}} D_{\varphi}-\frac{q Q r_{+}}{r_{+}^{2}+a^{2}}, \\
& \mathscr{H}^{+}=\left\{v=\left(v_{1}, v_{2}, v_{3}, v_{4}\right) \in \mathscr{H} ; v_{1}=v_{4}=0\right\}, \\
& \mathscr{H}^{-}=\left\{v=\left(v_{1}, v_{2}, v_{3}, v_{4}\right) \in \mathscr{H} ; v_{2}=v_{3}=0\right\} .
\end{aligned}
$$

The operator $H_{\leftarrow}$ is selfadjoint on $\mathcal{H}$ with domain $D\left(H_{\leftarrow}\right)=\left\{v \in \mathscr{H} ; H_{\leftarrow} v \in \mathcal{H}\right\}$.

THEOREM 4.1. - The following wave operators exist:

$$
\begin{aligned}
& W_{\leftarrow}^{ \pm}=s-\lim _{t \rightarrow \pm \infty} \mathrm{e}^{-i t H} \mathrm{e}^{i t H_{\leftarrow}} P_{\mathcal{H}^{\mp}}, \\
& \Omega_{\leftarrow}^{ \pm}=s-\lim _{t \rightarrow \pm \infty} \mathrm{e}^{-i t H \leftarrow} \mathrm{e}^{i t H} \mathbf{1}_{\mathbb{R}^{\mp}}\left(P^{ \pm}\right) .
\end{aligned}
$$

There exist similar wave operators at infinity using a modified asymptotic dynamics $U_{D}(t)$. Using the above tetrad the Dirac equation with MIT boundary condition (chiral angle $\nu$ ) can be written in the form

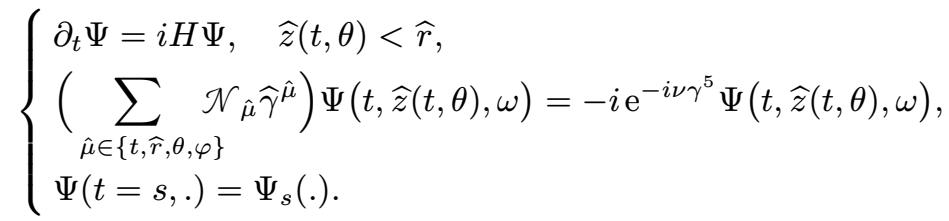

Here $\mathcal{N}_{\hat{\mu}}$ are the coordinates of the conormal, $\widehat{\gamma}^{\hat{\mu}}$ are some appropriate Dirac matrices and $\gamma^{5}=\operatorname{Diag}(1,1,-1,-1)$. Let $\mathscr{H}_{t}=L^{2}\left(\left(\left\{(\widehat{r}, \omega) \in \mathbb{R} \times S^{2} ; \widehat{r} \geq \widehat{z}(t, \theta)\right\}, \mathrm{d} \widehat{r} \mathrm{~d} \omega\right) ; \mathbb{C}^{4}\right)$.

Proposition 4.3. - Equation (4.2) can be solved by a unitary propagator

$$
U(t, s): \mathscr{H}_{s} \longrightarrow \mathcal{H}_{t} \text {. }
$$

\subsection{Spin structures}

Let $(\mathcal{M}, g)$ be a smooth 4-manifold with a lorentzian metric $g$ with signature $(+,-,-,-)$ which is assumed to be oriented, time oriented and globally hyperbolic. Global hyperbolicity implies:

1) $(\mathcal{M}, g)$ admits a spin structure (see R.P. Geroch [24], [25], [26] and E. Stiefel [45]) and we choose one. We denote by $\mathbb{S}$ (or $\mathbb{S}^{A}$ in the abstract index formalism) the spin bundle over $\mathcal{M}$ and $\overline{\mathbb{S}}$ (or $\mathbb{S}^{A^{\prime}}$ ) the same bundle with the complex structure replaced by its opposite. The dual bundles $\mathbb{S}^{*}$ and $\overline{\mathbb{S}}^{*}$ will be denoted respectively $\mathbb{S}_{A}$ and $\mathbb{S}_{A^{\prime}}$. The complexified tangent bundle to $\mathcal{M}$ is recovered as the tensor product of $\mathbb{S}$ and $\overline{\mathbb{S}}$, i.e.

$$
T \mathcal{M} \otimes \mathbb{C}=\mathbb{S} \otimes \overline{\mathbb{S}} \quad \text { or } \quad T^{a} \mathcal{M} \otimes \mathbb{C}=\mathbb{S}^{A} \otimes \mathbb{S}^{A^{\prime}}
$$

and similarly

$$
T^{*} \mathcal{M} \otimes \mathbb{C}=\mathbb{S}^{*} \otimes \overline{\mathbb{S}}^{*} \quad \text { or } \quad T_{a} \mathcal{M} \otimes \mathbb{C}=\mathbb{S}_{A} \otimes \mathbb{S}_{A^{\prime}}
$$


An abstract tensor index $a$ is thus understood as an unprimed spinor index $A$ and a primed spinor index $A^{\prime}$ clumped together: $a=A A^{\prime}$. The symplectic forms on $\mathbb{S}$ and $\overline{\mathbb{S}}$ are denoted $\epsilon_{A B}, \epsilon_{A^{\prime} B^{\prime}}$ and are referred to as the Levi-Civita symbols. The form $\epsilon_{A B}$ can be seen as an isomorphism from $\mathbb{S}$ to $\mathbb{S}^{*}$ which to $\kappa^{A}$ associates $\kappa_{A}=\kappa^{B} \epsilon_{B A}$. Similarly, $\epsilon_{A^{\prime} B^{\prime}}$ and the corresponding $\epsilon^{A^{\prime} B^{\prime}}$ can be regarded as lowering and raising devices for primed indices. The metric $g$ is expressed in terms of the Levi-Civita symbols as $g_{\mathrm{ab}}=\epsilon_{A B} \epsilon_{A^{\prime} B^{\prime}}$.

2) There exists a global time function $t$ on $\mathcal{M}$. The level surfaces $\Sigma_{t}, t \in \mathbb{R}$, of the function $t$ define a foliation of $\mathcal{M}$, all $\Sigma_{t}$ being Cauchy surfaces and homeomorphic to a given smooth 3 -manifold $\Sigma$ (see Geroch [26]). Geroch's theorem does not say anything about the regularity of the leaves $\Sigma_{t}$; the time function is only proved to be continuous and they are thus simply understood as topological submanifolds of $\mathcal{M}$. A regularization procedure for the time function can be found in [8], [9]. In the concrete cases which we consider in this paper the time function is smooth and all the leaves are diffeomorphic to $\Sigma$. The function $t$ is then a smooth time coordinate on $\mathcal{M}$ and it is increasing along any non space-like future oriented curve. Its gradient $\nabla^{a} t$ is everywhere orthogonal to the level surfaces $\Sigma_{t}$ of $t$ and it is therefore everywhere timelike; it is also future oriented. We identify $\mathcal{M}$ with the smooth manifold $\mathbb{R} \times \Sigma$ and consider $g$ as a tensor valued function on $\mathbb{R} \times \Sigma$.

Let $T^{a}$ be the future-pointing timelike vector field normal to $\Sigma_{t}$, normalized for later convenience to satisfy $T^{a} T_{a}=2$, i.e.

$$
T^{a}=\frac{\sqrt{2}}{|\nabla t|} \nabla^{a} t, \quad \text { where } \quad|\nabla t|=\left(g_{\mathrm{ab}} \nabla^{a} t \nabla^{b} t\right)^{\frac{1}{2}} .
$$

\subsection{The Dirac equation and the Newman-Penrose formalism}

In terms of two component spinors (sections of the bundles $\mathbb{S}^{A}, \mathbb{S}_{A}, \mathbb{S}^{A^{\prime}}$ or $\mathbb{S}_{A^{\prime}}$ ), the charged Dirac equation takes the form (see [44], page 418):

$$
\left\{\begin{array}{l}
\left(\nabla_{A^{\prime}}^{A}-i q \Phi_{A^{\prime}}^{A}\right) \phi_{A}=\mu \chi_{A^{\prime}}, \\
\left(\nabla_{A}^{A^{\prime}}-i q \Phi_{A}^{A^{\prime}}\right) \chi_{A^{\prime}}=\mu \phi_{A}, \quad \mu=m / \sqrt{2},
\end{array}\right.
$$

where $m \geq 0$ is the mass of the field. The Dirac equation (4.3) possesses a conserved current (see for example [40]) on general curved space-times, defined by the future oriented non-spacelike vector field, sum of two future oriented null vector fields

$$
V^{a}=\phi^{A} \bar{\phi}^{A^{\prime}}+\bar{\chi}^{A} \chi^{A^{\prime}}
$$

The vector field $V^{a}$ is divergence free, i.e. $\nabla^{a} V_{a}=0$. Consequently the 3 -form $\omega=$ $* V_{a} \mathrm{~d} x^{a}$ is closed. Let $\Sigma$ be a spacelike or characteristic hypersurface, $\mathrm{d} \Omega$ the volume 
form on $\mathcal{M}$ induced by the metric ( $\mathrm{d} \Omega=\rho^{2} \mathrm{~d} t \wedge \mathrm{d} r \wedge \mathrm{d} \omega$ for the Kerr-Newman metric), $\mathcal{N}^{a}$ the (future pointing) normal to $\Sigma$ and $\mathscr{L}^{a}$ transverse to $\Sigma$ with $\mathcal{N}^{a} \mathscr{L}_{a}=1$. Then

$$
\begin{gathered}
\int_{\Sigma} *\left(\phi_{A} \bar{\phi}_{A^{\prime}} \mathrm{d} x^{A A^{\prime}}+\bar{\chi}_{A} \chi_{A^{\prime}} \mathrm{d} x^{A A^{\prime}}\right) \\
=\int_{\Sigma} \mathcal{N}_{B B^{\prime}} \mathscr{L}^{B B^{\prime}} *\left(\phi_{A} \bar{\phi}_{A^{\prime}} \mathrm{d} x^{A A^{\prime}}+\bar{\chi}_{A} \chi_{A^{\prime}} \mathrm{d} x^{A A^{\prime}}\right) \\
\left.=\int_{\Sigma} \mathscr{L}^{C C^{\prime}} \partial_{C C^{\prime}}\right\lrcorner\left(\mathcal{N}_{B B^{\prime}} \mathrm{d} x^{B B^{\prime}} \wedge *\left(\phi_{A} \bar{\phi}_{A^{\prime}} \mathrm{d} x^{A A^{\prime}}+\bar{\chi}_{A} \chi_{A^{\prime}} \mathrm{d} x^{A A^{\prime}}\right)\right) \\
\left.=\int_{\Sigma} \mathcal{N}^{A A^{\prime}}\left(\phi_{A} \bar{\phi}_{A^{\prime}}+\bar{\chi}_{A} \chi_{A^{\prime}}\right)\left(\mathscr{L}^{B B^{\prime}} \partial_{B B^{\prime}}\right)\right\lrcorner \mathrm{d} \Omega .
\end{gathered}
$$

If $\Sigma$ is spacelike we can take $\mathcal{L}^{A A^{\prime}}=\mathcal{N}^{A A^{\prime}}$ and the integral defines a norm and by this norm the space $L^{2}\left(\Sigma ; \mathbb{S}_{A} \oplus \mathbb{S}^{A^{\prime}}\right)$ as completion of $C_{0}^{\infty}\left(\Sigma ; \mathbb{S}_{A} \oplus \mathbb{S}^{A^{\prime}}\right)$. Note that if $\Sigma$ is characteristic $\int_{\Sigma} * \phi_{A} \bar{\phi}_{A^{\prime}} \mathrm{d} x^{A A^{\prime}}=0$ does not entail $\phi_{A}=0$ on $\Sigma$ (see Remark 4.1). If $\Sigma_{t}$ are the level surfaces of $t$, then we see by Stokes' theorem that the total charge

$$
C(t)=\frac{1}{\sqrt{2}} \int_{\Sigma_{t}} V_{a} T^{a} \mathrm{~d} \sigma_{\Sigma_{t}}
$$

is constant throughout time. Here $\left.\mathrm{d} \sigma_{\Sigma_{t}}=(1 / \sqrt{2}) T^{a}\right\lrcorner \mathrm{d} \Omega$.

Using the Newman-Penrose formalism, equation (4.3) can be expressed as a system of partial differential equations with respect to a coordinate basis. This formalism is based on the choice of a null tetrad, i.e. a set of four vector fields $\ell^{a}, n^{a}, m^{a}$ and $\bar{m}^{a}$, the first two being real and future oriented, $\bar{m}^{a}$ being the complex conjugate of $m^{a}$, such that all four vector fields are null and $m^{a}$ is orthogonal to $\ell^{a}$ and $n^{a}$, that is to say

$$
\ell_{a} \ell^{a}=n_{a} n^{a}=m_{a} m^{a}=\ell_{a} m^{a}=n_{a} m^{a}=0 .
$$

The tetrad is said to be normalized if in addition

$$
\ell_{a} n^{a}=1, \quad m_{a} \bar{m}^{a}=-1 .
$$

The vectors $\ell^{a}$ and $n^{a}$ usually describe "dynamic" or scattering directions, i.e. directions along which light rays may escape towards infinity (or more generally asymptotic regions corresponding to scattering channels). The vector $m^{a}$ tends to have, at least spatially, bounded integral curves, typically $m^{a}$ and $\bar{m}^{a}$ generate rotations. The principle of the Newman-Penrose formalism is to decompose the covariant derivative into directional covariant derivatives along the frame vectors. We introduce a spinframe $\left\{o^{A}, \iota^{A}\right\}$, defined uniquely up to an overall sign factor by the requirements that

$$
o^{A} \bar{o}^{A^{\prime}}=\ell^{a}, \quad \iota^{A} \bar{\iota}^{A^{\prime}}=n^{a}, \quad o^{A} \bar{\iota}^{A^{\prime}}=m^{a}, \quad \iota^{A} \bar{o}^{A^{\prime}}=\bar{m}^{a}, \quad o_{A} \iota^{A}=1 .
$$

We will also denote the spin frame by $\left\{\epsilon_{0}^{A}, \epsilon_{1}^{A}\right\}$. The dual basis of $\mathbb{S}_{A}$ is $\left\{\epsilon_{A}^{0}, \epsilon_{A}^{1}\right\}$, where $\epsilon_{A}^{0}=-\iota_{A}, \epsilon_{A}^{1}=o_{A}$. Let $\phi_{0}$ and $\phi_{1}$ be the components of $\phi_{A}$ in $\left\{o^{A}, \iota^{A}\right\}$, and $\chi_{0^{\prime}}, \chi_{1^{\prime}}$ 
the components of $\chi_{A^{\prime}}$ in $\left(\bar{o}^{A^{\prime}}, \bar{\iota}^{A^{\prime}}\right)$ :

$$
\phi_{0}=\phi_{A} o^{A}, \quad \phi_{1}=\phi_{A} \iota^{A}, \quad \chi_{0^{\prime}}=\chi_{A^{\prime}} \bar{o}^{A^{\prime}}, \quad \chi_{1^{\prime}}=\chi_{A^{\prime}} \bar{\iota}^{A^{\prime}} .
$$

The Dirac equation then takes the form (see for example [13])

$$
\left\{\begin{array}{l}
n^{\boldsymbol{a}}\left(\partial_{\boldsymbol{a}}-i q \Phi_{\boldsymbol{a}}\right) \phi_{0}-m^{\boldsymbol{a}}\left(\partial_{\boldsymbol{a}}-i q \Phi_{\boldsymbol{a}}\right) \phi_{1}+(\mu-\gamma) \phi_{0}+(\tau-\beta) \phi_{1}=\frac{m}{\sqrt{2}} \chi_{1^{\prime}}, \\
\ell^{\boldsymbol{a}}\left(\partial_{\boldsymbol{a}}-i q \Phi_{\boldsymbol{a}}\right) \phi_{1}-\bar{m}^{\boldsymbol{a}}\left(\partial_{\boldsymbol{a}}-i q \Phi_{\boldsymbol{a}}\right) \phi_{0}+(\alpha-\pi) \phi_{0}+(\varepsilon-\tilde{\rho}) \phi_{1}=-\frac{m}{\sqrt{2}} \chi_{0^{\prime}}, \\
n^{\boldsymbol{a}}\left(\partial_{\boldsymbol{a}}-i q \Phi_{\boldsymbol{a}}\right) \chi_{0^{\prime}}-\bar{m}^{\boldsymbol{a}}\left(\partial_{\boldsymbol{a}}-i q \Phi_{\boldsymbol{a}}\right) \chi_{1^{\prime}}+(\bar{\mu}-\bar{\gamma}) \chi_{0^{\prime}}+(\bar{\tau}-\bar{\beta}) \chi_{1^{\prime}}=\frac{m}{\sqrt{2}} \phi_{1}, \\
\ell^{\boldsymbol{a}}\left(\partial_{\boldsymbol{a}}-i q \Phi_{\boldsymbol{a}}\right) \chi_{1^{\prime}}-m^{\boldsymbol{a}}\left(\partial_{\boldsymbol{a}}-i q \Phi_{\boldsymbol{a}}\right) \chi_{0^{\prime}}+(\bar{\alpha}-\bar{\pi}) \chi_{0^{\prime}}+(\bar{\varepsilon}-\overline{\tilde{\rho}}) \chi_{1^{\prime}}=-\frac{m}{\sqrt{2}} \phi_{0} .
\end{array}\right.
$$

The $\mu, \gamma$, etc. are the so called spin coefficients, for example $\mu=-\bar{m}^{a} \delta n_{a}$ and $\delta=$ $m^{a} \nabla_{a}$. For the formulas of the spin coefficients and details about the Newman-Penrose formalism see e.g. [44].

It is often useful to allow simultaneous consideration of bases of $T^{a} \mathcal{M}$ and $\mathbb{S}^{A}$, which are completely unrelated to one another. Let $\left\{e_{0}, e_{1}, e_{2}, e_{3}\right\}$ be such a basis of $T^{a} \mathcal{M}$, which is not related to the Newman-Penrose tetrad.

We define the Infeld-Van der Waerden symbols as the spinor components of the frame vectors in the spin frame $\left\{\epsilon_{0}^{A}, \epsilon_{1}^{A}\right\}$ :

$$
g_{\boldsymbol{a}}^{\boldsymbol{A} \boldsymbol{A}^{\prime}}=e_{\boldsymbol{a}}^{\boldsymbol{A} \boldsymbol{A}^{\prime}}=g_{\boldsymbol{a}}^{a} \epsilon_{A}^{\boldsymbol{A}} \epsilon_{A^{\prime}}^{\boldsymbol{A}^{\prime}}=\left(\begin{array}{rr}
n_{\boldsymbol{a}} & -\bar{m}_{\boldsymbol{a}} \\
-m_{\boldsymbol{a}} & \ell_{\boldsymbol{a}}
\end{array}\right)
$$

(recall that $g_{\boldsymbol{a}}^{a}=e_{\boldsymbol{a}}^{a}$ denotes the vector field $e_{\boldsymbol{a}}$ ). We use these quantities to express (4.3) in terms of spinor components:

$$
\left\{\begin{array}{c}
-i \epsilon_{A^{\prime}}^{\boldsymbol{A}^{\prime}}\left(\nabla^{A A^{\prime}}-i q \Phi^{A A^{\prime}}\right) \phi_{A}=-i g^{\boldsymbol{a} \boldsymbol{A} \boldsymbol{A}^{\prime}} \epsilon_{\boldsymbol{A}}^{A}\left(\nabla_{\boldsymbol{a}}-i q \Phi_{\boldsymbol{a}}\right) \phi_{A}=-i \mu \chi^{\boldsymbol{A}^{\prime},} \\
-i \epsilon_{\boldsymbol{A}}^{A}\left(\nabla_{A A^{\prime}}-i q \Phi_{A A^{\prime}}\right) \chi^{A^{\prime}}=-i g_{\boldsymbol{A} \boldsymbol{A}^{\prime}}^{\boldsymbol{a}} \epsilon_{A^{\prime}}^{\boldsymbol{A}^{\prime}}\left(\nabla_{\boldsymbol{a}}-i q \Phi_{\boldsymbol{a}}\right) \chi^{A^{\prime}}=i \mu \phi_{\boldsymbol{A}},
\end{array}\right.
$$

where $\nabla_{\boldsymbol{a}}$ denotes $\nabla_{e_{\boldsymbol{a}}}$. For $\boldsymbol{a}=0,1,2,3$, we introduce the $2 \times 2$ matrices

$$
A^{\boldsymbol{a}}={ }^{t} g^{\boldsymbol{a} \boldsymbol{A A ^ { \prime }}}, \quad B^{\boldsymbol{a}}=g_{\boldsymbol{A} \boldsymbol{A}^{\prime}}^{\boldsymbol{a}},
$$

and the $4 \times 4$ matrices

$$
\gamma^{a}=\left(\begin{array}{ll}
0 & i \sqrt{2} B^{a} \\
-i \sqrt{2} A^{a} & 0
\end{array}\right)
$$

We find

$$
\gamma^{a}=i \sqrt{2}\left(\begin{array}{cccc}
0 & 0 & \ell^{\boldsymbol{a}} & m^{\boldsymbol{a}} \\
0 & 0 & \bar{m}^{\boldsymbol{a}} & n^{\boldsymbol{a}} \\
-n^{\boldsymbol{a}} & m^{\boldsymbol{a}} & 0 & 0 \\
\bar{m}^{\boldsymbol{a}} & -\ell^{\boldsymbol{a}} & 0 & 0
\end{array}\right) .
$$


Putting $\Psi=\phi_{A} \oplus \chi^{A^{\prime}}$, the components of $\Psi$ in the spin frame are

$$
\boldsymbol{\Psi}={ }^{t}\left(\phi_{0}, \phi_{1}, \chi^{0^{\prime}}, \chi^{1^{\prime}}\right)
$$

and (4.9) becomes

$$
\sum_{\boldsymbol{a}=0}^{3} \gamma^{\boldsymbol{a}} \mathscr{P}\left(\nabla_{e_{\boldsymbol{a}}}-i q \Phi_{\boldsymbol{a}}\right) \Psi+i m \boldsymbol{\Psi}=0,
$$

where $\mathscr{P}$ is the mapping that to a Dirac spinor associates its components in the spin frame:

$$
\Psi=\phi_{A} \oplus \chi^{A^{\prime}} \mapsto \Psi=\phi_{\boldsymbol{A}} \oplus \chi^{\boldsymbol{A}^{\prime}}
$$

REMARK 4.1. - We have

$$
\phi_{A}=-\phi_{0} \iota_{A}+\phi_{1} o_{A}, \quad \chi_{A^{\prime}}=-\chi_{0^{\prime}} \bar{\iota}_{A^{\prime}}+\chi_{1^{\prime}} \bar{o}_{A^{\prime}} .
$$

Thus

$$
\begin{aligned}
\phi_{A} \bar{\phi}_{A^{\prime}} & =\left(-\phi_{0} \iota_{A}+\phi_{1} o_{A}\right)\left(-\bar{\phi}_{0} \bar{\iota}_{A^{\prime}}+\bar{\phi}_{1} \bar{o}_{A^{\prime}}\right) \\
& =\left|\phi_{0}\right|^{2} n_{a}-\phi_{0} \bar{\phi}_{1} \bar{m}_{a}-\phi_{1} \bar{\phi}_{0} m_{a}+\left|\phi_{1}\right|^{2} \ell_{a}, \\
\bar{\chi}_{A} \chi_{A^{\prime}} & =\left|\chi_{0^{\prime}}\right|^{2} n_{a}-\bar{\chi}_{0^{\prime}} \chi_{1^{\prime}} \bar{m}_{a}-\bar{\chi}_{1^{\prime}} \chi_{0^{\prime}} m_{a}+\left|\chi_{1^{\prime}}\right|^{2} \ell_{a} .
\end{aligned}
$$

Putting $\Psi=\left(\phi_{0}, \phi_{1}, \chi_{1^{\prime}},-\chi_{0^{\prime}}\right)$ we obtain

$$
\begin{aligned}
\phi_{A} \bar{\phi}_{A^{\prime}}+\bar{\chi}_{A} \chi_{A^{\prime}}=\left|\Psi_{1}\right|^{2} n_{a}- & \Psi_{1} \bar{\Psi}_{2} \bar{m}_{a}-\Psi_{2} \bar{\Psi}_{1} m_{a}+\left|\Psi_{2}\right|^{2} \ell_{a} \\
& +\left|\Psi_{4}\right|^{2} n_{a}+\bar{\Psi}_{4} \Psi_{3} \bar{m}_{a}+\bar{\Psi}_{3} \Psi_{4} m_{a}+\left|\Psi_{3}\right|^{2} \ell_{a} .
\end{aligned}
$$

Thus for a vector field $X^{a}$ we have

$$
\begin{gathered}
X^{A A^{\prime}}\left(\phi_{A} \bar{\phi}_{A^{\prime}}+\bar{\chi}_{A} \chi_{A^{\prime}}\right)=\langle\mathbf{X} \Psi, \Psi\rangle_{\mathbb{C}^{4}}, \\
\mathbf{X}=\left(\begin{array}{cccc}
n_{a} X^{a} & -m_{a} X^{a} & 0 & 0 \\
-\bar{m}_{a} X^{a} & \ell_{a} X^{a} & 0 & 0 \\
0 & 0 & \ell_{a} X^{a} & m_{a} X^{a} \\
0 & 0 & \bar{m}_{a} X^{a} & n_{a} X^{a}
\end{array}\right) .
\end{gathered}
$$

If $\Sigma$ is a characteristic hypersurface with conormal $n_{a}$, then

$$
\begin{aligned}
\int_{\Sigma} *\left(\left(\phi_{A} \bar{\phi}_{A^{\prime}}\right.\right. & \left.\left.+\bar{\chi}_{A} \chi_{A^{\prime}}\right) \mathrm{d} x^{A A^{\prime}}\right) \\
& =\int_{\Sigma} n^{a}\left(\phi_{A} \bar{\phi}_{A^{\prime}}+\bar{\chi}_{A} \chi_{A^{\prime}}\right) \mathrm{d} \sigma_{\Sigma}=\int_{\Sigma}\left(\left|\Psi_{2}\right|^{2}+\left|\Psi_{3}\right|^{2}\right) \mathrm{d} \sigma_{\Sigma},
\end{aligned}
$$

where $\left.\mathrm{d} \sigma_{\Sigma}=\left(\ell^{a} \partial_{a}\right)\right\lrcorner \mathrm{d} \Omega$. 


\subsection{The Dirac equation on block $I$}

4.4.1. A new Newman-Penrose tetrad. - The tetrad normally used to describe the Dirac equation on the Kerr-Newman background is Kinnersley's tetrad (see [34]). Kinnersley uses the type D structure of the space-time and chooses the vectors $n^{a}$, $\ell^{a}$ to be $V^{ \pm}$. In [29] it is argued that this tetrad is not adapted to time dependent scattering problems. We introduce a new tetrad $L^{a}, N^{a}, M^{a}, \bar{M}^{a}$ which is adapted to the foliation in the sense that

$$
L^{a}+N^{a}=T^{a} .
$$

One advantage of a tetrad adapted to the foliation is that the conserved current simply reads

$$
\frac{1}{\sqrt{2}} \int_{\Sigma_{t}}\left(\left|\phi_{0}\right|^{2}+\left|\phi_{1}\right|^{2}+\left|\chi_{0^{\prime}}\right|^{2}+\left|\chi_{1^{\prime}}\right|^{2}\right) \mathrm{d} \sigma_{\Sigma_{t}} .
$$

This follows from the formulas (4.4) and (4.13). We choose $L^{a}$ and $N^{a}$ in the plane spanned by $T^{a}$ and $\partial_{r}$ and $L^{a}$ to be outgoing, $N^{a}$ to be incoming. The choice of $M^{a}$ is then imposed, except for the freedom of a constant factor of modulus 1. In [29] this construction is done for the Kerr metric, the corresponding tetrad for the KerrNewman metric is calculated in [15]:

$$
\left\{\begin{aligned}
L^{a} & =\frac{1}{2} T^{a}+\sqrt{\frac{\Delta}{2 \rho^{2}}} \partial_{r}=\sqrt{\frac{\sigma^{2}}{2 \Delta \rho^{2}}}\left(\partial_{t}+\frac{a\left(2 M r-Q^{2}\right)}{\sigma^{2}} \partial_{\varphi}\right)+\sqrt{\frac{\Delta}{2 \rho^{2}}} \partial_{r}, \\
N^{a} & =\frac{1}{2} T^{a}-\sqrt{\frac{\Delta}{2 \rho^{2}}} \partial_{r}=\sqrt{\frac{\sigma^{2}}{2 \Delta \rho^{2}}}\left(\partial_{t}+\frac{a\left(2 M r-Q^{2}\right)}{\sigma^{2}} \partial_{\varphi}\right)-\sqrt{\frac{\Delta}{2 \rho^{2}}} \partial_{r}, \\
M^{a} & =\frac{1}{\sqrt{2 \rho^{2}}}\left(\partial_{\theta}+\frac{\rho^{2}}{\sqrt{\sigma^{2}}} \frac{i}{\sin \theta} \partial_{\varphi}\right) .
\end{aligned}\right.
$$

The Dirac equation in the Kerr-Newman metric is then described in the following way. Let

$$
\mathbb{D}_{S^{2}}=\frac{1}{i}\left(\begin{array}{cc}
0 & \partial_{\theta}+\frac{\cot \theta}{2}+\frac{i}{\sin \theta} \partial_{\varphi} \\
\partial_{\theta}+\frac{\cot \theta}{2}-\frac{i}{\sin \theta} \partial_{\varphi} & 0
\end{array}\right), \quad \not D_{S^{2}}=\left(\begin{array}{cc}
\mathbb{D}_{S^{2}} & 0 \\
0 & -\mathbb{D}_{S^{2}}
\end{array}\right) .
$$

We define the Pauli matrices

$$
\sigma^{0}=\left(\begin{array}{ll}
1 & 0 \\
0 & 1
\end{array}\right), \quad \sigma^{1}=\left(\begin{array}{rr}
1 & 0 \\
0 & -1
\end{array}\right), \quad \sigma^{2}=\left(\begin{array}{ll}
0 & 1 \\
1 & 0
\end{array}\right), \quad \sigma^{3}=i\left(\begin{array}{rr}
0 & -1 \\
1 & 0
\end{array}\right)
$$

and the Dirac matrices

$$
\gamma^{0}=i\left(\begin{array}{cc}
0 & \sigma^{0} \\
-\sigma^{0} & 0
\end{array}\right), \quad \gamma^{k}=i\left(\begin{array}{cc}
0 & \sigma^{k} \\
\sigma^{k} & 0
\end{array}\right), \quad k=1,2,3,
$$

which satisfy the anticommutation relations

$$
\gamma^{\mu} \gamma^{\nu}+\gamma^{\nu} \gamma^{\mu}=2 \eta^{\mu \nu} I_{\mathbb{R}^{4}}, \quad \mu, \nu=0, \ldots, 3, \quad \eta^{\mu \nu}=\operatorname{Diag}(1,-1,-1,-1) .
$$


Let

$$
\gamma^{5}:=-i \gamma^{0} \gamma^{1} \gamma^{2} \gamma^{3}=\operatorname{Diag}(1,1,-1,-1)
$$

We will also need the matrices $\Gamma^{j}(j=1, \ldots, 4)$ defined by

$$
\Gamma^{k}=\left(\begin{array}{cc}
\sigma^{k} & 0 \\
0 & -\sigma^{k}
\end{array}\right) \quad(1 \leq k \leq 3), \quad \Gamma^{4}=i\left(\begin{array}{cc}
0 & -\sigma^{0} \\
\sigma^{0} & 0
\end{array}\right)
$$

Note that

$$
\Gamma^{1}=\operatorname{Diag}(1,-1,-1,1) .
$$

Let now $\Phi$ be the bi-spinor ${ }^{t}\left(\phi_{0}, \phi_{1}, \chi_{1^{\prime}},-\chi_{0^{\prime}}\right)$ and

$$
\Psi=\sqrt{\frac{\sqrt{\Delta} \sigma \rho}{r^{2}+a^{2}}} \Phi
$$

Then the equation satisfied by $\Psi$ is (see [15]):

$$
\begin{aligned}
& \partial_{t} \Psi=i \not D \Psi, \\
& \not D=h D_{\mathfrak{s}} h+V_{\varphi} D_{\varphi}+V_{1}, \\
& \not D_{\mathfrak{s}}=\Gamma^{1} D_{r_{*}}+a_{0}\left(r_{*}\right) \not D_{S^{2}}+b_{0}\left(r_{*}\right) \Gamma^{4}+c_{1}\left(r_{*}\right)+c_{2}^{\varphi}\left(r_{*}\right) D_{\varphi}, \\
& a_{0}\left(r_{*}\right)=\frac{\sqrt{\Delta}}{r^{2}+a^{2}}, \quad b_{0}\left(r_{*}\right)=\frac{m \sqrt{\Delta}}{\sqrt{r^{2}+a^{2}}}, c_{1}\left(r_{*}\right)=-\frac{q Q r}{r^{2}+a^{2}}, \\
& c_{2}^{\varphi}\left(r_{*}\right)=-\frac{a\left(2 M r-Q^{2}\right)}{\left(r^{2}+a^{2}\right)^{2}}, \\
& h\left(r_{*}, \theta\right)=\sqrt{\frac{r^{2}+a^{2}}{\sigma}}, \\
& V_{\varphi}=-\frac{\sqrt{\Delta}}{\sigma \sin \theta}\left(\frac{\rho^{2}}{\sigma}-1\right) \Gamma^{3}-\frac{a\left(2 M r-Q^{2}\right)}{\left(r^{2}+a^{2}\right) \sigma^{2}}\left(r^{2}+a^{2}-\sigma\right), \\
& V_{1}=\mathbb{V}_{0}+\frac{m \sqrt{\Delta}}{\sigma}\left(\rho-\sqrt{r^{2}+a^{2}}\right) \Gamma^{4}-\frac{q Q r}{\sigma^{2}}\left(r^{2}+a^{2}-\sigma\right), \\
& \mathbb{V}_{0}=\left(\begin{array}{rrrr}
v_{0} & v_{1} & 0 & 0 \\
\bar{v}_{1} & -v_{0} & 0 & 0 \\
0 & 0 & v_{0} & \bar{v}_{1} \\
0 & 0 & v_{1} & -v_{0}
\end{array}\right),
\end{aligned}
$$




$$
\begin{aligned}
v_{0}=- & \frac{a^{3}\left(2 M r-Q^{2}\right) \Delta \sin ^{2} \theta \cos \theta}{2 \rho^{2} \sigma^{3}}, \\
v_{1}=- & \frac{a M \sqrt{\Delta} \sin \theta}{2 \rho^{2} \sigma} \\
& +\frac{a \sqrt{\Delta} \sin \theta\left(2 M r-Q^{2}\right)\left(2 r\left(r^{2}+a^{2}\right)-(r-M) a^{2} \sin ^{2} \theta\right)}{2 \rho^{2} \sigma^{3}} \\
& -i \frac{q Q \sqrt{\Delta} r a \sin \theta}{\sigma^{2}} .
\end{aligned}
$$

Recall from [15] that $\not D_{\mathfrak{s}}, \not D$ are selfadjoint on

$$
\mathcal{H}_{*}:=L^{2}\left(\left(\mathbb{R} \times S^{2} ; \mathrm{d} r_{*} \mathrm{~d} \omega\right) ; \mathbb{C}^{4}\right)
$$

with domain

$$
\mathcal{H}_{*}^{1}:=D\left(\not D_{\mathfrak{s}}\right)=D(\not D)=\left\{u \in \mathscr{H}_{*} ; \not D u \in \mathcal{H}_{*}\right\}
$$

and that their spectrum is purely absolutely continuous. We also define the asymptotic dynamics

$$
\begin{aligned}
& \not_{H}=\Gamma^{1} D_{r_{*}}-\frac{a}{r_{+}^{2}+a^{2}} D_{\varphi}-\frac{q Q r_{+}}{r_{+}^{2}+a^{2}}, \quad D\left(\not_{H}\right)=\left\{u \in \mathcal{H}_{*} ; \not_{H} u \in \mathcal{H}_{*}\right\} \\
& \not_{\rightarrow}=\Gamma^{1} D_{r_{*}}+m \Gamma^{4}, D\left(\not_{\rightarrow}\right)=\left\{u \in \mathcal{H}_{*} ; \not_{\rightarrow} u \in \mathcal{H}_{*}\right\} .
\end{aligned}
$$

Even if the above tetrad was successfully used for the proof of the asymptotic completeness result, it has a major drawback for the treatment of the Hawking effect. In fact in this representation and using the Regge-Wheeler type coordinate $r_{*}$ the modulus of the local velocity

$$
v:=\left[r_{*}, \not D\right]=h^{2} \Gamma^{1} \neq \Gamma^{1}
$$

is not equal to 1 . The consequence is that in the high energy regime which is characteristic of the Hawking effect the full dynamics $\not D$ and the free dynamics $\mathbb{D}_{H}$ or $\mathbb{D}_{\mathfrak{s}}$ are no longer close to each other. Now recall that $\partial_{t} \widehat{r}=-1$ along incoming null geodesics with the correct sign of $\theta_{0}^{\prime}$. This means that the observable $\widehat{r}$ should increase (resp. decrease) exactly like $t$ along the evolution if we focus on scattering directions on which $\widehat{r}$ increases (decreases) in this way. We therefore choose

$$
\ell^{a}=\lambda N^{+, a}, \quad n^{a}=\lambda N^{-, a}
$$


for some normalization constant $\lambda$. The choice of $m^{a}$ is now imposed except for a factor of modulus 1 . We find

$$
\left\{\begin{aligned}
\ell^{a} & =\sqrt{\frac{\sigma^{2}}{2 \rho^{2} \Delta}}\left(\partial_{t}+\frac{\left(r^{2}+a^{2}\right)^{2}}{\sigma^{2}} k^{\prime}\left(r_{*}\right) \partial_{r_{*}}+\frac{\Delta}{\sigma^{2}} a \cos \theta \partial_{\theta}+\frac{a\left(2 M r-Q^{2}\right)}{\sigma^{2}} \partial_{\varphi}\right) \\
n^{a} & =\sqrt{\frac{\sigma^{2}}{2 \rho^{2} \Delta}}\left(\partial_{t}-\frac{\left(r^{2}+a^{2}\right)^{2}}{\sigma^{2}} k^{\prime}\left(r_{*}\right) \partial_{r_{*}}-\frac{\Delta}{\sigma^{2}} a \cos \theta \partial_{\theta}+\frac{a\left(2 M r-Q^{2}\right)}{\sigma^{2}} \partial_{\varphi}\right) \\
m^{a} & =\sqrt{\frac{\rho^{2}}{2 \sigma^{2}}}\left(i \frac{r^{2}+a^{2}}{\rho^{2}}\left(a \cos \theta \partial_{r_{*}}-k^{\prime}\left(r_{*}\right) \partial_{\theta}\right)+\frac{1}{\sin \theta} \partial_{\varphi}\right)
\end{aligned}\right.
$$

Note that in the $(t, \widehat{r}, \omega)$ coordinate system we have

$$
\begin{aligned}
\ell^{a} & =\sqrt{\frac{\sigma^{2}}{2 \rho^{2} \Delta}}\left(\partial_{t}+\partial_{\widehat{r}}+\frac{\Delta}{\sigma^{2}} a \cos \theta \partial_{\theta}+\frac{a\left(2 M r-Q^{2}\right)}{\sigma^{2}} \partial_{\varphi}\right), \\
n^{a} & =\sqrt{\frac{\sigma^{2}}{2 \rho^{2} \Delta}}\left(\partial_{t}-\partial_{\widehat{r}}-\frac{\Delta}{\sigma^{2}} a \cos \theta \partial_{\theta}+\frac{a\left(2 M r-Q^{2}\right)}{\sigma^{2}} \partial_{\varphi}\right), \\
m^{a} & =\sqrt{\frac{\rho^{2}}{2 \sigma^{2}}}\left(-i k^{\prime} \frac{r^{2}+a^{2}}{\rho^{2}} \partial_{\theta}+\frac{1}{\sin \theta} \partial_{\varphi}\right)
\end{aligned}
$$

and that the tetrad $\ell^{a}, n^{a}, m^{a}$ is adapted to the foliation.

4.4.2. The new expression of the Dirac equation. - Let us put

$$
\begin{aligned}
& e_{1}^{a}=L^{a}, \quad e_{2}^{a}=N^{a}, \quad e_{3}^{a}=M^{a}, \quad e_{4}^{a}=\bar{M}^{a}, \\
& \mathrm{e}_{a}^{\mathbf{1}}=L_{a}, \quad \mathrm{e}_{a}^{\mathbf{2}}=N_{a}, \quad \mathrm{e}_{a}^{\mathbf{3}}=M_{a}, \quad \mathrm{e}_{a}^{\mathbf{4}}=\bar{M}_{a} .
\end{aligned}
$$

In order to find the new expression of the Dirac equation we express $\ell^{a}, n^{a}, m^{a}, \bar{m}^{a}$ in terms of $L^{a}, N^{a}, M^{a}, \bar{M}^{a}$ and find the Lorentz transformation

$$
L_{\boldsymbol{a}}^{\boldsymbol{b}}=L_{a}^{b} e_{\boldsymbol{a}}^{a} e_{b}^{\boldsymbol{b}}=\frac{1}{2}\left(\begin{array}{cccc}
1+\alpha & 1-\alpha & \beta & \beta \\
1-\alpha & 1+\alpha & -\beta & -\beta \\
i \beta & -i \beta & -i(1+\alpha) & i(1-\alpha) \\
-i \beta & i \beta & -i(1-\alpha) & i(1+\alpha)
\end{array}\right),
$$

where $\alpha=k^{\prime} h^{2}$ and $\beta=\sqrt{\Delta / \sigma^{2}} a \cos \theta$, in particular

$$
\alpha^{2}+\beta^{2}=1 \text {. }
$$

We associate to the Newman-Penrose tetrads $L^{a}, N^{a}, M^{a}, \bar{M}^{a}$ (resp. $\ell^{a}, n^{a}, m^{a}, \bar{m}^{a}$ ) the spin frames $\left\{\tilde{\epsilon}_{0}^{A}, \tilde{\epsilon}_{1}^{A}\right\}$ (resp. $\left\{\epsilon_{0}^{A}, \epsilon_{1}^{A}\right\}$ ) as explained in Section 4.3. The matrix $S_{\boldsymbol{A}}^{\boldsymbol{B}}$ of the corresponding spin transformation $S_{A}^{B}$ in the spin frame $\left\{\tilde{\epsilon}_{0}^{A}, \tilde{\epsilon}_{1}^{A}\right\}$ is uniquely determined, modulo sign, by

$$
L_{a}^{b}=S_{A}^{B} \bar{S}_{A^{\prime}}^{B^{\prime}} \quad \text { and } \quad \operatorname{det}\left(S_{\boldsymbol{A}}^{\boldsymbol{B}}\right)=1 .
$$


The first condition can be expressed in terms of coordinates as

$$
L_{\boldsymbol{a}}^{\boldsymbol{b}}=\left(\begin{array}{cccc}
\left|S_{0}^{0}\right|^{2} & \left|S_{0}^{1}\right|^{2} & S_{0}^{0} \bar{S}_{0^{\prime}}^{1^{\prime}} & S_{0}^{1} \bar{S}_{0^{\prime}}^{0^{\prime}} \\
\left|S_{1}^{0}\right|^{2} & \left|S_{1}^{1}\right|^{2} & S_{1}^{0} \bar{S}_{1^{\prime}}^{1^{\prime}} & S_{1}^{1} \bar{S}_{1^{\prime}}^{0^{\prime}} \\
S_{0}^{0} \bar{S}_{1^{\prime}}^{0^{\prime}} & S_{0}^{1} \bar{S}_{1^{\prime}}^{1^{\prime}} & S_{0}^{0} \bar{S}_{1^{\prime}}^{1^{\prime}} & S_{0}^{1} \bar{S}_{1^{\prime}}^{0^{\prime}} \\
S_{1}^{0} \bar{S}_{0^{\prime}}^{0^{\prime}} & S_{1}^{1} \bar{S}_{0^{\prime}}^{1^{\prime}} & S_{1}^{0} \bar{S}_{0^{\prime}}^{1^{\prime}} & S_{1}^{1} \bar{S}_{0^{\prime}}^{0^{\prime}}
\end{array}\right) .
$$

We find

$$
S_{\boldsymbol{A}}^{\boldsymbol{B}}=\left(\begin{array}{cc}
S_{0}^{0} & S_{0}^{1} \\
S_{1}^{0} & S_{1}^{1}
\end{array}\right)=\frac{1}{\sqrt{2(1+\alpha)}} \mathrm{e}^{-\frac{1}{4} i \pi}\left(\begin{array}{cc}
1+\alpha & \beta \\
-i \beta & i(1+\alpha)
\end{array}\right)=: U .
$$

Let $\phi_{\boldsymbol{C}}$ (resp. $\widetilde{\phi}_{\boldsymbol{C}}$ ) be the components of $\phi_{A}$ in the spin frame $\left\{\epsilon_{0}^{A}, \epsilon_{1}^{A}\right\}$ (resp. $\left\{\tilde{\epsilon}_{0}^{A}, \tilde{\epsilon}_{1}^{A}\right\}$ ) and $\chi^{C^{\prime}}$ (resp. $\left.\widetilde{\chi}^{\boldsymbol{C}^{\prime}}\right)$ the components of $\chi^{A^{\prime}}$ in the spin frames $\left\{\epsilon_{0^{\prime}}^{A^{\prime}}, \epsilon_{1^{\prime}}^{A^{\prime}}\right\}$ (resp. $\left.\left\{\tilde{\epsilon}_{0^{\prime}}^{A^{\prime}}, \tilde{\epsilon}_{1^{\prime}}^{A^{\prime}}\right\}\right)$. We have

$$
\begin{aligned}
& \phi_{\boldsymbol{C}}=\phi_{A} \epsilon_{\boldsymbol{C}}^{A}=\phi_{A} S_{B}^{A} \tilde{\epsilon}_{\boldsymbol{C}}^{B}=\widetilde{\phi}_{\boldsymbol{A}} \tilde{\epsilon}_{A}^{\boldsymbol{A}} S_{B}^{A} \tilde{\epsilon}_{\boldsymbol{C}}^{B}=\tilde{\phi}_{\boldsymbol{A}} S_{\boldsymbol{C}}^{\boldsymbol{A}},
\end{aligned}
$$

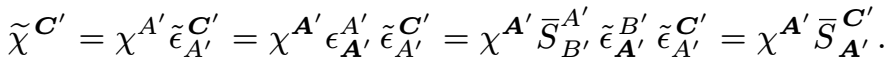

Noting that the matrix $\bar{S}_{\boldsymbol{A}^{\prime}}^{\boldsymbol{C}^{\prime}}$ is the inverse of $S_{\boldsymbol{C}}^{\boldsymbol{A}}$ it follows ${ }^{(1)}$

$$
\chi^{\boldsymbol{C}^{\prime}}=\sum_{\boldsymbol{A}=0}^{1} S_{\boldsymbol{C}}^{\boldsymbol{A}} \widetilde{\chi}^{\boldsymbol{A}^{\prime}}
$$

We put

$$
\mathcal{U}:=\left(\begin{array}{ll}
U & 0 \\
0 & U
\end{array}\right)
$$

Our aim is to calculate

$$
\widehat{D}:=U \not D \mathcal{U}^{*}
$$

We define

$$
\widehat{\Gamma}^{j}:=\mathcal{U} \Gamma^{j} \mathcal{U}^{*}, \quad 1 \leq j \leq 4,
$$

and find

$$
\begin{gathered}
\widehat{\Gamma}^{j}=\left(\begin{array}{cc}
\widehat{\sigma}^{j} & 0 \\
0 & -\widehat{\sigma}^{j}
\end{array}\right), \quad 1 \leq j \leq 3, \quad \widehat{\Gamma}^{4}=\Gamma^{4}, \\
\widehat{\sigma}^{1}:=\left(\begin{array}{cc}
\alpha & i \beta \\
-i \beta & -\alpha
\end{array}\right), \quad \widehat{\sigma}^{2}:=\left(\begin{array}{cc}
\beta & -i \alpha \\
i \alpha & -\beta
\end{array}\right), \quad \widehat{\sigma}^{3}:=\left(\begin{array}{cc}
0 & -1 \\
-1 & 0
\end{array}\right) .
\end{gathered}
$$

(1) We make the usual convention that $\boldsymbol{A}=\boldsymbol{A}^{\prime}$ numerically. 
The Dirac operator $\widehat{D}$ can be written in the form

$$
\begin{aligned}
& \widehat{D}=\left.\begin{array}{cc}
M_{r_{*}} & 0 \\
0 & -M_{r_{*}}
\end{array}\right)+\left(\begin{array}{cc}
M_{\theta} & 0 \\
0 & -M_{\theta}
\end{array}\right)+\frac{a_{0} h^{2}}{\sin \theta} \widehat{\Gamma}^{3} D_{\varphi} \\
& \quad+h^{2} c_{1}+h^{2} c_{2}^{\varphi} D_{\varphi}+\widehat{V}_{\varphi} D_{\varphi}+\widehat{V}_{1}, \\
& \widehat{V}_{\varphi}=-\frac{\sqrt{\Delta}}{\sigma \sin \theta}\left(\frac{\rho^{2}}{\sigma}-1\right) \widehat{\Gamma}^{3}-\frac{a\left(2 M r-Q^{2}\right)}{\left(r^{2}+a^{2}\right) \sigma^{2}}\left(r^{2}+a^{2}-\sigma\right),
\end{aligned}
$$




$$
\begin{aligned}
& \widehat{V}_{1}=\widehat{\mathbb{V}}_{0}+\frac{m \sqrt{\Delta}}{\sigma}\left(\rho-\sqrt{r^{2}+a^{2}}\right) \widehat{\Gamma}^{4}-\frac{q Q r}{\sigma^{2}}\left(r^{2}+a^{2}-\sigma\right), \\
& \widehat{\mathbb{V}}_{0}=\left(\begin{array}{cc}
\widehat{\mathbb{V}}_{1} & 0 \\
0 & \widehat{\mathbb{V}}_{1}
\end{array}\right), \quad \widehat{\mathbb{V}}_{1}=U\left(\begin{array}{cc}
v_{0} & v_{1} \\
\bar{v}_{1} & -v_{0}
\end{array}\right) U^{*}, \\
& M_{r_{*}}=\frac{1}{2}\left(\begin{array}{cc}
m_{r_{*}}^{1} & i m_{r_{*}}^{2} \\
-i m_{r_{*}}^{2} & -m_{r_{*}}^{1}
\end{array}\right), \\
& m_{r_{*}}^{1}=\sqrt{\alpha+1} h D_{r_{*}} h \sqrt{\alpha+1}-\frac{\beta h}{\sqrt{\alpha+1}} D_{r_{*}} \frac{\beta h}{\sqrt{\alpha+1}}, \\
& m_{r_{*}}^{2}=\sqrt{\alpha+1} h D_{r_{*}} \frac{\beta h}{\sqrt{\alpha+1}}+\frac{\beta h}{\sqrt{\alpha+1}} D_{r_{*}} h \sqrt{\alpha+1}, \\
& M_{\theta}=\frac{1}{2}\left(\begin{array}{cc}
m_{\theta}^{1} & i m_{\theta}^{2} \\
-i m_{\theta}^{2} & -m_{\theta}^{1}
\end{array}\right), \\
& m_{\theta}^{1}=\frac{\beta h \sqrt{a_{0}}}{\sqrt{\alpha+1}}\left(D_{\theta}+\frac{\cot \theta}{2 i}\right) \sqrt{a_{0}} h \sqrt{\alpha+1}+\sqrt{\alpha+1} h \sqrt{a_{0}}\left(D_{\theta}+\frac{\cot \theta}{2 i}\right) \frac{\beta h \sqrt{a_{0}}}{\sqrt{\alpha+1}}, \\
& m_{\theta}^{2}=\frac{\beta h \sqrt{a_{0}}}{\sqrt{\alpha+1}}\left(D_{\theta}+\frac{\cot \theta}{2 i}\right) \frac{h \beta \sqrt{a_{0}}}{\sqrt{\alpha+1}}-\sqrt{\alpha+1} h \sqrt{a_{0}}\left(D_{\theta}+\frac{\cot \theta}{2 i}\right) \sqrt{a_{0}} h \sqrt{\alpha+1} .
\end{aligned}
$$

We now want to use the variable introduced in (3.38). Let

$$
\mathcal{H}:=L^{2}\left(\left(\mathbb{R} \times S^{2}, \mathrm{~d} \widehat{r} \mathrm{~d} \omega\right) ; \mathbb{C}^{4}\right) .
$$

We define

$$
V: \mathscr{H}_{*} \longrightarrow \mathcal{H}, \quad u\left(r_{*}, \omega\right) \longmapsto u\left(r_{*}(\widehat{r}, \theta), \omega\right) k^{\prime-\frac{1}{2}}\left(r_{*}(\widehat{r}, \theta)\right)
$$

The map $V$ is a unitary transformation whose inverse is

$$
V^{*}: \mathcal{H} \longrightarrow \mathcal{H}_{*}, \quad v(\widehat{r}, \omega) \longmapsto v\left(\widehat{r}\left(r_{*}, \theta\right), \omega\right) k^{\prime \frac{1}{2}}\left(r_{*}\right) .
$$

The hamiltonian we want to work with is

$$
H:=V \widehat{\mathbb{D}} V^{*},
$$

which acts on $\mathcal{H}$. The operator $H$ is selfadjoint with domain

$$
D(H)=\left\{u \in \mathcal{H} ; \mathcal{U}^{*} V^{*} u \in D(\not D)\right\}
$$

and its spectrum is purely absolutely continuous. This follows from the corresponding results for $\not D$. In order to calculate $H$ we first observe that

$$
V D_{r_{*}} V^{*}=\left(k^{\prime}\right)^{\frac{1}{2}} D_{\widehat{r}}\left(k^{\prime}\right)^{\frac{1}{2}}, \quad V D_{\theta} V^{*}=a \cos \theta D_{\widehat{r}}+D_{\theta} .
$$


Now observe that

$$
\begin{aligned}
\frac{1}{2}\left(h^{2}(\alpha+1) k^{\prime}-\frac{\beta^{2} h^{2}}{(\alpha+1)} k^{\prime}+2 \beta h^{2} a_{0} a \cos \theta\right) & =\alpha h^{2} k^{\prime}+\beta h^{2} a_{0} a \cos \theta \\
& =\alpha^{2}+\beta^{2}=1, \\
i\left(2 h^{2} \beta k^{\prime}+\frac{\beta^{2} h^{2}}{\alpha+1} a_{0} a \cos \theta-(\alpha+1) h^{2} a_{0} a \cos \theta\right) & =i(2 \alpha \beta+(1-\alpha) \beta-(1+\alpha) \beta) \\
& =0
\end{aligned}
$$

and that

$$
\begin{gathered}
\sqrt{\alpha+1} h\left(k^{\prime}\right)^{\frac{1}{2}} \partial_{\widehat{r}}\left(\left(k^{\prime}\right)^{\frac{1}{2}} h \sqrt{\alpha+1}\right)-\frac{\beta h}{\sqrt{\alpha+1}}\left(k^{\prime}\right)^{\frac{1}{2}} \partial_{\widehat{r}}\left(\frac{\beta h}{\sqrt{\alpha+1}}\left(k^{\prime}\right)^{\frac{1}{2}}\right) \\
+\frac{\beta h \sqrt{a_{0}}}{\sqrt{\alpha+1}} a \cos \theta \partial_{\widehat{r}}\left(h \sqrt{a_{0}} \sqrt{\alpha+1}\right)+\sqrt{\alpha+1} h \sqrt{a_{0}} a \cos \theta \partial_{\widehat{r}}\left(\frac{\sqrt{a_{0}} \beta h}{\sqrt{\alpha+1}}\right) \\
=\frac{1}{2} \partial_{\widehat{r}}\left(k^{\prime} h^{2}(\alpha+1)\right)-\frac{1}{2} \partial_{\widehat{r}}\left(k^{\prime} \frac{\beta^{2} h^{2}}{\alpha+1}\right)+\partial_{\widehat{r}}\left(\beta h^{2} a_{0} a \cos \theta\right)=\partial_{\widehat{r}}\left(\alpha^{2}+\beta^{2}\right)=0, \\
\sqrt{\alpha+1} h\left(k^{\prime}\right)^{\frac{1}{2}} \partial_{\widehat{r}}\left(\left(k^{\prime}\right)^{\frac{1}{2}} \frac{\beta h}{\sqrt{\alpha+1}}\right)+\frac{\beta h}{\sqrt{\alpha+1}}\left(k^{\prime}\right)^{\frac{1}{2}} \partial_{\widehat{r}}\left(\left(k^{\prime}\right)^{\frac{1}{2}} h \sqrt{\alpha+1}\right) \\
+\frac{\beta h \sqrt{a_{0}}}{\sqrt{\alpha+1}} \partial_{\widehat{r}}\left(a \cos \theta \frac{\sqrt{a_{0}} h \beta}{\sqrt{\alpha+1}}\right)-\sqrt{\alpha+1} h \sqrt{a_{0}} \partial_{\widehat{r}}\left(a \cos \theta \sqrt{a_{0}} h \sqrt{\alpha+1}\right) \\
=\partial_{\widehat{r}}(\alpha \beta)+\frac{1}{2} \partial_{\widehat{r}}\left(\frac{\beta^{3}}{\alpha+1}-\beta(\alpha+1)\right)=0 .
\end{gathered}
$$

Therefore we obtain

$$
\begin{aligned}
& H=\Gamma^{1} D_{\widehat{r}}+\left(\begin{array}{cc}
M_{\theta} & 0 \\
0 & -M_{\theta}
\end{array}\right)+h^{2} a_{0} \widehat{\Gamma}^{3} \frac{D_{\varphi}}{\sin \theta}+h^{2} c_{1}+h^{2} c_{2}^{\varphi} D_{\varphi}+\widehat{V}_{\varphi} D_{\varphi}+\widehat{V}_{1} \\
& =\Gamma^{1} D_{\widehat{r}}+\left(m_{\theta}^{1} \Gamma^{1}-m_{\theta}^{2} \Gamma^{3}\right)-h^{2} a_{0} \Gamma^{2} \frac{D_{\varphi}}{\sin \theta}+h^{2} c_{1}+h^{2} c_{2}^{\varphi} D_{\varphi}+\widehat{V}_{\varphi} D_{\varphi}+\widehat{V}_{1} .
\end{aligned}
$$

Recalling that

$$
P_{\omega}:=\left(\begin{array}{cc}
M_{\theta} & 0 \\
0 & -M_{\theta}
\end{array}\right)+\widehat{\Gamma}^{3} h^{2} a_{0} \frac{D_{\varphi}}{\sin \theta}=U h \sqrt{a_{0}} \not_{S^{2}} \sqrt{a_{0}} h \mathcal{U}^{*},
$$

where all functions have to be evaluated at $\left(r_{*}(\widehat{r}, \theta), \theta\right)$, we see that the angular part is regular. Let us also define

$$
W:=H-\Gamma^{1} D_{\widehat{r}}-P_{\omega} .
$$

We put

$$
\mathcal{H}^{1}:=D(H), \quad\|u\|_{\mathcal{H}^{1}}^{2}=\|H u\|^{2}+\|u\|^{2} .
$$


Lemma 4.1. - (i) For all $u \in D(\not D)$,

$$
\begin{aligned}
& \left\|D_{r_{*}} u\right\|_{\mathcal{H}_{*}} \lesssim\|\not D u\|_{\mathcal{H}_{*}}+\|u\|_{\mathcal{H}_{*}}, \\
& \left\|a_{0} D_{S^{2}} u\right\|_{\mathcal{H}_{*}} \lesssim\|\not D u\|_{\mathcal{H}_{*}}+\|u\|_{\mathcal{H}_{*}} .
\end{aligned}
$$

(ii) For all $v \in D(H)$,

$$
\begin{aligned}
& \left\|D_{\widehat{r}} v\right\|_{\mathscr{H}} \lesssim\|H v\|_{\mathscr{H}}+\|v\|_{\mathscr{H}}, \\
& \left\|a_{0} D_{S^{2}} v\right\|_{\mathscr{H}} \lesssim\|H v\|_{\mathscr{H}}+\|v\|_{\mathscr{H}} .
\end{aligned}
$$

Proof. - For part $(i)$ see [15]. Let us show (ii). From (4.41) we infer for $v \in D(H)$ :

$$
-\left\langle\vee \mathcal{U} \partial_{r_{*}}^{2} \mathcal{u}^{*} v^{*} v, v\right\rangle \lesssim(\|H v\|+\|v\|)^{2}
$$

But we have

$$
\begin{aligned}
-V \mathcal{U} \partial_{r_{*}}^{2} \mathcal{U}^{*} V^{*}= & -\left[\mathcal{U}\left(k^{\prime}\right)^{\frac{1}{2}}, \partial_{\widehat{r}}\right] k^{\prime} \partial_{\widehat{r}}\left(k^{\prime}\right)^{\frac{1}{2}} \mathcal{U}^{*} \\
& \quad-\partial_{\widehat{r}} \mathcal{U}\left(k^{\prime}\right)^{\frac{3}{2}}\left[\partial_{\widehat{r}},\left(k^{\prime}\right)^{\frac{1}{2}} \mathcal{U}^{*}\right]-\partial_{\widehat{r}}\left(k^{\prime}\right)^{2} \partial_{\widehat{r}} \\
\geq & (\epsilon-\delta) \partial_{\widehat{r}}^{2}-C_{\epsilon}
\end{aligned}
$$

with $\delta=\min \left(k^{\prime 2}\right)>0$. This gives (4.43). Inequality (4.44) can be established in a similar way. We use

$$
V D_{\theta}^{2} V^{*}=\ell^{\prime 2} D_{\widehat{r}}^{2}+2 \ell^{\prime} D_{\widehat{r}} D_{\theta}+D_{\theta}^{2}
$$

as well as (4.43).

REMARK 4.2. - A precise analysis of the constants in [15] shows that

$$
\begin{gathered}
\forall \epsilon>0, \exists C_{\epsilon}>0, \forall u \in D(\not D), \quad\left\|D_{r_{*}} u\right\|_{\mathscr{H}_{*}} \leq(1+\epsilon)\|\not D u\|_{\mathcal{H}_{*}}+C_{\epsilon}\|u\|_{\mathcal{H}_{*}}, \\
\forall \epsilon>0, \exists C_{\epsilon}>0, \forall u \in D(H), \quad\left\|D_{\widehat{r}} u\right\|_{\mathcal{H}} \leq(1+\epsilon)\|H u\|_{\mathcal{H}}+C_{\epsilon}\|u\|_{\mathscr{H}^{*}} .
\end{gathered}
$$

4.4.3. Scattering results. - A complete scattering theory for massless Dirac fields in the Kerr metric was obtained in [29]. This result has been generalized to the case of massive charged Dirac fields in [15]. In both works the comparison dynamics are the dynamics which are natural in the $\left(t, r_{*}, \omega\right)$ coordinate system and the $L^{a}, N^{a}, M^{a}$ Newman-Penrose tetrad. We will need comparison dynamics which are natural with respect to the $(t, \widehat{r}, \theta, \varphi)$ coordinate system and the $\ell^{a}, n^{a}, m^{a}$ tetrad. To this purpose we define

$$
\begin{aligned}
& H_{\leftarrow}=\Gamma^{1} D_{\widehat{r}}-\frac{a}{r_{+}^{2}+a^{2}} D_{\varphi}-\frac{q Q r_{+}}{r_{+}^{2}+a^{2}}, \\
& H_{\rightarrow}=V \mathcal{U} D_{\rightarrow} \mathcal{U}^{*} V^{*},
\end{aligned}
$$

where $D_{\rightarrow}$ is defined in (4.29). These operators are selfadjoint on $\mathcal{H}$ with domains

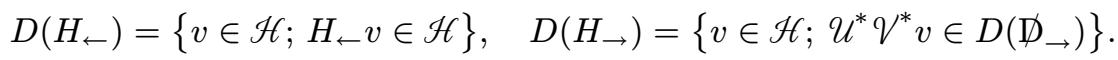


Let

$$
\begin{aligned}
& \mathcal{H}^{+}=\left\{v=\left(v_{1}, v_{2}, v_{3}, v_{4}\right) \in \mathcal{H} ; v_{1}=v_{4}=0\right\} \\
& \mathcal{H}^{-}=\left\{v=\left(v_{1}, v_{2}, v_{3}, v_{4}\right) \in \mathcal{H} ; v_{2}=v_{3}=0\right\}
\end{aligned}
$$

We note that

$$
\mathbf{1}_{\mathbb{R}^{ \pm}}\left(-\Gamma^{1}\right)=P_{\mathscr{H}^{ \pm}},
$$

where $P_{\mathscr{H}^{ \pm}}$is the projection from $\mathscr{H}$ to $\mathscr{H}^{ \pm}$. We also define the projections

$$
\begin{array}{cc}
P_{2,3}: \mathscr{H} \longrightarrow\left(L^{2}\left(\mathbb{R} \times S^{2}\right)\right)^{2}, & \left(v_{1}, v_{2}, v_{3}, v_{4}\right) \longmapsto\left(v_{2}, v_{3}\right), \\
P_{1,4}: \mathscr{H} \longrightarrow\left(L^{2}\left(\mathbb{R} \times S^{2}\right)\right)^{2}, & \left(v_{1}, v_{2}, v_{3}, v_{4}\right) \longmapsto\left(v_{1}, v_{4}\right) .
\end{array}
$$

Let

$$
\mathfrak{v}:=D_{r_{*}} \not D_{\rightarrow}^{-1}, \quad \widehat{\mathfrak{v}}=\vee \mathcal{u} \mathfrak{v} \mathcal{U}^{*} v^{*}
$$

be the "classical velocity operators" associated to $D_{\rightarrow}$ and $H_{\rightarrow}$. The following proposition gives the existence of the asymptotic velocity:

Proposition 4.4. - There exist selfadjoint operators $P^{ \pm}$such that for all $g$ in $C_{\infty}(\mathbb{R})$ we have

$$
g\left(P^{ \pm}\right)=s-\lim _{t \rightarrow \pm \infty} \mathrm{e}^{-i t H} g\left(\frac{\widehat{r}}{t}\right) \mathrm{e}^{i t H} .
$$

The operators $P^{ \pm}$commute with $H$. Furthermore we have

$$
\begin{aligned}
g\left(P^{ \pm}\right) \mathbf{1}_{\mathbb{R}^{ \pm}}\left(P^{ \pm}\right) & =s-\lim _{t \rightarrow \pm \infty} \mathrm{e}^{-i t H} g(\hat{\mathfrak{v}}) \mathrm{e}^{i t H} \mathbf{1}_{\mathbb{R}^{ \pm}}\left(P^{ \pm}\right), \\
g\left(P^{ \pm}\right) \mathbf{1}_{\mathbb{R}^{\mp}}\left(P^{ \pm}\right) & =s-\lim _{t \rightarrow \pm \infty} \mathrm{e}^{-i t H} g\left(-\Gamma^{1}\right) \mathrm{e}^{i t H} \mathbf{1}_{\mathbb{R}^{\mp}}\left(P^{ \pm}\right), \\
\sigma\left(P^{ \pm}\right) & =\{-1\} \cup[0,1] \\
\sigma_{p p}(H) & =\mathbf{1}_{\{0\}}\left(P^{ \pm}\right)=\varnothing .
\end{aligned}
$$

REMARK 4.3. - a) For limits of the form (4.46) we will write

$$
P^{ \pm}=s-C_{\infty}-\lim _{t \rightarrow \pm \infty} \mathrm{e}^{-i t H} \frac{\widehat{r}}{t} \mathrm{e}^{i t H}
$$

b) We can construct in the same way

$$
P_{\rightarrow}^{ \pm}=s-C_{\infty}-\lim _{t \rightarrow \pm \infty} \mathrm{e}^{-i t H_{\rightarrow}} \frac{\widehat{r}}{t} \mathrm{e}^{i t H_{\rightarrow}} .
$$

Proof. - Using the definition of $H$ we see that it is sufficient to show the existence of

$$
s-\lim _{t \rightarrow \pm \infty} \mathrm{e}^{-i t \not D} g\left(\frac{\widehat{r}\left(r_{*}, \theta\right)}{t}\right) \mathrm{e}^{i t \not D} .
$$

By [15, Theorem 5.2] we know that

$$
g\left(\widetilde{P}^{ \pm}\right)=s-\lim _{t \rightarrow \pm \infty} \mathrm{e}^{-i t \not D} g\left(\frac{r_{*}}{t}\right) \mathrm{e}^{i t \not D} \quad \text { exists. }
$$


As $g$ is uniformly continuous and $\left|\widehat{r}\left(r_{*}, \theta\right)-r_{*}\right| \lesssim 1$ we have

$$
s-\lim _{t \rightarrow \pm \infty} \mathrm{e}^{-i t \not D}\left(g\left(\frac{\widehat{r}\left(r_{*}, \theta\right)}{t}\right)-g\left(\frac{r_{*}}{t}\right)\right) \mathrm{e}^{i t \not D}=0 .
$$

This gives the existence. Equalities (4.47), (4.49), (4.50) follow directly from the definitions and the corresponding equalities in [15], so does (4.48) if we replace $\Gamma^{1}$ by $\widehat{\Gamma}^{1}$. In order to replace $\widehat{\Gamma}^{1}$ again by $\Gamma^{1}$ it is sufficient by a density argument to show

$$
\begin{aligned}
\forall \epsilon>0, \forall J \in C_{\infty}(\mathbb{R}), \operatorname{supp} J \subset(-\infty,-\epsilon) \\
\qquad-\lim _{t \rightarrow \infty} \mathrm{e}^{-i t \not D}\left(g\left(-\widehat{\Gamma}^{1}\right)-g\left(-\Gamma^{1}\right)\right) J\left(\frac{r_{*}}{t}\right) \mathrm{e}^{i t \not D}=0 .
\end{aligned}
$$

We then note that it is sufficient to show (4.52) for smooth $g$. Indeed as $g$ is uniformly continuous we can approximate it in $L^{\infty}$ norm by smooth functions. Equation (4.52) follows for smooth $g$ from the observation that

$$
\widehat{\Gamma}^{1}-\Gamma^{1}=\left(\Gamma_{i j}\right), \quad \Gamma_{i j}=\theta\left(\mathrm{e}^{\kappa_{+} r_{*}}\right), \quad r_{*} \rightarrow-\infty .
$$

At infinity we define the Dollard modification

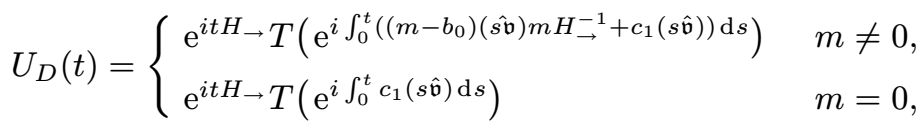

where $T$ denotes time ordering

$$
T\left(\mathrm{e}^{\int_{s}^{t} W(u) \mathrm{d} u}\right):=\sum_{n=0}^{\infty} \int_{t \geq u_{n} \geq \cdots \geq u_{1} \geq s} \cdots \int W\left(u_{n}\right) \cdots W\left(u_{1}\right) \mathrm{d} u_{n} \cdots \mathrm{d} u_{1}
$$

and $\hat{\mathfrak{v}}$ is the classical velocity operator (see $(4.45))$.

THEOREM 4.2. - The wave operators

$$
\begin{aligned}
& W_{\rightarrow}^{ \pm}=s-\lim _{t \rightarrow \pm \infty} \mathrm{e}^{-i t H} U_{D}(t) \mathbf{1}_{\mathbb{R}^{ \pm}}\left(P_{\rightarrow}^{ \pm}\right), \\
& \Omega_{\rightarrow}^{ \pm}=s-\lim _{t \rightarrow \pm \infty} U_{D}(-t) \mathrm{e}^{i t H} \mathbf{1}_{\mathbb{R}^{ \pm}}\left(P^{ \pm}\right), \\
& W_{\leftarrow}^{ \pm}=s-\lim _{t \rightarrow \pm \infty} \mathrm{e}^{-i t H} \mathrm{e}^{i t H \leftarrow P_{\mathcal{H}^{\mp}},} \\
& \Omega_{\leftarrow}^{ \pm}=s-\lim _{t \rightarrow \pm \infty} \mathrm{e}^{-i t H \leftarrow} \mathrm{e}^{i t H} \mathbf{1}_{\mathbb{R}^{\mp}}\left(P^{ \pm}\right)
\end{aligned}
$$

exist and satisfy

$$
\left(W_{\leftarrow}^{ \pm}\right)^{*}=\Omega_{\leftarrow}^{ \pm}, \quad\left(\Omega_{\leftarrow}^{ \pm}\right)^{*}=W_{\leftarrow}^{ \pm}, \quad\left(W_{\rightarrow}^{ \pm}\right)^{*}=\Omega_{\rightarrow}^{ \pm}, \quad\left(\Omega_{\rightarrow}^{ \pm}\right)^{*}=W_{\rightarrow}^{ \pm} .
$$

REMARK 4.4. - For the proof of the theorem about the Hawking effect we only need the asymptotic completeness result near the horizon. 
Proof. - Let

$$
\mathbb{D}_{\leftarrow}:=\mathcal{U}^{*} V^{*} H_{\leftarrow} \vee \mathcal{U}, \quad \widetilde{P}_{ \pm}:=\mathcal{U}^{*} V^{*} P_{\mathcal{H}^{ \pm}} V \mathcal{U}
$$

It is sufficient to show that the following limits exist:

$$
\begin{aligned}
& \widetilde{W}_{\rightarrow}^{ \pm}=s-\lim _{t \rightarrow \pm \infty} \mathrm{e}^{-i t \not D} \widetilde{U}_{D}(t) \mathbf{1}_{\mathbb{R}^{ \pm}}\left(\widetilde{P}_{\rightarrow}^{ \pm}\right), \\
& \widetilde{\Omega}_{\rightarrow}^{ \pm}=s-\lim _{t \rightarrow \pm \infty} \widetilde{U}_{D}(-t) \mathrm{e}^{i t \not D} \mathbf{1}_{\mathbb{R}^{ \pm}}\left(\widetilde{P}^{ \pm}\right), \\
& \widetilde{W}_{\leftarrow}^{ \pm}=s-\lim _{t \rightarrow \pm \infty} \mathrm{e}^{-i t \not D} \mathrm{e}^{i t \not D \leftarrow} \widetilde{P}_{\mp}, \\
& \widetilde{\Omega}_{\leftarrow}^{ \pm}=s-\lim _{t \rightarrow \pm \infty} \mathrm{e}^{-i t \not D \leftarrow} \mathrm{e}^{i t \not D} \mathbf{1}_{\mathbb{R}^{\mp}}\left(\widetilde{P}^{ \pm}\right)
\end{aligned}
$$

with

$$
\begin{gathered}
\widetilde{U}_{D}(t)=\mathcal{U}^{*} V^{*} U_{D}(t) \mathcal{V}, \\
\widetilde{P}^{ \pm}=s-C_{\infty}-\lim _{t \rightarrow \pm \infty} \mathrm{e}^{-i t \not D} \frac{r_{*}}{t} \mathrm{e}^{i t \not D}, \\
\widetilde{P}_{\rightarrow}^{ \pm}=s-C_{\infty}-\lim _{t \rightarrow \pm \infty} \mathrm{e}^{-i t \not D_{\rightarrow}} \frac{r_{*}}{t} \mathrm{e}^{i t \not D_{\rightarrow}} .
\end{gathered}
$$

The existence of the first two limits is contained in [15, Theorem 5.5]. We have

$$
\not_{\leftarrow}=\left(\begin{array}{cc}
A & 0 \\
0 & -A
\end{array}\right)-\frac{a}{r_{+}^{2}+a^{2}} D_{\varphi}-\frac{q Q r_{+}}{r_{+}^{2}+a^{2}}
$$

with $A=\frac{1}{2}\left(\begin{array}{ll}a_{11} & a_{12} \\ a_{21} & a_{22}\end{array}\right)$

$$
\begin{aligned}
& a_{11}=\sqrt{\alpha+1}\left(k^{\prime}\right)^{-\frac{1}{2}} D_{r_{*}}\left(k^{\prime}\right)^{-\frac{1}{2}} \sqrt{\alpha+1} \\
&-\frac{\beta}{\sqrt{\alpha+1}}\left(k^{\prime}\right)^{-\frac{1}{2}} D_{r_{*}}\left(k^{\prime}\right)^{-\frac{1}{2}} \frac{\beta}{\sqrt{\alpha+1}}=-a_{22}, \\
& a_{12}=\sqrt{\alpha+1}\left(k^{\prime}\right)^{-\frac{1}{2}} D_{r_{*}}\left(k^{\prime}\right)^{-\frac{1}{2}} \frac{\beta}{\sqrt{\alpha+1}}+h c=a_{21} .
\end{aligned}
$$

By [15, Theorem 5.4] we know that the limits

$$
\widetilde{W}_{H}^{ \pm}=s-\lim _{t \rightarrow \pm \infty} \mathrm{e}^{-i t \not D} \mathrm{e}^{i t \emptyset_{H}} P_{\mathcal{H}_{*}^{\mp}}, \quad \widetilde{\Omega}_{H}^{ \pm}=s-\lim _{t \rightarrow \pm \infty} \mathrm{e}^{-i t \emptyset_{H}} \mathrm{e}^{i t \not D} \mathbf{1}_{\mathbb{R}^{\mp}}\left(\widetilde{P}^{ \pm}\right)
$$

exist. Here $\mathscr{H}_{*}^{\mp}$ denote

$$
\begin{aligned}
& \mathscr{H}_{*}^{+}=\left\{u=\left(u_{1}, u_{2}, u_{3}, u_{4}\right) ; u_{1}=u_{4}=0\right\}, \\
& \mathcal{H}_{*}^{-}=\left\{u=\left(u_{1}, u_{2}, u_{3}, u_{4}\right) ; u_{2}=u_{3}=0\right\} .
\end{aligned}
$$

It is therefore sufficient to show the existence of

$$
W_{c}^{ \pm}=s-\lim _{t \rightarrow \pm \infty} \mathrm{e}^{-i t \not D_{\leftarrow}} \mathrm{e}^{i t \not_{H}} P_{\mathscr{H}_{*}^{\mp}}, \quad \Omega_{c}^{ \pm}=s-\lim _{t \rightarrow \pm \infty} \mathrm{e}^{-i t \not_{H}} \mathrm{e}^{i t \not D_{\leftarrow}} \widetilde{P}_{\mp} .
$$

and that

$$
P_{\mathcal{H}_{*}^{\mp}} \Omega_{c}^{ \pm}=\Omega_{c}^{ \pm}
$$


The existence of the first limit follows from Cook's method and the fact that

$$
k^{\prime}=1+\theta\left(\mathrm{e}^{\kappa_{+} r_{*}}\right), \quad \alpha=1+\Theta\left(\mathrm{e}^{\kappa_{+} r_{*}}\right), \quad \beta=\theta\left(\mathrm{e}^{\kappa_{+} r_{*}}\right), \quad r_{*} \rightarrow-\infty .
$$

The existence of $\Omega_{c}^{ \pm}$follows from the existence of

$$
\widehat{\Omega}_{c}^{ \pm}=s-\lim _{t \rightarrow \pm \infty} \mathrm{e}^{-i t H_{H}} \mathrm{e}^{i t H_{\leftarrow}} P_{\mathscr{H}^{\mp}},
$$

where $H_{H}=\vee U D_{H} U^{*} V^{*}$. This allows us to apply Cook's method also in this case. We omit the details. It remains to check (4.63). We note that $\widetilde{P}_{\mp}=\mathbf{1}_{\mathbb{R} \mp}\left(\widetilde{P}_{\leftarrow}^{ \pm}\right)$, where $\widetilde{P}_{\leftarrow}^{ \pm}$is the asymptotic velocity associated to $D_{\leftarrow}$. This follows from the argument used in the proof of Proposition 4.4. Thus

$$
\Omega_{c}^{ \pm}=\Omega_{c}^{ \pm} \mathbf{1}_{\mathbb{R} \mp}\left(\widetilde{P}_{\leftarrow}^{ \pm}\right)=\mathbf{1}_{\mathbb{R} \mp}\left(\widetilde{P}_{H}^{ \pm}\right) \Omega_{c}^{ \pm}=P_{\mathcal{H}_{*}^{\mp}} \Omega_{c}^{ \pm},
$$

where $\widetilde{P}_{H}^{ \pm}=-\Gamma^{1}$ is the asymptotic velocity associated to $\mathbb{D}_{H}$.

\subsection{The Dirac equation on $\mathcal{M}_{\text {col }}$}

We want to impose a boundary condition on the surface of the star such that the evolution can be described by a unitary propagator. We will use the conserved current

$$
V_{a}=\phi_{A} \bar{\phi}_{A^{\prime}}+\bar{\chi}_{A} \chi_{A^{\prime}} \text {. }
$$

Integrating over the domain indicated in Figure $1^{(2)}$ and supposing that the field is 0 in a neighborhood of $i^{0}$ gives by Stokes' theorem

$$
\int_{\Sigma_{s}} V_{a} T^{a} \mathrm{~d} \sigma_{\Sigma_{s}}-\int_{\Sigma_{t}} V_{a} T^{a} \mathrm{~d} \sigma_{\Sigma_{t}}+\int_{\phi_{I}} V_{a} \mathcal{N}^{a} \mathrm{~d} \sigma_{\phi_{I}}=0,
$$

where $\mathcal{N}^{a}$ is the normal to the surface of the star. Therefore the necessary condition for charge conservation outside the collapsing body is

$$
V_{a} \mathcal{N}^{a}=0 \text { on } \varnothing \text {. }
$$

We will impose

$$
\mathcal{N}^{A A^{\prime}} \phi_{A}=\frac{1}{\sqrt{2}} \mathrm{e}^{-i \nu} \chi^{A^{\prime}} \text { and } \mathcal{N}^{A A^{\prime}} \chi_{A^{\prime}}=\frac{1}{\sqrt{2}} \mathrm{e}^{i \nu} \phi^{A} \text { on } \phi .
$$

Here $\nu$ is the so called chiral angle. We note that (4.65) implies (4.64):

$$
\begin{aligned}
\sqrt{2} \mathcal{N}^{a} V_{a} & =\sqrt{2}\left(\mathcal{N}^{A A^{\prime}} \phi_{A} \bar{\phi}_{A^{\prime}}+\mathcal{N}^{A A^{\prime}} \bar{\chi}_{A} \chi_{A^{\prime}}\right) \\
& =\mathrm{e}^{-i \nu} \chi^{A^{\prime}} \bar{\phi}_{A^{\prime}}+\mathrm{e}^{-i \nu} \bar{\phi}^{A^{\prime}} \chi_{A^{\prime}}=0 .
\end{aligned}
$$

From (4.65) we obtain

$$
\mathcal{N}_{B A^{\prime}} \mathcal{N}^{A A^{\prime}} \phi_{A}=-\frac{1}{2} \phi_{B}
$$

(2) The Penrose compactification of block $I$ shown in Figure 1 is performed in Appendix B. 


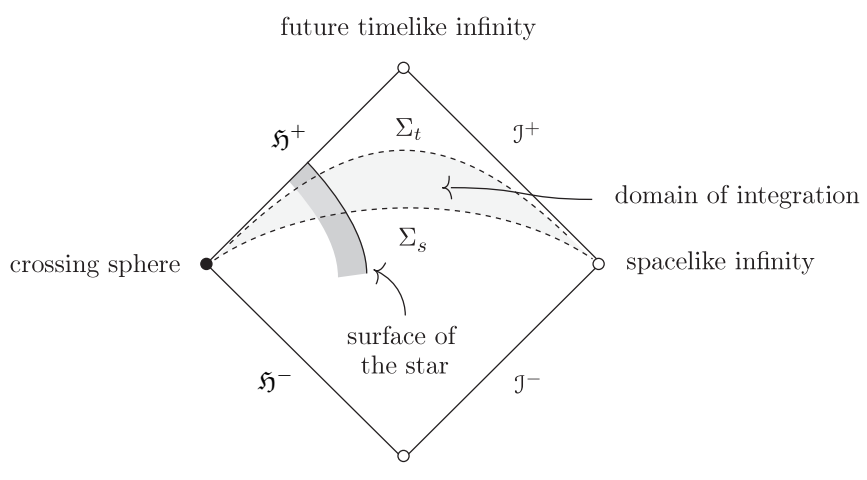

past timelike infinity

FiguRE 1. The surface of the star and the domain of integration.

We have

$$
\epsilon_{A C} \mathcal{N}_{B A^{\prime}} \mathcal{N}^{A A^{\prime}}=\mathcal{N}_{B A^{\prime}} \mathcal{N}_{C}^{A^{\prime}}=: \kappa_{B C}, \quad \kappa_{C B}=\mathcal{N}_{C A^{\prime}} \mathcal{N}_{B}^{A^{\prime}}=-\kappa_{B C} .
$$

From the antisymmetry of $\kappa_{B C}$ follows

$$
\kappa_{B C}=\frac{1}{2} \kappa_{A}^{A} \epsilon_{B C}=\frac{1}{2} \mathcal{N}^{a} \mathcal{N}_{a} \epsilon_{B C} .
$$

Thus (4.65) implies

$$
\mathcal{N}^{a} \mathcal{N}_{a} \phi_{B}=-\phi_{B}
$$

We therefore impose

$$
\mathcal{N}^{a} \mathcal{N}_{a}=-1 \text {. }
$$

This avoids that the boundary condition imposes $\left(\phi_{A}, \chi^{A^{\prime}}\right)=0$ on $\phi$. We will from now on suppose $\mathcal{N}_{a}$ to be past directed, but the opposite choice would of course be possible. Let us now rewrite condition (4.65) using a coordinate system and a spin frame. We have

$$
\mathcal{N}^{\boldsymbol{A} \boldsymbol{A}^{\prime}}=g^{\boldsymbol{a} \boldsymbol{A \boldsymbol { A } ^ { \prime }}} \mathcal{N}_{\boldsymbol{a}}={ }^{t} A^{\boldsymbol{a}} \mathcal{N}_{\boldsymbol{a}}, \quad \mathcal{N}_{\boldsymbol{A} \boldsymbol{A}^{\prime}}=g_{\boldsymbol{A} \boldsymbol{A}^{\prime}} \mathcal{N}_{\boldsymbol{a}}=B^{\boldsymbol{a}} \mathcal{N}_{\boldsymbol{a}}
$$

The boundary condition (4.65) implies

$$
\begin{aligned}
\sqrt{2}\left(\begin{array}{cc}
0 & B^{\boldsymbol{a}} \\
A^{\boldsymbol{a}} & 0
\end{array}\right) & \mathcal{N}_{\boldsymbol{a}}\left(\begin{array}{c}
\phi_{\boldsymbol{A}} \\
\chi^{\boldsymbol{A}^{\prime}}
\end{array}\right)=\left(\begin{array}{cc}
-\mathrm{e}^{i \nu} & 0 \\
0 & \mathrm{e}^{-i \nu}
\end{array}\right)\left(\begin{array}{c}
\phi_{\boldsymbol{A}} \\
\chi^{\boldsymbol{A}^{\prime}}
\end{array}\right) \\
& \Longleftrightarrow\left(\begin{array}{cc}
1 / i & 0 \\
0 & -1 / i
\end{array}\right) \gamma^{\boldsymbol{a}} \mathcal{N}_{\boldsymbol{a}}\left(\begin{array}{c}
\phi_{\boldsymbol{A}} \\
\chi^{\boldsymbol{A}^{\prime}}
\end{array}\right)=\left(\begin{array}{cc}
-\mathrm{e}^{i \nu} & 0 \\
0 & \mathrm{e}^{-i \nu}
\end{array}\right)\left(\begin{array}{c}
\phi_{\boldsymbol{A}} \\
\chi^{\boldsymbol{A}^{\prime}}
\end{array}\right) \\
& \Longleftrightarrow \gamma^{\boldsymbol{a}} \mathcal{N}_{\boldsymbol{a}}\left(\begin{array}{c}
\phi_{\boldsymbol{A}} \\
\chi^{\boldsymbol{A}^{\prime}}
\end{array}\right)=-i \mathcal{B}\left(\begin{array}{c}
\phi_{\boldsymbol{A}} \\
\chi^{\boldsymbol{A}^{\prime}}
\end{array}\right),
\end{aligned}
$$

where $\mathcal{B}=\mathrm{e}^{-i \nu \gamma^{5}}, \gamma^{5}=-i \gamma^{0} \gamma^{1} \gamma^{2} \gamma^{3}=\operatorname{Diag}(1,1,-1,-1), \nu \in \mathbb{R}$. The boundary condition (4.66) is usually called a MIT boundary condition. Using formula (4.11) we 
find $^{(3)}$

$$
\gamma^{t}=\kappa_{0} \gamma^{0}, \quad \gamma^{r_{*}}=\kappa_{0} h^{2} \gamma^{1}, \quad \gamma^{\theta}=\kappa_{0} a_{0} h^{2} \gamma^{2}, \quad \kappa_{0}=\frac{\sigma}{\rho \sqrt{\Delta}}
$$

In the $\left(t, r_{*}, \theta, \varphi\right)$ coordinate system and the $L^{a}, N^{a}, M^{a}, \bar{M}^{a}$ tetrad the boundary condition that we impose reads

$$
\sum_{\mu \in\left\{t, r_{*}, \theta, \varphi\right\}} \mathcal{N}_{\mu} \gamma^{\mu} \Phi=-i \mathscr{B} \Phi \text { on } \phi,
$$

where $\mathcal{N}_{\mu}$ are the coordinates of the conormal of the surface of the star in the $\left(t, r_{*}, \theta, \varphi\right)$ coordinate system. Let us now consider the $(t, \widehat{r}, \theta, \varphi)$ coordinate system and the $\ell^{a}, n^{a}, m^{a}, \bar{m}^{a}$ tetrad. We denote $\widehat{\gamma}^{t}, \widehat{\gamma}^{r}, \widehat{\gamma}^{\theta}, \widehat{\gamma}^{\varphi}$ the Dirac matrices with respect to these choices. We find

$$
\widehat{\gamma}^{t}=\kappa_{0} \gamma^{0}, \quad \widehat{\gamma}^{\widehat{r}}=\kappa_{0} \gamma^{1}, \quad \widehat{\gamma}^{\theta}=\kappa_{0} a_{0} h^{2} \widehat{\gamma}^{2}, \quad \widehat{\gamma}^{2}=i\left(\begin{array}{cc}
0 & \widehat{\sigma}^{2} \\
\widehat{\sigma}^{2} & 0
\end{array}\right) .
$$

Putting $\Psi=\mathcal{V} \mathcal{U} \Phi$ we find the following boundary condition for $\Psi$ :

$$
\sum_{\hat{\mu} \in\{t, \widehat{r}, \theta, \varphi\}} \mathcal{N}_{\hat{\mu}} \widehat{\gamma}^{\hat{\mu}} \Psi=-i \mathcal{B} \Psi \quad \text { on } \quad \varnothing .
$$

Here $\mathcal{N}_{\hat{\mu}}$ are the coordinates of the conormal in the $(t, \widehat{r}, \theta, \varphi)$ coordinate system. We will use in the following the $(t, \widehat{r}, \theta, \varphi)$ coordinate system.

We introduce the Hilbert spaces

$$
\mathcal{H}_{t}=\left(\left(L^{2}\left(\Sigma_{t}^{\mathrm{col}}, \mathrm{d} \widehat{r} \mathrm{~d} \omega\right)\right)^{4},\|\cdot\|_{t}\right),
$$

where the norm $\|\cdot\|_{t}$ is defined by

$$
\|\Psi\|_{t}=\left\|[\Psi]_{L}\right\|, \quad[\Psi]_{L}(\widehat{r}, \omega)= \begin{cases}\Psi(\widehat{r}, \omega) & \widehat{r} \geq \widehat{z}(t, \theta), \\ 0 & \widehat{r} \leq \widehat{z}(t, \theta) .\end{cases}
$$

Let

$$
\mathcal{H}_{t}^{1}=\left\{u \in \mathcal{H}_{t} ; H u \in \mathcal{H}_{t}\right\}, \quad\|u\|_{\mathcal{H}_{t}^{1}}^{2}=\|u\|_{t}^{2}+\|H u\|_{t}^{2} .
$$

We also need an extension from $\mathcal{H}_{t}^{1}$ to $\mathcal{H}^{1}$. To this purpose we put for $\phi \in \mathcal{H}_{t}^{1}$ :

$$
[\phi]_{H}(\widehat{r}, \omega)= \begin{cases}\phi(\widehat{r}, \omega) & \widehat{r} \geq \widehat{z}(t, \theta), \\ \phi(2 \widehat{z}(t, \theta)-\widehat{r}, \omega) & \widehat{r} \leq \widehat{z}(t, \theta) .\end{cases}
$$

It is easy to check that $[\phi]_{H}$ is in $\mathcal{H}^{1}$. The operator $\not_{t}$, the spaces $\mathcal{H}_{* t}, \mathcal{H}_{* t}^{1}$ as well as the extension [. $]_{H}^{*}$ are defined in an analogous way using the $\left(t, r_{*}, \theta, \varphi\right)$ coordinate

(3) As we will see we do not need the explicit form of $\gamma^{\varphi}, \widehat{\gamma}^{\varphi}$. 
system and the $\left(L^{a}, N^{a}, M^{a}\right)$ tetrad. On $\mathcal{M}_{\text {col }}$ we consider the mixed problem

$$
\left\{\begin{array}{l}
\partial_{t} \Psi=i H_{t} \Psi, \quad \widehat{z}(t, \theta)<\widehat{r}, \\
\left(\sum_{\hat{\mu} \in\{t, \widehat{r}, \theta, \varphi\}} \mathcal{N}_{\hat{\mu}} \widehat{\gamma}^{\hat{\mu}}\right) \Psi(t, \widehat{z}(t, \theta), \omega)=-i \mathcal{B} \Psi(t, \widehat{z}(t, \theta), \omega), \\
\Psi(t=s, .)=\Psi_{s}(.) .
\end{array}\right.
$$

Here the operator $H_{t}$ is given by $H_{t}=H$ and

$$
D\left(H_{t}\right)=\left\{\Psi \in \mathcal{H}_{t}^{1} ;\left(\sum_{\hat{\mu} \in\{t, \widehat{r}, \theta, \varphi\}} \mathcal{N}_{\hat{\mu}} \widehat{\gamma}^{\hat{\mu}} \Psi\right)(t, \widehat{z}(t, \theta), \omega)=-i \mathscr{B} \Psi(t, \widehat{z}(t, \theta), \omega)\right\} .
$$

REMARK 4.5 (Explicit calculation). - It will be helpful in the following to have a more explicit form of the boundary condition. We choose the $\left(t, r_{*}, \theta, \varphi\right)$ coordinate system and the $L^{a}, N^{a}, M^{a}, \bar{M}^{a}$ tetrad. The conormal of the surface of the star is

$$
\mathcal{N}_{a}=w(t, \theta) \rho a_{0} h^{2}\left(\dot{z} \mathrm{~d} t-\mathrm{d} r_{*}+\left(\partial_{\theta} z\right) \mathrm{d} \theta\right)
$$

and the boundary condition reads

$$
\begin{gathered}
w(t, \theta)\left(\begin{array}{cccc}
0 & 0 & \dot{z}-h^{2} & \left(\partial_{\theta} z\right) a_{0} h^{2} \\
0 & 0 & \left(\partial_{\theta} z\right) a_{0} h^{2} & \dot{z}+h^{2} \\
-\left(\dot{z}+h^{2}\right) & a_{0} h^{2}\left(\partial_{\theta} z\right) & 0 & 0 \\
a_{0} h^{2}\left(\partial_{\theta} z\right) & -\dot{z}+h^{2} & 0 & 0
\end{array}\right) \Phi=-\mathscr{B} \Phi \\
\Longleftrightarrow w(t, \theta) \Gamma^{4}\left(-\dot{z}-h^{2} \Gamma^{1}+\left(\partial_{\theta} z\right) a_{0} h^{2} \Gamma^{2}\right) \Phi=-i \mathscr{B} \Phi .
\end{gathered}
$$

Here $w(t, \theta)$ is a smooth function. We compute

$$
\mathcal{N}^{a}=\rho^{-1} a_{0}^{-1} h^{-2} w\left(\dot{z} \partial_{t}+h^{4} \partial_{r_{*}}+\dot{z} \frac{a\left(2 M r-Q^{2}\right)}{\sigma^{2}} \partial_{\varphi}-a_{0}^{2} h^{4}\left(\partial_{\theta} z\right) \partial_{\theta}\right) .
$$

Normalization $\mathcal{N}^{a} \mathcal{N}_{a}=-1$ gives

$$
w(t, \theta)=\left(h^{4}-\dot{z}^{2}+a_{0}^{2} h^{4}\left(\partial_{\theta} z\right)^{2}\right)^{-\frac{1}{2}} .
$$

In an analogous way we find in the $(t, \widehat{r}, \theta, \varphi)$ coordinate system and using the $\left(\ell^{a}, n^{a}, m^{a}, \bar{m}^{a}\right)$ tetrad

$$
\mathcal{N}_{a}=\widehat{w} \rho a_{0} h^{2}\left(\dot{\vec{z}} \mathrm{~d} t-\mathrm{d} \widehat{r}+\left(\partial_{\theta} \widehat{z}\right) \mathrm{d} \theta\right) .
$$

Therefore the boundary condition reads

$$
\widehat{w}(t, \theta) \widehat{\Gamma}^{4}\left(-\dot{\widehat{z}}-\Gamma^{1}+\left(\partial_{\theta} \widehat{z}\right) a_{0} h^{2} \widehat{\Gamma}^{2}\right) \Psi=-i \mathscr{B} \Psi .
$$

We compute

$$
\mathcal{N}^{a}=\rho^{-1} a_{0}^{-1} h^{-2} \widehat{w}\left\{\dot{\widehat{z}} \partial_{t}+\dot{\widehat{z}} \frac{a\left(2 M r-Q^{2}\right)}{\sigma^{2}} \partial_{\varphi}+\left(1-\left(\partial_{\theta} \widehat{z}\right) \frac{\Delta}{\sigma^{2}} \ell^{\prime}\right) \partial_{\widehat{r}}+\left(\ell^{\prime}-\left(\partial_{\theta} \widehat{z}\right)\right) \frac{\Delta}{\sigma^{2}} \partial_{\theta}\right\} .
$$

Normalization gives

$$
\widehat{w}(t, \theta)=\left(h^{4} k^{2}-\dot{\widehat{z}}^{2}+a_{0}^{2} h^{4}\left(\left(\partial_{\theta} \widehat{z}\right)-\ell^{\prime}\right)^{2}\right)^{-\frac{1}{2}} .
$$


Note that by assumption $(3.53) h^{4} k^{2}>\dot{\widehat{z}}^{2}$ and thus $h^{4}>\dot{z}^{2}$. We also note that by the previous considerations we have

$$
\operatorname{Rank}\left(-\dot{\widehat{z}}-\Gamma^{1}+\left(\partial_{\theta} \widehat{z}\right) a_{0} h^{2} \widehat{\Gamma}^{2}+\frac{i}{\widehat{w}} \widehat{\Gamma}^{4} \mathscr{B}\right)=2
$$

Let $\check{D}_{t}=\not_{t}+\dot{z} D_{r_{*}}$ and $\check{H}_{t}=H_{t}+\dot{\hat{z}} D_{\widehat{r}}$. We argue that $\check{H}_{t} v \in \mathcal{H}$ is equivalent to $H_{t} v \in \mathcal{H}$. Let $\check{H}=H+\dot{\widehat{z}} D_{\widehat{r}}$. From $\check{H}_{t} u \in \mathcal{H}_{t}$ we infer $\check{H}[u]_{H} \in \mathcal{H}$. But for $0<\delta<\epsilon_{1}$ and $\epsilon_{2}>0$ :

$$
\begin{aligned}
\left\|\check{H}[u]_{H}\right\| & \geq\left\|H[u]_{H}\right\|-\left(1-\epsilon_{1}\right)\left\|D_{\widehat{r}}[u]_{H}\right\| \\
& \geq\left(\epsilon_{1}-\delta\right)\left\|D_{\widehat{r}}[u]_{H}\right\|+\epsilon_{2}\left\|a_{0} \not D_{S^{2}}[u]_{H}\right\|-C_{1}\left\|[u]_{H}\right\| \\
& \geq \tilde{\epsilon}\left\|H[u]_{H}\right\|-\widetilde{C}\left\|[u]_{H}\right\|, \quad \tilde{\epsilon}>0 .
\end{aligned}
$$

Here we have used Remark 4.2 and (3.53). The implication $H_{t} u \in \mathscr{H} \Rightarrow \check{H}_{t} u \in \mathcal{H}$ is shown in a similar way. We therefore put $D\left(\check{D}_{t}\right)=D\left(\not_{t}\right)$ and $D\left(\check{H}_{t}\right)=D\left(H_{t}\right)$. Note that $\check{D}_{0}=\not_{0}$ and $\check{H}_{0}=H_{0}$.

LEMMA 4.2. - The operators $\left(\check{D ̆}_{t}, D\left(\check{D}_{t}\right)\right)$ and $\left(\check{H}_{t}, D\left(\check{H}_{t}\right)\right)$ are selfadjoint.

Proof. - The selfadjointness of $\left(\check{H}_{t}, D\left(\check{H}_{t}\right)\right)$ follows from the selfadjointness of $\left(\check{D}_{t}, D\left(\check{D}_{t}\right)\right)$, which we show in the following. We calculate for $u, v \in D\left(\check{D}_{t}\right)$

$$
\begin{aligned}
&\left\langle\check{D}_{t} u, v\right\rangle=\left\langle u, \check{D}_{t} v\right\rangle+\frac{1}{i} \int_{S^{2}}\left\langle\left(-\dot{z}-\Gamma^{1} h^{2}+\left(\partial_{\theta} z\right) a_{0} h^{2} \Gamma^{2}\right) u, v\right\rangle(z(t, \theta), \omega) \mathrm{d} \omega \\
&=--i \int_{S^{2}}\left(-\left(h^{2}+\dot{z}\right) u_{1} \bar{v}_{1}+\left(\partial_{\theta} z\right) a_{0} h^{2} u_{2} \bar{v}_{1}+\left(\partial_{\theta} z\right) a_{0} h^{2} u_{1} \bar{v}_{2}\right)(z(t, \theta), \omega) \mathrm{d} \omega \\
&-i \int_{S^{2}}\left(\left(h^{2}-\dot{z}\right) u_{2} \bar{v}_{2}+\left(h^{2}-\dot{z}\right) u_{3} \bar{v}_{3}-a_{0} h^{2}\left(\partial_{\theta} z\right) u_{4} \bar{v}_{3}\right)(z(t, \theta), \omega) \mathrm{d} \omega \\
&-i \int_{S^{2}}\left(-a_{0} h^{2}\left(\partial_{\theta} z\right) u_{3} \bar{v}_{4}-\left(h^{2}+\dot{z}\right) u_{4} \bar{v}_{4}\right)(z(t, \theta), \omega) \mathrm{d} \omega \\
&=-i \int_{S^{2}} \frac{\mathrm{e}^{i \nu}}{w}\left(-u_{3} \bar{v}_{1}-u_{4} \bar{v}_{2}+u_{3} \bar{v}_{1}+u_{4} \bar{v}_{2}\right)(z(t, \theta), \omega) \mathrm{d} \omega=0 .
\end{aligned}
$$

Therefore $\check{D}_{t}$ is symmetric. We have to show that $D\left(\check{D}_{t}^{*}\right)=D\left(\check{D}_{t}\right)$. We have

$$
v \in D\left(\check{\not D}_{t}^{*}\right) \Longleftrightarrow \forall u \in D\left(\check{D}_{t}\right),\left|\left\langle\check{D}_{t} u, v\right\rangle\right| \leq C\|u\|
$$


Taking $u \in C_{0}^{\infty}\left(\Sigma_{t}^{\text {col }} ; \mathbb{C}^{4}\right)$ in $(4.73)$ we find that $\check{D}_{t} v \in \mathscr{H}_{*}$, thus $v(z(t, \theta), \omega)$ is well defined. For $u \in D\left(\check{\not D}_{t}\right)$ and $v \in D\left(\check{\not}_{t}^{*}\right)$ we compute

$$
\begin{aligned}
\left\langle\check{D}_{t} u, v\right\rangle= & \left\langle u, \check{D}_{t} v\right\rangle+i \int_{S^{2}}\left\langle\left(-\dot{z}-h^{2} \Gamma^{1}+\left(\partial_{\theta} z\right) a_{0} h^{2} \Gamma^{2}\right) u, v\right\rangle(z(t, \theta), \omega) \mathrm{d} \omega \\
= & -i \int_{S^{2}}\left(-\mathrm{e}^{i \nu} u_{3} \bar{v}_{1}-\mathrm{e}^{i \nu} u_{4} \bar{v}_{2}+\left(h^{2}-\dot{z}\right) u_{3} \bar{v}_{3}-a_{0} h^{2}\left(\partial_{\theta} z\right) u_{4} \bar{v}_{3} \mathrm{~d} \omega\right. \\
& \left.\quad+i \int_{S^{2}} a_{0} h^{2}\left(\partial_{\theta} z\right) u_{3} \bar{v}_{4}-\left(h^{2}+\dot{z}\right) u_{4} \bar{v}_{4}\right)(z(t, \theta), \omega) \mathrm{d} \omega+\left\langle u, \check{D}_{t} v\right\rangle \\
= & -i \int_{S^{2}} u_{3} \overline{\left(-\mathrm{e}^{-i \nu} v_{1}+\left(h^{2}-\dot{z}\right) v_{3}-a_{0} h^{2}\left(\partial_{\theta} z\right) v_{4}\right)}(z(t, \theta), \omega) \mathrm{d} \omega \\
& -i \int_{S^{2}} u_{4} \overline{\left(-\mathrm{e}^{-i \nu} v_{2}-\left(\partial_{\theta} z\right) a_{0} h^{2} v_{3}-\left(h^{2}+\dot{z}\right) v_{4}\right)}(z(t, \theta), \omega) \mathrm{d} \omega+\left\langle u, \check{D}_{t} v\right\rangle .
\end{aligned}
$$

It follows from (4.73):

$$
\begin{aligned}
& \mid \int_{S^{2}} u_{3} \overline{\left(-\mathrm{e}^{-i \nu} v_{1}+\left(h^{2}-\dot{z}\right) v_{3}-a_{0} h^{2}\left(\partial_{\theta} z\right) v_{4}\right)}(z(t, \theta), \omega) \mathrm{d} \omega \\
& \quad+\int_{S^{2}} u_{4} \overline{\left(-\mathrm{e}^{-i \nu} v_{2}-\left(\partial_{\theta} z\right) a_{0} h^{2} v_{3}-\left(h^{2}+\dot{z}\right) v_{4}\right)}(z(t, \theta), \omega) \mathrm{d} \omega \mid \leq C\|u\| .
\end{aligned}
$$

Let $\phi \in C_{0}^{\infty}(\mathbb{R}), \phi(0)=1, u_{\epsilon}\left(r_{*}, \omega\right)=\phi\left(\left(r_{*}-z(t, \theta)\right) / \epsilon\right) u\left(r_{*}, \omega\right)$. Clearly

$$
u_{\epsilon}(z(t, \theta), \omega)=u(z(t, \theta), \omega) \text { and } u_{\epsilon} \in D\left(\check{D}_{t}\right) \text {. }
$$

We estimate:

$$
\begin{aligned}
\|u\|^{2}=\int_{S^{2}} \int_{z(t, \theta)}^{\infty}\left|u_{\epsilon}\right|^{2} \mathrm{~d} r_{*} \mathrm{~d} \omega & \lesssim \epsilon\left\|[u]_{H}^{*}\right\|_{L^{\infty}\left(\mathbb{R} ;\left(L^{2}\left(S^{2}\right)\right)^{4}\right)}^{2} \\
& \lesssim \epsilon\left\|[u]_{H}^{*}\right\|_{H^{1}\left(\mathbb{R} ;\left(L^{2}\left(S^{2}\right)\right)^{4}\right)} \lesssim \epsilon\left\|[u]_{H}^{*}\right\|_{\mathcal{H}_{*}^{1}}^{2}
\end{aligned}
$$

Thus if we replace $u$ by $u_{\epsilon}$ in (4.74), then the term on the R.H.S. goes to zero when $\epsilon \rightarrow 0$, whereas the term on the L.H.S. remains unchanged. It follows that, for all $u \in D\left(\check{\not}_{t}\right)$,

$$
\begin{aligned}
\int_{S^{2}} u_{3} \overline{\left(-\mathrm{e}^{-i \nu} v_{1}+\left(h^{2}-\dot{z}\right) v_{3}-a_{0} h^{2}\left(\partial_{\theta} z\right) v_{4}\right)}(z(t, \theta), \omega) \mathrm{d} \omega \\
\quad+\int_{S^{2}} u_{4} \overline{\left(-\mathrm{e}^{-i \nu} v_{2}-\left(\partial_{\theta} z\right) a_{0} h^{2} v_{3}-\left(h^{2}+\dot{z}\right) v_{4}\right)}(z(t, \theta), \omega) \mathrm{d} \omega=0 .
\end{aligned}
$$

Let $\mathcal{R}=\left\{(z(t, \theta), \omega) ; \omega \in S^{2}\right\}$. We claim:

$$
\forall f \in L^{2}\left(\mathscr{R} ; \mathbb{C}^{2}\right), \exists u \in D\left(\check{D}_{t}\right),\left.\quad u_{3,4}\right|_{\mathscr{R}}=f .
$$

From $(4.75),(4.76)$ follows that $v$ satisfies the boundary condition. Therefore it remains to show (4.76). Let

$$
\operatorname{Ker}\left(w \Gamma^{4}\left(-\dot{z}-h^{2} \Gamma^{1}+\left(\partial_{\theta} z\right) a_{0} h^{2} \Gamma^{2}+\frac{i}{w} \Gamma^{4} \mathcal{B}\right)\right)=\operatorname{span}\left\{w_{1}, w_{2}\right\}
$$


with $w_{1}={ }^{t}\left(w_{11}, \ldots, w_{14}\right)$ and $w_{2}={ }^{t}\left(w_{21}, \ldots, w_{24}\right)$. The vectors ${ }^{t}\left(w_{13}, w_{14}\right)$ and ${ }^{t}\left(w_{23}, w_{34}\right)$ are linearly independent. Indeed supposing

$$
\left(\begin{array}{l}
w_{13} \\
w_{14}
\end{array}\right)=\alpha\left(\begin{array}{l}
w_{23} \\
w_{24}
\end{array}\right) \text { we find } M\left(\begin{array}{l}
w_{11} \\
w_{12}
\end{array}\right)=\alpha M\left(\begin{array}{l}
w_{21} \\
w_{22}
\end{array}\right)
$$

with

$$
M=\left(\begin{array}{ll}
-\left(\dot{z}+h^{2}\right) & a_{0} h^{2}\left(\partial_{\theta} z\right) \\
a_{0} h^{2}\left(\partial_{\theta} z\right) & h^{2}-\dot{z}
\end{array}\right) .
$$

The matrix $M$ being invertible we find

$$
\left(\begin{array}{l}
w_{11} \\
w_{12}
\end{array}\right)=\alpha\left(\begin{array}{l}
w_{21} \\
w_{22}
\end{array}\right)
$$

which is a contradiction. If $f \in L^{2}\left(\mathscr{R} ; \mathbb{C}^{2}\right)$, define $g \in L^{2}\left(\mathscr{R} ; \mathbb{C}^{4}\right)$ in the following way:

$$
\exists \alpha, \beta \quad \alpha\left(\begin{array}{l}
w_{13} \\
w_{14}
\end{array}\right)+\beta\left(\begin{array}{l}
w_{23} \\
w_{24}
\end{array}\right)=f .
$$

We put $g:=\alpha w_{1}+\beta w_{2}$. By the surjectivity of the trace operator there exists $u \in \mathcal{H}_{* t}^{1}$ such that $\left.u\right|_{\mathscr{R}}=g$. By construction of $g, u \in D\left(\widetilde{D}_{t}\right)$.

The problem (4.71) is solved by the following proposition.

Proposition 4.5. - Let $\Psi_{s} \in D\left(H_{s}\right)$. Then there exists a unique solution

$$
[\Psi(.)]_{H}=\left[U(., s) \Psi_{s}\right]_{H} \in C^{1}\left(\mathbb{R}_{t} ; \mathcal{H}\right) \cap C\left(\mathbb{R}_{t} ; \mathcal{H}^{1}\right)
$$

of (4.71) such that for all $t \in \mathbb{R} \Psi(t) \in D\left(H_{t}\right)$. Furthermore we have $\|\Psi(t)\|=\left\|\Psi_{s}\right\|$ and $U(t, s)$ possesses an extension to an isometric and strongly continuous propagator from $\mathcal{H}_{s}$ to $\mathcal{H}_{t}$ such that for all $\Psi_{s} \in D\left(H_{s}\right)$ we have

$$
\frac{\mathrm{d}}{\mathrm{d} t} U(t, s) \Psi_{s}=i H_{t} U(t, s) \Psi_{s} .
$$

The proposition follows from Proposition 8.2, which is proven in the appendix. 



\section{CHAPTER 5}

\section{DIRAC QUANTUM FIELDS}

We adopt the approach of Dirac quantum fields in the spirit of [16] and [17]. This approach is explained in Section 5.2. In Section 5.1 we recall the second quantization of Dirac fields (see e.g. [11] for a detailed discussion of the second quantization procedure and [46] for the special case of the Dirac equation). In Section 5.3 we present the theorem about the Hawking effect.

\subsection{Second Quantization of Dirac Fields}

We first explain the construction in the case of one kind of noninteracting fermions. The one fermion space is a complex Hilbert space $\mathfrak{H}$ with scalar product $\langle.,$.$\rangle that$ we suppose linear with respect to the first argument. The space of $n$ fermions is the antisymmetric $n$-tensor product of $\mathfrak{H}$ :

$$
\mathcal{F}^{(0)}(\mathfrak{H}):=\mathbb{C}, 1 \leq n \Longrightarrow \mathcal{F}^{(n)}(\mathfrak{H}):=\bigwedge_{\nu=1}^{n} \mathfrak{H} .
$$

The Fermi-Fock space is defined as

$$
\mathcal{F}(\mathfrak{H}):=\bigoplus_{n=0}^{\infty} \mathcal{F}^{(n)}(\mathfrak{H}) .
$$

For $f \in \mathfrak{H}$ we construct the fermion annihilation operator $a_{\mathfrak{H}}(f)$, and the fermion creation operator $a_{\mathfrak{H}}^{*}(f)$ by putting

$$
\begin{gathered}
a_{\mathfrak{H}}(f): \mathcal{F}^{(0)}(\mathfrak{H}) \longrightarrow\{0\}, 1 \leq n, \quad a_{\mathfrak{H}}(f): \mathcal{F}^{(n)}(\mathfrak{H}) \longrightarrow \mathcal{F}^{(n-1)}(\mathfrak{H}), \\
a_{\mathfrak{H}}(f)\left(f_{1} \wedge \cdots \wedge f_{n}\right)=\frac{\sqrt{n}}{n !} \sum_{\sigma} \epsilon(\sigma)\left\langle f_{\sigma(1)}, f\right\rangle f_{\sigma(2)} \wedge \cdots \wedge f_{\sigma(n)}, \\
0 \leq n, \quad a_{\mathfrak{H}}^{*}(f): \mathcal{F}^{(n)}(\mathfrak{H}) \longrightarrow \mathcal{F}^{(n+1)}(\mathfrak{H}), \\
a_{\mathfrak{H}}^{*}(f)\left(f_{1} \wedge \cdots \wedge f_{n}\right)=\frac{\sqrt{n+1}}{n !} \sum_{\sigma} \epsilon(\sigma) f \wedge f_{\sigma(1)} \wedge \cdots \wedge f_{\sigma(n)},
\end{gathered}
$$


where the sum runs over all permutations $\sigma$ of $\{1, \ldots, n\}$ and $\epsilon(\sigma)$ is one if $\sigma$ is even and -1 if $\sigma$ is odd. In contrast to the boson case, $a_{\mathfrak{H}}(f)$ and $a_{\mathfrak{H}}^{*}(f)$ have bounded extensions on $\mathcal{F}(\mathfrak{H})$, which we still denote $a_{\mathfrak{H}}(f), a_{\mathfrak{H}}^{*}(f)$. They satisfy:

$$
\begin{aligned}
\left\|a_{\mathfrak{H}}(f)\right\| & =\left\|a_{\mathfrak{H}}^{*}(f)\right\|=\|f\|, \\
a_{\mathfrak{H}}^{*}(f) & =\left(a_{\mathfrak{H}}(f)\right)^{*} .
\end{aligned}
$$

Another important feature of the creation and annihilation operators is that they satisfy the canonical anti-commutation relations (CAR's):

$$
\begin{aligned}
& a_{\mathfrak{H}}(f) a_{\mathfrak{H}}(g)+a_{\mathfrak{H}}(g) a_{\mathfrak{H}}(f)=0, \\
& a_{\mathfrak{H}}^{*}(f) a_{\mathfrak{H}}^{*}(g)+a_{\mathfrak{H}}^{*}(g) a_{\mathfrak{H}}^{*}(f)=0, \\
& a_{\mathfrak{H}}^{*}(f) a_{\mathfrak{H}}(g)+a_{\mathfrak{H}}(g) a_{\mathfrak{H}}^{*}(f)=\langle f, g\rangle \mathbf{1} .
\end{aligned}
$$

The CAR algebra on $\mathfrak{H}$ is the $C^{*}$-algebra $\mathcal{U}(\mathfrak{H})$ generated by the identity and the $a_{\mathfrak{H}}(f), f \in \mathfrak{H}$.

Let us now consider a situation where the classical fields obey the Schrödinger type equation $(H$ selfadjoint on $\mathfrak{H})$ :

$$
\partial_{t} \Psi=i H \Psi
$$

A gauge invariant quasi-free state $\omega$ on $\mathcal{U}(\mathfrak{H})$ satisfies the $(\beta, \mu)$-KMS condition, $0<\beta$, if it is characterized by the two point function

$$
\omega\left(a_{\mathfrak{H}}^{*}(f) a_{\mathfrak{H}}(f)\right)=\left\langle z \mathrm{e}^{\beta H}\left(1+z \mathrm{e}^{\beta H}\right)^{-1} f, g\right\rangle,
$$

where $z$ is the activity given by $z=\mathrm{e}^{\beta \mu}$. This state is a model for the ideal Fermi gas with temperature $0<T=\beta^{-1}$ and chemical potential $\mu$.

In the case of charged spinor fields we have to consider both kinds of fermions, the particles and the antiparticles. The space of the classical charged spinor fields is given by a complex Hilbert space $\mathfrak{H}$ together with an anti-unitary operator $\Upsilon$ on $\mathfrak{H}$ (the charge conjugation). We assume $\mathfrak{H}$ is split into two orthogonal subspaces:

$$
\mathfrak{H}=\mathfrak{H}_{+} \oplus \mathfrak{H}_{-} \cdot
$$

We define the one particle space

$$
\mathfrak{h}_{+}=\mathfrak{H}_{+}
$$

and the one antiparticle space

$$
\mathfrak{h}_{-}=\Upsilon \mathfrak{H}_{-} .
$$

The space of $n$ particles and $m$ antiparticles is given by the tensor product of the previous spaces:

$$
\mathcal{F}^{(n, m)}:=\mathcal{F}^{(n)}\left(\mathfrak{h}_{+}\right) \otimes \mathcal{F}^{(m)}\left(\mathfrak{h}_{-}\right)
$$


The Dirac-Fermi-Fock space is given by

$$
\mathcal{F}(\mathfrak{H}):=\bigoplus_{n, m=0}^{\infty} \mathcal{F}^{(n, m)} .
$$

We will denote the elements $\Psi$ of $\mathcal{F}(\mathfrak{H})$ by sequences

$$
\Psi=\left(\Psi^{(n, m)}\right)_{n, m \in \mathbb{N}}, \quad \Psi^{(n, m)} \in \mathcal{F}^{(n, m)},
$$

the vacuum vector is the vector $\Omega_{\mathrm{vac}}$ defined by

$$
\Omega_{\text {vac }}^{(0,0)}=1, \quad(n, m) \neq(0,0) \Longrightarrow \Omega_{\text {vac }}^{(n, m)}=0 .
$$

For $\phi_{ \pm} \in \mathfrak{h}_{ \pm}$we define the particle annihilation operator, a $\left(\phi_{+}\right)$, the particle creation operator, $a^{*}\left(\phi_{+}\right)$, the antiparticle annihilation operator, $b\left(\phi_{-}\right)$, and the antiparticle creation operator, $b^{*}\left(\phi_{-}\right)$, by putting for $\Psi_{+}^{(n)} \otimes \Psi_{-}^{(m)} \in \mathcal{F}^{(n, m)}$ :

$$
\begin{aligned}
a\left(\phi_{+}\right)\left(\Psi_{+}^{(n)} \otimes \Psi_{-}^{(m)}\right) & =\left(a_{\mathfrak{h}_{+}}\left(\phi_{+}\right)\left(\Psi_{+}^{(n)}\right)\right) \otimes \Psi_{-}^{(m)} \in \mathcal{F}^{(n-1, m)}, \\
a^{*}\left(\phi_{+}\right)\left(\Psi_{+}^{(n)} \otimes \Psi_{-}^{(m)}\right) & =\left(a_{\mathfrak{h}_{+}}^{*}\left(\phi_{+}\right)\left(\Psi_{+}^{(n)}\right)\right) \otimes \Psi_{-}^{(m)} \in \mathcal{F}^{(n+1, m)}, \\
b\left(\phi_{-}\right)\left(\Psi_{+}^{(n)} \otimes \Psi_{-}^{(m)}\right) & =\Psi_{+}^{(n)} \otimes\left(b_{\mathfrak{h}_{-}}\left(\phi_{-}\right)\left(\Psi_{-}^{(m)}\right)\right) \in \mathcal{F}^{(n, m-1)}, \\
b^{*}\left(\phi_{-}\right)\left(\Psi_{+}^{(n)} \otimes \Psi_{-}^{(m)}\right) & =\Psi_{+}^{(n)} \otimes\left(b_{\mathfrak{h}_{-}}^{*}\left(\phi_{-}\right)\left(\Psi_{-}^{(m)}\right)\right) \in \mathcal{F}^{(n, m+1)} .
\end{aligned}
$$

All these operators have bounded extensions on $\mathcal{F}(\mathfrak{H})$ and satisfy the CAR's. The main object of the theory is the quantized Dirac field operator $\Psi$ :

$$
f \in \mathfrak{H} \longmapsto \Psi(f):=a\left(\Pi_{+} f\right)+b^{*}\left(\Upsilon \Pi_{-} f\right) \in \mathscr{L}(\mathcal{F}(\mathfrak{H})),
$$

where we have denoted by $\Pi_{ \pm}$the orthogonal projector from $\mathfrak{H}$ to $\mathfrak{H}_{ \pm}$. The mapping $f \mapsto \Psi(f)$ is antilinear and bounded:

$$
\|\Psi(f)\|=\|f\| .
$$

Its adjoint denoted by $\Psi^{*}(f)$ is given by

$$
\Psi^{*}(f)=a^{*}\left(\Pi_{+} f\right)+b\left(\Upsilon \Pi_{-} f\right)
$$

and the CAR's are satisfied:

$$
\begin{aligned}
\Psi(f) \Psi(g)+\Psi(g) \Psi(f) & =0, \\
\Psi^{*}(f) \Psi^{*}(g)+\Psi^{*}(g) \Psi^{*}(f) & =0, \\
\Psi^{*}(f) \Psi(g)+\Psi(g) \Psi^{*}(f) & =\langle f, g\rangle \mathbf{1} .
\end{aligned}
$$

The Field Algebra is the $C^{*}$-algebra generated by $\mathbf{1}$ and the $\Psi(f), f \in \mathfrak{H}$. If we take $f$ only in $\mathfrak{H}_{+(-)}$we get a subalgebra isometric to $\mathcal{U}\left(\mathfrak{h}_{+(-)}\right)$. The vacuum state $\omega_{\text {vac }}$ is defined by

$$
A \in \mathcal{U}(\mathfrak{H}), \quad \omega_{\mathrm{vac}}(A):=\left\langle A \Omega_{\mathrm{vac}}, \Omega_{\mathrm{vac}}\right\rangle,
$$

or by the two point function

$$
\omega_{\mathrm{vac}}\left(\Psi^{*}(f) \Psi(g)\right)=\left\langle\Pi_{-} f, \Pi_{-} g\right\rangle .
$$


Now assume that the classical fields satisfy a Dirac type equation:

$$
\partial_{t} \Psi=i H \Psi
$$

where $H$ is selfadjoint on $\mathfrak{H}$ and leaves $\mathfrak{H}_{+}, \mathfrak{H}_{-}$invariant. Then

$$
H^{+}:=\left.H\right|_{\mathfrak{h}_{+}}, \quad H^{-}:=-\left.\Upsilon H\right|_{\mathfrak{H}_{-}} \Upsilon^{-1},
$$

are respectively selfadjoint on $\mathfrak{h}_{+}$and $\mathfrak{h}_{-}$, and the classical fields of one particle, $\phi_{+}$, and of one antiparticle, $\phi_{-}$, are solutions to a Schrödinger type equation on $\mathfrak{h}_{+(-)}$:

$$
\partial_{t} \phi_{+(-)}=i H^{+(-)} \phi_{+(-)} .
$$

A usual splitting of $\mathfrak{H}$ is the choice

$$
\mathfrak{H}_{+}=\mathbf{1}_{(-\infty, 0)}(H), \quad \mathfrak{H}_{-}=\mathbf{1}_{[0, \infty)}(H) .
$$

We say that a state $\omega_{\beta, \mu}$ on $\mathcal{U}(\mathfrak{H})$ satisfies the $(\beta, \mu)$-KMS condition, $0<\beta$ if it is characterized by the two-point function

$$
\omega_{\beta, \mu}\left(\Psi^{*}(f) \Psi(g)\right)=\left\langle z \mathrm{e}^{\beta H}\left(1+z \mathrm{e}^{\beta H}\right)^{-1} f, g\right\rangle, \quad z=\mathrm{e}^{\beta \mu} .
$$

We want to apply this procedure to several states at time $t=0$ and in the future. We first describe the quantization at time $t=0$. Let $\mathfrak{H}=\mathscr{H}_{0}, H=H_{0}$. We will emphasize the importance of the charge of the field by denoting the hamiltonian $H_{0}=H_{0}(q)$. A charge conjugation for $H_{0}(q)$ is given by

$$
\Upsilon \phi=U_{\Upsilon} \bar{\phi} \quad \text { with } \quad U_{\Upsilon}=\gamma^{3}=\left(\begin{array}{rrrr}
0 & 0 & 0 & 1 \\
0 & 0 & -1 & 0 \\
0 & 1 & 0 & 0 \\
-1 & 0 & 0 & 0
\end{array}\right) .
$$

We note that $H^{-}=H_{0}(-q)$ and that $\psi=\Upsilon \phi$ satisfies the boundary condition (4.68) if $\phi$ satisfies it.

As we do not know whether $H_{0}$ has the eigenvalue 0 or not, there is a slight ambiguity in the definition of particles and antiparticles. We will put

$$
\left(\Pi_{+}, \Pi_{-}\right)=\left(\mathbf{1}_{(-\infty, 0)}\left(H_{0}\right), \mathbf{1}_{[0, \infty)}\left(H_{0}\right)\right),
$$

but the choice $\left(\mathbf{1}_{(-\infty, 0]}\left(H_{0}\right), \mathbf{1}_{(0, \infty)}\left(H_{0}\right)\right)$ would also have been possible. We denote $\Psi_{0}$ the quantum field at time $t=0$ constructed in the previous way. We define the Boulware quantum state $\omega_{0}$ on the field algebra $\mathcal{U}\left(\mathcal{H}_{0}\right)$ as the vacuum state

$$
\Phi_{0}^{j} \in \mathcal{H}_{0} \quad \omega_{0}\left(\Psi_{0}^{*}\left(\Phi_{0}^{1}\right) \Psi_{0}\left(\Phi_{0}^{2}\right)\right)=\left\langle\Pi_{-} \Phi_{0}^{1}, \Pi_{-} \Phi_{0}^{2}\right\rangle .
$$

At time $t=\infty$ we will take

$$
\mathfrak{H}=\mathscr{H},
$$

$H$ the Dirac hamiltonian in the Kerr-Newman space-time and the same charge conjugation. We put

$$
\left(\Pi_{+}, \Pi_{-}\right)=\left(\mathbf{1}_{(-\infty, 0)}(H), \mathbf{1}_{[0, \infty)}(H)\right)
$$


The fields obtained in this way are denoted $\Psi(f)$. From this we obtain the definitions of vacuum and KMS states with respect to $H$.

\subsection{Quantization in a globally hyperbolic space-time}

Following J. Dimock [17] we construct the local algebra of observables in the spacetime outside the collapsing star. This construction does not depend on the choice of the representation of the CAR's, or on the spin structure of the Dirac field, or on the choice of the hypersurface. In particular we can consider the Fermi-Dirac-Fock representation and the following foliation of our space-time (see Section 3.2):

$$
\mathcal{M}_{\mathrm{col}}=\bigcup_{t \in \mathbb{R}} \Sigma_{t}^{\mathrm{col}}, \quad \Sigma_{t}^{\mathrm{col}}=\{(t, \widehat{r}, \theta, \varphi) ; \widehat{r} \geq \widehat{z}(t, \theta)\} .
$$

We construct the Dirac field $\Psi_{0}$ and the $C^{*}$-algebra $\mathcal{U}\left(\mathcal{H}_{0}\right)$ as explained in Section 5.1. We define the operator

$$
S_{\mathrm{col}}: \Phi \in\left(C_{0}^{\infty}\left(\mathcal{M}_{\mathrm{col}}\right)\right)^{4} \longmapsto S_{\mathrm{col}} \Phi:=\int_{\mathbb{R}} U(0, t) \Phi(t) \mathrm{d} t \in \mathcal{H}_{0},
$$

where $U(0, t)$ is the propagator defined in Proposition 4.5. The quantum spin field is defined by

$$
\Psi_{\mathrm{col}}: \Phi \in\left(C_{0}^{\infty}\left(\mathcal{M}_{\mathrm{col}}\right)\right)^{4} \longmapsto \Psi_{\mathrm{col}}(\Phi):=\Psi_{0}\left(S_{\mathrm{col}} \Phi\right) \in \mathscr{L}\left(\mathcal{H}_{0}\right)
$$

and for an arbitrary set $\theta \subset \mathcal{M}_{\text {col }}$, we introduce $\mathcal{U}_{\text {col }}(\theta)$, the $C^{*}$-algebra generated by $\psi_{\text {col }}^{*}\left(\Phi_{1}\right) \Psi_{\text {col }}\left(\Phi_{2}\right)$, supp $\Phi_{j} \subset \vartheta, j=1,2$. Eventually, we have

$$
u_{\mathrm{col}}\left(\mathcal{M}_{\mathrm{col}}\right)=\overline{\bigcup_{\theta \subset \mathcal{M}_{\mathrm{col}}} u_{\mathrm{col}}(\theta)} .
$$

Then we define the fundamental state on $\mathcal{U}_{\text {col }}\left(\mathcal{M}_{\text {col }}\right)$ as follows:

$\omega_{\mathrm{col}}\left(\Psi_{\mathrm{col}}^{*}\left(\Phi_{1}\right) \Psi_{\mathrm{col}}\left(\Phi_{2}\right)\right):=\omega_{\mathrm{vac}}\left(\Psi_{0}^{*}\left(S_{\mathrm{col}} \Phi_{1}\right) \Psi_{0}\left(S_{\mathrm{col}} \Phi_{2}\right)\right)=\left\langle\mathbf{1}_{[0, \infty)}\left(H_{0}\right) S_{\mathrm{col}} \Phi_{1}, S_{\mathrm{col}} \Phi_{2}\right\rangle$.

Let us now consider the future black hole. We consider the space-time $\mathcal{M}_{\mathrm{BH}}$ with the Dirac hamiltonian $H$ for a field with one particle. Let $\Psi(\Phi)$ be the Dirac field as constructed in Section 5.1 and

$$
S: \Phi \in\left(C_{0}^{\infty}\left(\mathcal{M}_{\mathrm{BH}}\right)\right)^{4} \longmapsto S \Phi:=\int_{\mathbb{R}} \mathrm{e}^{-i t H} \Phi(t) \mathrm{d} t .
$$

We also introduce:

$$
\Psi_{\mathrm{BH}}: \Phi \in\left(C_{0}^{\infty}\left(\mathcal{M}_{\mathrm{BH}}\right)\right)^{4} \mapsto \Psi_{\mathrm{BH}}(\Phi):=\Psi(S \Phi)
$$

and the $C^{*}$-algebra $\mathcal{U}_{\mathrm{BH}}(\theta)$ generated by $\Psi_{\mathrm{BH}}\left(\Phi_{1}\right) \Psi_{\mathrm{BH}}^{*}\left(\Phi_{2}\right), \Phi_{1}, \Phi_{2} \in\left(C_{0}^{\infty}(\theta)\right)^{4}$, $\theta \subset \mathcal{M}_{\mathrm{BH}}$. As before we put

$$
u_{\mathrm{BH}}\left(\mathcal{M}_{\mathrm{BH}}\right)=\overline{\bigcup_{\theta \subset \mathcal{M}_{\mathrm{BH}}} u_{\mathrm{BH}}(\theta)} .
$$


We also define the thermal Hawking state

$$
\begin{aligned}
\omega_{\text {Haw }}^{\eta, \sigma}\left(\Psi_{\mathrm{BH}}^{*}\left(\Phi_{1}\right) \Psi_{\mathrm{BH}}\left(\Phi_{2}\right)\right) & =\left\langle\mu \mathrm{e}^{\sigma H}\left(1+\mu \mathrm{e}^{\sigma H}\right)^{-1} S \Phi_{1}, S \Phi_{2}\right\rangle_{\mathcal{H}} \\
& =: \omega_{K M S}^{\eta, \sigma}\left(\Psi^{*}\left(S \Phi_{1}\right) \Psi\left(S \Phi_{2}\right)\right)
\end{aligned}
$$

with

$$
T_{\text {Haw }}=\sigma^{-1}, \quad \mu=\mathrm{e}^{\sigma \eta}, \quad \sigma>0,
$$

where $T_{\text {Haw }}$ is the Hawking temperature and $\mu$ is the chemical potential. We will also need a vacuum state which is given by

$$
\omega_{\mathrm{vac}}\left(\Psi_{\mathrm{BH}}^{*}\left(\Phi_{1}\right) \Psi_{\mathrm{BH}}\left(\phi_{2}\right)\right)=\left\langle\mathbf{1}_{[0, \infty)}(H) S \phi_{1}, S \phi_{2}\right\rangle .
$$

\subsection{The Hawking effect}

In this section we formulate the main result of this paper.

Let $\Phi \in\left(C_{0}^{\infty}\left(\mathcal{M}_{\mathrm{col}}\right)\right)^{4}$. We put

$$
\Phi^{T}(t, \widehat{r}, \omega)=\Phi(t-T, \widehat{r}, \omega) .
$$

TheOREM 5.1 (Hawking effect). - Let

$$
\Phi_{j} \in\left(C_{0}^{\infty}\left(\mathcal{M}_{\mathrm{col}}\right)\right)^{4}, \quad j=1,2 .
$$

Then we have

$$
\begin{aligned}
& \lim _{T \rightarrow \infty} \omega_{\text {col }}\left(\Psi_{\text {col }}^{*}\left(\Phi_{1}^{T}\right) \Psi_{\mathrm{col}}\left(\Phi_{2}^{T}\right)\right) \\
&=\omega_{\text {Haw }}^{\eta, \sigma}\left(\Psi_{\mathrm{BH}}^{*}\left(\mathbf{1}_{\mathbb{R}^{+}}\left(P^{-}\right) \Phi_{1}\right) \Psi_{\mathrm{BH}}\left(\mathbf{1}_{\mathbb{R}^{+}}\left(P^{-}\right) \Phi_{2}\right)\right) \\
& \quad \quad+\omega_{\mathrm{vac}}\left(\Psi_{\mathrm{BH}}^{*}\left(\mathbf{1}_{\mathbb{R}^{-}}\left(P^{-}\right) \Phi_{1}\right) \Psi_{\mathrm{BH}}\left(\mathbf{1}_{\mathbb{R}^{-}}\left(P^{-}\right) \Phi_{2}\right)\right), \\
& T_{\text {Haw }}=\frac{1}{\sigma}=\frac{\kappa_{+}}{2 \pi}, \quad \mu=\mathrm{e}^{\sigma \eta}, \quad \eta=\frac{q Q r_{+}}{r_{+}^{2}+a^{2}}+\frac{a D_{\varphi}}{r_{+}^{2}+a^{2}} .
\end{aligned}
$$

In the above theorem $P^{ \pm}$is the asymptotic velocity introduced in Chapter 4 . The projections $\mathbf{1}_{\mathbb{R}^{ \pm}}\left(P^{ \pm}\right)$separate outgoing and incoming solutions.

REMARK 5.1. - The result is independent of the choices of coordinate system and tetrad, i.e. both sides of (5.27) are independent of these choices. Indeed a change of coordinate system or a change of tetrad is equivalent to a conjugation of the operators by a unitary transformation. We also note that the result is independent of the chiral angle $\nu$ in the boundary condition. Let

$$
S_{\leftarrow} \phi=\int_{\mathbb{R}} \mathrm{e}^{-i t H \leftarrow \phi(t) \mathrm{d} t .}
$$

It is easy to check

$$
\begin{aligned}
\omega_{\mathrm{Haw}}^{\eta, \sigma}\left(\Psi_{\mathrm{BH}}^{*}\left(\mathbf{1}_{\mathbb{R}^{+}}\left(P^{-}\right) \phi_{1}\right) \Psi_{\mathrm{BH}}\left(\mathbf{1}_{\mathbb{R}^{+}}\left(P^{-}\right) \phi_{2}\right)\right) \\
=\left\langle\mu \mathrm{e}^{\sigma H_{\leftarrow}}\left(1+\mathrm{e}^{\sigma H_{\leftarrow}}\right)^{-1} S_{\leftarrow} \Omega_{\leftarrow}^{-} \phi_{1}, S_{\leftarrow} \Omega_{\leftarrow}^{-} \phi_{2}\right\rangle .
\end{aligned}
$$


We define $S_{\rightarrow}$ in an analogous way to $S_{\leftarrow}$ using the Dollard modified dynamics:

$$
S_{\rightarrow} \phi=\int_{\mathbb{R}} U_{D}(-t) \phi(t) \mathrm{d} t .
$$

We find

$$
\omega_{\text {vac }}\left(\Psi_{\mathrm{BH}}^{*}\left(\mathbf{1}_{\mathbb{R}^{-}}\left(P^{-}\right) \phi_{1}\right) \Psi_{\mathrm{BH}}\left(\mathbf{1}_{\mathbb{R}^{-}}\left(P^{-}\right) \phi_{2}\right)=\left\langle\mathbf{1}_{[0, \infty)}\left(H_{\rightarrow}\right) S_{\rightarrow} \Omega_{\rightarrow}^{-} \phi_{1}, S_{\rightarrow} \Omega_{\rightarrow}^{-} \phi_{2}\right\rangle .\right.
$$

In particular our result coincides with the result of Melnyk [37] in the case of a Reissner-Nordström black hole. 



\section{CHAPTER 6}

\section{ADDITIONAL SCATTERING RESULTS}

In this chapter we state some scattering results that we shall need in what follows.

\subsection{Spin weighted spherical harmonics}

We will now introduce spin weighted spherical harmonics $Y_{\mathrm{sn}}^{l}$ (for a complete definition, see e.g. [39]). For each spinorial weight $s, 2 s \in \mathbb{Z}$, the family

$$
\left\{Y_{\mathrm{sn}}^{\ell}(\varphi, \theta)=\mathrm{e}^{i n \varphi} u_{\mathrm{sn}}^{\ell}(\theta) ; \ell-|s| \in \mathbb{N}, \ell-|n| \in \mathbb{N}\right\}
$$

forms a Hilbert basis of $L^{2}\left(S_{\omega}^{2}, \mathrm{~d} \omega\right)$ and we have the relations

$$
\begin{aligned}
& \frac{\mathrm{d} u_{\mathrm{sn}}^{\ell}}{\mathrm{d} \theta}-\frac{n-s \cos \theta}{\sin \theta} u_{\mathrm{sn}}^{\ell}=-i[(\ell+s)(\ell-s+1)]^{\frac{1}{2}} u_{s-1, n}^{\ell}, \\
& \frac{\mathrm{d} u_{\mathrm{sn}}^{\ell}}{\mathrm{d} \theta}+\frac{n-s \cos \theta}{\sin \theta} u_{\mathrm{sn}}^{\ell}=-i[(\ell+s+1)(\ell-s)]^{\frac{1}{2}} u_{s+1, n}^{\ell} .
\end{aligned}
$$

We define $\otimes_{4}$ as the following operation between two vectors of $\mathbb{C}^{4}$

$$
\forall v=\left(v_{1}, v_{2}, v_{3}, v_{4}\right), u=\left(u_{1}, u_{2}, u_{3}, u_{4}\right), \quad v \otimes_{4} u=\left(u_{1} v_{1}, u_{2} v_{2}, u_{3} v_{3}, u_{4} v_{4}\right) .
$$

Since the families

$$
\left\{Y_{\frac{1}{2}, n}^{\ell} ;(n, \ell) \in \mathcal{J}\right\}, \quad\left\{Y_{-\frac{1}{2}, n}^{\ell} ;(n, \ell) \in \mathcal{J}\right\}, \quad I=\left\{(n, \ell) ; \ell-\frac{1}{2} \in \mathbb{N}, \ell-|n| \in \mathbb{N}\right\}
$$

form a Hilbert basis of $L^{2}\left(S_{\omega}^{2}, d \omega\right)$, we express $\mathcal{H}_{*}$ as a direct sum

$$
\begin{gathered}
\mathcal{H}_{*}=\bigoplus_{(n, \ell) \in \mathcal{J}} \mathcal{H}_{*}^{n \ell}, \quad \mathcal{H}_{*}^{n \ell}=L^{2}\left(\left(\mathbb{R} ; \mathrm{d} r_{*}\right) ; \mathbb{C}^{4}\right) \otimes_{4} Y_{n \ell}, \\
Y_{n \ell}=\left(Y_{-\frac{1}{2}, n}^{\ell}, Y_{\frac{1}{2}, n}^{\ell}, Y_{-\frac{1}{2}, n}^{\ell}, Y_{\frac{1}{2}, n}^{\ell}\right) .
\end{gathered}
$$

We shall henceforth identify $\mathcal{H}_{*}^{n \ell}$ and $L^{2}\left((\mathbb{R} ; \mathrm{d} r) ; \mathbb{C}^{4}\right)$ as well as $\psi_{n \ell} \otimes_{4} Y_{n \ell}$ and $\psi_{n \ell}$. We see that

$$
\not_{\mathfrak{s}}=\bigoplus_{(n, \ell) \in \mathcal{J}} \not_{\mathfrak{s}}^{n \ell} \quad \text { with } \quad \not_{\mathfrak{s}}^{n \ell}:=\Gamma^{1} D_{r_{*}}+a_{0}\left(r_{*}\right) \Gamma^{2}\left(\ell+\frac{1}{2}\right)+b_{0}\left(r_{*}\right) \Gamma^{\nu}+c^{n} .
$$


In a similar way we find the decompositions

$$
\mathbb{D}_{H}=\bigoplus_{(n, \ell) \in \mathcal{J}} \mathbb{D}_{H}^{n \ell}, \quad \mathbb{D}_{\rightarrow}=\bigoplus_{(n, \ell) \in \mathcal{I}} \mathbb{D}_{\rightarrow}^{n \ell} .
$$

Note that the operator $\mathbb{D}_{\mathfrak{s}}^{n \ell}$ is selfadjoint on $\mathcal{H}^{n \ell}$ with domain $\left(H^{1}(\mathbb{R})\right)^{4} \otimes_{4} Y_{n \ell}$. This leads to a useful characterization of the domain $D(\not D)$ (see[15]):

$$
\mathcal{H}_{*}^{1}=D(\not D)=D\left(\not_{\mathfrak{s}}\right)=\left\{u=\sum_{n \ell} u_{n \ell} ; \forall n, \ell, u_{n \ell} \in H^{1}(\mathbb{R}), \sum_{n, l}\left\|\not_{\mathfrak{s}}^{n \ell} u_{n \ell}\right\|^{2}<\infty\right\} .
$$

REMARK 6.1. - Note that $n \in \mathbb{Z}+\frac{1}{2}$. Indeed we are working with quantities of spin weight $\frac{1}{2}$. Such quantities are multiplied by $\mathrm{e}^{\frac{1}{2} i \varphi}$ under rotation of angle $\varphi$. In particular $Y_{\frac{1}{2}, n}^{\ell}$ are not smooth on $S_{\omega}^{2}$, but they are smooth on $[0,2 \pi]_{\varphi} \times[0, \pi]_{\theta}$. Using the axial symmetry of our equations we will often fix the angular momentum $D_{\varphi}=n$. When we do so we will always suppose $n \in \mathbb{Z}+\frac{1}{2}$.

\subsection{Velocity estimates}

We start with the maximal velocity estimate:

Lemma 6.1. - Let $\left.\left.J \in C_{b}^{\infty}(\mathbb{R}), \operatorname{supp} J \subset\right]-\infty,-1-\epsilon\right] \cup[1+\epsilon, \infty[$ for some $\epsilon>0$. Then we have

$$
\int_{1}^{\infty}\left\|J\left(\frac{\widehat{r}}{t}\right) \mathrm{e}^{i t H} \phi\right\|^{2} \frac{\mathrm{d} t}{t} \lesssim\|\phi\|^{2} \quad \text { and } \quad s-\lim _{t \rightarrow \pm \infty} J\left(\frac{\widehat{r}}{t}\right) \mathrm{e}^{i t H}=0 .
$$

The lemma can be easily deduced from the equivalent statement for the dynamics $\not D$ in [15, Proposition 4.4]. The minimal velocity estimate is given by the following lemma:

Lemma 6.2. - Let $\chi \in C_{0}^{\infty}(\mathbb{R})$ such that $\operatorname{supp} \chi \subset \mathbb{R} \backslash\{-m, m\}$. Then there exists a strictly positive constant $\epsilon_{\chi}$ such that we have

$$
\int_{1}^{\infty}\left\|\mathbf{1}_{\left[0, \epsilon_{\chi}\right]}\left(\frac{|\widehat{r}|}{t}\right) \mathrm{e}^{i t H} \chi(H) \Phi\right\|^{2} \frac{\mathrm{d} t}{t} \leq C_{\chi}\|\Phi\|^{2} .
$$

Furthermore

$$
s-\lim _{t \rightarrow \infty} \mathbf{1}_{\left[0, \epsilon_{\chi}\right]}\left(\frac{|\widehat{r}|}{t}\right) \mathrm{e}^{i t H} \chi(H)=0 .
$$

The lemma follows from [15, Proposition 4.3]. The change of variables and tetrads is treated in the usual way. It turns out that we need a stronger minimal velocity estimate near the horizon: 
Lemma 6.3. - Let $m \in C\left(\mathbb{R}^{+}\right), 0<\epsilon<1, \lim _{t \rightarrow \infty} m(t)=\infty, 0 \leq m(|t|) \leq \epsilon|t|$ for all $|t| \geq 1$. Then

$$
s-\lim _{t \rightarrow \pm \infty} \mathbf{1}_{[0,1]}\left(\frac{|\widehat{r}|}{|t|-m(|t|)}\right) \mathrm{e}^{i t H} \mathbf{1}_{\mathbb{R} \mp}\left(P^{ \pm}\right)=0 .
$$

An analogous result holds if we replace $\mathrm{e}^{i t H} \mathbf{1}_{\mathbb{R}^{\mp}}\left(P^{ \pm}\right)$by $\mathrm{e}^{i t H_{\leftarrow}}$.

Proof. - By the asymptotic completeness result and a density argument it is sufficient to show:

$$
\forall f \in\left(C_{0}^{\infty}\left(\mathbb{R} \times S^{2}\right)\right)^{4}, \quad s-\lim _{t \rightarrow \pm \infty} \mathbf{1}_{[0,1]}\left(\frac{|\widehat{r}|}{|t|-m(|t|)}\right) \mathrm{e}^{i t H \leftarrow f=0 .}
$$

Let supp $f \subset\left[R_{1}, R_{2}\right] \times S^{2}$. We define $\widetilde{H}_{\leftarrow}=\Gamma^{1} D_{\widehat{r}}$. Using

$$
\left[\widetilde{H}_{\leftarrow}, \frac{a}{r_{+}^{2}+a^{2}} D_{\varphi}+\frac{q Q r_{+}}{r_{+}^{2}+a^{2}}\right]=0
$$

we see that it is sufficient to show

$$
s-\lim _{t \rightarrow \pm \infty} \mathbf{1}_{[0,1]}\left(\frac{|\widehat{r}|}{|t|-m(|t|)}\right) \mathrm{e}^{i t \widetilde{H}_{\leftarrow}} f=0 .
$$

We only treat the case $t \rightarrow \infty$, the case $t \rightarrow-\infty$ being analogous. We have

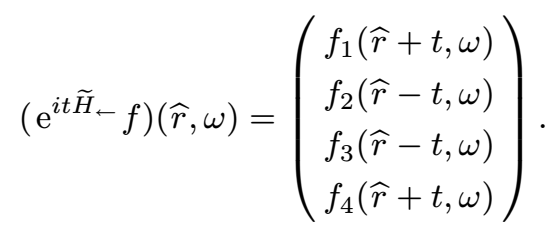

On $\operatorname{supp}\left(\mathbf{1}_{[0,1]}\left(\frac{|\widehat{r}|}{|t|-m(t)}\right) \mathrm{e}^{i t H_{\leftarrow}} f\right)_{2,3}$ we have for $t \geq-R_{1}$ :

$$
t-m(t) \geq|\widehat{r}| \geq t+R_{1}, \quad \text { which is impossible for } t \text { sufficiently large. }
$$

In the same way we find on $\operatorname{supp}\left(\mathbf{1}_{[0,1]}\left(\frac{|\widehat{r}|}{|t|-m(t)}\right) \mathrm{e}^{i t H_{\leftarrow}} f\right)_{1,4}$ for $t \geq R_{2}$ :

$$
t-m(t) \geq|\widehat{r}| \geq t-R_{2}, \quad \text { which is impossible for } t \text { sufficiently large. }
$$

Thus for $t$ sufficiently large we have

$$
\mathbf{1}_{[0,1]}\left(\frac{|\widehat{r}|}{|t|-m(t)}\right) \mathrm{e}^{i t H \leftarrow f=0 .}
$$




\subsection{Wave operators}

We shall need a characterization of the wave operators in terms of cut-off functions. Let $J \in C^{\infty}(\mathbb{R})$ such that

$$
\exists b, c \in \mathbb{R}, 0<b<c, \quad J(\widehat{r})= \begin{cases}1 & \widehat{r}<b, \\ 0 & \widehat{r}>c .\end{cases}
$$

Proposition 6.1. - We have

$$
\begin{aligned}
& W_{\rightarrow}^{ \pm}=s-\lim _{t \rightarrow \pm \infty} \mathrm{e}^{-i t H}(1-J) U_{D}(t), \\
& \Omega_{\rightarrow}^{ \pm}=s-\lim _{t \rightarrow \pm \infty} U_{D}(-t)(1-J) \mathrm{e}^{i t H}, \\
& W_{\leftarrow}^{ \pm}=s-\lim _{t \rightarrow \pm \infty} \mathrm{e}^{-i t H} J \mathrm{e}^{i t H \leftarrow}, \\
& \Omega_{\leftarrow}^{ \pm}=s-\lim _{t \rightarrow \pm \infty} \mathrm{e}^{-i t H \leftarrow J \mathrm{e}^{i t H} .}
\end{aligned}
$$

We refer to [29] for the link between the wave operators in terms of cut-off functions and the wave operators using the asymptotic velocity. The operator $\not D$ is a short range perturbation of $D_{\mathfrak{s}}$. More precisely we have the following ([15, Theorem 5.1]):

Proposition 6.2. - The wave operators

$$
\widetilde{W}_{\mathfrak{s}}^{ \pm}=s-\lim _{t \rightarrow \pm \infty} \mathrm{e}^{-i t \not D} \mathrm{e}^{i t \not D_{\mathfrak{s}}}, \quad \widetilde{\Omega}_{\mathfrak{s}}^{ \pm}=s-\lim _{t \rightarrow \pm \infty} \mathrm{e}^{-i t \not D_{\mathfrak{s}}} \mathrm{e}^{i t \not \supset}
$$

exist and we have

$$
\widetilde{\Omega}_{\mathfrak{s}}^{ \pm}=\left(\widetilde{W}_{\mathfrak{s}}^{ \pm}\right)^{*}, \quad \widetilde{W}_{\mathfrak{s}}^{ \pm} \widetilde{\Omega}_{\mathfrak{s}}^{ \pm}=\widetilde{\Omega}_{\mathfrak{s}}^{ \pm} \widetilde{W}_{\mathfrak{s}}^{ \pm}=\mathbf{1}_{\mathcal{H}_{*}} .
$$

We define $\rho^{\rho}(\mathbb{R})$ as a subspace of $C^{\infty}(\mathbb{R})$ by

$$
f \in \phi^{\rho}(\mathbb{R}) \Longleftrightarrow \forall \alpha \in \mathbb{N},\left|f^{(\alpha)}(x)\right| \leq C_{\alpha}\langle x\rangle^{\rho-\alpha}, \quad\langle x\rangle:=\sqrt{1+x^{2}} .
$$

We shall need (see [15, Corollary 3.2]):

Lemma 6.4. - (i) Let $\chi \in \phi^{0}(\mathbb{R})$. Then

$$
\left(\chi(\not)-\chi\left(\not D_{\mathfrak{s}}\right)\right)(\not \supset+i)^{-1} \quad \text { is compact. }
$$

(ii) If $\chi \in C_{0}^{\infty}(\mathbb{R})$, then

$$
\left(\not D-D_{\mathfrak{s}}\right) \chi(\not D) \text { and }\left(\not D-D_{\mathfrak{s}}\right) \chi\left(D_{\mathfrak{s}}\right) \text { are compact. }
$$

LEMMA 6.5. - The following wave operator exists:

$$
W_{0}^{ \pm}:=s-\lim _{t \rightarrow \pm \infty} \mathrm{e}^{-i t H_{0}}(1-J) \mathrm{e}^{i t H} .
$$

Furthermore we have

$$
\begin{array}{r}
W_{0}^{ \pm} \mathbf{1}_{[0, \infty)}(H)=\mathbf{1}_{[0, \infty)}\left(H_{0}\right) W_{0}^{ \pm}, \\
\forall f \in \mathcal{H}, \quad\left\|W_{0}^{ \pm} f\right\|=\left\|\mathbf{1}_{\mathbb{R}^{ \pm}}\left(P^{ \pm}\right) f\right\| .
\end{array}
$$


Proof. - We only treat the case $t \rightarrow \infty$, the case $t \rightarrow-\infty$ being analogous. By a density argument it is sufficient to show for $\chi \in C_{0}^{\infty}(\mathbb{R})$, supp $\chi \subset \mathbb{R} \backslash\{-m, m\}$ the existence of

$$
s-\lim _{t \rightarrow \infty} \mathrm{e}^{-i t H_{0}}(1-J) \mathrm{e}^{i t H} \chi(H) .
$$

Let $\epsilon_{\chi}$ as in Lemma 6.2. Let $J_{0} \in C_{0}^{\infty}(\mathbb{R}), J_{0} \geq 0$, supp $J_{0} \subset\left(\frac{1}{4} \epsilon_{\chi}, 1+2 \epsilon_{\chi}\right), J_{0}=1$ on $\left[\frac{1}{2} \epsilon_{\chi}, 1+\epsilon_{\chi}\right]$. We first show that

$$
s-\lim _{t \rightarrow \infty} \mathrm{e}^{-i t H_{0}}(1-J) \mathrm{e}^{i t H} \chi(H)=s-\lim _{t \rightarrow \infty} \mathrm{e}^{-i t H_{0}} J_{0}\left(\frac{\widehat{r}}{t}\right) \mathrm{e}^{i t H} \chi(H)
$$

if the R.H.S. exists. For this purpose let $J_{0}, J_{ \pm}$be a partition of unity with $J_{ \pm} \geq 0$, $\operatorname{supp} J_{-} \subset\left(-\infty, \frac{1}{2} \epsilon_{\chi}\right), \operatorname{supp} J_{+} \subset\left(1+\epsilon_{\chi}, \infty\right), J_{-}+J_{0}+J_{+}=1$. We have

$$
s-\lim _{t \rightarrow-\infty} \mathrm{e}^{-i t H_{0}}(1-J) J_{+}\left(\frac{\widehat{r}}{t}\right) \mathrm{e}^{i t H} \chi(H)=0
$$

by Lemma 6.1 . We write $J_{-}=J_{-}^{1}+J_{-}^{2}$, where $J_{-}^{1}, J_{-}^{2} \geq 0$ and $J_{-}^{2}$ equals one in a small neighborhood of -1 and is supported in a slightly larger neighborhood $I$ of -1 . We have

$$
s-\lim _{t \rightarrow \infty} \mathrm{e}^{-i t H_{0}}(1-J) J_{-}^{1}\left(\frac{\widehat{r}}{t}\right) \mathrm{e}^{i t H} \chi(H)=0
$$

by the maximal and minimal velocity estimates. Obviously we have for $I$ sufficiently small and $t$ sufficiently large:

$$
\mathrm{e}^{-i t H_{0}}(1-J) J_{-}^{2}\left(\frac{\widehat{r}}{t}\right) \mathrm{e}^{i t H} \chi(H)=0 .
$$

Let

Clearly

$$
J_{0}\left(\frac{\widehat{r}}{t}\right)=\mathbf{1}_{\left[\frac{1}{2} \epsilon_{\chi}, 1+\epsilon_{\chi}\right]}\left(\frac{\widehat{r}}{t}\right)+J_{0}^{1}\left(\frac{\widehat{r}}{t}\right) .
$$

$$
(1-J)(\widehat{r}) \mathbf{1}_{\left[\frac{1}{2} \epsilon_{\chi}, 1+\epsilon_{\chi}\right]}\left(\frac{\widehat{r}}{t}\right)=\mathbf{1}_{\left[\frac{1}{2} \epsilon_{\chi}, 1+\epsilon_{\chi}\right]}\left(\frac{\widehat{r}}{t}\right)
$$

for $t$ sufficiently large and

$$
s-\lim _{t \rightarrow \infty} \mathrm{e}^{-i t H_{0}}(1-J) J_{0}^{1}\left(\frac{\widehat{r}}{t}\right) \mathrm{e}^{i t H} \chi(H)=0
$$

by the maximal and minimal velocity estimates. Again by the maximal and minimal velocity estimates we obtain (6.6):

$$
s-\lim _{t \rightarrow \infty} \mathrm{e}^{-i t H_{0}} \mathbf{1}_{\left[\epsilon_{\chi}, 1+\epsilon_{\chi}\right]}\left(\frac{\widehat{r}}{t}\right) \mathrm{e}^{i t H} \chi(H)=s-\lim _{t \rightarrow \infty} \mathrm{e}^{-i t H_{0}} J_{0}\left(\frac{\widehat{r}}{t}\right) \mathrm{e}^{i t H} \chi(H) .
$$

It remains to show that the limit on the R.H.S. of (6.6) exists:

$$
\begin{aligned}
\frac{\mathrm{d}}{\mathrm{d} t} \mathrm{e}^{-i t H_{0}} J_{0}\left(\frac{\widehat{r}}{t}\right) \mathrm{e}^{i t H} \chi(H) & =\mathrm{e}^{-i t H_{0}}\left(H_{0} J_{0}\left(\frac{\widehat{r}}{t}\right)-J_{0}\left(\frac{\widehat{r}}{t}\right) H\right) \mathrm{e}^{i t H} \chi(H) \\
& =\mathrm{e}^{-i t H_{0}} J_{0}^{\prime}\left(\frac{\widehat{r}}{t}\right) \frac{1}{t} \Gamma^{1} \mathrm{e}^{i t H} \chi(H)
\end{aligned}
$$


and this last expression is integrable in $t$ by the maximal and minimal velocity estimates. Let us now prove (6.4). Let $\chi_{n} \in C_{0}^{\infty}(\mathbb{R})$ with

$$
\chi_{n}(x)= \begin{cases}1 & 0 \leq x \leq n, \\ 0 & x \leq-1 / n, x \geq n+1\end{cases}
$$

We have

$$
\chi_{n}(H)=\int \mathrm{e}^{-i t H} \widehat{\chi}_{n}(t) \mathrm{d} t
$$

where $\widehat{\chi}_{n}$ stands for the Fourier transform of $\chi_{n}$. Using $W_{0}^{ \pm} \mathrm{e}^{-i t H}=\mathrm{e}^{-i t H_{0}} W_{0}^{ \pm}$we see that

$$
W_{0}^{ \pm} \chi_{n}(H)=\chi_{n}\left(H_{0}\right) W_{0}^{ \pm}
$$

Taking the strong limit in (6.7) gives (6.4). Let us now prove (6.5). By a density argument we see that it is sufficient to establish (6.5) for $\chi(H) f, \chi \in C_{0}^{\infty}(\mathbb{R})$, supp $\chi \subset$ $\mathbb{R} \backslash\{-m, m\}$. But by $(6.6)$ we see that

$$
\begin{aligned}
\left\|W_{0}^{+} \chi(H) f\right\| & =\lim _{t \rightarrow \infty}\left\|\mathrm{e}^{-i t H_{0}} J_{0}\left(\frac{\widehat{r}}{t}\right) \mathrm{e}^{i t H} \chi(H) f\right\| \\
& =\left\|J_{0}\left(P^{+}\right) \chi(H) f\right\|=\left\|\mathbf{1}_{\mathbb{R}^{+}}\left(P^{+}\right) \chi(H) f\right\|,
\end{aligned}
$$

where we have used the minimal and maximal velocity estimates.

\subsection{Regularity results}

We first need a result on $\operatorname{supp} \Omega_{\leftarrow}^{-} f$ :

LEMma 6.6. - We have

$$
\mathbf{1}_{(-\infty, R)}(\widehat{r}) \Omega_{\leftarrow}^{-} \mathbf{1}_{[R, \infty)}(\widehat{r})=0, \quad \forall R .
$$

Proof. - We have

$$
\Omega_{\leftarrow}^{-}=s-\lim _{t \rightarrow-\infty} \mathrm{e}^{-i t H_{\leftarrow}} J \mathrm{e}^{i t H}, \quad P_{\mathcal{H}^{+}} \Omega_{\leftarrow}^{-}=\Omega_{\leftarrow}^{-} .
$$

It follows

$$
\Omega_{\leftarrow}^{-}=s-\lim _{t \rightarrow-\infty} \mathrm{e}^{-i t H_{\leftarrow}} P_{\mathcal{H}^{+}} J \mathrm{e}^{i t H} .
$$

Let $f \in \mathcal{H}$, supp $f \subset[R, \infty) \times S^{2}$. By the finite propagation speed we have for $t \leq 0$ :

$$
\text { supp e } \mathrm{e}^{i t H} f \subset[R+t, \infty) \times S^{2} .
$$

Therefore:

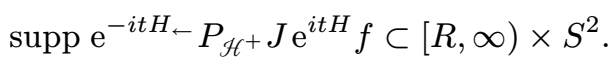

The equations (6.9), (6.10) prove the lemma.

For $\Omega_{\leftarrow}^{-}$we need the convergence in $H^{1}\left(\mathbb{R} ;\left(L^{2}\left(S^{2}\right)\right)^{4}\right)$ : 
LEMMA 6.7. - We have for all $f \in \mathcal{H}^{1}$ :

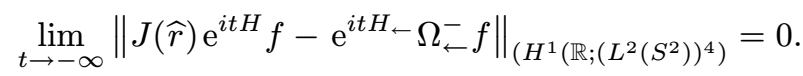

Proof. - The wave operators acting on $\mathscr{H}_{*}$ will be denoted with a tilde. By conjugation with $\mathcal{V} U$ we obtain wave operators acting on $\mathcal{H}$, e.g.

$$
\Omega_{\mathfrak{s}}^{-}=V \mathcal{U} \widetilde{\Omega}_{\mathfrak{s}}^{-} U^{*} V^{*}=s-\lim _{t \rightarrow-\infty} \mathrm{e}^{-i t H_{\mathfrak{s}}} \mathrm{e}^{i t H}
$$

with $H_{\mathfrak{s}}=\vee \mathcal{U} \boldsymbol{D}_{\mathfrak{s}} U^{*} V^{*}$. We note that

$$
\begin{gathered}
\vee \mathcal{V}: \mathcal{H}_{*}^{1} \longrightarrow \mathcal{H}^{1} ; \quad \vee \mathcal{U}: H^{1}\left(\mathbb{R}_{r_{*}} ;\left(L^{2}\left(S^{2}\right)\right)^{4}\right) \longrightarrow H^{1}\left(\mathbb{R}_{\widehat{r}} ;\left(L^{2}\left(S^{2}\right)\right)^{4}\right), \\
\mathcal{U}^{*} \mathcal{V}^{*}: \mathcal{H}^{1} \longrightarrow \mathcal{H}_{*}^{1} ; \quad \mathcal{U}^{*} \mathcal{V}^{*}: H^{1}\left(\mathbb{R}_{\widehat{r}} ;\left(L^{2}\left(S^{2}\right)\right)^{4}\right) \longrightarrow H^{1}\left(\mathbb{R}_{r_{*}} ;\left(L^{2}\left(S^{2}\right)\right)^{4}\right)
\end{gathered}
$$

are continuous. This follows from the definition of the spaces $\mathcal{H}^{1}, \mathcal{H}_{*}^{1}$ and from the estimate $0<\delta \leq k^{\prime} \leq 1$. We show:

$$
\begin{aligned}
& \forall f \in \mathscr{H}_{*}^{1}, \quad \lim _{t \rightarrow-\infty}\left\|\mathrm{e}^{i t \not D} f-\mathrm{e}^{i t D_{\mathfrak{s}}} \widetilde{\Omega}_{\mathfrak{s}}^{-} f\right\|_{\mathcal{H}_{*}^{1}}=0,
\end{aligned}
$$

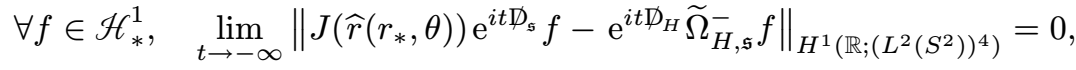

$$
\begin{aligned}
& \forall f \in H^{1}\left(\mathbb{R}_{\widehat{r}} ;\left(L^{2}\left(S^{2}\right)\right)^{4}\right) \\
& \lim _{t \rightarrow-\infty}\left\|\mathrm{e}^{i t H_{H}} \mathbf{1}_{\mathbb{R}^{+}}\left(P_{H}^{-}\right) f-\mathrm{e}^{i t H_{\leftarrow}} \Omega_{H, \leftarrow}^{-} f\right\|_{\left(H^{1}\left(\mathbb{R} ;\left(L^{2}\left(S^{2}\right)\right)^{4}\right)\right.}=0,
\end{aligned}
$$

where

$$
\begin{gathered}
\widetilde{\Omega}_{H, \mathfrak{s}}^{-}=s-\lim _{t \rightarrow-\infty} \mathrm{e}^{-i t \not_{H}} \mathrm{e}^{i t \not_{\mathfrak{s}}} \mathbf{1}_{\mathbb{R}^{+}}\left(\widetilde{P}_{\mathfrak{s}}^{-}\right), \quad \widetilde{P}_{\mathfrak{s}}^{-}=s-C_{\infty}-\lim _{t \rightarrow-\infty} \mathrm{e}^{-i t \not_{\mathfrak{s}}} \frac{r_{*}}{t} \mathrm{e}^{i t \not_{\mathfrak{s}}}, \\
H_{H}=\vee \mathcal{V} \not_{H} \mathcal{U}^{*} V^{*}, \\
\Omega_{H, \leftarrow}^{-}=s-\lim _{t \rightarrow-\infty} \mathrm{e}^{-i t \mathrm{C}_{\leftarrow}} \mathrm{e}^{i t H_{H}} \mathbf{1}_{\mathbb{R}^{+}}\left(P_{H}^{-}\right), \quad P_{H}^{-}=s-C_{\infty}-\lim _{t \rightarrow-\infty} \mathrm{e}^{-i t H_{H}} \frac{\widehat{r}}{t} \mathrm{e}^{i t H_{H}} .
\end{gathered}
$$

We refer to [14] for the existence of $\widetilde{\Omega}_{H, \mathfrak{s}}^{-}$and $\widetilde{P}_{\mathfrak{s}}^{-(1)}$. The existence of $P_{H}^{-}$follows from the existence of

$$
\widetilde{P}_{H}^{-}=s-C_{\infty}-\lim _{t \rightarrow-\infty} \mathrm{e}^{-i t \not_{H}} \frac{r_{*}}{t} \mathrm{e}^{i t \not_{H}}
$$

and the existence of $\Omega_{H, \leftarrow}^{-}$follows from the existence of $W_{c}^{ \pm}$(see proof of Theorem 4.2). Let us first argue that (6.12)-(6.14) imply (6.11). Let

$$
\Omega_{H}^{-}=s-\lim _{t \rightarrow-\infty} \mathrm{e}^{-i t H_{H}} \mathrm{e}^{i t H} \mathbf{1}_{\mathbb{R}^{+}}\left(P^{-}\right) .
$$

$\overline{\text { (1) Note that } \not D_{\mathfrak{s}}}$ can be understood as the Dirac operator in a "Reissner-Nordström type" space-time, see [15] for details. 
The existence of $\Omega_{H}^{-}$follows from the existence of $\widetilde{\Omega}_{H}^{-}$(see proof of Theorem 4.2). We have

$$
\begin{aligned}
& \left\|\left(J(\widehat{r}) \mathrm{e}^{i t H}-\mathrm{e}^{i t H \leftarrow \Omega_{\leftarrow}^{-}}\right) f\right\|_{H^{1}\left(\mathbb{R} ;\left(L^{2}\left(S^{2}\right)\right)^{4}\right)} \\
& \leq\left\|J(\widehat{r})\left(\mathrm{e}^{i t H}-\mathrm{e}^{i t H_{\mathfrak{s}}} \Omega_{\mathfrak{s}}^{-}\right) f\right\|_{H^{1}\left(\mathbb{R} ;\left(L^{2}\left(S^{2}\right)\right)^{4}\right)} \\
& \quad+\left\|\left(J(\widehat{r}) \mathrm{e}^{i t H_{\mathfrak{s}}} \Omega_{\mathfrak{s}}^{-}-\mathrm{e}^{i t H_{H}} \Omega_{H}^{-}\right) f\right\|_{H^{1}\left(\mathbb{R} ;\left(L^{2}\left(S^{2}\right)\right)^{4}\right)} \\
& \quad+\left\|\left(\mathrm{e}^{i t H_{H}} \Omega_{H}^{-}-\mathrm{e}^{i t H_{\leftarrow}} \Omega_{\leftarrow}^{-}\right) f\right\|_{H^{1}\left(\mathbb{R} ;\left(L^{2}\left(S^{2}\right)\right)^{4}\right)}=: I_{1}+I_{2}+I_{3} .
\end{aligned}
$$

We first estimate $I_{1}$. We have, thanks to (4.41) and (6.12):

$$
\begin{aligned}
I_{1} & \leq\left\|\left(\mathrm{e}^{i t \not D}-\mathrm{e}^{i t \not_{\mathfrak{s}} \widetilde{\Omega}_{\mathfrak{s}}^{-}}\right) V^{*} \mathcal{U}^{*} f\right\|_{H^{1}\left(\mathbb{R} ;\left(L^{2}\left(S^{2}\right)\right)^{4}\right)} \\
& \lesssim\left\|\left(\mathrm{e}^{i t \not D}-\mathrm{e}^{i t \not_{\mathfrak{s}} \widetilde{\Omega}_{\mathfrak{s}}^{-}}\right) V^{*} \mathcal{U}^{*} f\right\|_{\mathcal{H}_{*}^{1}} \longrightarrow 0, \quad t \rightarrow-\infty .
\end{aligned}
$$

In order to estimate $I_{2}$ we observe that $\widetilde{\Omega}_{\mathfrak{s}}^{-}: \mathcal{H}_{*}^{1} \rightarrow \mathcal{H}_{*}^{1}$ and that $\widetilde{\Omega}_{H}^{-}\left(\widetilde{\Omega}_{\mathfrak{s}}^{-}\right)^{*}=\widetilde{\Omega}_{H, \mathfrak{s}}^{-}$. We obtain using (6.13):

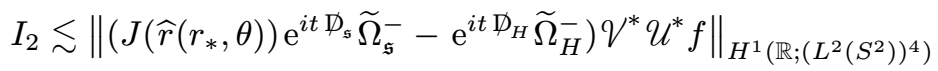

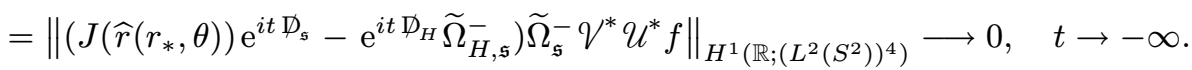

We now estimate $I_{3}$. We observe that $D\left(H_{H}\right)=H^{1}\left(\mathbb{R} ;\left(L^{2}\left(S^{2}\right)\right)^{4}\right)$ and that the graph norm of $H_{H}$ is equivalent to the norm of $H^{1}\left(\mathbb{R} ;\left(L^{2}\left(S^{2}\right)\right)^{4}\right)$. This entails $\Omega_{H}^{-} f \in$ $H^{1}\left(\mathbb{R} ;\left(L^{2}\left(S^{2}\right)\right)^{4}\right)$. Observe also that

$$
\Omega_{\leftarrow}^{-}=\Omega_{H, \leftarrow}^{-} \Omega_{H}^{-} .
$$

We obtain using (6.14):

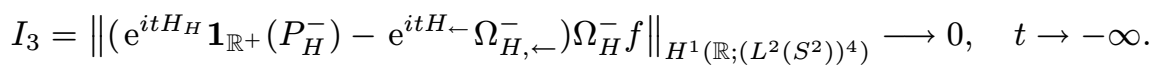

The proof of (6.13) is analogous to the proof of [37, Lemma 6.3] and we therefore omit it.

Let us show (6.12). Using the uniform estimates

$$
\left\|\mathrm{e}^{i t \not D} f\right\|_{\mathscr{H}_{*}^{1}} \lesssim\|f\|_{\mathscr{H}_{*}^{1}} \quad \text { and } \quad\left\|\mathrm{e}^{i t D_{s}} f\right\|_{\mathcal{H}_{*}^{1}} \lesssim\|f\|_{\mathcal{H}_{*}^{1}}
$$

we argue that we can replace $f$ by $\chi(\not D) f, \chi \in C_{0}^{\infty}(\mathbb{R})$. We then write

$$
\left.\not D\left(\mathrm{e}^{i t \not D}-\mathrm{e}^{i t \not_{\mathfrak{s}}} \widetilde{\Omega}_{\mathfrak{s}}^{-}\right) \chi(\not D) f=\left(\mathrm{e}^{i t \not D}-\mathrm{e}^{i t \not_{\mathfrak{s}}} \widetilde{\Omega}_{\mathfrak{s}}^{-}\right) \not D \chi(\not D) f+\left(\not D_{\mathfrak{s}}-\not D\right) \chi(\not D)_{\mathfrak{s}}\right) \mathrm{e}^{i t \not_{\mathfrak{s}}} \widetilde{\Omega}_{\mathfrak{s}}^{-} f .
$$

When $t \rightarrow-\infty$ the first term goes to zero by Proposition 6.2 and the second term goes to zero because $\left(\not_{\mathfrak{s}}-\not D\right) \chi\left(D_{\mathfrak{s}}\right)$ is compact by Lemma 6.4 (ii).

Let us show (6.14). By a density argument we can replace $f$ by $\chi\left(H_{H}\right) f$ with $\chi \in C_{0}^{\infty}(\mathbb{R})$. Then we have

$$
\begin{aligned}
& H_{\leftarrow}\left(\mathrm{e}^{i t H_{H}} \mathbf{1}_{\mathbb{R}^{+}}\left(P_{H}^{-}\right)\right.\left.-\mathrm{e}^{i t H_{\leftarrow}} \Omega_{H, \leftarrow}^{-}\right) \chi\left(H_{H}\right) f \\
&=\left(H_{\leftarrow}-H_{H}\right) \chi\left(H_{H}\right) \mathrm{e}^{i t H_{H}} \mathbf{1}_{\mathbb{R}^{+}}\left(P_{H}^{-}\right) f \\
& \quad+\left(\mathrm{e}^{i t H_{H}} \mathbf{1}_{\mathbb{R}^{+}}\left(P_{H}^{-}\right)-\mathrm{e}^{i t H_{\leftarrow}} \Omega_{H, \leftarrow}^{-}\right) H_{H} \chi\left(H_{H}\right) f .
\end{aligned}
$$


The second term in (6.15) goes to zero by definition of $\Omega_{H, \leftarrow}^{-}$. In order to show that the first term in (6.15) goes to zero it is sufficient to show

$$
\left(\not D_{\leftarrow}-\not D_{H}\right) \chi\left(\not D_{H}\right) \mathrm{e}^{i t \not_{H}} \mathbf{1}_{\mathbb{R}^{+}}\left(\widetilde{P}_{H}^{-}\right) f \rightarrow 0, \quad t \rightarrow-\infty .
$$

We have

$$
\begin{aligned}
\|\left(\not D_{\leftarrow}-\not D_{H}\right) \chi & \left(\not D_{H}\right) \mathrm{e}^{i t \not D_{H}} \mathbf{1}_{\mathbb{R}^{+}}\left(\widetilde{P}_{H}^{-}\right) f \|_{\mathcal{H}_{*}} \\
& \leq\left\|m\left(r_{*}\right) \not_{H} \chi\left(\not_{H}\right) \mathrm{e}^{i t \not_{H}} \mathbf{1}_{\mathbb{R}^{+}}\left(\widetilde{P}_{H}^{-}\right) f\right\|_{\mathcal{H}_{*}}
\end{aligned}
$$

with $m\left(r_{*}\right) \rightarrow 0,\left|r_{*}\right| \rightarrow \infty$. We then use the spherical symmetry of the expression on the R.H.S. of (6.16) and the fact that $m\left(r_{*}\right) \mathbb{D}_{H}^{n \ell} \chi\left(\mathbb{D}_{H}^{n \ell}\right)$ is compact on $\mathcal{H}_{*}^{n \ell}$ to conclude that the R.H.S. of (6.16) goes to zero when $t \rightarrow-\infty$. 



\section{CHAPTER 7}

\section{THE CHARACTERISTIC CAUCHY PROBLEM}

The aim of this chapter is to solve a characteristic Cauchy problem in a space-time region near the collapsing star. The results of this chapter will be used later in the proof of the main theorem. We start by studying a characteristic Cauchy problem for the Dirac equation in the whole exterior Kerr-Newman space-time. In Section 7.1 we formulate the main result of this chapter. Section 7.2 is devoted to the usual Cauchy problem with data on a lipschitz space-like surface. The main theorem is proven in Section 7.3. In Section 7.4 we use these results to solve the characteristic Cauchy problem near the collapsing star. Our strategy is similar to that of Hörmander in [32] for the characteristic Cauchy problem for the wave equation (see also [41] for weaker assumptions on the metric). A characteristic Cauchy problem for the Dirac equation has been considered in [35] in a somewhat different setting.

\subsection{Main results}

Let (see Figure 1):

$$
\begin{aligned}
\Lambda_{T}^{ \pm} & :=\left\{( \pm \widehat{r}, \widehat{r}, \omega) ; 0 \leq \pm \widehat{r} \leq T, \omega \in S^{2}\right\}, \quad \Lambda_{T}:=\Lambda_{T}^{+} \cup \Lambda_{T}^{-}, \\
K_{T} & :=\left\{(t, \widehat{r}, \omega) ;|\widehat{r}| \leq T,|\widehat{r}| \leq t \leq T, \omega \in S^{2}\right\}, \\
\Sigma_{T}:= & =\left\{(T, \widehat{r}, \omega) ;|\widehat{r}| \leq T, \omega \in S^{2}\right\} .
\end{aligned}
$$

We need the spaces

$$
\begin{aligned}
\mathcal{H}_{T} & :=L^{2}\left(\left([-T, T] \times S^{2}, \mathrm{~d} \widehat{r} \mathrm{~d} \omega\right) ; \mathbb{C}^{4}\right), \\
\mathcal{H}_{T}^{1} & :=\left\{u \in \mathcal{H}_{T} ; H u \in \mathcal{H}_{T}\right\}, \quad\|u\|_{\mathcal{H}_{T}^{1}}^{2}=\|u\|_{\mathcal{H}_{T}}^{2}+\|H u\|_{\mathcal{H}_{T}}^{2}, \\
L_{T,-}^{2} & :=L^{2}\left(\left([-T, 0] \times S^{2}, \mathrm{~d} \widehat{r} \mathrm{~d} \omega\right) ; \mathbb{C}^{2}\right), \\
L_{T,+}^{2} & :=L^{2}\left(\left([0, T] \times S^{2}, \mathrm{~d} \widehat{r} \mathrm{~d} \omega\right) ; \mathbb{C}^{2}\right) .
\end{aligned}
$$

Let $\Phi_{T} \in C^{\infty}\left(\Sigma_{T} ; \mathbb{S}_{A} \oplus \mathbb{S}^{A^{\prime}}\right)$. By the usual theorems for hyperbolic equations we can associate to $\Phi_{T}$ a smooth solution $\phi_{A} \oplus \chi^{A^{\prime}} \in C^{\infty}\left(K_{T} ; \mathbb{S}_{A} \oplus \mathbb{S}^{A^{\prime}}\right)$ (see [40] for details). 


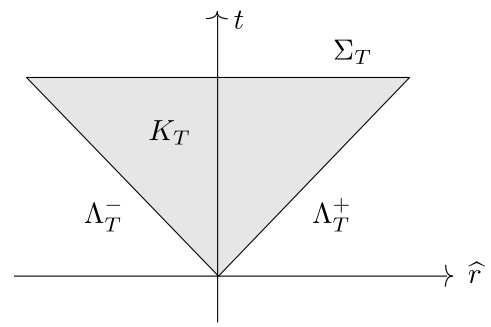

Figure 1. The characteristic Cauchy problem

We use the $\ell^{a}, n^{a}, m^{a}$ tetrad and the $(t, \widehat{r}, \omega)$ coordinate system. Let

$$
\Psi=\sqrt[4]{\frac{\rho^{2} \Delta \sigma^{2}}{\left(r^{2}+a^{2}\right)^{2} k^{\prime 2}}}\left(\phi_{0}, \phi_{1}, \chi_{1^{\prime}},-\chi_{0^{\prime}}\right)
$$

be the associated density spinor. The spinor fields $o^{A}$ and $\iota^{A}$ are smooth and non vanishing on $\Lambda_{T}^{ \pm}$, therefore we can associate to this solution the smooth trace of $\Psi$ :

$$
\begin{aligned}
\mathcal{J}: \Phi_{T} \longmapsto & \left(\Psi_{2}, \Psi_{3}\right)(-\widehat{r}, \widehat{r}, \omega) \oplus\left(\Psi_{1}, \Psi_{4}\right)(\widehat{r}, \widehat{r}, \omega) \\
& \in C^{\infty}\left([-T, 0] \times S^{2} ; \mathbb{C}^{2}\right) \oplus C^{\infty}\left([0, T] \times S^{2} ; \mathbb{C}^{2}\right) .
\end{aligned}
$$

Using the conserved current we obtain by Stokes' theorem

$$
\int_{\Sigma_{T}} *\left(\phi_{A} \bar{\phi}_{A^{\prime}} \mathrm{d} x^{A A^{\prime}}+\bar{\chi}_{A} \chi_{A^{\prime}} \mathrm{d} x^{A A^{\prime}}\right)=\int_{\Lambda_{T}} *\left(\phi_{A} \bar{\phi}_{A^{\prime}} \mathrm{d} x^{A A^{\prime}}+\bar{\chi}_{A} \chi_{A^{\prime}} \mathrm{d} x^{A A^{\prime}}\right) .
$$

Let $\varphi(\widehat{r})=|\widehat{r}|$. The normal to $\Lambda_{T}^{-}$is $n^{a}$, the normal to $\Lambda_{T}^{+}$is $\ell^{a}$. We compute:

$$
\left.\left.\left(\ell^{a} \partial_{a}\right\lrcorner \mathrm{d} \Omega\right)\left.\right|_{\Lambda_{T}^{+}}=\left(n^{a} \partial_{a}\right\lrcorner \mathrm{d} \Omega\right)\left.\right|_{\Lambda_{T}^{-}}=\sqrt{\frac{2 \rho^{2} \Delta \sigma^{2}}{\left(r^{2}+a^{2}\right)^{2} k^{\prime 2}}} \mathrm{~d} \widehat{r} \wedge \mathrm{d} \omega .
$$

Following (4.15) we find:

$$
\begin{aligned}
& \int_{\Lambda_{T}} *\left(\phi_{A} \bar{\phi}_{A^{\prime}} \mathrm{d} x^{A A^{\prime}}+\bar{\chi}_{A} \chi_{A^{\prime}} \mathrm{d} x^{A A^{\prime}}\right) \\
&=\sqrt{2} \int_{-T}^{0} \int_{S^{2}}\left(\left|\Psi_{2}\right|^{2}+\left|\Psi_{3}\right|^{2}\right)(-\widehat{r}, \widehat{r}, \omega) \mathrm{d} \widehat{r} \mathrm{~d} \omega \\
& \quad+\sqrt{2} \int_{0}^{T} \int_{S^{2}}\left(\left|\Psi_{1}\right|^{2}+\left|\Psi_{4}\right|^{2}\right)(\widehat{r}, \widehat{r}, \omega) \mathrm{d} \widehat{r} \mathrm{~d} \omega
\end{aligned}
$$


where $\Psi=\left(\Psi_{1}, \Psi_{2}, \Psi_{3}, \Psi_{4}\right)$. It follows:

$$
\begin{aligned}
& \int_{\Sigma_{T}} *\left(\phi_{A} \bar{\phi}_{A^{\prime}} \mathrm{d} x^{A A^{\prime}}+\bar{\chi}_{A} \chi_{A^{\prime}} \mathrm{d} x^{A A^{\prime}}\right) \\
&=\sqrt{2} \int_{-T}^{0} \int_{S^{2}}\left(\left|\Psi_{2}\right|^{2}+\left|\Psi_{3}\right|^{2}\right)(-\widehat{r}, \widehat{r}, \omega) \mathrm{d} \widehat{r} \mathrm{~d} \omega \\
& \quad+\sqrt{2} \int_{0}^{T} \int_{S^{2}}\left(\left|\Psi_{1}\right|^{2}+\left|\Psi_{4}\right|^{2}\right)(\widehat{r}, \widehat{r}, \omega) \mathrm{d} \widehat{r} \mathrm{~d} \omega .
\end{aligned}
$$

Therefore the operator $\mathcal{T}$ possesses an extension to a bounded operator

$$
\mathcal{J} \in \mathscr{L}\left(L^{2}\left(\Sigma_{T} ; \mathbb{S}_{A} \oplus \mathbb{S}^{A^{\prime}}\right) ; L^{2}\left([-T, 0] \times S^{2} ; \mathbb{C}^{2}\right) \oplus L^{2}\left([0, T] \times S^{2} ; \mathbb{C}^{2}\right)\right) .
$$

Our first result is:

THEOREM 7.1. $-\frac{1}{\sqrt[4]{2}} \mathcal{T}$ is an isometry.

The proof of this theorem will be given in Section 7.3.

The characteristic data $\mathcal{T}\left(\Phi_{T}\right)$ contains information only about $\phi_{1}, \chi_{1^{\prime}}$ on $\Lambda_{T}^{-}$and $\phi_{0}, \chi_{0^{\prime}}$ on $\Lambda_{T}^{+}$. The functions $\phi_{0}, \chi_{0^{\prime}}$ on $\Lambda_{T}^{-}$resp. $\phi_{1}, \chi_{1^{\prime}}$ on $\Lambda_{T}^{+}$are obtained from the given data by restriction of the equation to $\Lambda_{T}^{ \pm}$. On $\Lambda_{T}^{-}$we have (see (4.8)):

$$
\begin{aligned}
& n^{\boldsymbol{a}}\left(\partial_{\boldsymbol{a}}-i q \Phi_{\boldsymbol{a}}\right) \phi_{0}-m^{\boldsymbol{a}}\left(\partial_{\boldsymbol{a}}-i q \Phi_{\boldsymbol{a}}\right) \phi_{1}+(\mu-\gamma) \phi_{0}+(\tau-\beta) \phi_{1}=\frac{m}{\sqrt{2}} \chi_{1^{\prime}}, \\
& n^{\boldsymbol{a}}\left(\partial_{\boldsymbol{a}}-i q \Phi_{\boldsymbol{a}}\right) \chi_{0^{\prime}}-\bar{m}^{\boldsymbol{a}}\left(\partial_{\boldsymbol{a}}-i q \Phi_{\boldsymbol{a}}\right) \chi_{1^{\prime}}+(\bar{\mu}-\bar{\gamma}) \chi_{0^{\prime}}+(\bar{\tau}-\bar{\beta}) \chi_{1^{\prime}}=\frac{m}{\sqrt{2}} \phi_{1},
\end{aligned}
$$

where $\phi_{1}, \chi_{1^{\prime}}$ have to be considered as source terms. Putting $g(\widehat{r}, \omega)=\Psi(-\widehat{r}, \widehat{r}, \omega)$, $\widehat{g}(\widehat{r}, \omega)=\Psi(\widehat{r}, \widehat{r}, \omega)$ we find

$$
\begin{gathered}
-D_{\widehat{r}} g_{1,4}=\left(\left(P_{\omega}+W\right) g\right)_{1,4}, \quad g_{1,4}(0, \omega)=\widehat{g}_{1,4}(0, \omega), \\
D_{\widehat{r} \widehat{g}_{2,3}}=\left(\left(P_{\omega}+W\right) \widehat{g}\right)_{2,3}, \quad \widehat{g}_{2,3}(0, \omega)=g_{2,3}(0, \omega) .
\end{gathered}
$$

Here $P_{\omega}, W$ are defined in (4.39), (4.40). We understand $\widehat{r}$ as a time parameter which goes from 0 to $-T$ for (7.4) and from 0 to $T$ for (7.5). We write (7.4) as

$$
\partial_{\widehat{r}} g_{1,4}=i A(\widehat{r}) g_{1,4}+S(\widehat{r}), \quad g_{1,4}(0, \omega)=\widehat{g}_{1,4}(0, \omega)
$$

with

$$
\begin{aligned}
& A(\widehat{r})=-\left\{\frac{m_{\theta}^{1}}{2}+\left(\begin{array}{ll}
W_{11} & W_{14} \\
W_{41} & W_{44}
\end{array}\right)\right\}, \\
& S(\widehat{r})=-\left\{\left\{\frac{m_{\theta}^{2}}{2}+\frac{a_{0} h^{2}}{\sin \theta} D_{\varphi}\left(\begin{array}{rr}
-1 & 0 \\
0 & 1
\end{array}\right)+\left(\begin{array}{ll}
W_{12} & W_{13} \\
W_{42} & W_{43}
\end{array}\right)\right\}\left(\begin{array}{l}
g_{2} \\
g_{3}
\end{array}\right)\right\} .
\end{aligned}
$$


We want to show that (7.6) has a unique solution and to this purpose we must analyze $A(\widehat{r})$. We have

$$
\frac{m_{\theta}^{1}}{2}=\frac{1}{2} \frac{\beta h \sqrt{a_{0}}}{\sqrt{\alpha+1}}\left(D_{\theta}+\frac{\cot \theta}{2 i}\right) \sqrt{a_{0}} h \sqrt{\alpha+1}+h c .
$$

We want to show that the operator

$$
\mathrm{d}_{\theta}:=D_{\theta}+\frac{\cot \theta}{2 i}
$$

is selfadjoint with some suitable domain. To this purpose we introduce the unitary transformation

$$
U: L^{2}\left(S^{2}, \mathrm{~d} \omega\right) \longrightarrow L^{2}\left(S^{2}, \mathrm{~d} \theta \mathrm{d} \varphi\right), \quad u(\theta, \varphi) \longmapsto u(\theta, \varphi) \sqrt{\sin \theta} .
$$

Clearly

$$
\widehat{\mathrm{d}}_{\theta}=U \mathrm{~d}_{\theta} U^{*}=D_{\theta},
$$

which is selfadjoint on $L^{2}\left(S^{2}, \mathrm{~d} \theta \mathrm{d} \varphi\right)$ with domain

$$
D\left(D_{\theta}\right)=\left\{u \in L^{2}\left(S^{2}, \mathrm{~d} \theta \mathrm{d} \varphi\right) ; D_{\theta} u \in L^{2}\left(S^{2}, \mathrm{~d} \theta \mathrm{d} \varphi\right), u(0, .)=u(\pi, .)\right\} .
$$

Then $\mathrm{d}_{\theta}$ is selfadjoint with domain $D\left(\mathrm{~d}_{\theta}\right)=U^{*} D\left(D_{\theta}\right)$. It is easy to check that $\widehat{r} \mapsto A(\widehat{r}) v$ is continuously differentiable for $v \in Y:=\left(D\left(\mathrm{~d}_{\theta}\right)\right)^{2}$. We can apply [43, Theorem 5.4.8] and associate a unitary evolution system $V\left(\widehat{r}, \widehat{r}^{\prime}\right)$. For smooth $g_{2,3}, \widehat{g}_{1,4}$ we have $S(\widehat{r}) \in C([-T, 0] ; Y)$ and $\widehat{g}(0, \omega) \in Y$. By [43, Theorem 5.5.2] (7.6) possesses a unique $Y$-valued solution given by

$$
g_{1,4}(\widehat{r}, \omega)=V(\widehat{r}, 0) \widehat{g}_{1,4}(0, \omega)+\int_{0}^{\widehat{r}} V\left(\widehat{r}, \widehat{r}^{\prime}\right) S\left(\widehat{r}^{\prime}\right) \mathrm{d} \widehat{r}^{\prime} .
$$

For given $g_{2,3}, \widehat{g}_{1,4}$ we define $g_{1,4}, \widehat{g}_{2,3}$ as the solutions of the partial differential equations (7.4), (7.5) and put

$$
\begin{aligned}
& g_{2,3}^{H}(\widehat{r}, \omega):=\frac{1}{2}\left(-D_{\widehat{r}} g_{2,3}+\left(\left(P_{\omega}+W\right)_{g}\right)_{2,3}\right), \\
& \widehat{g}_{1,4}^{H}(\widehat{r}, \omega):=\frac{1}{2}\left(D_{\widehat{r}} \widehat{g}_{1,4}+\left(\left(P_{\omega}+W\right) \widehat{g}\right)_{1,4}\right) .
\end{aligned}
$$

As $g_{1,4}$ is a $Y$-valued solution we have (the argument for $\widehat{g}_{2,3}$ is analogous):

$$
g_{2,3}^{H} \in L^{2}\left(\left([-T, 0] \times S^{2}, \mathrm{~d} \widehat{r} \mathrm{~d} \omega\right) ; \mathbb{C}^{2}\right), \quad \widehat{g}_{1,4}^{H} \in L^{2}\left(\left([0, T] \times S^{2}, \mathrm{~d} \widehat{r} \mathrm{~d} \omega\right) ; \mathbb{C}^{2}\right) .
$$

We define $\widetilde{H}^{1}$ as the completion of $C^{\infty}\left([-T, 0] \times S^{2} ; \mathbb{C}^{2}\right) \oplus C^{\infty}\left([0, T] \times S^{2} ; \mathbb{C}^{2}\right)$ in the norm

$$
\left\|\left(g_{2,3}, \widehat{g}_{1,4}\right)\right\|_{\widetilde{H}^{1}}^{2}=2\left(\left\|g_{2,3}\right\|_{L_{T,-}^{2}}^{2}+\left\|\widehat{g}_{1,4}\right\|_{L_{T,+}^{2}}^{2}+\left\|g_{2,3}^{H}\right\|_{L_{T,-}^{2}}^{2}+\left\|\widehat{g}_{1,4}^{H}\right\|_{L_{T,+}^{2}}^{2}\right) \text {. }
$$

We now start with $\Psi_{T} \in C^{\infty}\left(\Sigma_{T} ; \mathbb{C}^{4}\right)$. Then we can associate a classical solution $\Psi \in C^{\infty}\left(K_{T} ; \mathbb{C}^{4}\right)$ and the traces

$g_{2,3}(\widehat{r}, \omega)=\Psi_{2,3}(-\widehat{r}, \widehat{r}, \omega),-T \leq \widehat{r} \leq 0 \quad$ and $\quad \widehat{g}_{1,4}(\widehat{r}, \omega)=\Psi_{1,4}(\widehat{r}, \widehat{r}, \omega), 0 \leq \widehat{r} \leq T$ are well defined. By the previous discussion

$g_{1,4}(\widehat{r}, \omega)=\Psi_{1,4}(-\widehat{r}, \widehat{r}, \omega),-T \leq \widehat{r} \leq 0 \quad$ and $\quad \widehat{g}_{2,3}(\widehat{r}, \omega)=\Psi_{2,3}(\widehat{r}, \widehat{r}, \omega), 0 \leq \widehat{r} \leq T$ 
are solutions of equations (7.4), (7.5). As $\Psi$ is a solution of the Dirac equation, so is $H \Psi \in C^{\infty}\left(K_{T} ; \mathbb{C}^{4}\right)$. We want to calculate $\left.H \Psi\right|_{\Lambda_{T}^{ \pm}}$in terms of $g_{2,3}, \widehat{g}_{1,4}$ and to this purpose we introduce characteristic coordinates

$$
X=t-\widehat{r}, T=t+\widehat{r} \Longleftrightarrow t=\frac{1}{2}(X+T), \widehat{r}=\frac{1}{2}(T-X) .
$$

Then we have

$$
\begin{aligned}
(H \Psi)_{2,3} & =\left(D_{X}-D_{T}\right) \Psi_{2,3}+\left(\left(P_{\omega}+W\right) \Psi\right)_{2,3}=D_{X} \Psi_{2,3}+\frac{1}{2}\left(\left(P_{\omega}+W\right) \Psi\right)_{2,3} \\
& \Rightarrow(H \Psi)_{2,3}(-\widehat{r}, \widehat{r}, \omega)=\frac{1}{2}\left(-D_{\widehat{r}} g_{2,3}+\left(\left(P_{\omega}+W\right) g\right)_{2,3}\right) .
\end{aligned}
$$

In a similar manner we find

$$
(H \Psi)_{1,4}(\widehat{r}, \widehat{r}, \omega)=\frac{1}{2}\left(D_{\widehat{r}} \widehat{g}_{1,4}+\left(\left(P_{\omega}+W\right) \widehat{g}\right)_{1,4}\right) .
$$

Using the identity (7.3) we find

$$
\left\|H \Psi_{T}\right\|_{\mathcal{H}_{T}}^{2}=2\left(\left\|g_{2,3}^{H}\right\|_{L_{T,-}^{2}}^{2}+\left\|\widehat{g}_{1,4}^{H}\right\|_{L_{T,+}^{2}}^{2}\right)
$$

and therefore

$$
\left\|\Psi_{T}\right\|_{\mathcal{H}_{T}^{1}}^{2}=\left\|\left(g_{2,3}, \widehat{g}_{1,4}\right)\right\|_{\widetilde{H}^{1}}^{2} .
$$

This means that the trace operator

$$
\begin{aligned}
\mathcal{T}: & C^{\infty}\left(\Sigma_{T} ; \mathbb{C}^{4}\right) \longrightarrow C^{\infty}\left([-T, 0] \times S^{2} ; \mathbb{C}^{2}\right) \oplus C^{\infty}\left([0, T] \times S^{2} ; \mathbb{C}^{2}\right), \\
\Psi_{T} \longmapsto & \left(\Psi_{2,3}(-\widehat{r}, \widehat{r}, \omega), \Psi_{1,4}(\widehat{r}, \widehat{r}, \omega)\right)
\end{aligned}
$$

extends to a bounded operator $\mathcal{T}_{H} \in \mathscr{L}\left(\mathcal{H}_{T}^{1} ; \widetilde{H}^{1}\right)$.

Our second result is

THEOREM 7.2. $-\mathcal{J}_{H}$ is an isometry.

The proof of this theorem will be given in Section 7.3.

REMARK 7.1. - If $\Psi_{T} \in \mathcal{H}_{T}^{1}$, then the trace $\left(\Psi_{2,3}(-\widehat{r}, \widehat{r}, \omega), \Psi_{1,4}(\widehat{r}, \widehat{r}, \omega)\right)$ exists in the usual sense and it is in $\widetilde{H}^{1}$ by (7.11). This means that the operator $\mathcal{J}_{H}$ is defined as the usual trace and that $\mathcal{T}$ is an extension of $\mathcal{T}_{H}$.

\subsection{The Cauchy problem with data on a lipschitz space-like hypersurface}

Let $\varphi:\left[-T_{\varphi}, T_{\varphi}\right] \rightarrow \mathbb{R}$ be lipschitz continuous, $T_{\varphi} \geq T$,

$$
\left|\varphi^{\prime}(\widehat{r})\right| \leq \widetilde{\alpha}<1 \quad \text { a.e. }
$$


Thanks to (7.12) the hypersurface $\Lambda_{\varphi}:=\left\{(\varphi(\widehat{r}), \widehat{r}, \omega) ;|\widehat{r}| \leq T_{\varphi}, \omega \in S^{2}\right\}$ is space-like. Indeed we have

$$
\begin{array}{r}
g\left(\varphi^{\prime}(\widehat{r}) \partial_{t}+\partial_{\widehat{r}}, \varphi^{\prime}(\widehat{r}) \partial_{t}+\partial_{\widehat{r}}\right)=\left(1+\frac{Q^{2}-2 M r}{\rho^{2}}\right) \varphi^{\prime 2}-\frac{\rho^{2} \Delta}{\left(r^{2}+a^{2}\right)^{2} k^{2}} \\
<\left(1+\frac{Q^{2}-2 M r}{\rho^{2}}\right)-\frac{\rho^{2} \Delta}{\sigma^{2}} \\
=-\frac{a^{2} \sin ^{2} \theta\left(2 M r-Q^{2}\right)^{2}}{\rho^{2} \sigma^{2}} \leq 0, \\
g\left(\partial_{\varphi}, \partial_{\varphi}\right)<0, \quad g\left(\partial_{\theta}, \partial_{\theta}\right)<0 .
\end{array}
$$

Let for $0 \leq t \leq T$

$$
\begin{aligned}
\Sigma_{t}^{\varphi} & :=\left\{(t, \widehat{r}, \omega) \in\{t\} \times\left[-T_{\varphi}, T_{\varphi}\right] \times S^{2} ; t>\varphi(\widehat{r})\right\}, \\
K_{T}^{\varphi} & :=\bigcup_{0 \leq t \leq T} \Sigma_{t}^{\varphi}, \\
\mathcal{R}_{t}^{\varphi} & :=\left\{\widehat{r} \in\left(-T_{\varphi}, T_{\varphi}\right) ; t>\varphi(\widehat{r})\right\} .
\end{aligned}
$$

We will suppose $\mathcal{R}_{T}^{\varphi}=\left(-T_{\varphi}, T_{\varphi}\right), \varphi\left(-T_{\varphi}\right)=\varphi\left(T_{\varphi}\right)=T$. We also define the spaces

$$
\begin{aligned}
& \mathcal{H}_{t, \varphi}=L^{2}\left(\left(\Sigma_{t}^{\varphi}, \mathrm{d} \widehat{r} \mathrm{~d} \omega\right) ; \mathbb{C}^{4}\right), \\
& \mathscr{H}_{t, \varphi}^{1}=\left\{u \in \mathscr{H}_{t, \varphi} ; H u \in \mathscr{H}_{t, \varphi}\right\}, \quad\|u\|_{\mathcal{H}_{t, \varphi}^{1}}^{2}=\|u\|_{\mathcal{H}_{t, \varphi}}^{2}+\|H u\|_{\mathcal{H}_{t, \varphi}}^{2} .
\end{aligned}
$$

The aim of this section is to solve the Cauchy problem

$$
\left\{\begin{array}{l}
\partial_{t} \Psi=i H \Psi, \quad(t, \widehat{r}, \omega) \in K_{T}^{\varphi}, \\
\Psi(\varphi(\widehat{r}), \widehat{r}, \omega)=g(\widehat{r}, \omega), \quad(\widehat{r}, \omega) \in\left[-T_{\varphi}, T_{\varphi}\right] \times S^{2} .
\end{array}\right.
$$

We first define the space of data. For $g \in C^{\infty}\left(\left[-T_{\varphi}, T_{\varphi}\right] ; \mathbb{C}^{4}\right)$ we define

$$
\begin{aligned}
g_{\varphi}^{H}(\widehat{r}, \omega):=( & \left.\operatorname{Diag}\left(\frac{1}{1+\varphi^{\prime}},-\frac{1}{1-\varphi^{\prime}},-\frac{1}{1-\varphi^{\prime}}, \frac{1}{1+\varphi^{\prime}}\right) D_{\widehat{r}} g\right)(\widehat{r}, \omega) \\
+ & \left(\operatorname{Diag}\left(\frac{1}{1+\varphi^{\prime}}, \frac{1}{1-\varphi^{\prime}}, \frac{1}{1-\varphi^{\prime}}, \frac{1}{1+\varphi^{\prime}}\right)\left(P_{\omega}+W\right) g\right)(\widehat{r}, \omega) .
\end{aligned}
$$

We define $\widetilde{H}_{\varphi}^{1}$ as the completion of $C^{\infty}\left(\left[-T_{\varphi}, T_{\varphi}\right] \times S^{2} ; \mathbb{C}^{4}\right)$ in the norm

$$
\begin{aligned}
\|g\|_{\widetilde{H}_{\varphi}^{1}}^{2}:=\left\|\left(1-\varphi^{\prime}\right)^{\frac{1}{2}} g_{2,3}\right\|_{L^{2}\left(\left[-T_{\varphi}, T_{\varphi}\right] \times S^{2} ; \mathbb{C}^{2}\right)}^{2} & \\
+ & \left\|\left(1-\varphi^{\prime}\right)^{\frac{1}{2}}\left(g_{\varphi}^{H}\right)_{2,3}\right\|_{L^{2}\left(\left[-T_{\varphi}, T_{\varphi}\right] \times S^{2} ; \mathbb{C}^{2}\right)}^{2} \\
& +\left\|\left(1+\varphi^{\prime}\right)^{\frac{1}{2}} g_{1,4}\right\|_{L^{2}\left(\left[-T_{\varphi}, T_{\varphi}\right] \times S^{2} ; \mathbb{C}^{2}\right)}^{2} \\
& +\left\|\left(1+\varphi^{\prime}\right)^{\frac{1}{2}}\left(g_{\varphi}^{H}\right)_{1,4}\right\|_{L^{2}\left(\left[-T_{\varphi}, T_{\varphi}\right] \times S^{2} ; \mathbb{C}^{2}\right)}^{2}
\end{aligned}
$$

Let $\Psi_{T} \in C^{\infty}\left(\Sigma_{T}^{\varphi} ; \mathbb{C}^{4}\right)$ and $\Psi \in C^{\infty}\left(K_{T}^{\varphi} ; \mathbb{C}^{4}\right)$ be the associated solution of the Dirac equation. Then $\Psi(\varphi(\widehat{r}), \widehat{r}, \omega) \in H^{1}\left(\left[-T_{\varphi}, T_{\varphi}\right] ;\left(H^{\infty}\left(S^{2}\right)\right)^{4}\right)$ and as in Section 7.1 we 
find using Stokes' theorem:

$$
\begin{array}{r}
\int_{-T_{\varphi}}^{T_{\varphi}} \int_{S^{2}}|\Psi|^{2} \mathrm{~d} \widehat{r} \mathrm{~d} \omega \\
=\int_{-T_{\varphi}}^{T_{\varphi}} \int_{S^{2}}\left(\left(1-\varphi^{\prime}\right)\left(\left|\Psi_{2}\right|^{2}+\left|\Psi_{3}\right|^{2}\right)+\left(1+\varphi^{\prime}\right)\left(\left|\Psi_{1}\right|^{2}+\left|\Psi_{4}\right|^{4}\right)\right) \\
(\varphi(\widehat{r}), \widehat{r}, \omega) \mathrm{d} \widehat{r} \mathrm{~d} \omega
\end{array}
$$

We want to estimate $\int_{-T_{\varphi}}^{T_{\varphi}} \int_{S^{2}}|H \Psi|^{2} \mathrm{~d} \widehat{r} \mathrm{~d} \omega$. To this purpose we introduce a sequence of smooth functions $\varphi_{\epsilon}:\left[-T_{\varphi_{\epsilon}}^{1}, T_{\varphi_{\epsilon}}^{2}\right] \rightarrow \mathbb{R}$ such that

$$
\left\{\begin{array}{l}
{\left[-T_{\varphi}, T_{\varphi}\right] \subseteq\left[-T_{\varphi_{\epsilon}}^{1}, T_{\varphi_{\epsilon}}^{2}\right],} \\
\left|\varphi_{\epsilon}^{\prime}(\widehat{r})\right| \leq \tilde{\widetilde{\alpha}}<1, \\
\varphi_{\epsilon} \longrightarrow \varphi L^{\infty}\left(\left[-T_{\varphi}, T_{\varphi}\right]\right), \\
\varphi_{\epsilon}^{\prime} \longrightarrow \varphi^{\prime} \text { a.e. }\left[-T_{\varphi}, T_{\varphi}\right], \\
\varphi_{\epsilon}(\widehat{r}) \leq \varphi(\widehat{r}), \quad \forall \widehat{r} \in\left[-T_{\varphi}, T_{\varphi}\right], \\
\varphi_{\epsilon}\left(-T_{\varphi_{\epsilon}}^{1}\right)=T=\varphi_{\epsilon}\left(T_{\varphi_{\epsilon}}^{2}\right) .
\end{array}\right.
$$

This approximation can be achieved by convolution with smooth functions (see [32, Lemma 3]). Note that we may have to replace the approximation by $\varphi_{\epsilon}-\left|\varphi-\varphi_{\epsilon}\right|_{L^{\infty}}$ to achieve $(7.17)$. In order to compute $(H \Psi)\left(\varphi_{\epsilon}(\widehat{r}), \widehat{r}, \omega\right)$ we introduce the change of variables

$$
\tau=t-\varphi_{\epsilon}(\widehat{r}), x=\widehat{r} \Longrightarrow \partial_{t}=\partial_{\tau}, \partial_{\widehat{r}}=\partial_{x}-\varphi_{\epsilon}^{\prime}(\widehat{r}) \partial_{\tau}
$$

We have

$$
\partial_{t} \Psi=i H \Psi \Longleftrightarrow \partial_{\tau} \Psi=\left(1+\Gamma^{1} \varphi_{\epsilon}^{\prime}\right)^{-1}\left(\Gamma^{1} \partial_{x} \Psi+i\left(P_{\omega}+W\right) \Psi\right) .
$$

Using (7.18) we calculate

$$
\begin{aligned}
H \Psi=\operatorname{Diag} & \left(\frac{1}{1+\varphi_{\epsilon}^{\prime}},-\frac{1}{1-\varphi_{\epsilon}^{\prime}},-\frac{1}{1-\varphi_{\epsilon}^{\prime}}, \frac{1}{1+\varphi_{\epsilon}^{\prime}}\right) D_{x} \Psi \\
+ & \operatorname{Diag}\left(\frac{1}{1+\varphi_{\epsilon}^{\prime}}, \frac{1}{1-\varphi_{\epsilon}^{\prime}}, \frac{1}{1-\varphi_{\epsilon}^{\prime}}, \frac{1}{1+\varphi_{\epsilon}^{\prime}}\right)\left(P_{\omega}+W\right) \Psi
\end{aligned}
$$

Putting $g_{\epsilon}(\widehat{r}, \omega)=\Psi\left(\varphi_{\epsilon}(\widehat{r}), \widehat{r}, \omega\right)$ we find

$$
\begin{aligned}
(H \Psi)\left(\varphi_{\epsilon}(\widehat{r}), \widehat{r}, \omega\right)= & \left(\operatorname{Diag}\left(\frac{1}{1+\varphi_{\epsilon}^{\prime}},-\frac{1}{1-\varphi_{\epsilon}^{\prime}},-\frac{1}{1-\varphi_{\epsilon}^{\prime}}, \frac{1}{1+\varphi_{\epsilon}^{\prime}}\right) D_{\widehat{r}} g_{\epsilon}\right)(\widehat{r}, \omega) \\
& +\left(\operatorname{Diag}\left(\frac{1}{1+\varphi_{\epsilon}^{\prime}}, \frac{1}{1-\varphi_{\epsilon}^{\prime}}, \frac{1}{1-\varphi_{\epsilon}^{\prime}}, \frac{1}{1+\varphi_{\epsilon}^{\prime}}\right)\left(P_{\omega}+W\right) g_{\epsilon}\right)(\widehat{r}, \omega) \\
(7.19) \quad & : g_{\epsilon}^{H} .
\end{aligned}
$$


Using Stokes' theorem we obtain

$$
\begin{aligned}
\int_{-T_{\varphi_{\epsilon}}^{1}}^{T_{\varphi_{\epsilon}}^{2}} \int_{S^{2}}|H \Psi|^{2} \mathrm{~d} \widehat{r} \mathrm{~d} \omega=\|(1 & \left.-\varphi_{\epsilon}^{\prime}\right)^{\frac{1}{2}}\left(g_{\epsilon}^{H}\right)_{2,3} \|_{L^{2}\left(\left[-T_{\varphi_{\epsilon}}^{1}, T_{\varphi_{\epsilon}}^{2}\right] \times S^{2} ; \mathbb{C}^{2}\right)}^{2} \\
& +\left\|\left(1+\varphi_{\epsilon}^{\prime}\right)^{\frac{1}{2}}\left(g_{\epsilon}^{H}\right)_{1,4}\right\|_{L^{2}\left(\left[-T_{\varphi_{\epsilon}}^{1}, T_{\varphi_{\epsilon}}^{2}\right] \times S^{2} ; \mathbb{C}^{2}\right)}^{2} .
\end{aligned}
$$

Using $T_{\varphi_{\epsilon}}^{j} \rightarrow T_{\varphi}, j=1,2$ as well as the fact that $\Psi$ is smooth we can take the limit in (7.20) and find

$$
\begin{aligned}
\int_{-T_{\varphi}}^{T_{\varphi}} \int_{S^{2}}|H \Psi|^{2} \mathrm{~d} \widehat{r} \mathrm{~d} \omega=\|(1 & \left.-\varphi^{\prime}\right)^{\frac{1}{2}}\left(g_{\varphi}^{H}\right)_{2,3} \|_{L^{2}\left(\left[-T_{\varphi}, T_{\varphi}\right] \times S^{2} ; \mathbb{C}^{2}\right)}^{2} \\
& +\left\|\left(1+\varphi^{\prime}\right)^{\frac{1}{2}}\left(g_{\varphi}^{H}\right)_{1,4}\right\|_{L^{2}\left(\left[-T_{\varphi}, T_{\varphi}\right] \times S^{2} ; \mathbb{C}^{2}\right)}^{2}
\end{aligned}
$$

Putting (7.16) and (7.21) together we find

$$
\|\Psi\|_{\mathscr{H}_{T, \varphi}^{1}}^{2}=\|g\|_{\widetilde{H}_{\varphi}^{1}}^{2} .
$$

The equality (7.22) shows that the trace operator

$$
\Psi_{T} \in C^{\infty}\left(\Sigma_{T}^{\varphi} ; \mathbb{C}^{4}\right) \longmapsto \Psi(\varphi(\widehat{r}), \widehat{r}, \omega)
$$

possesses an extension to a bounded operator

$$
\widehat{\mathcal{J}} \in \mathscr{L}\left(\mathcal{H}_{T, \varphi}^{1}, \widetilde{H}_{\varphi}^{1}\right)
$$

The result of this section is:

THEOREM 7.3. - $\widehat{\mathcal{J}}$ is an isometry.

Proof. - Because of (7.22) we only need to prove surjectivity. We have to construct for $g \in \widetilde{H}_{\varphi}^{1}$ a solution of

$$
\partial_{t} \Psi=i H \Psi, \quad \Psi(\varphi(\widehat{r}), \widehat{r}, \omega)=g(\widehat{r}, \omega) .
$$

We first suppose $g \in C^{\infty}\left(\left[-T_{\varphi}, T_{\varphi}\right] \times S^{2} ; \mathbb{C}^{2}\right)$ and consider the approximate problem

$$
\partial_{t} \Psi^{\epsilon}=i H \Psi^{\epsilon}, \quad \Psi^{\epsilon}\left(\varphi_{\epsilon}(\widehat{r}), \widehat{r}, \omega\right)=g(\widehat{r}, \omega),
$$

where $\varphi_{\epsilon}$ is as before and $g(\widehat{r}, \omega)$ is identified with a smooth extension on $\left[-T_{\varphi_{\epsilon}}^{1}, T_{\varphi_{\epsilon}}^{2}\right] \times S^{2}$. As $\varphi_{\epsilon}$ is smooth it is well known that (7.24) possesses a smooth solution and we have the estimate

$$
\left\|\Psi^{\epsilon}\right\|_{\mathcal{H}_{T, \varphi}^{1}}^{2} \leq\left\|\Psi^{\epsilon}\right\|_{\mathcal{H}_{T, \varphi_{\epsilon}}^{1}}^{2}=\|g\|_{\widetilde{H}_{\varphi_{\epsilon}}^{1}}^{2}
$$

Similar estimates hold on $\mathcal{H}_{t, \varphi}^{1}, \min \varphi \leq t \leq T=\max \varphi$. As the R.H.S. of (7.25) is uniformly bounded we find uniform bounds (here we also use $\varphi_{\epsilon} \leq \varphi$ ):

$$
\left\|\Psi^{\epsilon}\right\|_{\left(H^{1}\left(K_{T}^{\varphi}\right)\right)^{4}} \lesssim 1, \quad\left\|\Psi^{\epsilon}\right\|_{\mathcal{H}_{T, \varphi}^{1}} \lesssim 1
$$

We can therefore extract a subsequence which we denote again $\Psi^{\epsilon}$ such that

$$
\Psi^{\epsilon} \rightarrow \Psi \quad \mathcal{H}_{T, \varphi}^{1}, \quad \Psi^{\epsilon} \rightarrow \Psi \quad\left(H^{1}\left(K_{T}^{\varphi}\right)\right)^{4}, \quad \Psi^{\epsilon} \rightarrow \Psi \quad\left(H^{s}\left(K_{T}^{\varphi}\right)\right)^{4},
$$


for $\frac{1}{2}<s<1$. The limit $\Psi$ is a solution of the Dirac equation. We have to check that $\Psi(\varphi(\widehat{r}), \widehat{r}, \omega)=g(\widehat{r}, \omega)$. To this aim we estimate for $\epsilon$ small:

$$
\begin{gathered}
\int_{-T_{\varphi}}^{T_{\varphi}} \int_{S^{2}}\left|\Psi^{\epsilon}(\varphi(\widehat{r}), \widehat{r}, \omega)-g(\widehat{r}, \omega)\right|^{2} \mathrm{~d} \widehat{r} \mathrm{~d} \omega \\
=\int_{-T_{\varphi}}^{T_{\varphi}} \int_{S^{2}}\left|\Psi^{\epsilon}(\varphi(\widehat{r}), \widehat{r}, \omega)-\Psi^{\epsilon}\left(\varphi_{\epsilon}(\widehat{r}), \widehat{r}, \omega\right)\right|^{2} \mathrm{~d} \widehat{r} \mathrm{~d} \omega \\
=\int_{-T_{\varphi}}^{T_{\varphi}} \int_{S^{2}}\left|\int_{\varphi_{\epsilon}(\widehat{r})}^{\varphi(\widehat{r})} \partial_{t} \Psi^{\epsilon}(t, \widehat{r}, \omega)\right|^{2} \mathrm{~d} t \mathrm{~d} \widehat{r} \mathrm{~d} \omega \\
\leq \int_{-T_{\varphi}}^{T_{\varphi}} \int_{S^{2}} \int_{\varphi_{\epsilon}(\widehat{r})}^{\varphi(\widehat{r})}\left|\partial_{t} \Psi^{\epsilon}(t, \widehat{r}, \omega)\right|^{2} \mathrm{~d} t\left|\varphi(\widehat{r})-\varphi_{\epsilon}(\widehat{r})\right| \mathrm{d} \widehat{r} \mathrm{~d} \omega \\
\quad \leq\left|\varphi-\varphi_{\epsilon}\right|_{L^{\infty}} \int_{\frac{1}{2} \min \varphi}^{\max \varphi} \int_{-T_{\varphi}}^{T_{\varphi}} \int_{S^{2}}\left|H \Psi^{\epsilon}(t, \widehat{r}, \omega)\right|^{2} \mathrm{~d} \widehat{r} \mathrm{~d} \omega \mathrm{d} t \\
\quad \lesssim\left|\varphi_{\epsilon}-\varphi\right|_{L^{\infty}} \cdot\left\|H \Psi^{\epsilon}\right\|_{\mathcal{H}_{T, \varphi}}^{2} \lesssim\left|\varphi_{\epsilon}-\varphi\right|_{L^{\infty}} \longrightarrow 0, \quad \epsilon \rightarrow 0 .
\end{gathered}
$$

Here we have used the Cauchy-Schwarz inequality. On the other hand we know that

$$
\left\|\Psi^{\epsilon}(\varphi(\widehat{r}), \widehat{r}, \omega)-\Psi(\varphi(\widehat{r}), \widehat{r}, \omega)\right\|_{L^{2}\left(\left(\left[-T_{\varphi}, T_{\varphi}\right] \times S^{2}, \mathrm{~d} \widehat{r} \mathrm{~d} \omega\right) ; \mathbb{C}^{4}\right)} \leq\left\|\Psi^{\epsilon}-\Psi\right\|_{\left(H^{s}\left(K_{T}^{\varphi}\right)\right)^{4}} \rightarrow 0 .
$$

It follows that $\Psi(\varphi(\widehat{r}), \widehat{r}, \omega)=g(\widehat{r}, \omega)$. The solution $\Psi$ satisfies

$$
\|\Psi\|_{\mathcal{H}_{T, \varphi}^{1}} \leq\|g\|_{\widetilde{H}_{\varphi}^{1}}, \quad\|\Psi\|_{\left(H^{1}\left(K_{T}^{\varphi}\right)\right)^{4}} \lesssim\|g\|_{\widetilde{H}_{\varphi}^{1}} .
$$

If $g \in \widetilde{H}_{\varphi}^{1}$, then we approximate it by a sequence $g^{n}$ of $C^{\infty}$ functions. Then by (7.26) the associated sequence $\Psi^{n}$ of solutions converges to some $\Psi$ in the norms $\mathcal{H}_{T, \varphi}^{1},\left(H^{1}\left(K_{T}^{\varphi}\right)\right)^{4}$. As $\Psi \in\left(H^{1}\left(K_{T}^{\varphi}\right)\right)^{4}$, the trace $\left.\Psi\right|_{\Lambda_{\varphi}}$ exists and we have

$$
\begin{aligned}
\int_{-T_{\varphi}}^{T_{\varphi}} \int_{S^{2}}|\Psi(\varphi(\widehat{r}), \widehat{r}, \omega)-g(\widehat{r}, \omega)|^{2} \mathrm{~d} \widehat{r} \mathrm{~d} \omega \leq\left\|\Psi-\Psi^{n}\right\|_{\left(H^{s}\left(K_{T}^{\varphi}\right)\right)^{4}}^{2} & \\
& +\int_{-T_{\varphi}}^{T_{\varphi}} \int_{S^{2}}\left|g^{n}-g\right|^{2} \mathrm{~d} \widehat{r} \mathrm{~d} \omega \longrightarrow 0
\end{aligned}
$$

and thus $\widehat{\mathcal{J}} \Psi=g$. This concludes the proof of the theorem.

\subsection{Proof of Theorems 7.1 and 7.2}

Because of (7.3) and (7.11) we only have to show surjectivity. Let $\lambda<1$ and $\varphi_{\lambda}=\lambda|\widehat{r}|$. Then the hypersurface $\Lambda_{\varphi_{\lambda}}=\left\{\left(\varphi_{\lambda}(\widehat{r}), \widehat{r}, \omega\right) ;|\widehat{r}| \leq T, \omega \in S^{2}\right\}$ is a lipschitz space-like hypersurface. We first suppose

$$
g_{2,3} \in C^{\infty}\left([-T, 0] \times S^{2} ; \mathbb{C}^{2}\right) \quad \text { and } \quad \widehat{g}_{1,4} \in C^{\infty}\left([0, T] \times S^{2} ; \mathbb{C}^{2}\right)
$$


(which we extend to smooth functions on $[-T / \lambda, 0] \times S^{2} \operatorname{resp} .[0, T / \lambda] \times S^{2}$ ). Let $g_{1,4}(\widehat{r}, \omega)$ and $\widehat{g}_{2,3}(\widehat{r}, \omega)$ be the solutions of (7.4) and (7.5). We consider the approximate problem:

$$
\begin{cases}\partial_{t} \Psi^{\lambda}=i H \Psi^{\lambda}, & \\ \Psi^{\lambda}(-\lambda \widehat{r}, \widehat{r}, \omega)=g(\widehat{r}, \omega), & -T / \lambda \leq \widehat{r} \leq 0, \\ \Psi^{\lambda}(\lambda \widehat{r}, \widehat{r}, \omega)=\widehat{g}(\widehat{r}, \omega), & 0 \leq \widehat{r} \leq T / \lambda .\end{cases}
$$

We put

$$
\widetilde{g}(\widehat{r}, \omega):= \begin{cases}g(\widehat{r}, \omega) & -T / \lambda \leq \widehat{r} \leq 0, \\ \widehat{g}(\widehat{r}, \omega) & 0 \leq \widehat{r} \leq T / \lambda\end{cases}
$$

Starting with $\widetilde{g}$ we define $\widetilde{g}_{\varphi_{\lambda}}^{H}$ as in (7.14). For $-T / \lambda \leq \widehat{r} \leq 0$ we have

$$
\begin{aligned}
\widetilde{g}_{\varphi_{\lambda}}^{H}(\widehat{r}, \omega):=( & \left.\operatorname{Diag}\left(\frac{1}{1-\lambda},-\frac{1}{1+\lambda},-\frac{1}{1+\lambda}, \frac{1}{1-\lambda}\right) D_{\widehat{r}} g\right)(\widehat{r}, \omega) \\
& +\left(\operatorname{Diag}\left(\frac{1}{1-\lambda}, \frac{1}{1+\lambda}, \frac{1}{1+\lambda}, \frac{1}{1-\lambda}\right)\left(P_{\omega}+W\right) g\right)(\widehat{r}, \omega) .
\end{aligned}
$$

Note that the first and fourth components are zero because $g$ is a solution of (7.4). In a similar way we find for $0 \leq \widehat{r} \leq \frac{T}{\lambda}$ :

$$
\begin{aligned}
\widetilde{g}_{\varphi_{\lambda}}^{H}(\widehat{r}, \omega):=( & \left.\operatorname{Diag}\left(\frac{1}{1+\lambda},-\frac{1}{1-\lambda},-\frac{1}{1-\lambda}, \frac{1}{1+\lambda}\right) D_{\widehat{r}} \widehat{g}\right)(\widehat{r}, \omega) \\
& +\left(\operatorname{Diag}\left(\frac{1}{1+\lambda}, \frac{1}{1-\lambda}, \frac{1}{1-\lambda}, \frac{1}{1+\lambda}\right)\left(P_{\omega}+W\right) \widehat{g}\right)(\widehat{r}, \omega) .
\end{aligned}
$$

Here the second and third components are zero because $\widehat{g}$ is a solution of (7.5). As $\left(g_{2,3}, \widehat{g}_{1,4}\right) \in \widetilde{H}^{1}$ we see that $\widetilde{g} \in \widetilde{H}_{\varphi_{\lambda}}^{1}$. Therefore $(7.27)$ possesses by Theorem 7.3 a unique solution $\Psi^{\lambda}$ satisfying the energy estimate

$$
\left\|\Psi^{\lambda}\right\|_{\mathcal{H}_{T}^{1}}^{2} \leq\left\|\Psi^{\lambda}\right\|_{\mathscr{H}_{\varphi_{\lambda}, T}^{1}}^{2}=\|g\|_{\widetilde{\mathcal{H}}_{\varphi_{\lambda}}^{1}}^{2}
$$

As

$$
\left(\widetilde{g}_{\varphi_{\lambda}}^{H}\right)_{1,4}=0, \forall-T / \lambda \leq \widehat{r} \leq 0, \quad\left(\widetilde{g}_{\varphi_{\lambda}}^{H}\right)_{2,3}=0, \forall 0 \leq \widehat{r} \leq T / \lambda,
$$

we see that the R.H.S. of (7.28) is uniformly bounded. Repeating the argument for the spaces $\mathcal{H}_{\varphi_{\lambda}, t}^{1}, \min \varphi_{\lambda} \leq t \leq T=\max \varphi_{\lambda}$ we see that we can extract a subsequence, still denoted $\Psi^{\lambda}$, such that

$$
\Psi^{\lambda} \rightarrow \Psi \quad \mathcal{H}_{T}^{1}, \quad \Psi^{\lambda} \rightarrow \Psi \quad\left(H^{1}\left(K_{T}\right)\right)^{4}, \quad \Psi^{\lambda} \rightarrow \Psi \quad\left(H^{s}\left(K_{T}\right)\right)^{4},
$$

for $\frac{1}{2}<s<1$. $\Psi$ is a solution of the Dirac equation and we have

$$
\|\Psi\|_{\mathcal{H}_{T}^{1}} \leq\|g\|_{\widetilde{H}^{1}}, \quad\|\Psi\|_{\left(H^{1}\left(K_{T}\right)\right)^{4}} \lesssim\|g\|_{\widetilde{H}^{1}} .
$$


We want to check that

$$
\begin{aligned}
\Psi_{2,3}(-\widehat{r}, \widehat{r}, \omega) & =g_{2,3}(\widehat{r}, \omega), \quad \forall-T \leq \widehat{r} \leq 0, \\
\Psi_{1,4}(\widehat{r}, \widehat{r}, \omega) & =\widehat{g}_{1,4}(\widehat{r}, \omega), \quad \forall 0 \leq \widehat{r} \leq T .
\end{aligned}
$$

In fact we can even show

$$
\Psi(|\widehat{r}|, \widehat{r}, \omega)=\widetilde{g}(\widehat{r}, \omega)
$$

As in Section 7.2 we estimate

$$
\begin{gathered}
\int_{-T}^{T} \int_{S^{2}}\left|\widetilde{g}(\widehat{r}, \omega)-\Psi^{\lambda}(|\widehat{r}|, \widehat{r}, \omega)\right|^{2} \mathrm{~d} \widehat{r} \mathrm{~d} \omega \\
=\int_{-T}^{T} \int_{S^{2}}\left|\Psi^{\lambda}(\lambda|\widehat{r}|, \widehat{r}, \omega)-\Psi^{\lambda}(|\widehat{r}|, \widehat{r}, \omega)\right|^{2} \mathrm{~d} \widehat{r} \mathrm{~d} \omega \\
=\int_{-T}^{T} \int_{S^{2}}\left|\int_{|\widehat{r}|}^{\lambda|\widehat{r}|} \partial_{t} \Psi^{\lambda}(t, \widehat{r}, \omega) \mathrm{d} t\right|^{2} \mathrm{~d} \widehat{r} \mathrm{~d} \omega \mathrm{d} t \\
\leq|\lambda-1| \cdot T \int_{-T}^{T} \int_{S^{2}} \int_{\lambda|\widehat{r}|}^{|\widehat{r}|}\left|\partial_{t} \Psi^{\lambda}(t, \widehat{r}, \omega)\right|^{2} \mathrm{~d} \widehat{r} \mathrm{~d} \omega \mathrm{d} t \\
\quad \leq T|\lambda-1| \cdot\left\|H \Psi^{\lambda}\right\|_{\mathscr{H}_{T}}^{2} \lesssim T|\lambda-1| \longrightarrow 0 .
\end{gathered}
$$

On the other hand

$$
\int_{-T}^{T} \int_{S^{2}}\left|\Psi^{\lambda}(|\widehat{r}|, \widehat{r}, \omega)-\Psi(|\widehat{r}|, \widehat{r}, \omega)\right|^{2} \mathrm{~d} \widehat{r} \mathrm{~d} \omega \leq\left\|\Psi^{\lambda}-\Psi\right\|_{\left(H^{s}\left(K_{T}\right)\right)^{4}}^{2} \longrightarrow 0 .
$$

Thus $\Psi(|\widehat{r}|, \widehat{r}, \omega)=\widetilde{g}(\widehat{r}, \omega)$. If $\left(g_{2,3}, \widehat{g}_{1,4}\right) \in \widetilde{H}^{1}$, we approach it by a sequence $\left(g_{2,3}^{n}, \widehat{g}_{1,4}^{n}\right)$ of smooth data and the corresponding solutions converge to a solution $\Psi$. The trace of $\Psi$ on $\Lambda_{T}$ exists and we show as in the proof of Theorem 7.3 that

$$
\Psi_{2,3}(-\widehat{r}, \widehat{r}, \omega)=g_{2,3}(\widehat{r}, \omega), \quad \Psi_{1,4}(\widehat{r}, \omega)=g_{1,4}(\widehat{r}, \omega) .
$$

If $\left(g_{2,3}, \widehat{g}_{1,4}\right) \in L_{T,-}^{2} \oplus L_{T,+}^{2}$ we again approach it by a sequence of smooth data $\left(g_{2,3}^{n}, \widehat{g}_{1,4}^{n}\right)$. The corresponding solutions are in $\mathcal{H}_{T}^{1}$ and converge to some $\Psi$ in $\mathcal{H}_{T}$. By definition of the extension we have $\mathcal{J} \Psi=g$ (see Remark 7.1). This concludes the proofs of Theorem 7.1 and Theorem 7.2.

\subsection{The characteristic Cauchy problem on $\mathcal{M}_{\text {col }}$}

In this section we want to solve a characteristic Cauchy problem outside the collapsing star. The data will be a function $g_{2,3}(t, \omega)$ for which we suppose

$$
\exists t_{g}, \forall t \geq t_{g}, \quad g_{2,3}(t, \omega)=0 .
$$




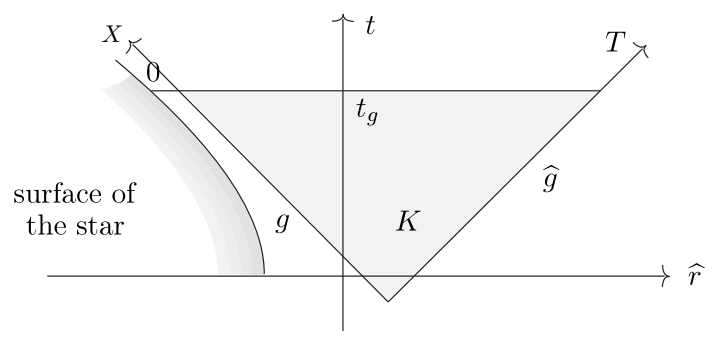

Figure 2. The characteristic Cauchy problem outside the collapsing star

We want to solve the characteristic Cauchy problem

$$
\left\{\begin{array}{l}
\partial_{t} \Psi=i H \Psi, \quad \widehat{z}(t, \theta) \leq \widehat{r} \leq-t+1, t \geq 0 \\
\Psi_{2,3}(t,-t+1, \omega)=g_{2,3}(t, \omega) \\
\sum_{\mu \in\{t, \widehat{r}, \theta, \varphi\}}\left(\mathcal{N}_{\mu} \widehat{\gamma}^{\mu} \Psi\right)(\widehat{z}(t, \theta), \omega)=-i \Psi(\widehat{z}(t, \theta), \omega), \\
t>t_{g}, \widehat{r} \in[\widehat{z}(t, \theta),-t+1] \Longrightarrow \Psi(t, \widehat{r}, \omega)=0
\end{array}\right.
$$

and write the solution as

$$
\Psi(t)=U\left(t, t_{g}\right) \Psi_{K}\left(t_{g}\right),
$$

where $\Psi_{K}\left(t_{g}\right)$ is the solution at time $t_{g}$ of a characteristic problem in $K, K$ as in Figure 2.

We first have to specify the regularity of the data. If $g_{1,4}(t, \omega)=\Psi_{1,4}(t,-t+1, \omega)$, then $g_{1,4}$ is solution of the equation

$$
D_{t} g_{1,4}=\left(\left(P_{\omega}+W\right) g\right)_{1,4}, \quad g_{1,4}\left(t_{g}, \omega\right)=0,
$$

which we can write as

$$
g_{1,4}(t, \omega)=\int_{t_{g}}^{t} V(t, s) S(s) \mathrm{d} s
$$

with a propagator $V(t, s)$ and a source term $S(s)$ associated to (7.32) as in Section 7.1. We then put

$$
g_{2,3}^{H}(t, \omega):=\frac{1}{2}\left(D_{t} g_{2,3}+\left(\left(P_{\omega}+W\right) g\right)_{2,3}\right) .
$$

Let $\hat{H}^{1}\left(\left[0, t_{g}\right] \times S^{2} ; \mathbb{C}^{2}\right)$ be the completion of

$$
\mathscr{C}_{0}=\left\{u \in C^{\infty}\left(\left[0, t_{g}\right] \times S^{2} ; \mathbb{C}^{2}\right) ; u\left(t_{g}, \omega\right)=0, \forall \omega \in S^{2}\right\}
$$

in the norm

$$
\left\|g_{2,3}\right\|_{\hat{H}^{1}}^{2}=2\left(\left\|g_{2,3}\right\|_{L^{2}\left(\left[0, t_{g}\right] \times S^{2} ; \mathbb{C}^{2}\right)}^{2}+\left\|g_{2,3}^{H}\right\|_{L^{2}\left(\left[0, t_{g}\right] \times S^{2} ; \mathbb{C}^{2}\right)}^{2}\right) .
$$

The result of this section is: 
THEOREM 7.4. - Let $g_{2,3} \in \hat{H}^{1}$. Then (7.31) possesses a unique solution $\Psi$ with $\widetilde{\Psi} \in C^{1}(\mathbb{R} ; \mathcal{H}) \cap C\left(\mathbb{R} ; \mathcal{H}^{1}\right)$ and

$$
t \geq 0, \widehat{r} \in[\widehat{z}(t, \theta),-t+1] \Longrightarrow \Psi(t, \widehat{r}, \omega)=\widetilde{\Psi}(t, \widehat{r}, \omega) .
$$

Furthermore, for all $0 \leq t_{0} \leq t_{g}$, we have

$$
\int_{S^{2}} \int_{\widehat{z}\left(t_{0}, \theta\right)}^{-t_{0}+1}|\Psi|^{2}\left(t_{0}, \widehat{r}, \omega\right) \mathrm{d} \widehat{r} \mathrm{~d} \omega=2 \int_{t_{0}}^{t_{g}} \int_{S^{2}}\left|g_{2,3}\right|^{2}(t, \omega) \mathrm{d} t \mathrm{~d} \omega .
$$

Proof. - We first show uniqueness. Let $0 \leq t_{0} \leq t_{g}$,

$$
\begin{aligned}
B_{1} & :=\left\{\left(t_{0}, \widehat{r}, \omega\right) ; \widehat{z}\left(t_{0}, \omega\right) \leq \widehat{r} \leq-t_{0}+1\right\}, \\
B_{2} & :=\left\{\left(t_{g}, \widehat{r}, \omega\right) ; \widehat{z}\left(t_{g}, \theta\right) \leq \widehat{r} \leq-t_{g}+1\right\}, \\
B_{3} & :=\left\{(t, \widehat{z}(t, \theta), \omega) ; t_{0} \leq t \leq t_{g}, \omega \in S^{2}\right\}, \\
B_{4} & :=\left\{(t,-t+1, \omega) ; t_{0} \leq t \leq t_{g}, \omega \in S^{2}\right\}, \\
B & :=B_{1} \cup B_{2} \cup B_{3} \cup B_{4} .
\end{aligned}
$$

By Stokes' theorem we have

$$
\begin{aligned}
& \int_{B} *\left(\phi_{A} \bar{\phi}_{A^{\prime}} \mathrm{d} x^{A A^{\prime}}+\bar{\chi}_{A} \chi_{A^{\prime}} \mathrm{d} x^{A A^{\prime}}\right)=0 \\
& \Longleftrightarrow \int_{S^{2}} \int_{\widehat{z}\left(t_{0}, \theta\right)}^{-t_{0}+1}|\Psi|^{2}\left(t_{0}, \widehat{r}, \omega\right) \mathrm{d} \widehat{r} \mathrm{~d} \omega=2 \int_{t_{0}}^{t_{g}} \int_{S^{2}}\left|g_{2,3}\right|^{2}(t, \omega) \mathrm{d} t \mathrm{~d} \omega .
\end{aligned}
$$

Indeed

$$
\int_{B_{2}} *\left(\phi_{A} \bar{\phi}_{A^{\prime}} \mathrm{d} x^{A A^{\prime}}+\bar{\chi}_{A} \chi_{A^{\prime}} \mathrm{d} x^{A A^{\prime}}\right)=0
$$

because the solution is zero on $B_{2}$ and

$$
\int_{B_{3}} *\left(\phi_{A} \bar{\phi}_{A^{\prime}} \mathrm{d} x^{A A^{\prime}}+\bar{\chi}_{A} \chi_{A^{\prime}} \mathrm{d} x^{A A^{\prime}}\right)=0
$$

because $\mathcal{N}^{A A^{\prime}}\left(\phi_{A} \bar{\phi}_{A^{\prime}}+\bar{\chi}_{A} \chi_{A^{\prime}}\right)=0$. The equality (7.35) gives the uniqueness result.

Let us now prove existence. Let

$$
K:=\left\{(t, \widehat{r}, \omega) ;-1 \leq t \leq t_{g},-t+1 \leq \widehat{r} \leq t+3\right\} .
$$

We put

$$
\widehat{g}_{1,4}(t, \omega)=\widehat{g}_{1,4}(0, \omega)=g_{1,4}(0, \omega)=\int_{t_{g}}^{0} V(0, s) S(s) \mathrm{d} s .
$$

The characteristic Cauchy problem

$$
\left\{\begin{array}{l}
\partial_{t} \Psi=i H \Psi, \quad(t, \widehat{r}, \omega) \in K \\
\Psi_{2,3}(t,-t+1, \omega)=g_{2,3}(t, \omega) \\
\Psi_{1,4}(t, t+3, \omega)=\widehat{g}_{1,4}(t, \omega)
\end{array}\right.
$$


has, by the results of the previous sections, a unique solution $\Psi_{\widehat{g}}$. We now choose a smooth cut-off $\chi \in C^{\infty}(\mathbb{R})$ with

$$
\chi(x)= \begin{cases}1 & x \leq t_{g}+2 \\ 0 & x \geq t_{g}+\frac{5}{2}\end{cases}
$$

and put

$$
\Psi\left(t_{g}, \widehat{r}, \omega\right)= \begin{cases}\chi \Psi_{\widehat{g}}\left(t_{g}, \widehat{r}, \omega\right) & \widehat{r} \geq-t_{g}+1 \\ 0 & \widehat{r} \leq-t_{g}+1 .\end{cases}
$$

This defines $\Psi\left(t_{g}, \widehat{r}, \omega\right)$ for all $\widehat{r} \geq \widehat{z}\left(t_{g}, \theta\right)$. Note that because of the finite propagation speed the solution in the domain we are interested in is independent of $\left.\Psi\left(t_{g}, \widehat{r}, \omega\right)\right|_{\left[t_{g}+2, \infty\right) \times S^{2}}$. Because of (7.36) we have $\Psi_{\widehat{g}}\left(t_{g},-t_{g}+1, \omega\right)=0$. Therefore $\Psi\left(t_{g}, \widehat{r}, \omega\right) \in \mathcal{H}^{1}$. The restriction of

$$
\widetilde{\Psi}(t)=\left[U\left(t, t_{g}\right) \Psi\left(t_{g}\right)\right]_{H}
$$

to $\left\{(t, \widehat{r}, \omega) ; 0 \leq t \leq t_{g}, \widehat{z}(t, \theta) \leq \widehat{r} \leq-t+1, \omega \in S^{2}\right\}$ solves the problem.

REMARK 7.2. - (a) We could of course permit data which do not vanish on $B_{2}$ and proceed as in the preceding sections. However in the next sections, we shall need a description of the solutions as in (7.38).

(b) Let $\Psi \in C^{1}(\mathbb{R} ; \mathcal{H}) \cap C\left(\mathbb{R} ; \mathcal{H}^{1}\right)$ be a solution of the characteristic problem

$$
\left.\begin{array}{l}
\partial_{t} \Psi=i H \Psi \\
\Psi_{2,3}(t,-t+1, \omega)=g_{2,3}(t, \omega) .
\end{array}\right\}
$$

Then we have the following energy estimate:

$$
2 \int_{\mathbb{R}} \int_{S^{2}}\left|g_{2,3}\right|^{2}(s, \omega) \mathrm{d} s \mathrm{~d} \omega \leq\|\Psi(t)\|_{\mathscr{H}}^{2}, \quad \forall t .
$$

Indeed Stokes' theorem gives for $T>0$ :

$$
\begin{gathered}
\int_{1}^{2 T+1} \int_{S^{2}}|\Psi|^{2}(0, \widehat{r}, \omega) \mathrm{d} \widehat{r} \mathrm{~d} \omega \\
=2\left(\int_{-T}^{0} \int_{S^{2}}\left|g_{2,3}\right|^{2}(t, \omega) \mathrm{d} t \mathrm{~d} \omega+\int_{-T}^{0} \int_{S^{2}}\left|\Psi_{1,4}\right|^{2}(t, t+2 T+1, \omega) \mathrm{d} t \mathrm{~d} \omega\right) \\
\Longrightarrow 2 \int_{-\infty}^{0} \int_{S^{2}}\left|g_{2,3}\right|^{2}(t, \omega) \mathrm{d} t \mathrm{~d} \omega \leq \int_{1}^{\infty} \int_{S^{2}}|\Psi|^{2}(0, \widehat{r}, \omega) \mathrm{d} \widehat{r} \mathrm{~d} \omega .
\end{gathered}
$$

In the same way we can show

$$
2 \int_{0}^{\infty} \int_{S^{2}}\left|g_{2,3}\right|^{2}(t, \omega) \mathrm{d} t \mathrm{~d} \omega \leq \int_{-\infty}^{1} \int_{S^{2}}|\Psi|^{2}(0, \widehat{r}, \omega) \mathrm{d} \widehat{r} \mathrm{~d} \omega .
$$

Thanks to inequality (7.40) we can extend the trace operator $T: \Psi_{T} \mapsto \Psi(t,-t+1, \omega)$ to a bounded operator $T \in \mathscr{L}\left(\mathcal{H} ; L^{2}\left(\left(\mathbb{R} \times S^{2} \mathrm{~d} t \mathrm{~d} \omega\right) ; \mathbb{C}^{2}\right)\right)$. 


\section{CHAPTER 8}

\section{REDUCTIONS}

In this chapter we present the basic analytic problem that we have to solve in order to prove the main theorem.

\subsection{The key theorem}

Let $n \in \mathbb{Z}+\frac{1}{2}, \widetilde{\Sigma}=\mathbb{R} \times[0,2 \pi]_{\varphi} \times[0, \pi]_{\theta}$,

$$
\eta^{n}=\frac{q Q r_{+}}{r_{+}^{2}+a^{2}}+\frac{a n}{r_{+}^{2}+a^{2}}, \quad \mu^{n}=\mathrm{e}^{\sigma \eta^{n}}
$$

(see (5.28) and Remark 6.1). Theorem 5.1 will follow from

TheOREM 8.1 (Key theorem). - Let $f(\widehat{r}, \omega)=\mathrm{e}^{i n \varphi} f^{n}(\widehat{r}, \theta) \in\left(C_{0}^{\infty}(\widetilde{\Sigma})\right)^{4}$. Then

$$
\begin{aligned}
\lim _{T \rightarrow \infty}\left\|\mathbf{1}_{[0, \infty)}\left(H_{0}\right) U(0, T) f\right\|_{0}^{2}=\left\|\mathbf{1}_{[0, \infty)}(H) \mathbf{1}_{\mathbb{R}^{-}}\left(P^{-}\right) f\right\|^{2} & \\
& +\left\langle\Omega_{\leftarrow}^{-} f, \mu^{n} \mathrm{e}^{\sigma H_{\leftarrow}}\left(1+\mu^{n} \mathrm{e}^{\sigma H_{\leftarrow}}\right)^{-1} \Omega_{\leftarrow}^{-} f\right\rangle .
\end{aligned}
$$

Proof of Theorem 5.1 (using the result of Theorem 8.1). - Using the axial symmetry of the problem it is clear that it is sufficient to show $(5.27)$ for $\Phi_{j}(t, \widehat{r}, \omega)=$ $\mathrm{e}^{i n \varphi} \widetilde{\Phi}_{j}(t, \widehat{r}, \theta)$ with $\eta$ replaced by $\eta^{n}$ and $\mu$ by $\mu^{n}$. We then use the polarization identity to see that it is sufficient to evaluate for $\Phi(t, \widehat{r}, \omega)=\mathrm{e}^{i n \varphi} \widetilde{\Phi}(t, \widehat{r}, \theta) \in\left(C_{0}^{\infty}\left(\mathcal{M}_{\text {col }}\right)\right)^{4}$ :

$$
\begin{aligned}
\lim _{T \rightarrow \infty} \omega_{\text {col }}\left(\Psi_{\text {col }}^{*}\left(\Phi^{T}\right) \Psi_{\text {col }}\left(\Phi^{T}\right)\right) & =\lim _{T \rightarrow \infty}\left\|\mathbf{1}_{[0, \infty)}\left(H_{0}\right) S_{\text {col }} \Phi^{T}\right\|_{0}^{2} \\
& =\lim _{T \rightarrow \infty}\left\|\mathbf{1}_{[0, \infty)}\left(H_{0}\right) U(0, T) S \Phi\right\|_{0}^{2} .
\end{aligned}
$$

Here we have used that for $T>0$ sufficiently large we have

$$
S_{\mathrm{col}} \Phi^{T}=U(0, T) S \Phi .
$$

By a change of variables and using the compact support of $\Phi$ we have indeed

$$
S_{\mathrm{col}} \Phi^{T}=\int_{a}^{b} U(0, T) U(T, s+T) \Phi(s) \mathrm{d} s, \quad-\infty<a<b<\infty .
$$


There exist $\widehat{r}_{1}, \widehat{r}_{2}$ such that

$$
\forall a \leq s \leq b, \quad \operatorname{supp} \mathrm{e}^{-i s H} \Phi(s, \widehat{r}, \omega) \subset\left[\widehat{r}_{1}, \widehat{r}_{2}\right] \times S^{2}
$$

In order to replace $U(T, s+T)$ by $\mathrm{e}^{-i s H}$ it is sufficient to choose $T$ large enough such that

$$
\widehat{z}(t, \theta)<\widehat{r}_{1}, \quad \forall t \geq \min \{a+T, T\}, \theta \in[0, \pi]
$$

Now using (8.2) and Theorem 8.1 we obtain

$$
\begin{aligned}
& \lim _{T \rightarrow \infty} \omega_{\text {col }}\left(\Psi_{\text {col }}^{*}\left(\Phi^{T}\right) \Psi_{\text {col }}\left(\Phi^{T}\right)\right)=\left\|\mathbf{1}_{[0, \infty)}(H) \mathbf{1}_{\mathbb{R}^{-}}\left(P^{-}\right) S \Phi\right\|^{2} \\
&+\left\langle\Omega_{\leftarrow}^{-} S \Phi, \mu^{n} \mathrm{e}^{\sigma H \leftarrow}\left(1+\mu^{n} \mathrm{e}^{\sigma H}\right)^{-1} \Omega_{\leftarrow}^{-} S \Phi\right\rangle \\
&=\left\|\mathbf{1}_{[0, \infty)}(H) S \mathbf{1}_{\mathbb{R}^{-}}\left(P^{-}\right) \Phi\right\|^{2} \\
&+\left\langle S \mathbf{1}_{\mathbb{R}^{+}}\left(P^{-}\right) \Phi, \mu^{n} \mathrm{e}^{\sigma H}\left(1+\mu^{n} \mathrm{e}^{\sigma H}\right)^{-1} S \mathbf{1}_{\mathbb{R}^{+}}\left(P^{-}\right) \Phi\right\rangle .
\end{aligned}
$$

From now on we will always suppose $f(\widehat{r}, \omega)=\mathrm{e}^{i n \varphi} f^{n}(\widehat{r}, \theta)$. The proof of Theorem 8.1 (resp. Theorem 8.2) below will be the purpose of the rest of this paper. It will be accomplished in Chapter 11.

\subsection{Fixing the angular momentum}

Thanks to the cylindrical symmetry of the Kerr-Newman space-time the angular momentum of the solution is preserved. More precisely let for $n \in \mathbb{Z}+\frac{1}{2}$ :

$$
\begin{gathered}
\mathcal{H}^{n}:=\left\{\mathrm{e}^{i n \varphi} u \in \mathcal{H} ; u \in\left(L^{2}(\mathbb{R} \times[0, \pi] ; \mathrm{d} \widehat{r} \sin \theta \mathrm{d} \theta)\right)^{4}\right\} \\
\mathcal{H}_{*}^{n}:=\left\{\mathrm{e}^{i n \varphi} u \in \mathcal{H}_{*} ; u \in\left(L^{2}\left(\mathbb{R} \times[0, \pi] ; \mathrm{d} r_{*} \sin \theta \mathrm{d} \theta\right)\right)^{4}\right\} .
\end{gathered}
$$

Then all the dynamics which were introduced so far preserve these spaces. Note that

$$
\mathcal{H}=\bigoplus_{n} \mathcal{H}^{n}
$$

We also define

$$
\mathcal{H}^{n 1}:=\mathcal{H}^{1} \cap \mathcal{H}^{n}, \quad \mathcal{H}_{*}^{n 1}:=\mathcal{H}_{*}^{1} \cap \mathcal{H}_{*}^{n}
$$


Let us put

$$
\begin{aligned}
& \not D_{\mathfrak{s}}^{n}:=\Gamma^{1} D_{r_{*}}+a_{0}\left(r_{*}\right) \not D_{S^{2}}+b_{0}\left(r_{*}\right) \Gamma^{4}+c^{n}, \\
& \not D^{n}:=h \not_{\mathfrak{s}}^{n} h+V^{n}, \\
& \not_{H}^{n}:=\Gamma^{1} D_{r_{*}}-\frac{a}{r_{+}^{2}+a^{2}} n-\frac{q Q r_{+}}{r_{+}^{2}+a^{2}}, \\
& \not_{\rightarrow}^{n}:=\Gamma^{1} D_{r_{*}}+m \Gamma^{4}, \\
& c^{n}:=n c_{2}^{\varphi}+c_{1}, \quad V^{n}:=n V_{\varphi}+V_{1}, \quad \eta^{n}:=\frac{a n}{r_{+}^{2}+a^{2}}+\frac{q Q r_{+}}{r_{+}^{2}+a^{2}}, \\
& V_{0}^{n}:=n V_{\varphi}+V_{1}=\left(V_{0 i j}^{n}\right)_{i j}, \\
& W^{n}:=h^{2} c_{1}+h^{2} c_{2}^{\varphi} n+\widehat{V}_{\varphi} n+\widehat{V}_{1} .
\end{aligned}
$$

The operators $\mathbb{D}^{n}$, etc. will be understood as operators acting on $\mathcal{H}_{*}^{n}$ with domain $D\left(\mathbb{D}^{n}\right)=\left\{u \in \mathcal{H}_{*}^{n} ; \mathbb{D}^{n} u \in \mathcal{H}_{*}^{n}\right\}$, etc. They are selfadjoint with these domains (see [15, Corollary 3.1]), the graph norms of $\mathbb{D}^{n}$ and $\mathbb{D}_{\mathfrak{s}}^{n}$ are equivalent. We define the operators $\widehat{\not D}^{n}$ and $H^{n}$ by

$$
\widehat{D}^{n}=\mathcal{u \not D} \mathcal{U}^{*}, \quad H^{n}=V \widehat{D}^{n} V^{*} .
$$

Clearly $\widehat{D D}^{n}$ and $H^{n}$ are selfadjoint with domains

$$
D\left(\widehat{\not D}^{n}\right)=\mathcal{U}\left(\not^{n}\right), \quad D\left(H^{n}\right)=v D\left(\widehat{\not D}^{n}\right)=v \mathcal{U} D\left(\not^{n}\right) .
$$

We will also need the operator

$$
H_{\leftarrow}^{n}:=\Gamma^{1} D_{\widehat{r}}-\eta^{n}
$$

In order to describe the precise asymptotic behavior of all coefficients we introduce the following symbol classes as subsets of $C^{\infty}(\Sigma)$ :

$$
\begin{aligned}
& f \in \boldsymbol{S}^{m, n} \quad \text { iff } \forall \alpha, \beta \in \mathbb{N}, \quad \partial_{r_{*}}^{\alpha} \partial_{\theta}^{\beta} f=\left\{\begin{array}{l}
\Theta\left(\left\langle r_{*}\right\rangle^{m-\alpha}\right), r_{*} \rightarrow+\infty, \\
\theta\left(\mathrm{e}^{n \kappa_{+}\left|r_{*}\right|}\right), r_{*} \rightarrow-\infty,
\end{array}\right. \\
& f \in \boldsymbol{S}^{m} \quad \text { iff } \forall \alpha, \beta \in \mathbb{N}, \quad \partial_{r_{*}}^{\alpha} \partial_{\theta}^{\beta} f=\theta\left(\left\langle r_{*}\right\rangle^{m-\alpha}\right),\left|r_{*}\right| \rightarrow \infty .
\end{aligned}
$$

We shall denote $f^{\prime}$ the derivative of $f$ with respect to $r_{*}$ even for functions which depend also on $\omega$. We recall [15, Proposition 3.1, Lemma 3.2]:

Proposition 8.1. - We have

$$
\begin{gathered}
a_{0} \in \boldsymbol{S}^{-1,-1}, \quad b_{0} \in \boldsymbol{S}^{0,-1}, \quad b_{0}^{\prime} \in \boldsymbol{S}^{-2,-1}, \\
\left(c^{n}\right)^{\prime} \in \boldsymbol{S}^{-2,-2}, \quad h^{2}-1 \in \boldsymbol{S}^{-2,-2}, \quad V_{0 i j}^{n} \in \boldsymbol{S}^{-2,-1} .
\end{gathered}
$$


Furthermore there exist two constants $C_{3}>0$ and $\epsilon>0$ such that

$$
\begin{aligned}
& \left(a_{0}\left(r_{*}\right)-\frac{1}{r_{*}}\right)^{(i)}=\Theta\left(\left\langle r_{*}\right\rangle^{-1-\epsilon-i}\right), \quad r_{*} \rightarrow \infty, i=1,2, \\
& \left(a_{0}\left(r_{*}\right)-C_{3} \mathrm{e}^{\kappa_{+} r_{*}}\right)^{(i)}=\Theta\left(\mathrm{e}^{\left(\kappa_{+}+\epsilon\right) r_{*}}\right), \quad r_{*} \rightarrow-\infty, i=1,2, \\
& b_{0}-m=\theta\left(\left\langle r_{*}\right\rangle^{-1}\right), \quad r_{*} \rightarrow \infty, \\
& c^{n}+\eta^{n}=\theta\left(\mathrm{e}^{2 \kappa_{+} r_{*}}\right), \quad r_{*} \rightarrow-\infty .
\end{aligned}
$$

REMARK 8.1. - (i) Properties (8.10), (8.11) imply the existence of two constants $R_{0}>0$ and $C_{0}>0$ such that

$$
\begin{aligned}
& \forall r_{*} \geq R_{0}, \quad \frac{C_{0}^{-1}}{r_{*}} \leq a_{0}\left(r_{*}\right) \leq \frac{C_{0}}{r_{*}} \\
& \forall r_{*} \leq-R_{0}, \quad C_{0}^{-1} \mathrm{e}^{\kappa_{+} r_{*}} \leq a_{0}\left(r_{*}\right) \leq C_{0} \mathrm{e}^{\kappa_{+} r_{*}} .
\end{aligned}
$$

(ii) From the definition of $\widehat{r}$ it is clear that we obtain equivalent statements for $\hat{h}(\widehat{r}, \theta)=h\left(r_{*}(\widehat{r}, \theta), \theta\right)$, etc. if we define the symbol classes with respect to $\widehat{r}$.

\subsection{The basic problem}

For $\nu \in \mathbb{R}$ we put

$$
\begin{aligned}
\Gamma^{\nu} & :=\left(\begin{array}{cc}
0 & \bar{a}_{\nu} \\
a_{\nu} & 0
\end{array}\right), \quad a_{\nu}=i \mathrm{e}^{i \nu} \mathbf{1}_{2}, \\
\not D^{\nu, n} & :=h \not_{\mathfrak{s}}^{\nu, n} h+V^{n}, \quad \not D_{\mathfrak{s}}^{\nu, n}:=\Gamma^{1} D_{r_{*}}+a_{0} \not D_{S^{2}}+b_{0} \Gamma^{\nu}+c^{n}, \\
V^{n} & :=V_{1}^{\nu}+n V_{\varphi}, V_{1}^{\nu}=\mathbb{V}_{0}+\frac{m \sqrt{\Delta}}{\sigma}\left(\rho-\sqrt{r^{2}+a^{2}}\right) \Gamma^{\nu}-\frac{q Q r}{\sigma^{2}}\left(r^{2}+a^{2}-\sigma\right),
\end{aligned}
$$

i.e. $\not D^{\nu, n}$ is obtained from $\not^{n}$ by replacing $\Gamma^{4}$ by $\Gamma^{\nu}$. We define $\not_{\rightarrow}^{\nu, n}$ in the same way. The operators $H^{\nu, n}$ and $H_{\rightarrow}^{\nu, n}$ are defined by

$$
H^{\nu, n}=\vee \mathcal{U} \not^{\nu, n} \mathcal{U}^{*} V^{*}, \quad H_{\rightarrow}^{\nu, n}=v \mathcal{U} \mathbb{D}_{\rightarrow}^{\nu, n} \mathcal{U}^{*} V^{*} .
$$

We also define

$$
\mathcal{H}_{t}^{n}:=\left\{u=\mathrm{e}^{i n \varphi} v \in \mathcal{H}_{t} ; v \in\left(L^{2}\left(\Sigma_{t}^{\mathrm{col}, \varphi}, \mathrm{d} \widehat{r} \sin \theta \mathrm{d} \theta\right)\right)^{4}\right\}
$$

with $\Sigma_{t}^{\mathrm{col}, \varphi}=\{(\widehat{r}, \theta) \in \mathbb{R} \times[0, \pi] ; \widehat{r} \geq \widehat{z}(t, \theta)\}$.

Let us consider the problem

$$
\left\{\begin{array}{l}
\partial_{t} \Phi=i H_{t}^{\nu, n} \Phi, \quad \widehat{r}>\widehat{z}(t, \theta), \\
\sum_{\mu \in\{t, \widehat{r}, \theta, \varphi\}}\left(\mathcal{N}_{\mu} \widehat{\gamma}^{\mu} \Phi\right)(t, \widehat{z}(t, \theta), \omega)=-i \Phi(t, \widehat{z}(t, \theta), \omega), \\
\Phi(t=s, .)=\Phi_{s}(.) \in D\left(H_{s}^{\nu, n}\right)
\end{array}\right.
$$


with

$$
\begin{gathered}
D\left(H_{s}^{\nu, n}\right)=\left\{u \in \mathcal{H} ; H_{s}^{\nu, n} u \in \mathcal{H}_{s}^{n},\right. \\
\left.\sum_{\mu \in\{t, \widehat{r}, \theta, \varphi\}} \mathcal{N}_{\mu} \widehat{\gamma}^{\mu} u(t, \widehat{z}(t, \theta), \omega)=-i u(t, \widehat{z}(t, \theta), \omega)\right\} .
\end{gathered}
$$

Proposition 8.2. - Let $\Psi_{s} \in D\left(H_{s}^{\nu, n}\right)$. Then there exists a unique solution

$$
[\Psi(.)]_{H}=\left[U^{\nu, n}(., s) \Psi_{s}\right]_{H} \in C^{1}\left(\mathbb{R}_{t} ; \mathcal{H}^{n}\right) \cap C\left(\mathbb{R}_{t} ; \mathcal{H}^{n 1}\right)
$$

of (8.14) such that $\Psi(t) \in D\left(H_{t}^{\nu, n}\right)$ for all $t \in \mathbb{R}$. Furthermore we have $\|\Psi(t)\|=$ $\left\|\Psi_{s}\right\|$ and $U^{\nu, n}(t, s)$ possesses an extension to an isometric and strongly continuous propagator from $\mathcal{H}_{s}^{n}$ to $\mathcal{H}_{t}^{n}$ such that for all $\Phi_{s} \in D\left(H_{s}\right)$ we have

$$
\frac{\mathrm{d}}{\mathrm{d} t} U^{\nu, n}(t, s) \Phi_{s}=i H_{t} U^{\nu, n}(t, s) \Phi_{s}
$$

and if $R>\widehat{z}(s, \theta)$ for all $\theta$ we have

$$
\left(\widehat{r}>R \Rightarrow \Phi_{s}(\widehat{r}, \omega)=0\right) \Longrightarrow\left(\widehat{r}>R+|t-s| \Rightarrow\left(U^{\nu, n}(t, s) \Phi_{s}\right)(\widehat{r}, \omega)=0\right) .
$$

Proposition 8.2 is proven in Appendix A. Let us for the moment just note that Proposition 8.2 implies Proposition 4.5. Indeed if we define

$$
U(t, s)=\oplus_{n} \mathrm{e}^{i \nu / 2 \gamma^{5}} U^{\nu, n}(t, s) \mathrm{e}^{-i \nu / 2 \gamma^{5}},
$$

then $U(t, s)$ has the required properties. Let us now consider $H_{\eta^{n}}^{\nu, n}=H^{\nu, n}+\eta^{n}$, $H_{\eta^{n}, t}^{\nu, n}:=H_{t}^{\nu, n}+\eta^{n}$. Clearly

$$
\mathbf{1}_{[0, \infty)}\left(H_{0}^{\nu, n}\right)=\mathbf{1}_{\left[\eta^{n}, \infty\right)}\left(H_{\eta^{n}, 0}^{\nu, n}\right)
$$

and an equivalent equation for $H_{\eta^{n}}^{\nu, n}$. If $U_{\eta^{n}}^{\nu, n}(t, s)$ is the evolution system associated to $H_{\eta^{n}, t}^{\nu, n}$, then we have the relation

$$
U_{\eta^{n}}^{\nu, n}(t, s)=\mathrm{e}^{i(t-s) \eta^{n}} U^{\nu, n}(t, s) .
$$

Let also

$$
H_{\leftarrow, \eta^{n}}=H_{\leftarrow}^{n}+\eta^{n}, \quad H_{\rightarrow, \eta^{n}}^{\nu, n}=H_{\rightarrow}^{\nu, n}+\eta^{n}, \quad W_{\eta^{n}}^{n}=W^{n}+\eta^{n} .
$$

Thus if $f(\widehat{r}, \omega)=\sum_{n} \mathrm{e}^{i n \varphi} f^{n}(\widehat{r}, \theta)$, then

$$
\left\|\mathbf{1}_{[0, \infty)}\left(H_{0}\right) U(0, T) f\right\|_{\mathscr{H}}^{2}=\sum_{n}\left\|\mathbf{1}_{\left[\eta^{n}, \infty\right)}\left(H_{\eta^{n}, 0}^{\nu, n}\right) U_{\eta^{n}}^{\nu, n}(0, T) \mathrm{e}^{i \nu / 2 \gamma^{5}} f^{n}\right\|_{\mathscr{H}^{n}}^{2}
$$

and therefore the key theorem will follow from the following

ThEOREM 8.2 (Key theorem 2). - Let $f(\widehat{r}, \omega)=\mathrm{e}^{i n \varphi} f^{n}(\widehat{r}, \theta) \in\left(C_{0}^{\infty}(\widetilde{\Sigma})\right)^{4}$. Then

$$
\begin{aligned}
& \lim _{T \rightarrow \infty}\left\|\mathbf{1}_{\left[\eta^{n}, \infty\right)}\left(H_{\eta^{n}, 0}^{\nu, n}\right) U_{\eta^{n}}^{\nu, n}(0, T) f\right\|_{0}^{2} \\
& =\left\|\mathbf{1}_{\left[\eta^{n}, \infty\right)}\left(H_{\eta^{n}}^{\nu, n}\right) \mathbf{1}_{\mathbb{R}^{-}}\left(P_{n}^{\nu-}\right) f\right\|^{2} \\
& \quad+\left\langle\Omega_{\leftarrow}^{-, \nu, n} f, \mathrm{e}^{\sigma H_{\leftarrow, \eta^{n}}^{n}}\left(1+\mathrm{e}^{\sigma H_{\leftarrow, \eta^{n}}^{n}}\right)^{-1} \Omega_{\leftarrow}^{-, \nu, n} f\right\rangle,
\end{aligned}
$$

where $\sigma$ is as in Theorem 5.1 . 
Here $\Omega_{\leftarrow}^{-, \nu, n}$ and $P_{n}^{\nu-}$ denote

$$
\begin{gathered}
\Omega_{\leftarrow}^{-, \nu, n}=s-\lim _{t \rightarrow-\infty} \mathrm{e}^{-i t H_{\leftarrow}^{\nu, n}} \mathrm{e}^{i t H^{\nu, n}} \mathbf{1}_{\mathbb{R}^{+}}\left(P_{n}^{\nu,-}\right), \\
P_{n}^{\nu,-}=s-C_{\infty}-\lim _{t \rightarrow-\infty} \mathrm{e}^{-i t H^{\nu, n}} \frac{\widehat{r}}{t} \mathrm{e}^{i t H^{\nu, n}} .
\end{gathered}
$$

\subsection{The mixed problem for the asymptotic dynamics}

In this section we give an explicit formula for the mixed problem for the asymptotic dynamics near the horizon. We consider the problem

$$
\left\{\begin{array}{l}
\partial_{t} \Phi=i H_{\leftarrow, \eta^{n}, t} \Phi, \quad(\widehat{r}, \omega) \in \Sigma_{t}^{\mathrm{col}}, \\
\Phi_{2}(t, \widehat{z}(t, \theta), \omega)=-\hat{Z}(t, \theta) \Phi_{4}(t, \widehat{z}(t, \theta), \omega), \\
\Phi_{3}(t, \widehat{z}(t, \theta), \omega)=\hat{Z}(t, \theta) \Phi_{1}(t, \widehat{z}(t, \theta), \omega), \\
\Phi(t=s, .)=\Phi_{s}(.),
\end{array}\right.
$$

where $\hat{Z}(t, \theta)=\sqrt{(1+\dot{\widehat{z}}(t, \theta)) /(1-\dot{\widehat{z}}(t, \theta))}$. We note that the boundary condition is the MIT condition with $a_{0}=0$ and put

$$
\widehat{w}_{0}(t, \theta):=\left(1-\dot{\widehat{z}}^{2}\right)^{-\frac{1}{2}} \text {. }
$$

For $0>x_{0}>\widehat{z}(0, \theta)$ we define $\widehat{\tau}\left(x_{0}, \theta\right)$ by

$$
\widehat{z}\left(\widehat{\tau}\left(x_{0}, \theta\right), \theta\right)+\widehat{\tau}\left(x_{0}, \theta\right)=x_{0} .
$$

We obtain

$$
\begin{aligned}
\widehat{\tau}\left(x_{0}, \theta\right) & =-\frac{1}{2 \kappa_{+}} \ln \left(-x_{0}\right)+\frac{1}{2 \kappa_{+}} \ln (\widehat{A}(\theta))+\theta\left(x_{0}\right), \quad x_{0} \rightarrow 0^{-}, \\
1+\dot{\widehat{z}}\left(\widehat{\tau}\left(x_{0}, \theta\right)\right) & =-2 \kappa_{+} x_{0}+\Theta\left(x_{0}^{2}\right), \quad x_{0} \rightarrow 0^{-} .
\end{aligned}
$$

We denote $U_{\leftarrow}(t, s)$ the isometric propagator associated to (8.17).

LEMMA 8.1. - For $t \leq s$, given $f \in \mathcal{H}_{s}, u(t)=U_{\leftarrow}(t, s) f$ is given by

$\triangleright$ if $\widehat{r}>\widehat{z}(t, \theta)$ then

$$
u_{2}(t, \widehat{r}, \omega)=f_{2}(\widehat{r}-t+s, \omega), \quad u_{3}(t, \widehat{r}, \omega)=f_{3}(\widehat{r}-t+s, \omega),
$$

$\triangleright$ if $\widehat{r}>s+\widehat{z}(s, \theta)-t$ then

$$
u_{1}(t, \widehat{r}, \omega)=f_{1}(\widehat{r}+t-s, \omega), \quad u_{4}(t, \widehat{r}, \omega)=f_{4}(\widehat{r}+t-s, \omega),
$$

$\triangleright$ if $\widehat{z}(t, \theta)<\widehat{r}<s+\widehat{z}(s, \theta)-t$ then

$$
\begin{aligned}
& u_{1}(t, \widehat{r}, \omega)=\hat{Z}^{-1}(\widehat{\tau}(\widehat{r}+t, \theta), \theta) f_{3}(\widehat{r}+t+s-2 \widehat{\tau}(\widehat{r}+t, \theta), \omega), \\
& u_{4}(t, \widehat{r}, \omega)=-\hat{Z}^{-1}(\widehat{\tau}(\widehat{r}+t, \theta), \theta) f_{2}(\widehat{r}+t+s-2 \widehat{\tau}(\widehat{r}+t, \theta), \omega) .
\end{aligned}
$$




\subsection{The new hamiltonians}

In the remaining chapters we consider the operators $H_{\eta^{n}}^{\nu, n}$ etc. acting on the Hilbert space $\mathcal{H}^{n}$. It is clear that all the results of the preceding chapters hold also for these operators. We define the angular part $P_{\omega}^{\nu, n}$ of $H_{\eta^{n}}^{\nu, n}$ by

$$
P_{\omega}^{\nu, n}:=H_{\eta^{n}}^{\nu, n}-\Gamma^{1} D_{\widehat{r}}-W_{\eta^{n}}^{n} .
$$

The indices $\nu, n, \eta^{n}$ will be suppressed from now on. In particular we have a new hamiltonian which is slightly different from the hamiltonian considered in Chapters 4-8. 



\section{CHAPTER 9}

\section{COMPARISON OF THE DYNAMICS}

Let $\mathcal{J} \in C_{b}^{\infty}(\mathbb{R}), 0<a<b<1$ and

$$
\mathcal{J}(\widehat{r})= \begin{cases}1 & \widehat{r} \leq a, \\ 0 & \widehat{r}>b .\end{cases}
$$

The aim of this chapter is to prove the following

$$
\begin{gathered}
\text { Proposition 9.1. - Let } f(\widehat{r}, \omega)=\mathrm{e}^{i n \varphi} f^{n}(\widehat{r}, \theta) \in\left(C_{0}^{\infty}(\widetilde{\Sigma})\right)^{4}, n \in \mathbb{Z}+\frac{1}{2} \text {. Then } \\
\forall \epsilon>0, \exists t_{0}>0, \forall t_{\epsilon} \geq t_{0}, \exists T_{0}>0, \forall T \geq T_{0}, \\
\left\|\mathcal{J}\left(\widehat{r}+t_{\epsilon}\right)\left(U\left(t_{\epsilon}, T\right) f-U_{\leftarrow}\left(t_{\epsilon}, T\right) \Omega_{\leftarrow}^{-}\right) f\right\|<\epsilon .
\end{gathered}
$$

Proposition 9.1 compares the dynamics $U\left(t_{\epsilon}, T\right)$ and $U_{\leftarrow}\left(t_{\epsilon}, T\right) \Omega_{\leftarrow}$ (see Figure 1 ). The function $\mathcal{J}\left(\widehat{r}+t_{\epsilon}\right)$ is a cut-off in the region we are interested in. The proposition states that in this region the above dynamics are close to each other when $t_{\epsilon}$ becomes large and this uniformly in $T$. To prove Proposition 9.1 we understand both $U\left(t_{\epsilon}, T\right) f$ and $U_{\leftarrow}\left(t_{\epsilon}, T\right) \Omega_{\leftarrow}^{-} f$ as solutions of a characteristic problem. In Section 9.1 we compare the characteristic data, in Section 9.2 we compare the solutions of the characteristic problems for the operators $H$ and $H_{\leftarrow}$. Proposition 9.1 is proven in Section 9.3. We suppose for the whole chapter that $f(\widehat{r}, \omega)=\mathrm{e}^{i n \varphi} f^{n}(\widehat{r}, \theta) \in\left(C_{0}^{\infty}(\widetilde{\Sigma})\right)^{4}$, $\operatorname{supp} f \subset\left[R_{1}, R_{2}\right] \times[0,2 \pi] \times[0, \pi]$.

\subsection{Comparison of the characteristic data}

$$
\begin{aligned}
& \text { Let } \\
& g^{T}(t, \omega):=\left(P_{2,3} U(t, T) f\right)(-t+1, \omega), \quad g_{\leftarrow}^{T}(t, \omega):=\left(P_{2,3} U_{\leftarrow}(t, T) \Omega_{\leftarrow}^{-} f\right)(-t+1, \omega) .
\end{aligned}
$$

Note that it is a priori not clear that $U_{\leftarrow}(t, T) \Omega_{\leftarrow}^{-} f$ is regular enough to take the trace

$$
\left(U_{\leftarrow}(t, T) \Omega_{\leftarrow}^{-} f\right)(-t+1, \omega)
$$

in the usual sense, but it can be taken in the sense of Remark 7.2 (b). 


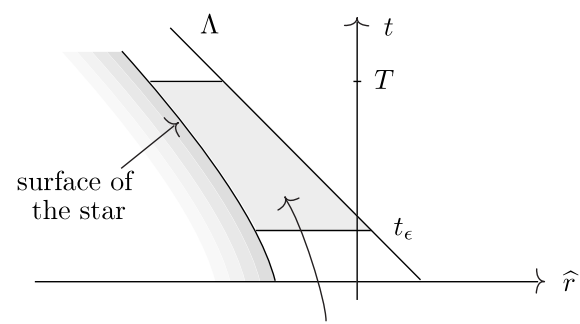

zone where the dynamics are compared

Figure 1. Comparison of the dynamics

LEMMA 9.1. - We have

$$
\int_{0}^{\infty} \int_{S^{2}}\left|g^{T}(t, \omega)-g_{\leftarrow}^{T}(t, \omega)\right|^{2} \mathrm{~d} t \mathrm{~d} \omega \longrightarrow 0, \quad T \rightarrow \infty .
$$

Proof. - First observe that

$$
\begin{aligned}
& g^{T}(t, \omega)=P_{2,3}\left(\mathrm{e}^{i(t-T) H} f\right)(-t+1, \omega), \\
& g_{\leftarrow}^{T}(t, \omega)=P_{2,3}\left(\mathrm{e}^{\left.i(t-T) H \leftarrow \Omega_{\leftarrow}^{-} f\right)(-t+1, \omega) .}\right.
\end{aligned}
$$

Using Lemma 6.6 we see that

$$
\operatorname{supp} f, \operatorname{supp} \Omega_{\leftarrow}^{-} f \subset\left[R_{1}, \infty\right) \times[0,2 \pi] \times[0, \pi] .
$$

By the finite propagation speed this entails

$$
\operatorname{supp}\left(\mathrm{e}^{i(t-T) H} f\right), \operatorname{supp}\left(\mathrm{e}^{\left.i(t-T) H \leftarrow \Omega_{\leftarrow}^{-} f\right) \subset\left[R_{1}-|T-t|, \infty\right) \times[0,2 \pi] \times[0, \pi] .}\right.
$$

If $t>T$, the condition $-t+1 \geq R_{1}-|T-t|$ implies $1 \geq R_{1}+T$ and if $t<T$ the same condition implies $t \leq \frac{1}{2}\left(1+T-R_{1}\right)$. Let $m(T)$ satisfy the conditions of Lemma 6.3. For $T$ sufficiently large we have

$$
\begin{aligned}
I:= & \int_{0}^{\infty} \int_{S^{2}}\left|g^{T}(t, \omega)-g_{\leftarrow}^{T}(t, \omega)\right|^{2} \mathrm{~d} t \mathrm{~d} \omega \\
& \lesssim \int_{0}^{\frac{1}{2}\left(1+T-R_{1}\right)} \int_{S^{2}}\left|P_{2,3} \mathrm{e}^{i t H} \mathbf{1}_{(-1, \infty)}\left(\frac{\widehat{r}}{T-m(T)}\right) \mathrm{e}^{-i T H} f\right|^{2}(-t+1, \omega) \mathrm{d} t \mathrm{~d} \omega \\
& +\int_{0}^{\frac{1}{2}\left(1+T-R_{1}\right)} \int_{S^{2}} \mid P_{2,3} \mathrm{e}^{i t H_{\leftarrow}} \mathbf{1}_{(-1, \infty)}\left(\frac{\widehat{r}}{T-m(T)}\right) \mathrm{e}^{-\left.i T H \leftarrow \Omega_{\leftarrow}^{-} f\right|^{2}(-t+1, \omega) \mathrm{d} t \mathrm{~d} \omega} \\
& +\int_{0}^{\frac{1}{2}\left(1+T-R_{1}\right)} \int_{S^{2}} \mid P_{2,3}\left(\mathrm{e}^{i t H} \mathbf{1}_{(-\infty,-1)}\left(\frac{\widehat{r}}{T-m(T)}\right) \mathrm{e}^{-i T H}\right. \\
& -\mathrm{e}^{i t H_{\leftarrow}} \mathbf{1}_{(-\infty,-1)}\left(\frac{\widehat{r}}{T-m(T)}\right) \mathrm{e}^{\left.-i T H \leftarrow \Omega_{\leftarrow}^{-}\right)\left.f\right|^{2}(-t+1, \omega) \mathrm{d} t \mathrm{~d} \omega} \\
=: & I_{1}+I_{2}+I_{3} .
\end{aligned}
$$


We want to show that

$$
\lim _{T \rightarrow \infty} I_{1}=0
$$

By the energy estimate (7.40) we see that we can replace $f$ by $\chi(H) f, \chi \in C_{0}^{\infty}(\mathbb{R})$, $\operatorname{supp} \chi \subset \mathbb{R} \backslash\left\{-m+\eta^{n}, m+\eta^{n}\right\}$. Let $0<\epsilon_{\chi}<1$ be as in Lemma 6.2. Then

$$
\begin{aligned}
I_{1} & \lesssim \int_{0}^{\frac{1}{2}\left(1+T-R_{1}\right)} \int_{S^{2}}\left|P_{2,3} \mathrm{e}^{i t H} \mathbf{1}_{\left(-1, \frac{1}{2} \epsilon_{\chi}\right)}\left(\frac{\widehat{r}}{T-m(T)}\right) \mathrm{e}^{-i T H} \chi(H) f\right|^{2}(-t+1, \omega) \mathrm{d} t \mathrm{~d} \omega \\
& +\int_{0}^{\frac{1}{2}\left(1+T-R_{1}\right)} \int_{S^{2}}\left|P_{2,3} \mathrm{e}^{i t H_{\leftarrow}} \mathbf{1}_{\left(\frac{1}{2} \epsilon_{\chi}, \infty\right)}\left(\frac{\widehat{r}}{T-m(T)}\right) \mathrm{e}^{-i T H} \chi(H) f\right|^{2}(-t+1, \omega) \mathrm{d} t \mathrm{~d} \omega \\
& =: I_{11}+I_{12}
\end{aligned}
$$

Let us first estimate $I_{12}$. We have

$$
\begin{aligned}
& \operatorname{supp}\left(\mathrm{e}^{i t H} \mathbf{1}_{\left(\frac{1}{2} \epsilon_{\chi}, \infty\right)}\left(\frac{\widehat{r}}{T-m(T)}\right) \mathrm{e}^{-i T H} \chi(H) f\right) \\
& \subset\left(\frac{1}{2} \epsilon_{\chi}(T-m(T))-t, \infty\right) \times[0,2 \pi] \times[0, \pi] .
\end{aligned}
$$

But $-t+1 \geq \frac{1}{2} \epsilon_{\chi}(T-m(T))-t$ implies $1 \geq \frac{1}{2} \epsilon_{\chi}(T-m(T))$. Thus $I_{12}=0$ for $T$ sufficiently large. We now estimate $I_{11}$. By the energy estimate (7.40) we have

$$
\begin{aligned}
I_{11} \leq & \left\|\mathbf{1}_{\left(-1, \frac{1}{2} \epsilon_{\chi}\right)}\left(\frac{\widehat{r}}{T-m(T)}\right) \mathrm{e}^{-i T H} \mathbf{1}_{\mathbb{R}^{+}}\left(P^{-}\right) \chi(H) f\right\| \\
& +\left\|\mathbf{1}_{\left(-1, \frac{1}{2} \epsilon_{\chi}\right)}\left(\frac{\widehat{r}}{T-m(T)}\right) \mathrm{e}^{-i T H} \mathbf{1}_{\mathbb{R}^{-}}\left(P^{-}\right) \chi(H) f\right\| \\
& =I_{11}^{a}+I_{11}^{b} .
\end{aligned}
$$

We have

$$
\lim _{T \rightarrow \infty} I_{11}^{b}=\lim _{T \rightarrow \infty}\left\|\mathbf{1}_{\left(-1, \frac{1}{2} \epsilon_{\chi}\right)}\left(\frac{\widehat{r}}{T-m(T)}\right) \mathbf{1}_{\left(\frac{3}{4} \epsilon_{\chi}, \infty\right)}\left(\frac{\widehat{r}}{T}\right) \mathrm{e}^{-i T H} \chi(H) f\right\|^{2}=0 .
$$

By Lemma 6.3 we have $\lim _{T \rightarrow \infty} I_{11}^{a}=0$. (9.4) follows. In the same manner we can show

$$
\lim _{T \rightarrow \infty} I_{2}=0 .
$$

Let us now estimate $I_{3}$. We have $(t \geq 0)$

$$
\begin{aligned}
\operatorname{supp}\left(\mathrm{e}^{i t H \leftarrow} \mathbf{1}_{(-\infty,-1)}\left(\frac{\widehat{r}}{T-m(T)}\right) \mathrm{e}^{-i T H \leftarrow \Omega_{\leftarrow}^{-} f}\right), \\
\operatorname{supp}\left(\mathrm{e}^{i t H} \mathbf{1}_{(-\infty,-1)}\left(\frac{\widehat{r}}{T-m(T)}\right) \mathrm{e}^{-i T H} f\right) \\
\subset(-\infty, m(T)-T+t) \times[0,2 \pi] \times[0, \pi] .
\end{aligned}
$$


The condition $-t+1 \leq m(T)-T+t$ implies $t \geq \frac{1}{2}(T-m(T)+1)$. In particular $1-\mathcal{J}(-t+1)=0$ for $T$ sufficiently large. Therefore we obtain

$$
\begin{aligned}
I_{3} \leq & \int_{\frac{1}{2}(T-m(T)+1)}^{\frac{1}{2}\left(1+T-R_{1}\right)} \int_{S^{2}} \mid P_{2,3}\left(\mathcal{J}(\widehat{r}) \mathrm{e}^{i t H} \mathbf{1}_{(-\infty,-1)}\left(\frac{\widehat{r}}{T-m(T)}\right) \mathrm{e}^{-i T H}\right. \\
& \left.-\mathrm{e}^{i t H_{\leftarrow}} \mathbf{1}_{(-\infty,-1)}\left(\frac{\widehat{r}}{T-m(T)}\right) \mathrm{e}^{-i T H \leftarrow \Omega_{\leftarrow}^{-}}\right)\left.f\right|^{2}(-t+1, \omega) \mathrm{d} t \mathrm{~d} \omega \\
\lesssim & \int_{\frac{1}{2}(T-m(T)+1)}^{\frac{1}{2}\left(1+T-R_{1}\right)} \int_{S^{2}}\left|P_{2,3} \mathrm{e}^{i t H} \mathbf{1}_{(-1, \infty)}\left(\frac{\widehat{r}}{T-m(T)}\right) \mathrm{e}^{-i T H} f\right|^{2}(-t+1, \omega) \mathrm{d} t \mathrm{~d} \omega \\
& +\int_{\frac{1}{2}(T-m(T)+1)}^{\frac{1}{2}\left(1+T-R_{1}\right)} \int_{S^{2}} \mid P_{2,3} \mathrm{e}^{i t H_{\leftarrow}} \mathbf{1}_{(-1, \infty)}\left(\frac{\widehat{r}}{T-m(T)}\right) \mathrm{e}^{-\left.i T H \leftarrow \Omega_{\leftarrow}^{-} f\right|^{2}} \\
& +\int_{\frac{1}{2}(T-m(T)+1)}^{\frac{1}{2}\left(1+T-R_{1}\right)} \int_{S^{2}} \mid P_{2,3}\left(\mathcal{J}(\widehat{r}) \mathrm{e}^{i(t-T) H} f-\mathrm{e}^{\left.i(t-T) H_{\leftarrow} \Omega_{\leftarrow}^{-}\right)\left.f\right|^{2}(-t+1, \omega) \mathrm{d} t \mathrm{~d} \omega} \mathrm{d} t \mathrm{~d} \omega\right. \\
= & L_{1}+L_{2}+L_{3} .
\end{aligned}
$$

We have for $j=1,2$

$$
0 \leq L_{j} \leq I_{j} \longrightarrow 0, \quad T \rightarrow \infty
$$

We estimate:

$$
\begin{aligned}
L_{3} & \leq \frac{1}{2}\left(m(T)-R_{1}\right) \sup _{\sigma \leq \frac{1}{2}\left(1-\left(T+R_{1}\right)\right)}\left\|\partial(\widehat{r}) \mathrm{e}^{i \sigma H} f-\mathrm{e}^{i \sigma H \leftarrow} \Omega_{\leftarrow}^{-} f\right\|_{L^{\infty}\left(\mathbb{R} ;\left(L^{2}\left(S^{2}\right)\right)^{4}\right)}^{2} \\
& \lesssim \frac{1}{2}\left(m(T)-R_{1}\right) \sup _{\sigma \leq \frac{1}{2}\left(1-\left(T+R_{1}\right)\right)} \| \mathcal{\gamma}(\widehat{r}) \mathrm{e}^{i \sigma H} f-\mathrm{e}^{i \sigma H \leftarrow \Omega_{\leftarrow}^{-} f \|_{H^{1}\left(\mathbb{R} ;\left(L^{2}\left(S^{2}\right)\right)^{4}\right)^{4}}^{2}}
\end{aligned}
$$

We can choose

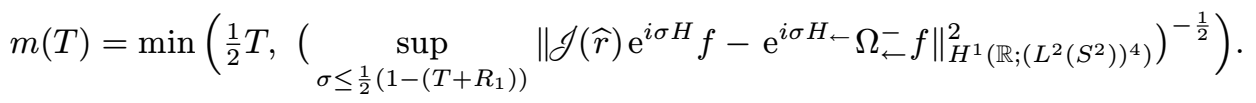

Then by Lemma 6.7 the R.H.S. of (9.5) goes to zero when $T \rightarrow \infty$.

\subsection{Comparison with the asymptotic dynamics}

In the region $\widehat{z}\left(t_{\epsilon}, \theta\right) \leq \widehat{r} \leq a-t_{\epsilon}$ we understand $\mathcal{J}\left(\widehat{r}+t_{\epsilon}\right) U\left(t_{\epsilon}, T\right) f$ as the solution of the characteristic problem on $\mathcal{M}_{\text {col }}$ for the operator $H$ with data $g^{T}$ and

$$
\mathcal{J}\left(\widehat{r}+t_{\epsilon}\right) U_{\leftarrow}\left(t_{\epsilon}, T\right) \Omega_{\leftarrow}^{-} f
$$

as solution of the characteristic problem for the operator $H_{\leftarrow}$ with data $g_{\leftarrow}^{T}$. We would like to solve the characteristic problem for $H$ with data $g_{\leftarrow}^{T}$ and write the solution as

$$
G_{t_{\epsilon}}\left(g_{\leftarrow}^{T}\right)=U\left(t, \frac{1}{2} T+c_{0}\right) \phi\left(\frac{1}{2} T+c_{0}\right),
$$


where $\phi$ is constructed as in (7.38) with $t_{g}=\frac{1}{2} T+c_{0}$ for some $c_{0}>0$. Unfortunately $g_{\leftarrow}^{T}$ will in general not be regular enough to assure that $G_{t_{\epsilon}}\left(g_{\leftarrow}^{T}\right)$ is a strong solution. We shall therefore regularize $\Omega_{\leftarrow}^{-} f$. Let $\chi_{R} \in C_{0}^{\infty}(\mathbb{R})$ with $\left(R \gg R_{1}\right)$

$$
\chi_{R}= \begin{cases}1 & R-1 \geq \widehat{r} \geq R_{1}, \\ 0 & \widehat{r} \leq R_{1}-1, \widehat{r} \geq R .\end{cases}
$$

Let

$$
I_{n}=\left\{\ell ; \ell-\frac{1}{2} \in \mathbb{N}, \ell-|n| \in \mathbb{N}\right\}, \quad \mathscr{I}_{n}^{N}=\left\{\ell \in I_{n} ;|(\ell, n)| \leq N\right\}
$$

and

$$
\Omega_{\leftarrow}^{-} f=\sum_{\ell \in \mathcal{J}_{n}}\left(\Omega_{\leftarrow}^{-} f\right)^{n \ell}, \quad\left(\Omega_{\leftarrow}^{-} f\right)^{n \ell} \in \mathcal{H}^{n \ell}=L^{2}\left((\mathbb{R}, \mathrm{d} \widehat{r}) ; \mathbb{C}^{4}\right) \otimes_{4} Y_{n \ell}, \forall \ell .
$$

We put

$$
\left(\Omega_{\leftarrow}^{-} f\right)^{N}=\sum_{\ell \in \mathcal{J}_{n}^{N}}\left(\Omega_{\leftarrow}^{-} f\right)^{n \ell}, \quad\left(\Omega_{\leftarrow}^{-} f\right)_{R}^{N}=\chi_{R}\left(\Omega_{\leftarrow}^{-} f\right)^{N}
$$

Let

$$
\widetilde{f}:=\left(\Omega_{\leftarrow}^{-} f\right)_{R}^{N}
$$

Clearly we have

$$
\forall \epsilon>0, \exists N_{\epsilon}>0, R_{\epsilon}>0, \forall R \geq R_{\epsilon}, N \geq N_{\epsilon}, \quad\left\|\Omega_{\leftarrow}^{-} f-\left(\Omega_{\leftarrow}^{-} f\right)_{R}^{N}\right\|<\epsilon .
$$

We put

$$
g_{\leftarrow, R}^{T, N}=\left(P_{23} U_{\leftarrow}(t, T)\left(\Omega_{\leftarrow}^{-} f\right)_{R}^{N}\right)(t,-t+1, \omega) .
$$

The functions $\left(g_{\leftarrow, R}^{T, N}\right)_{23}$ are compactly supported. The necessary regularity of $g_{\leftarrow, R}^{T, N}$

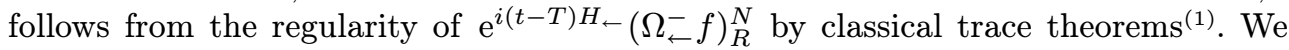
put $c_{0}:=\frac{1}{2}\left(1-R_{0}\right)$ and $R_{0}=R_{1}-1$. Let $\Phi^{R, N}\left(\frac{1}{2} T+c_{0}\right)$ be the solution of the characteristic problem in the whole exterior Kerr-Newman space-time with data $g_{\leftarrow, R}^{T, N}$ on $\{(t, \widehat{r}, \omega) ; 0 \leq t, \widehat{r}=-t+1\}$ as constructed in (7.38) and

$$
\Phi_{\leftarrow}^{R, N}\left(\frac{1}{2} T+c_{0}\right)=\mathrm{e}^{-i\left(\frac{1}{2} T-c_{0}\right) H_{\leftarrow}}\left(\Omega_{\leftarrow}^{-} f\right)_{R}^{N} .
$$

LEMMA 9.2. - We have uniformly in $t_{\epsilon} \geq 0$ :

$$
\left\|\mathcal{J}\left(\widehat{r}+t_{\epsilon}\right) U\left(t_{\epsilon}, \frac{1}{2} T+c_{0}\right)\left(\Phi^{R, N}\left(\frac{1}{2} T+c_{0}, .\right)-\Phi_{\leftarrow}^{R, N}\left(\frac{1}{2} T+c_{0}, .\right)\right)\right\|_{\mathscr{H}_{t_{\epsilon}}} \longrightarrow 0, T \rightarrow \infty .
$$

Proof. - Let

$$
I:=\left\|\mathcal{J}\left(\widehat{r}+t_{\epsilon}\right) U\left(t_{\epsilon}, \frac{1}{2} T+c_{0}\right)\left(\Phi^{R, N}\left(\frac{1}{2} T+c_{0}, .\right)-\Phi_{\leftarrow}^{R, N}\left(\frac{1}{2} T+c_{0}, .\right)\right)\right\|_{\mathcal{H}_{t_{\epsilon}}}^{2} .
$$

First observe that by (7.34) we have

$$
I \leq 2 \int_{t_{\epsilon}}^{\frac{1}{2} T+c_{0}} \int_{S^{2}}\left|\widetilde{g}_{R}^{T, N}-g_{\leftarrow, R}^{T, N}\right|^{2} \mathrm{~d} t \mathrm{~d} \omega
$$

$\overline{(1) \text { Recall that }}\left(U_{\leftarrow}(t, T)\left(\Omega_{\leftarrow}^{-} f\right)_{R}^{N}\right)(-t+1, \omega)=\left(\mathrm{e}^{i(t-T) H_{\leftarrow}}\left(\Omega_{\leftarrow}^{-} f\right)_{R}^{N}\right)(-t+1, \omega)$. 
with

$$
\widetilde{g}_{R}^{T, N}=\left(P_{2,3} \mathrm{e}^{i\left(t-\left(\frac{1}{2} T+c_{0}\right)\right) H} \mathrm{e}^{-i\left(\frac{1}{2} T-c_{0}\right) H \leftarrow \tilde{f}}\right)(-t+1, \omega) .
$$

We proceed as in the proof of Lemma 9.1. Let $m(T)$ satisfy the conditions of Lemma 6.3. Then we have

$$
\begin{aligned}
& I \leq \int_{t_{\epsilon}}^{\frac{1}{2} T+c_{0}} \int_{S^{2}}\left|P_{2,3} \mathrm{e}^{i t H} \mathbf{1}_{(-1, \infty)}\left(\frac{\widehat{r}}{T-m(T)}\right) \mathrm{e}^{-i\left(\frac{1}{2} T+c_{0}\right) H} \mathrm{e}^{-i\left(\frac{1}{2} T-c_{0}\right) H \leftarrow \widetilde{f}}\right|^{2} \\
& \quad+\int_{t_{\epsilon}}^{\frac{1}{2} T+c_{0}} \int_{S^{2}}\left|P_{2,3} \mathrm{e}^{i t H_{\leftarrow}} \mathbf{1}_{(-1, \infty)}\left(\frac{\widehat{r}}{T-m(T)}\right) \mathrm{e}^{-i T H \leftarrow \tilde{f}}\right|^{2}(-t+1, \omega) \mathrm{d} t \mathrm{~d} \omega \\
& \\
& \quad+\int_{t_{\epsilon}}^{\frac{1}{2} T+c_{0}} \int_{S^{2}} \mid P_{2,3}\left(\mathrm{e}^{i t H} \mathbf{1}_{(-\infty,-1)}\left(\frac{\widehat{r}}{T-m(T)}\right) \mathrm{e}^{-i\left(\frac{1}{2} T+c_{0}\right) H} \mathrm{e}^{-i\left(\frac{1}{2} T-c_{0}\right) H_{\leftarrow}}\right. \\
& \quad-\mathrm{e}^{\left.i t H_{\leftarrow} \mathbf{1}_{(-\infty,-1)}\left(\frac{\widehat{r}}{T-m(T)}\right) \mathrm{e}^{-i T H \leftarrow}\right)\left.\tilde{f}\right|^{2}(-t+1, \omega) \mathrm{d} t \mathrm{~d} \omega} \\
& =: I_{1}+I_{2}+I_{3} .
\end{aligned}
$$

Let us first estimate $I_{1}$. We have

$$
\begin{aligned}
& I_{1} \leq \int_{t_{\epsilon}}^{\frac{1}{2} T+c_{0}} \int_{S^{2}}\left|P_{2,3} \mathrm{e}^{i t H} \mathbf{1}_{(-1,1)}\left(\frac{\widehat{r}}{T-m(T)}\right) \mathrm{e}^{-i\left(\frac{1}{2} T+c_{0}\right) H} \mathrm{e}^{-i\left(\frac{1}{2} T-c_{0}\right) H \leftarrow \tilde{f}}\right|^{2} \\
& \quad+\int_{t_{\epsilon}}^{\frac{1}{2} T+c_{0}} \int_{S^{2}}\left|P_{2,3} \mathrm{e}^{i t H} \mathbf{1}_{(1, \infty)}\left(\frac{\widehat{r}}{T-m(T)}\right) \mathrm{e}^{-i\left(\frac{1}{2} T+c_{0}\right) H} \mathrm{e}^{-i\left(\frac{1}{2} T-c_{0}\right) H \leftarrow \tilde{f}}\right|^{2} \\
& (-t+1, \omega) \mathrm{d} t \mathrm{~d} \omega
\end{aligned}
$$

Using a finite propagation speed argument we see that $I_{12}=0$ for $T$ sufficiently large.

We estimate

$$
\begin{aligned}
& I_{11} \leq \| \mathbf{1}_{(-1,1)}(\left.\frac{\widehat{r}}{T-m(T)}\right) \mathrm{e}^{-i\left(\frac{1}{2} T+c_{0}\right) H} \mathrm{e}^{-i\left(\frac{1}{2} T-c_{0}\right) H \leftarrow} \tilde{f} \|^{2} \\
& \leq\left\|\mathbf{1}_{(-1,1)}\left(\frac{\widehat{r}}{T-m(T)}\right) \mathrm{e}^{-i T H} \mathbf{1}_{\mathbb{R}^{+}}\left(P^{-}\right) W_{\leftarrow}^{-} \tilde{f}\right\|^{2} \\
& \quad+\left\|\left(\mathrm{e}^{i\left(\frac{1}{2} T-c_{0}\right) H} \mathrm{e}^{-i\left(\frac{1}{2} T+c_{0}\right) H_{\leftarrow}} P_{\mathcal{H}^{+}}-W_{\leftarrow}^{-}\right) \tilde{f}\right\|^{2} \longrightarrow 0, \quad T \rightarrow \infty,
\end{aligned}
$$

where we have used Lemma 6.3. In the same way we can show

$$
\lim _{T \rightarrow \infty} I_{2}=0
$$


By the same arguments as in the proof of Lemma 9.1 we see that

$$
\begin{array}{r}
I_{3} \leq \int_{\frac{1}{2}(T-m(T)+1)}^{\frac{1}{2} T+c_{0}} \int_{S^{2}}\left|\left(\mathrm{e}^{i\left(t-\left(\frac{1}{2} T+c_{0}\right) H\right.}-\mathrm{e}^{i\left(t-\left(\frac{1}{2} T+c_{0}\right)\right) H \leftarrow}\right) \mathrm{e}^{-i\left(\frac{1}{2} T-c_{0}\right) H \leftarrow \tilde{f}}\right|^{2} \\
\leq\left(c_{0}-\frac{1}{2}+\frac{1}{2} m(T)\right) \\
\quad \times \sup _{\frac{1}{2}(1-m(T))-c_{0} \leq \sigma \leq 0}\left\|\left(\mathrm{e}^{i \sigma H}-\mathrm{e}^{i \sigma H \leftarrow}\right) \mathrm{e}^{-i\left(\frac{1}{2} T-c_{0}\right) H \leftarrow \tilde{f} t \mathrm{~d} \omega}\right\|_{H^{1}\left(\mathbb{R},\left(L^{2}\left(S^{2}\right)\right)^{4}\right)}
\end{array}
$$

We estimate

$$
\begin{aligned}
\|\left(\mathrm{e}^{i \sigma H}\right. & \left.-\mathrm{e}^{i \sigma H \leftarrow}\right) \mathrm{e}^{-i\left(\frac{1}{2} T-c_{0}\right) H \leftarrow \tilde{f}} \|_{H^{1}\left(\mathbb{R},\left(L^{2}\left(S^{2}\right)\right)^{4}\right)} \\
& \leq \int_{\sigma}^{0}\left\|\mathrm{e}^{i s H}\left(H-H_{\leftarrow}\right) \mathrm{e}^{i\left(\sigma-s-\frac{1}{2} T+c_{0}\right) H_{\leftarrow}} \tilde{f}\right\|_{H^{1}\left(\mathbb{R},\left(L^{2}\left(S^{2}\right)\right)^{4}\right)} \mathrm{d} s=: I_{\sigma} .
\end{aligned}
$$

We have

$$
\begin{aligned}
I_{\sigma} \leq \int_{\sigma}^{0}\left(\left\|D_{\widehat{r}}\left(P_{\omega}+W\right) \mathrm{e}^{i\left(\sigma-s-\frac{1}{2} T+c_{0}\right) H \leftarrow \tilde{f}}\right\|\right. \\
\left.+\left\|\left(P_{\omega}+W\right) \mathrm{e}^{i\left(\sigma-s-\frac{1}{2} T+c_{0}\right) H \leftarrow \tilde{f}}\right\|\right) \mathrm{d} s .
\end{aligned}
$$

We have

$$
\begin{aligned}
\Gamma^{1} D_{\widehat{r}}\left(P_{\omega}+W\right) \mathrm{e}^{i\left(\sigma-s-\frac{1}{2} T+c_{0}\right) H_{\leftarrow}} \tilde{f}=\left[\Gamma^{1} D_{\widehat{r}}, P_{\omega}+W\right] \mathrm{e}^{i\left(\sigma-s-\frac{1}{2} T+c_{0}\right) H_{\leftarrow}} \tilde{f} \\
+\left(P_{\omega}+W\right) \mathrm{e}^{i\left(\sigma-s-\frac{1}{2} T+c_{0}\right) H_{\leftarrow}} \Gamma^{1} D_{\widehat{r}} \tilde{f} .
\end{aligned}
$$

The last term equals

$$
\begin{aligned}
& \left(P_{\omega}+W\right) \mathrm{e}^{i\left(\sigma-s-\frac{1}{2} T+c_{0}\right) H \leftarrow} \Gamma^{1}\left(\chi_{R}\right)^{\prime}\left(\Omega_{\leftarrow}^{-} f\right)^{N} \\
& \quad+\left(P_{\omega}+W\right) \mathrm{e}^{i\left(\sigma-s-\frac{1}{2} T+c_{0}\right) H_{\leftarrow}}\left(\Omega_{\leftarrow}^{-} H f\right)_{R}^{N} .
\end{aligned}
$$

The first term can be treated in a similar manner. Using (9.8), (9.9) as well as

$$
\operatorname{supp} \mathrm{e}^{i\left(\sigma-s-\frac{1}{2} T+c_{0}\right) H \leftarrow \tilde{f} \subset\left(-\infty, R+\sigma-s-\frac{1}{2} T+c_{0}\right) \times[0,2 \pi] \times[0, \pi],}
$$

we can push further the estimate $(9.7)\left(0 \geq \sigma \geq \frac{1}{2}(1-m(T))-c_{0}\right)$ :

$$
\begin{aligned}
I_{\sigma} & \lesssim \int_{\sigma}^{0} \mathrm{e}^{\kappa_{+}\left(R+\sigma-s-\frac{1}{2} T\right)}(N+1)\left(\left\|\Omega_{\leftarrow}^{-} f\right\|+\left\|\Omega_{\leftarrow}^{-} H f\right\|\right) \mathrm{d} s \\
& \leq \frac{1}{\kappa_{+}} \mathrm{e}^{\kappa_{+}\left(R+c_{0}-\frac{1}{2} T\right)}(N+1)\left(\left\|\Omega_{\leftarrow}^{-} f\right\|+\left\|\Omega_{\leftarrow}^{-} H f\right\|\right)=: E(T) \longrightarrow 0, \quad T \rightarrow \infty .
\end{aligned}
$$

Choosing $m(T)=\min \left(\frac{1}{2} T, E(T)^{-\frac{1}{2}}\right)$ in $(9.6)$ we find $I_{3} \rightarrow 0, T \rightarrow \infty$. This concludes the proof of the lemma. 


\subsection{Proof of Proposition 9.1}

We start with the following lemma which analyzes the frequencies in $\not_{S^{2}}$ and $D_{\widehat{r}}$ of $U_{\leftarrow}(t, s) f, f \in \mathcal{H}^{1}$ :

LEMMA 9.3. - Let $f \in \mathcal{H}^{1}$, supp $f \subset\left[R_{1}, R_{2}\right] \times[0,2 \pi] \times[0, \pi]$. Then we have uniformly in $0 \leq t \leq s$ :

(i) $\left\|\not D_{S^{2}} U_{\leftarrow}(t, s) f\right\|_{\mathscr{H}_{t}} \leq C\left(R_{1}, R_{2}\right)\|f\|_{\mathscr{H}^{1}}$,

(ii) $\left\|D_{\widehat{r}} U_{\leftarrow}(t, s) f\right\|_{\mathscr{H}_{t}} \leq C\left(R_{1}, R_{2}\right) \mathrm{e}^{\kappa_{+} s}\|f\|_{\mathcal{H}^{1}}$.

Proof. - Let $u=U_{\leftarrow}(t, s) f$. Recall the explicit formula for $u$ from Section 8.4. We first show (i). We note:

$$
1-\dot{\widehat{z}}(t, \theta) \geq 1, \quad 1+\dot{\widehat{z}}(t, \theta) \gtrsim \mathrm{e}^{-2 \kappa_{+} t} .
$$

This follows from (3.53). We then claim that

$$
\left|\frac{\partial \widehat{\tau}}{\partial \theta}(\widehat{r}, \theta)\right| \lesssim 1 \quad \text { uniformly in } \widehat{r}, \theta \text {. }
$$

Indeed from

$$
\begin{gathered}
\widehat{z}(\widehat{\tau}(\widehat{r}, \theta), \theta)+\widehat{\tau}(\widehat{r}, \theta)=\widehat{r} \quad \text { follows with (9.10), } \\
\left|\frac{\partial \widehat{\tau}}{\partial \theta}(\widehat{r}, \theta)\right|=\left|\frac{\partial \widehat{z} / \partial \theta}{1+\dot{\grave{z}}}\right| \lesssim\left|\mathrm{e}^{2 \kappa_{+} \widehat{\tau}(\widehat{r}, \theta)} \frac{\partial \widehat{z}}{\partial \theta}\right| \lesssim 1
\end{gathered}
$$

It follows $(j=2,3)$ :

$$
\begin{aligned}
& \left|\partial_{\theta} f_{j}(\widehat{r}+t+s-2 \widehat{\tau}(\widehat{r}+t, \theta), \omega)\right| \\
& \lesssim\left|\left(\partial_{\theta} f_{j}\right)(\widehat{r}+t+s-2 \widehat{\tau}(\widehat{r}+t, \theta), \omega)\right| \\
& \quad+\left|\left(\partial_{\widehat{r}} f_{j}\right)(\widehat{r}+t+s-2 \widehat{\tau}(\widehat{r}+t, \theta), \omega)\right| .
\end{aligned}
$$

We next claim:

$$
\left|\partial_{\theta} \sqrt{\frac{1-\dot{\hat{z}}(\widehat{\tau}(\widehat{r}+t, \theta), \theta)}{1+\dot{\hat{z}}(\widehat{\tau}(\widehat{r}+t, \theta), \theta)}}\right| \lesssim \sqrt{\frac{1-\dot{\grave{z}}(\widehat{\tau}(\widehat{r}+t, \theta), \theta)}{1+\dot{\hat{z}}(\widehat{\tau}(\widehat{r}+t, \theta), \theta)}} .
$$

Indeed

$$
\begin{aligned}
& \left|\partial_{\theta} \sqrt{\frac{1-\dot{\hat{z}}(\widehat{\tau}(\widehat{r}+t, \theta), \theta)}{1+\dot{\hat{z}}(\widehat{\tau}(\widehat{r}+t, \theta), \theta)}}\right|=\left|\frac{1}{1-\dot{\widehat{z}}} \frac{(\ddot{\vec{z}} \partial \widehat{\tau} / \partial \theta+\partial \dot{\widehat{z}} / \partial \theta)}{1+\dot{\hat{z}}} \sqrt{\frac{1-\dot{\widehat{z}}(\widehat{\tau}(\widehat{r}+t, \theta), \theta)}{1+\dot{\widehat{z}}(\widehat{\tau}(\widehat{r}+t, \theta), \theta)}}\right| \\
& \lesssim \sqrt{\frac{1-\dot{\widehat{z}}(\widehat{\tau}(\widehat{r}+t, \theta), \theta)}{1+\dot{\widehat{z}}(\widehat{\tau}(\widehat{r}+t, \theta), \theta)}},
\end{aligned}
$$


where we have used (9.10) and the uniform boundedness of $\ddot{\widehat{z}} /(1+\dot{\widehat{z}}), \partial \widehat{\tau} / \partial \theta$ and $\partial \dot{\widehat{z}} / \partial \theta /(1+\dot{\widehat{z}})$. It follows

$$
\begin{aligned}
\left\|\not D_{S^{2}} U_{\leftarrow}(t, s) f\right\|_{\mathcal{H}_{t}} & \lesssim\left\|U_{\leftarrow}(t, s)\left(\partial_{\theta} f\right)\right\|+\left\|U_{\leftarrow}(t, s) \frac{1}{\sin \theta}\left(\partial_{\varphi} f\right)\right\| \\
& +\left\|U_{\leftarrow}(t, s)\left(\partial_{\widehat{r}} f\right)\right\|+\|f\| \\
& \leq C\left(R_{1}, R_{2}\right)\|f\|_{\mathcal{H}^{1}} .
\end{aligned}
$$

Let us now show (ii). We first claim that on $\operatorname{supp} f_{j}(\widehat{r}+t+s-2 \widehat{\tau}(\widehat{r}+t, \theta)$, $\omega)$ we have for $s$ sufficiently large

$$
\left|\frac{\partial \widehat{\tau}}{\partial \widehat{r}}(\widehat{r}+t, \theta)\right| \lesssim \frac{1}{|\widehat{r}+t|}
$$

Indeed from

$$
\widehat{z}(\widehat{\tau}(\widehat{r}+t, \theta), \theta)+\widehat{\tau}(\widehat{r}+t, \theta)=\widehat{r}+t
$$

follows

$$
\left|\frac{\partial \widehat{\tau}}{\partial \widehat{r}}\right| \leq \frac{1}{|\dot{\vec{z}}(\widehat{\tau}(\widehat{r}+t, \theta), \theta)+1|} \lesssim \frac{1}{|\widehat{r}+t|},
$$

where we have used (8.19) and the fact that

$$
\widehat{r}+t \rightarrow 0, s \rightarrow \infty \quad \text { on } \quad \operatorname{supp} f_{j}(\widehat{r}+t+s-2 \widehat{\tau}(\widehat{r}+t, \theta), \omega) \text {. }
$$

We next note that on $\operatorname{supp} f_{j}(\widehat{r}+t+s-2 \widehat{\tau}(\widehat{r}+t, \theta), \omega)$ we have

$$
|\widehat{r}+t| \gtrsim \mathrm{e}^{-\kappa_{+} s}
$$

This follows from (8.18). We now estimate on $\operatorname{supp} f_{j}(\widehat{r}+t+s-2 \widehat{\tau}(\widehat{r}+t, \theta), \omega)$ :

$$
\begin{aligned}
& \left|\partial_{\widehat{r}}\left(\left(\frac{1-\dot{\widehat{z}}}{1+\dot{\grave{z}}}\right)^{\frac{1}{2}}(\widehat{\tau}(\widehat{r}+t, \theta), \theta)\right)\right| \\
& =\left|\left(\frac{1-\dot{\widehat{z}}}{1+\dot{\widehat{z}}}(\widehat{\tau}(\widehat{r}+t, \theta), \theta)\right)^{\frac{1}{2}} \frac{\ddot{\widehat{z}}(\widehat{\tau}(\widehat{r}+t, \theta), \theta) \partial_{\widehat{r}} \widehat{\tau}(\widehat{r}+t, \theta)}{(1+\dot{\widehat{z}})(1-\dot{\widehat{z}})}\right| \\
& \quad \mathrm{e}^{\kappa+s}\left(\frac{1-\dot{\widehat{z}}}{1+\dot{\grave{z}}}\right)^{\frac{1}{2}}(\widehat{\tau}(\widehat{r}+t, \theta), \theta) .
\end{aligned}
$$

In the same way we estimate

$$
\begin{aligned}
& \left|\partial_{\widehat{r}} f_{j}(\widehat{r}+t+s-2 \widehat{\tau}(\widehat{r}+t, \theta), \omega)\right| \\
& \leq\left|\left(\partial_{\widehat{r}} f_{j}\right)(\widehat{r}+t+s-2 \widehat{\tau}(\widehat{r}+t, \theta), \omega)\left(1-2 \partial_{\widehat{r}} \widehat{\tau}\right)\right| \\
& \quad \lesssim \mathrm{e}^{\kappa_{+} s}\left|\left(\partial_{\widehat{r}} f_{j}\right)(\widehat{r}+t+s-2 \widehat{\tau}(\widehat{r}+t, \theta), \omega)\right|
\end{aligned}
$$

The estimates (9.13) and (9.14) give (ii). 
Proof of Proposition 9.1. - Let $\epsilon>0$. We first note that

$$
\mathcal{J}\left(\widehat{r}+t_{\epsilon}\right) U\left(t_{\epsilon}, T\right) f=\mathcal{J}\left(\widehat{r}+t_{\epsilon}\right) G_{t_{\epsilon}}\left(g^{T}\right),
$$

where $G_{t_{\epsilon}}\left(g^{T}\right)$ is the solution at time $t_{\epsilon}$ of the characteristic problem (7.31) with data $g^{T}$. In the same way we denote $G_{t_{\epsilon}}\left(g_{\leftarrow, R}^{T, N}\right)$ the solution at time $t_{\epsilon}$ of the characteristic Cauchy problem (7.31) with data $g_{\leftarrow, R}^{T, N}$. We estimate:

$$
\begin{aligned}
& \left\|\mathcal{J}\left(\widehat{r}+t_{\epsilon}\right)\left(U\left(t_{\epsilon}, T\right) f-U_{\leftarrow}\left(t_{\epsilon}, T\right) \Omega_{\leftarrow}^{-} f\right)\right\|_{\mathscr{H}_{t_{\epsilon}}} \\
& \leq\left\|\mathcal{\partial}\left(\widehat{r}+t_{\epsilon}\right)\left(G_{t_{\epsilon}}\left(g^{T}\right)-G_{t_{\epsilon}}\left(g_{\leftarrow, R}^{T, N}\right)\right)\right\|_{\mathscr{H}_{t_{\epsilon}}} \\
& +\left\|\mathcal{J}\left(\widehat{r}+t_{\epsilon}\right)\left(G_{t_{\epsilon}}\left(g_{\leftarrow, R}^{T, N}\right)-U_{\leftarrow}\left(t_{\epsilon}, T\right)\left(\Omega_{\leftarrow}^{-} f\right)_{R}^{N}\right)\right\|_{\mathcal{H}_{t_{\epsilon}}} \\
& +\left\|\left(\Omega_{\leftarrow}^{-} f\right)_{R}^{N}-\Omega_{\leftarrow}^{-} f\right\| \\
& \leq\left(\int_{0}^{\infty} \int_{S^{2}}\left|g^{T}-g_{\leftarrow}^{T}\right|^{2} \mathrm{~d} t \mathrm{~d} \omega\right)^{\frac{1}{2}}+\left(\int_{0}^{\infty} \int_{S^{2}}\left|g_{\leftarrow}^{T}-g_{\leftarrow, R}^{T, N}\right|^{2} \mathrm{~d} t \mathrm{~d} \omega\right)^{\frac{1}{2}} \\
& +\left\|\mathcal{J}\left(\widehat{r}+t_{\epsilon}\right) U\left(t_{\epsilon}, \frac{1}{2} T+c_{0}\right)\left(\Phi^{R, N}\left(\frac{1}{2} T+c_{0}, .\right)-\Phi_{\leftarrow}^{R, N}\left(\frac{1}{2} T+c_{0}, .\right)\right)\right\|_{\mathcal{H}_{t_{\epsilon}}} \\
& +\left\|\left(U\left(t_{\epsilon}, \frac{1}{2} T+c_{0}\right)-U_{\leftarrow}\left(\frac{1}{2} T+c_{0}, .\right)\right) \Phi_{\leftarrow}^{R, N}\left(\frac{1}{2} T+c_{0}, .\right)\right\|_{\mathcal{H}_{t_{\epsilon}}} \\
& +\left\|\left(\Omega_{\leftarrow}^{-} f\right)_{R}^{N}-\Omega_{\leftarrow}^{-} f\right\| \\
& \leq\left(\int_{0}^{\infty} \int_{S^{2}}\left|g^{T}-g_{\leftarrow}^{T}\right|^{2} \mathrm{~d} t \mathrm{~d} \omega\right)^{\frac{1}{2}}+2\left\|\left(\Omega_{\leftarrow}^{-} f\right)_{R}^{N}-\Omega_{\leftarrow}^{-} f\right\| \\
& +\left\|\mathcal{J}\left(\widehat{r}+t_{\epsilon}\right) U\left(t_{\epsilon}, \frac{1}{2} T+c_{0}\right)\left(\Phi^{R, N}\left(\frac{1}{2} T+c_{0}, .\right)-\Phi_{\leftarrow}^{R, N}\left(\frac{1}{2} T+c_{0}, .\right)\right)\right\|_{\mathcal{H}_{t_{\epsilon}}} \\
& +\left\|\left(U\left(t_{\epsilon}, \frac{1}{2} T+c_{0}\right)-U_{\leftarrow}\left(t_{\epsilon}, \frac{1}{2} T+c_{0}\right)\right) \Phi_{\leftarrow}^{R, N}\left(\frac{1}{2} T+c_{0}, .\right)\right\|_{\mathcal{H}_{t_{\epsilon}}} .
\end{aligned}
$$

We now fix $R, N$ such that

$$
2\left\|\left(\Omega_{\leftarrow}^{-} f\right)_{R}^{N}-\Omega_{\leftarrow}^{-} f\right\|<\frac{1}{4} \epsilon .
$$

In order to estimate the last term in (9.15) we want to use the Duhamel formula. Let $\tau_{T}$ and $\tau_{T}^{1}$ be defined by

$$
\tau_{T}-T+R=\widehat{z}\left(\tau_{T}, \theta\right) \Longrightarrow \tau_{T}=\frac{1}{2} T-\frac{1}{2} R+\theta\left(\mathrm{e}^{-\kappa_{+} T}\right)
$$

(resp. an analogous definition for $\tau_{T}^{1}$ with $R$ replaced by $R_{1}$ ). For the above implication we have used (3.54). We now observe that (see Figure 2)

$\triangleright$ if $s \geq \tau_{T}^{1}$ then

$$
\operatorname{supp} U_{\leftarrow}(s, T)\left(\Omega_{\leftarrow}^{-} f\right)_{R}^{N} \subset[\widehat{z}(s, \theta), s+R-T],
$$

$\triangleright$ if $\tau_{T}^{1} \geq s \geq \tau_{T}$ then

$$
\operatorname{supp} U_{\leftarrow}(s, T)\left(\Omega_{\leftarrow}^{-} f\right)_{R}^{N} \subset\left[\widehat{z}(s, \theta), \max \left(s+R-T,-s+2 \tau_{T}^{1}-T+R_{1}\right)\right],
$$

$\triangleright$ if $\tau_{T} \geq s$ then

$$
\operatorname{supp} U_{\leftarrow}(s, T)\left(\Omega_{\leftarrow}^{-} f\right)_{R}^{N} \subset\left[-s+2 \tau_{T}-T+R,-s+2 \tau_{T}^{1}-T+R_{1}\right] .
$$




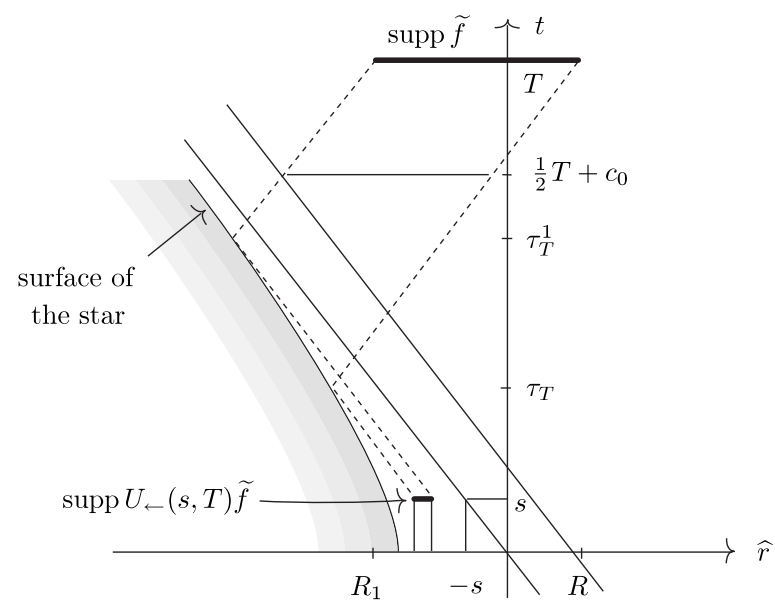

FiguRE 2. The support of $\left(\Omega_{\leftarrow}^{-} f\right)_{R}^{N}$ transported by the asymptotic propagator $U_{\leftarrow}(s, T)$.

We have to distinguish the cases $s \in\left[\tau_{T}, \tau_{T}^{1}\right]$ and $s \in[0, T] \backslash\left[\tau_{T}, \tau_{T}^{1}\right]$.

If $s \in[0, T] \backslash\left[\tau_{T}, \tau_{T}^{1}\right]$, then $U_{\leftarrow}(s, T)\left(\Omega_{\leftarrow}^{-} f\right)_{R}^{N}$ is zero on the boundary, in particular

$$
U_{\leftarrow}(s, T)\left(\Omega_{\leftarrow}^{-} f\right)_{R}^{N} \in D\left(H_{s}\right), \quad \forall s \in[0, T] \backslash\left[\tau_{T}, \tau_{T}^{1}\right] .
$$

Here we have used Lemma 9.3 to establish the necessary regularity of $U_{\leftarrow}(s, T)\left(\Omega_{\leftarrow}^{-} f\right)_{R}^{N}$. But we have

$$
U_{\leftarrow}(s, T)\left(\Omega_{\leftarrow}^{-} f\right)_{R}^{N} \notin D\left(H_{s}\right), \quad \forall s \in\left[\tau_{T}, \tau_{T}^{1}\right] .
$$

Let

$$
M(t, \theta)=\widehat{w}(t, \theta) \widehat{w}_{0}(t, \theta) \widehat{\Gamma}^{4}\left(\widehat{w}^{-1} \widehat{\Gamma}^{4}+\frac{Z_{1}}{i}-\frac{Z_{2}}{i} \widehat{\Gamma}^{2}\right) \widehat{\Gamma}^{4}\left(\widehat{w}_{0}^{-1} \widehat{\Gamma}^{4}-\frac{Z_{1}}{i}\right)
$$

with $Z_{1}=\dot{\grave{z}}(t, \theta)+1$ and $Z_{2}=\left(\partial_{\theta} \widehat{z}\right)(t, \theta) a_{0}(\widehat{z}(t, \theta), \theta) h^{2}(\widehat{z}(t, \theta), \theta)$. The coefficients in $\widehat{\Gamma}^{2}$ have to be evaluated at $(\widehat{z}(t, \theta), \theta)$. We first note that a matrix of type

$$
V=\widehat{\Gamma}^{4}\left(\widehat{w}^{-1} \widehat{\Gamma}^{4}-\frac{Z_{1}}{i}+\frac{Z_{2}}{i} \widehat{\Gamma}^{2}\right)
$$

is invertible. Indeed an elementary calculation using the anticommutation relations for Dirac matrices gives

$$
V \widehat{\Gamma}^{4}\left(\widehat{w}^{-1} \widehat{\Gamma}^{4}+\frac{Z_{1}}{i}-\frac{Z_{2}}{i} \widehat{\Gamma}^{2}\right)=\widehat{w}^{-2}+Z_{1}^{2}-Z_{2}^{2} .
$$

Let

$$
A=\widehat{\Gamma}^{4}\left(-\dot{\widehat{z}}-\Gamma^{1}+i \widehat{w}^{-1} \widehat{\Gamma}^{4}+Z_{2} \widehat{\Gamma}^{2}\right) .
$$

Then $V$ is an isomorphism from $\operatorname{Ker} A$ to $\mathcal{K}=\left\{\left(\Psi_{1}, 0,0, \Psi_{4}\right) \in \mathbb{C}^{4}\right\}$. For dimensional reasons we only have to show $V \operatorname{Ker} A \subset \mathcal{K}$, which follows from

$$
P_{2,3} V \Psi=P_{2,3} \widehat{\Gamma}^{4}\left(\widehat{w}^{-1} \widehat{\Gamma}^{4}-\frac{\dot{\bar{z}}}{i}-\frac{\Gamma^{1}}{i}+\frac{Z_{2}}{i} \widehat{\Gamma}^{2}\right) \Psi=0
$$


for $\Psi \in \operatorname{Ker} A$. This shows that $M(s, \theta) U_{\leftarrow}(s, T)\left(\Omega_{\leftarrow}^{-} f\right)_{R}^{N}$ fulfills the boundary conditions for $H_{s}$. We have

$$
M=1+\frac{Z_{2} \widehat{w}}{i} \widehat{\Gamma}^{2} \widehat{\Gamma}^{4}+Z_{2} Z_{1} \widehat{w} \widehat{w}_{0} \widehat{\Gamma}^{2}+\frac{Z_{1}}{i}\left(\widehat{w}-\widehat{w}_{0}\right) \widehat{\Gamma}^{4}+Z_{1}^{2} \widehat{w} \widehat{w}_{0} .
$$

By (3.53) we have

$$
\widehat{w} \lesssim \mathrm{e}^{\kappa_{+} t}, \quad \widehat{w}_{0} \lesssim \mathrm{e}^{\kappa_{+} t}
$$

We estimate:

$$
\left|\widehat{w}-\widehat{w}_{0}\right| \leq \widehat{w} \widehat{w}_{0}\left(1-\dot{\vec{z}}^{2}\right)^{\frac{1}{2}}\left|\left(1+\frac{\left(\partial_{\theta} \widehat{z}\right)^{2} a_{0}^{2} h^{4}-2\left(\partial_{\theta} \widehat{z}\right) \ell^{\prime} a_{0}^{2} h^{4}}{1-\dot{\widehat{z}}^{2}}\right)^{\frac{1}{2}}-1\right| .
$$

As

$$
\left|\frac{\left(\partial_{\theta} \widehat{z}\right)^{2} a_{0}^{2} h^{4}-2\left(\partial_{\theta} \widehat{z}\right) \ell^{\prime} a_{0}^{2} h^{4}}{1-\dot{\widehat{z}}^{2}}\right| \lesssim \mathrm{e}^{-2 \kappa_{+} t}
$$

we obtain

$$
\left|\widehat{w}-\widehat{w}_{0}\right| \lesssim \mathrm{e}^{-\kappa_{+} t}
$$

This entails

$$
M(t)=1+\theta\left(\mathrm{e}^{-2 \kappa_{+} t}\right), \quad \frac{\mathrm{d}}{\mathrm{d} t} M(t)=\theta\left(\mathrm{e}^{-2 \kappa+t}\right)
$$

We write

$$
\begin{aligned}
I:= & \left(U\left(t_{\epsilon}, \frac{1}{2} T+c_{0}\right)-U_{\leftarrow}\left(t_{\epsilon}, \frac{1}{2} T+c_{0}\right)\right) \Phi_{\leftarrow}^{R, N}\left(\frac{1}{2} T+c_{0}\right) \\
= & U\left(t_{\epsilon}, \tau_{T}\right)\left(U\left(\tau_{T}, \frac{1}{2} T+c_{0}\right)-U_{\leftarrow}\left(\tau_{T}, \frac{1}{2} T+c_{0}\right)\right) \Phi_{\leftarrow}^{R, N}\left(\frac{1}{2} T+c_{0}\right) \\
& \quad \quad+\left(U\left(t_{\epsilon}, \tau_{T}\right)-U_{\leftarrow}\left(t_{\epsilon}, \tau_{T}\right)\right) U_{\leftarrow}\left(\tau_{T}, \frac{1}{2} T+c_{0}\right) \Phi_{\leftarrow}^{R, N}\left(\frac{1}{2} T+c_{0}\right) \\
& =: I_{1}+I_{2} .
\end{aligned}
$$

Recalling that $U_{\leftarrow}\left(s, \frac{1}{2} T+c_{0}\right) \Phi_{\leftarrow}^{R, N}\left(\frac{1}{2} T+c_{0}\right)=U_{\leftarrow}(s, T)\left(\Omega_{\leftarrow}^{-} f\right)_{R}^{N}$ and using (9.16) we can estimate the second term using the Duhamel formula:

$$
\begin{aligned}
\left\|I_{2}\right\| & \leq \int_{t_{\epsilon}}^{\tau_{T}}\left\|\left(P_{\omega}+W\right) U_{\leftarrow}(s, T)\left(\Omega_{\leftarrow}^{-} f\right)_{R}^{N}\right\|_{\mathcal{H}_{s}} \mathrm{~d} s \\
& \leq C\left(R_{1}, R\right) \int_{t_{\epsilon}}^{\tau_{T}} \mathrm{e}^{-\kappa_{+} s} \mathrm{~d} s\left\|\left(\Omega_{\leftarrow}^{-} f\right)_{R}^{N}\right\|_{\mathcal{H}^{1}} \\
& \leq C\left(R_{1}, R\right)(N+1)\left(\left\|\Omega_{\leftarrow}^{-} f\right\|+\left\|\Omega_{\leftarrow}^{-} H f\right\|\right) \frac{1}{\kappa_{+}}\left(\mathrm{e}^{-\kappa_{+} t_{\epsilon}}-\mathrm{e}^{-\kappa_{+} \tau_{T}}\right) .
\end{aligned}
$$

Here we have used Lemma 9.3. To estimate the first term we write

$$
\begin{aligned}
& \left(U\left(\tau_{T}, \frac{1}{2} T+c_{0}\right)-U_{\leftarrow}\left(\tau_{T}, \frac{1}{2} T+c_{0}\right)\right) \Phi_{\leftarrow}^{R, N}\left(\frac{1}{2} T+c_{0}\right) \\
& =\left(U\left(\tau_{T}, \frac{1}{2} T+c_{0}\right) M(T)-M\left(\tau_{T}\right) U_{\leftarrow}\left(\tau_{T}, \frac{1}{2} T+c_{0}\right)\right) \Phi_{\leftarrow}^{R, N}\left(\frac{1}{2} T+c_{0}\right) \\
& \quad+\vartheta\left(\mathrm{e}^{-\kappa_{+} T}\right),
\end{aligned}
$$


where we have used (9.19). Now we can use the Duhamel formula:

$$
\begin{gathered}
\left\|\left(U\left(\tau_{T}, \frac{1}{2} T+c_{0}\right) M(T)-M\left(\tau_{T}\right) U_{\leftarrow}\left(\tau_{T}, \frac{1}{2} T+c_{0}\right)\right) \Phi_{\leftarrow}^{R, N}\left(\frac{1}{2} T+c_{0}\right)\right\| \\
\leq \int_{\tau_{T}}^{\frac{1}{2} T+c_{0}}\left\|U\left(\tau_{T}, s\right)\left(H M(s)-M(s) H_{\leftarrow}\right) U_{\leftarrow}(s, T)\left(\Omega_{\leftarrow}^{-} f\right)_{R}^{N}\right\| \mathrm{d} s \\
\quad+\int_{\tau_{T}}^{\frac{1}{2} T+c_{0}}\left\|U\left(\tau_{T}, s\right) \frac{\mathrm{d} M(s)}{\mathrm{d} s} U_{\leftarrow}(s, T)\left(\Omega_{\leftarrow}^{-} f\right)_{R}^{N}\right\| \mathrm{d} s=: I_{2}^{1}+I_{2}^{2} .
\end{gathered}
$$

To be more precise we should have used the operators $H_{s}$ and $H_{\leftarrow, s}$ in the above formula. But as $U_{\leftarrow}(s, T)\left(\Omega_{\leftarrow}^{-} f\right)_{R}^{N}$ is a smooth function we have:

$$
\begin{aligned}
M(s) H_{\leftarrow, s} U_{\leftarrow, s}(s, T)\left(\Omega_{\leftarrow}^{-} f\right)_{R}^{N}=\Gamma^{1} D_{\widehat{r}} M U_{\leftarrow}(s, T)\left(\Omega_{\leftarrow}^{-} f\right)_{R}^{N} \\
+\left[M, \Gamma^{1} D_{\widehat{r}}\right] U_{\leftarrow}(s, T)\left(\Omega_{\leftarrow}^{-} f\right)_{R}^{N} .
\end{aligned}
$$

This will be used below. By (9.19) we have

$$
I_{2}^{2} \lesssim \mathrm{e}^{-\kappa_{+} T}\left\|\Omega_{\leftarrow}^{-} f\right\|
$$

Let us now estimate $I_{2}^{1}$ :

$$
\begin{aligned}
I_{2}^{1} \leq \int_{\tau_{T}}^{\frac{1}{2} T+c_{0}} & \left\|U\left(\tau_{T}, s\right)\left(H-H_{\leftarrow}\right) M U_{\leftarrow}(s, T)\left(\Omega_{\leftarrow}^{-} f\right)_{R}^{N}\right\| \mathrm{d} s \\
& \quad+\int_{\tau_{T}}^{\frac{1}{2} T+c_{0}}\left\|U\left(\tau_{T}, s\right)\left[M, H_{\leftarrow}\right] U_{\leftarrow}(s, T)\left(\Omega_{\leftarrow}^{-} f\right)_{R}^{N}\right\| \mathrm{d} s=: I_{a}+I_{b} .
\end{aligned}
$$

We have $\left[M, H_{\leftarrow}\right]=\left[M, \Gamma^{1}\right] D_{\widehat{r}}$. Recalling that $\Gamma^{1}=\left(\alpha \widehat{\Gamma}^{1}+\beta \widehat{\Gamma}^{2}\right)(\widehat{z}(s, \theta), \theta)$ we find

$$
\left[Z_{2} \widehat{w} \widehat{\Gamma}^{2} \widehat{\Gamma}^{4}, \Gamma^{1}\right]=Z_{2} \widehat{w} \beta\left[\widehat{\Gamma}^{2} \widehat{\Gamma}^{4}, \widehat{\Gamma}^{2}\right] .
$$

It follows

$$
\left[M(s), \Gamma^{1}\right]=\Theta\left(\mathrm{e}^{-3 \kappa+s}\right) .
$$

Therefore we can estimate $I_{b}$ using Lemma 9.3 (ii):

$$
\begin{aligned}
I_{b} & \leq C\left(R_{1}, R\right) \int_{\tau_{T}}^{\frac{1}{2} T+c_{0}} \mathrm{e}^{-3 \kappa_{+} s} \mathrm{e}^{\kappa_{+} T}(N+1)\left(\left\|\Omega_{\leftarrow}^{-} f\right\|+\left\|\Omega_{\leftarrow}^{-} H f\right\|\right) \mathrm{d} s \\
& \leq C\left(R_{1}, R\right) \mathrm{e}^{-\frac{\kappa_{+}}{2} T}(N+1)\left(\left\|\Omega_{\leftarrow}^{-} f\right\|+\left\|\Omega_{\leftarrow}^{-} H f\right\|\right) .
\end{aligned}
$$

We now estimate $I_{a}$ using Lemma 9.3 (i):

$$
\begin{aligned}
I_{a} & \leq \int_{\tau_{T}}^{\frac{1}{2} T+c_{0}}\left\|\left(P_{\omega}+W\right) M U_{\leftarrow}(s, T)\left(\Omega_{\leftarrow}^{-} f\right)_{R}^{N}\right\| \mathrm{d} s \\
& \leq C\left(R_{1}, R\right) \int_{\tau_{T}}^{\frac{1}{2} T+c_{0}} \mathrm{e}^{-\kappa_{+} s}\left\|\left(\Omega_{\leftarrow}^{-} f\right)_{R}^{N}\right\|_{\mathcal{H}^{1}} \mathrm{~d} s \\
& \leq C\left(R_{1}, R\right) \mathrm{e}^{-\kappa_{+} \frac{1}{2} T}(N+1)\left(\left\|\Omega_{\leftarrow}^{-} f\right\|+\left\|\Omega_{\leftarrow}^{-} H f\right\|\right) .
\end{aligned}
$$


Here we have used that $D_{\theta} M=\Theta\left(\mathrm{e}^{-2 \kappa_{+} t}\right)$. Putting everything together we find: (9.24) $\|I\| \leq C\left(R_{1}, R\right) \mathrm{e}^{-\kappa_{+} t_{\epsilon}}(N+1)\left(\left\|\Omega_{\leftarrow}^{-} f\right\|+\left\|\Omega_{\leftarrow}^{-} H f\right\|\right) \quad$ uniformly in $T$ large.

We fix $t_{\epsilon}$ large enough such that the term on the R.H.S. of (9.24) is controlled by $\epsilon / 4$ uniformly in $T$ large. For $T$ sufficiently large we can estimate the first and the third terms in (9.15) using Lemmas 9.1 and 9.2:

$$
\begin{gathered}
\left(\int_{0}^{\infty} \int_{S^{2}}\left|g^{T}-g_{\leftarrow}^{T}\right|^{2} \mathrm{~d} t \mathrm{~d} \omega\right)^{\frac{1}{2}}<\frac{1}{4} \epsilon, \\
\left\|\mathcal{J}\left(\widehat{r}+t_{\epsilon}\right) U\left(t_{\epsilon}, \frac{1}{2} T+c_{0}\right)\left(\Phi^{R, N}\left(\frac{1}{2} T+c_{0}, .\right)-\Phi_{\leftarrow}^{R, N}\left(\frac{1}{2} T+c_{0}, .\right)\right)\right\|_{\mathcal{H}_{t_{\epsilon}}}<\frac{1}{4} \epsilon .
\end{gathered}
$$

This concludes the proof of the proposition. 


\section{CHAPTER 10}

\section{PROPAGATION OF SINGULARITIES}

So far we have compared the full dynamics $U(s, T) f$ to the dynamics $U_{\leftarrow}(s, t) \Omega_{\leftarrow}^{-} f$ on the interval $\left[t_{\epsilon}, T\right]$. We will now replace the dynamics $U_{\leftarrow}\left(t_{\epsilon}, T\right) \Omega_{\leftarrow}^{-} f$ by the so called geometric optics approximation. We suppose for the whole chapter that

$$
f(\widehat{r}, \omega)=\mathrm{e}^{i n \varphi} f^{n}(\widehat{r}, \theta), \quad f^{n} \in C_{0}^{\infty}(\mathbb{R} \times[0, \pi]), \quad n \in \mathbb{Z}+\frac{1}{2} .
$$

Let

$$
\begin{aligned}
F_{t_{0}}^{T}(\widehat{r}, \omega):= & \frac{1}{\sqrt{-\kappa_{+}\left(\widehat{r}+t_{0}\right)}}\left(\widetilde{f}_{3}, 0,0,-\widetilde{f}_{2}\right) \\
& \quad \times\left(T+\frac{1}{\kappa_{+}} \ln \left(-\left(\widehat{r}+t_{0}\right)\right)-\frac{1}{\kappa_{+}} \ln \widehat{A}(\theta), \omega\right),
\end{aligned}
$$

where $\tilde{f}=\left(\Omega_{\leftarrow}^{-} f\right)_{R}^{N}$ (see Section 9.2). Note that

$$
\operatorname{supp} F_{t_{0}}^{T} \subset\left(-t_{0}-\left|\theta\left(\mathrm{e}^{-\kappa_{+} T}\right)\right|,-t_{0}\right) \times[0,2 \pi] \times[0, \pi]
$$

and that $F_{t_{0}}^{T}$ depends on $N, R$. All functions involved have fixed angular momentum, e.g. $F_{t_{0}}^{T}(\widehat{r}, \omega)=F_{t_{0}, n}^{T}(\widehat{r}, \theta) \mathrm{e}^{i n \varphi}$. The functions $F_{t_{0}}^{T}$ and $F_{t_{0}, n}^{T}$ will often be identified. We therefore fix now the angular momentum $\partial_{\varphi}=i n$ everywhere in the expression of $H$ :

$$
H=\Gamma^{1} D_{\widehat{r}}+\left(\begin{array}{cc}
M_{\theta} & 0 \\
0 & -M_{\theta}
\end{array}\right)+\frac{h^{2} a_{0}}{\sin \theta} \widehat{\Gamma}^{3} n+h^{2} c_{1}+h^{2} c_{2}^{\varphi} n+\widehat{V}_{\varphi} n+\widehat{V}_{1}^{\nu}+\frac{q Q r_{+}}{r_{+}^{2}+a^{2}}+\frac{a n}{r_{+}^{2}+a^{2}} .
$$

Here $\widehat{V}_{1}^{\nu}$ is obtained from $\widehat{V}_{1}$ by replacing $\widehat{\Gamma}^{4}$ by $\Gamma^{\nu}$. Recall that

$$
\left(\begin{array}{cc}
M_{\theta} & 0 \\
0 & -M_{\theta}
\end{array}\right)+\frac{h^{2} a_{0}}{\sin \theta} \widehat{\Gamma}^{3} n=\mathcal{U} h \sqrt{a_{0}} \not_{S^{2}}^{n} \sqrt{a_{0}} h \mathcal{U}^{*},
$$

where $\not_{S^{2}}^{n}$ is the restriction of $\nsupseteq_{S^{2}}$ to $\left\{u=\mathrm{e}^{i n \varphi} \widetilde{u}(\theta) ; \widetilde{u}(\theta) \in L^{2}\left(([0, \pi] ; \sin \theta \mathrm{d} \theta) ; \mathbb{C}^{4}\right)\right\}$. Therefore $H$ is a regular operator and the singularities in the expression of $H$ are coordinate singularities. We put

$$
\widehat{\mathcal{H}}=\left(L^{2}(\mathbb{R} \times[0, \pi] ; \mathrm{d} \widehat{r} \sin \theta \mathrm{d} \theta)\right)^{4} .
$$


Clearly

$$
\widehat{\mathscr{H}}=\bigoplus_{\ell} \widehat{\mathscr{H}}^{\ell}, \quad \widehat{\mathscr{H}}^{\ell}=\left(L^{2}(\mathbb{R})\right)^{4} \otimes_{4} Y_{\ell}, \quad Y_{\ell}=\left(\operatorname{span}\left\{\mathrm{e}^{2 i l \theta}\right\}\right)^{4} .
$$

For $\delta>0$ let $\phi_{\delta} \in C^{\infty}(\mathbb{R})$ with

$$
\phi_{\delta}(\widehat{r})= \begin{cases}1 & \widehat{r} \geq \delta \\ 0 & \widehat{r} \leq \frac{1}{2} \delta\end{cases}
$$

The aim of this chapter is to prove the following:

Proposition 10.1. - We have

$$
\begin{gathered}
\forall \epsilon>0, \exists N_{0}>0, R_{0}>0, \forall N \geq N_{0}, R \geq R_{0}, \exists t_{0}>0, \\
\forall t_{\epsilon} \geq t_{0}, \exists \delta=\delta\left(t_{\epsilon}, N, R\right), T_{0}=T_{0}\left(t_{\epsilon}, \delta, N, R\right), \forall T \geq T_{0}, \\
\left\|\partial U(0, T) f-\phi_{\delta}(.-\widehat{z}(0, \theta)) \mathrm{e}^{-i t_{\epsilon} H} F_{t_{\epsilon}}^{T}\right\| \leq \epsilon .
\end{gathered}
$$

Note that we can consider $\mathscr{J} U(0, T) f-\phi_{\delta}(.-\widehat{z}(0, \theta)) \mathrm{e}^{-i t_{\epsilon} H} F_{t_{\epsilon}}^{T}$ as an element of $\widehat{\mathcal{H}}$ and it is sufficient to show

$$
\left\|\partial U(0, T) f-\phi_{\delta}(.-\widehat{z}(0, \theta)) \mathrm{e}^{-i t_{\epsilon} H} F_{t_{\epsilon}}^{T}\right\|_{\widehat{\mathcal{H}}} \leq \epsilon .
$$

We will use the pseudodifferential calculus on $\Sigma_{1}=\mathbb{R}_{\widehat{r}} \times(0, \pi)$. We note $\xi$ the dual variable to $\widehat{r}$ and $q$ the dual variable to $\theta$. Let $S^{m}\left(\Sigma_{1}\right)$ be the space of symbols of order $m$ and $\Psi^{m}\left(\Sigma_{1}\right)$ be the space of pseudodifferential operators of order $m$ (see [31, Chapter XVIII]):

$$
\begin{aligned}
a(\widehat{r}, \theta, \xi, q) \in S^{m}\left(\Sigma_{1}\right) \Longleftrightarrow \forall \alpha & =\left(\alpha_{1}, \alpha_{2}\right), \beta=\left(\beta_{1}, \beta_{2}\right), \\
& \left|\partial_{\xi}^{\alpha_{1}} \partial_{q}^{\alpha_{2}} \partial_{\widehat{r}}^{\beta_{1}} \partial_{\theta}^{\beta_{2}} a(\widehat{r}, \theta, \xi, q)\right| \leq C_{\alpha, \beta}\langle(\xi, q)\rangle^{m-|\alpha|} .
\end{aligned}
$$

For a matrix $M=\left(m_{i j}\right)$ of operators we shall write

$$
M \in \Psi^{m}\left(\Sigma_{1}\right) \Longleftrightarrow \forall i, j, m_{i j} \in \Psi^{m}\left(\Sigma_{1}\right) .
$$

We use an analogous notation for a matrix of symbols. The matrix $\operatorname{Op}(M)$ is the matrix of operators $\left(\mathrm{Op}\left(m_{i j}\right)\right)$. Recall that $\phi^{\rho}$ is defined as a subspace of $C^{\infty}(\mathbb{R})$ by

$$
f \in S^{\rho} \Longleftrightarrow \forall \alpha \in \mathbb{N},\left|f^{(\alpha)}(x)\right| \leq C_{\alpha}\langle x\rangle^{\rho-\alpha} .
$$

We will study $U\left(0, t_{\epsilon}\right) F_{t_{\epsilon}}^{T}$ microlocally. We first observe that $F_{t_{\epsilon}}^{T}$ has high frequencies in $\xi$. We show that for $L_{0}>0$ we have (see Lemma 10.3):

$$
\begin{aligned}
\text { Op }\left(\chi\left(\frac{\langle\xi\rangle}{\langle q\rangle} \leq L_{0}\right)\right) F_{t_{\epsilon}}^{T} \rightarrow 0, \quad T \rightarrow \infty, \\
F_{t_{\epsilon}}^{T} \rightarrow 0, \quad T \rightarrow \infty .
\end{aligned}
$$

We then study the propagation of singularities of $\mathrm{e}^{-i t_{\epsilon} H}$. Because of $(10.2),(10.3)$ we are interested in the propagation of "outgoing" singularities located in

$$
\left\{(\widehat{r}, \omega ; \xi, q) ; \widehat{r} \geq-t_{\epsilon}-\left|\Theta\left(\mathrm{e}^{-\kappa_{+} T}\right)\right|,\langle\xi\rangle \geq L_{0}\langle q\rangle\right\} .
$$


We will show that these singularities stay away from the surface of the star. Because of (10.4) it follows (modulo a small error term):

$$
\left(1-\phi_{\delta}\right)(.-\widehat{z}(0, \theta)) \mathrm{e}^{-i t_{\epsilon} H} F_{t_{\epsilon}}^{T} \longrightarrow 0
$$

for an appropriate choice of $\delta>0$. Using (10.5) we show that

$$
\|\left(U\left(0, t_{\epsilon}\right)-\phi_{\delta}(.-\widehat{z}(0, \theta)) \mathrm{e}^{-i t_{\epsilon} H} F_{t_{\epsilon}}^{T} \|\right.
$$

is small for $t_{\epsilon}, T$ large.

This will prove Proposition 10.1.

\subsection{The geometric optics approximation and its properties}

We need the following lemmas:

LEMMA 10.1. - We have

$$
\left\|F_{t_{0}}^{T}\right\|_{L^{1}\left(\mathbb{R} ;\left(L^{2}\left(S^{2}\right)\right)^{4}\right)} \longrightarrow 0, \quad T \rightarrow \infty .
$$

Proof. - The lemma follows from the following calculation:

$$
\begin{aligned}
& \left\|F_{t_{0}}^{T}\right\|_{L^{1}\left(\mathbb{R} ;\left(L^{2}\left(S^{2}\right)\right)^{4}\right)} \\
& =\int_{-t_{0}-\left|\vartheta\left(\mathrm{e}^{-\kappa_{+} T}\right)\right|}^{-t_{0}} \frac{1}{\sqrt{-\kappa_{+}\left(\widehat{r}+t_{0}\right)}} \\
& \quad \times\left(\int_{S^{2}}\left(\left|\tilde{f}_{3}\right|^{2}+\left|\tilde{f}_{2}\right|^{2}\right)\left(T+\frac{1}{\kappa_{+}} \ln \left(-\left(\widehat{r}+t_{0}\right)\right)-\frac{1}{\kappa_{+}} \ln \widehat{A}(\theta), \omega\right) \mathrm{d} \omega\right)^{\frac{1}{2}} \mathrm{~d} \widehat{r} \\
& =\int_{I}-\sqrt{\kappa_{+}} \mathrm{e}^{\kappa_{+} \frac{1}{2}(y-T)}\left(\int_{S^{2}}\left(\left|\tilde{f}_{3}\right|^{2}+\left|\tilde{f}_{2}\right|^{2}\right)\left(y-\frac{1}{\kappa_{+}} \ln \widehat{A}(\theta), \omega\right) \mathrm{d} \omega\right)^{\frac{1}{2}} \mathrm{~d} y \rightarrow 0 .
\end{aligned}
$$

Here $I$ is a compact interval depending on the support of $\widetilde{f}$.

LEMMA 10.2. - We have

$$
\left\|U_{\leftarrow}\left(t_{0}, T\right) \tilde{f}-F_{t_{0}}^{T}\right\| \longrightarrow 0, \quad T \rightarrow \infty .
$$

Proof. - Let $u=U_{\leftarrow}\left(t_{0}, T\right) \tilde{f}$. Recall that for $T$ sufficiently large we have

$$
\begin{aligned}
& u_{1}(\widehat{r}, \omega)=\sqrt{\frac{1-\dot{\grave{z}}}{1+\dot{\widehat{z}}}}\left(\widehat{\tau}\left(\widehat{r}+t_{0}, \theta\right), \theta\right) \tilde{f}_{3}\left(\widehat{r}+t_{0}+T-2 \widehat{\tau}\left(\widehat{r}+t_{0}, \theta\right), \omega\right), \\
& u_{4}(\widehat{r}, \omega)=-\sqrt{\frac{1-\dot{\widehat{z}}}{1+\dot{\widehat{z}}}}\left(\widehat{\tau}\left(\widehat{r}+t_{0}, \theta\right), \theta\right) \tilde{f}_{2}\left(\widehat{r}+t_{0}+T-2 \widehat{\tau}\left(\widehat{r}+t_{0}, \theta\right), \omega\right), \\
& u_{2}=u_{3}=0 .
\end{aligned}
$$


We will also use

$$
\begin{gathered}
\widehat{\tau}\left(x_{0}, \theta\right)=-\frac{1}{2 \kappa_{+}} \ln \left(-x_{0}\right)+\frac{1}{2 \kappa_{+}} \ln \widehat{A}(\theta)+\theta\left(x_{0}\right), \quad x_{0} \rightarrow 0^{-}, \\
1+\dot{\widehat{z}}\left(\widehat{\tau}\left(x_{0}, \theta\right)\right)=-2 \kappa_{+} x_{0}+\Theta\left(x_{0}^{2}\right), \quad x_{0} \rightarrow 0^{-} .
\end{gathered}
$$

We have

$$
\hat{\ell}(\widehat{r}, \theta):=\sqrt{-\kappa_{+} \widehat{r}} \sqrt{\frac{1-\dot{\widehat{z}}}{1+\dot{\grave{z}}}}(\widehat{\tau}(\widehat{r}, \theta), \theta)=\sqrt{\frac{-2 \kappa_{+} \widehat{r}+\theta\left(\widehat{r}^{2}\right)}{-2 \kappa_{+} \widehat{r}+\theta\left(\widehat{r}^{2}\right)}}, \quad \widehat{r} \rightarrow 0^{-} .
$$

We calculate

$$
\begin{aligned}
& \left\|\left(U_{\leftarrow}\left(t_{0}, T\right) \tilde{f}-F_{t_{0}}^{T}\right)_{1}\right\|^{2}=\int_{S^{2}} \int_{\mathbb{R}}\left|\left(u_{1}-F_{1}^{T}\right)\right|^{2} \mathrm{~d} \widehat{r} \mathrm{~d} \omega \\
& =\int_{S^{2}} \int_{I}\left|\hat{\ell}\left(-\widehat{A}(\theta) \mathrm{e}^{\kappa_{+}(y-T)}\right) \widetilde{f}_{3}\left(y+\theta\left(-\widehat{A}(\theta) \mathrm{e}^{\kappa_{+}(y-T)}\right), \omega\right)-\widetilde{f}_{3}(y, \omega)\right|^{2} \mathrm{~d} y \mathrm{~d} \omega \\
& \quad \longrightarrow 0, \quad T \rightarrow \infty .
\end{aligned}
$$

Here $I$ is a compact interval depending on the support of $\widetilde{f}$.

Let $G^{T}=\mathrm{e}^{-i t_{0} H} F_{t_{0}}^{T}$.

Corollary 10.1. - We have

$$
F_{t_{0}}^{T} \rightarrow 0, \quad G^{T} \rightarrow 0, \quad U\left(t_{0}, T\right) \tilde{f} \rightarrow 0 .
$$

Lemma 10.3. - Let $\chi \in \phi^{-\rho}$ and $\rho>0$. Then we have for all $M>0$ and uniformly in $t_{\epsilon}$ :

$$
\begin{aligned}
& \mathrm{Op}\left(\chi\left(\frac{\langle\xi\rangle}{\langle q\rangle}\right)\langle q\rangle^{M}\right) F_{t_{\epsilon}, n}^{T} \longrightarrow 0, \quad T \rightarrow \infty \widehat{\mathcal{H}}, \\
& \mathrm{Op}\left(\chi\left(\langle(\xi, q)\rangle\langle q\rangle^{M}\right) F_{t_{\epsilon}, n}^{T} \longrightarrow 0, \quad T \rightarrow \infty \widehat{\mathcal{H}} .\right.
\end{aligned}
$$

Proof. - We only show (10.6), the proof of (10.7) being analogous. Let us write

$$
\begin{aligned}
F_{0, n}^{T} & =K_{T} \widehat{g}, \\
\widehat{g}(\widehat{r}, \theta) & =\frac{1}{\sqrt{-\kappa_{+} \widehat{r}}}\left(\widetilde{f}_{3}, 0,0,-\widetilde{f}_{2}\right)\left(\frac{1}{\kappa_{+}} \ln (-\widehat{r})-\frac{1}{\kappa_{+}} \ln \widehat{A}(\theta), \theta\right), \\
\left(K_{T} \widehat{g}\right)(\widehat{r}, \theta) & =\mathrm{e}^{\frac{1}{2} \kappa_{+} T \widehat{g}}\left(\left(\widehat{r}+t_{\epsilon}\right) \mathrm{e}^{\kappa_{+} T}, \theta\right)
\end{aligned}
$$

and we shall also consider $K_{T}$ as an operator on $\widehat{\mathscr{H}}^{\ell}$ (rather than on $\widehat{\mathscr{H}}$ ). Let us write

$$
\widehat{g}=\sum_{\ell} \widehat{g}^{\ell}, \quad \hat{g}^{\ell} \in \widehat{\mathscr{H}}^{\ell} \text { for all } \ell .
$$

Thus

$$
\chi\left(\frac{\left\langle D_{\widehat{r}}\right\rangle}{\left\langle D_{\theta}\right\rangle}\right)\left\langle D_{\theta}\right\rangle^{M} K_{T} \widehat{g}=\sum_{\ell} \chi\left(\frac{\left\langle D_{\widehat{r}}\right\rangle}{\langle\ell\rangle}\right)\langle\ell\rangle^{M} K_{T} \widehat{g}^{\ell}
$$


We have

$$
\mathcal{F}\left(K_{T} \widehat{g}^{\ell}\right)(\xi)=\mathcal{F}\left(\widehat{g}^{\ell}\right)\left(\xi \mathrm{e}^{-\kappa_{+} T}\right) \mathrm{e}^{-\frac{1}{2} \kappa_{+} T} \mathrm{e}^{i t_{\epsilon} \xi},
$$

where $\mathcal{F}$ denotes the Fourier transform in $\widehat{r}$. Note that

$$
\begin{gathered}
\left\|\chi\left(\frac{\left\langle D_{\widehat{r}}\right\rangle}{\left\langle D_{\theta}\right\rangle}\right)\left\langle D_{\theta}\right\rangle^{M} K_{T} \widehat{g}\right\|^{2}=\sum_{\ell}\left\|\chi\left(\frac{\langle\xi\rangle}{\langle\ell\rangle}\right)\langle\ell\rangle^{M} \mathcal{F}\left(\widehat{g}^{\ell}\right)\left(\xi \mathrm{e}^{-\kappa_{+} T}\right) \mathrm{e}^{-\frac{1}{2}\left(\kappa_{+} T\right)}\right\|^{2}, \\
\forall T, \quad\left\|\chi\left(\frac{\langle\xi\rangle}{\langle\ell\rangle}\right)\langle\ell\rangle^{M} \mathcal{F}\left(\widehat{g}^{\ell}\right)\left(\xi \mathrm{e}^{-\kappa_{+} T}\right) \mathrm{e}^{-\frac{1}{2} \kappa_{+} T}\right\|^{2} \leq\langle\ell\rangle^{2 M}\left\|\widehat{g}^{\ell}\right\|^{2}, \quad \sum_{\ell}\langle\ell\rangle^{2 M}\left\|\widehat{g}^{\ell}\right\|^{2}<\infty .
\end{gathered}
$$

It is therefore sufficient to show

$$
\forall \ell, \quad\left\|\chi\left(\frac{\langle\xi\rangle}{\langle l\rangle}\right)\langle\ell\rangle^{M} \mathcal{F}\left(\widehat{g}^{\ell}\right)\left(\xi \mathrm{e}^{-\kappa_{+} T}\right) \mathrm{e}^{-\frac{1}{2}\left(\kappa_{+} T\right)}\right\| \longrightarrow 0, \quad T \rightarrow \infty .
$$

But, by the Lebesgue Theorem,

$$
\left\|\chi\left(\frac{\langle\xi\rangle}{\langle\ell\rangle}\right)\langle\ell\rangle^{M} \mathcal{F}\left(\widehat{g}^{\ell}\right)\left(\xi \mathrm{e}^{-\kappa_{+} T}\right) \mathrm{e}^{-\frac{1}{2} \kappa_{+} T}\right\|^{2}=\int\left|\chi\left(\frac{\left\langle\xi \mathrm{e}^{\kappa_{+} T}\right\rangle}{\langle\ell\rangle}\right)\langle\ell\rangle^{M} \mathcal{F}\left(\widehat{g}^{\ell}\right)(\xi)\right|^{2} \mathrm{~d} \xi \longrightarrow 0 .
$$

This proves (10.6).

\subsection{Diagonalization}

Let $\nu_{1}>0, \nu_{2}>0, j_{ \pm}, \chi \in C^{\infty}(\mathbb{R}), \operatorname{supp} \chi \subset \mathbb{R} \backslash\left[-\nu_{2}, \nu_{2}\right], \chi \equiv 1$ on $\mathbb{R} \backslash\left[-2 \nu_{2}, 2 \nu_{2}\right]$,

$$
j_{+}(x)=\left\{\begin{array}{ll}
1 & x \geq 2 \nu_{1}, \\
0 & x \leq \nu_{1},
\end{array} \quad j_{-}(x)=\left\{\begin{array}{ll}
1 & x \leq-2 \nu_{1}, \\
0 & x \geq-\nu_{1},
\end{array} \quad j^{2}(x)=j_{-}^{2}(x)+j_{+}^{2}(x) .\right.\right.
$$

We put

$$
\begin{aligned}
& \widetilde{W}_{ \pm}(x)=\tilde{c}_{ \pm}\left(\begin{array}{cc}
a_{0}\left(x \pm \ell^{\prime}\right) & \pm k^{\prime}-\sqrt{k^{\prime 2}+a_{0}^{2}\left(x \pm \ell^{\prime}\right)^{2}} \\
\sqrt{k^{\prime 2}+a_{0}^{2}\left(x \pm \ell^{\prime}\right)^{2}} \mp k^{\prime} & a_{0}\left(x \pm \ell^{\prime}\right)
\end{array}\right) \\
& \tilde{c}_{ \pm}=\frac{e_{ \pm}\left(x+\ell^{\prime}\right)}{\sqrt{2} \sqrt[4]{k^{\prime 2}+a_{0}^{2}\left(x \pm \ell^{\prime}\right)^{2}} \sqrt{\sqrt{k^{\prime 2}+a_{0}^{2}\left(x \pm \ell^{\prime}\right)^{2}} \mp k^{\prime}}}, \\
& e_{+}(x)=\operatorname{sign} x, \quad e_{-}(x)=1 \text {, } \\
& W_{ \pm}^{0}=\widetilde{W}_{ \pm}\left(\frac{q}{|\xi|}\right) j_{ \pm}\left(\frac{\xi}{|q|}\right) \chi\left(h^{2} \sqrt{k^{\prime 2} \xi^{2}+a_{0}^{2}\left(\ell^{\prime} \xi+q\right)^{2}}\right) \\
& W_{ \pm}=u\left(\begin{array}{cc}
W_{ \pm}^{0} & 0 \\
0 & W_{ \pm}^{0}
\end{array}\right) \\
& \text { (10.9) } W(\widehat{r}, \theta, \xi, q)=W_{+}(\widehat{r}, \theta, \xi, q)+W_{-}(\widehat{r}, \theta, \xi, q) \text {. }
\end{aligned}
$$


By $(10.8) W(\widehat{r}, \theta, \xi, q)$ is only defined for $q \neq 0$ and $\xi \neq 0$. We note that for $\epsilon_{q}>0$ small enough and $|q|<\epsilon_{q}$ we have

$$
\begin{aligned}
W(\widehat{r}, \theta, \xi, q)=\mathcal{U}\left[\left(\begin{array}{cc}
\widetilde{W}_{+}(q /|\xi|) & 0 \\
0 & \widetilde{W}_{+}(q /|\xi|)
\end{array}\right)\right. & \left.+\left(\begin{array}{ll}
\widetilde{W}_{-}(q /|\xi|) & 0 \\
0 & \widetilde{W}_{-}(q /|\xi|)
\end{array}\right)\right] \\
& \times \chi\left(h^{2} \sqrt{k^{2} \xi^{2}+a_{0}^{2}\left(\ell^{\prime} \xi+q\right)^{2}}\right) .
\end{aligned}
$$

Indeed if $|q|<\epsilon_{q}$ we have on $\operatorname{supp} \chi\left(h^{2} \sqrt{k^{\prime 2} \xi^{2}+a_{0}^{2}\left(\ell^{\prime} \xi+q\right)^{2}}\right)$

$$
C\left(|\xi|^{2}+|q|^{2}\right) \geq \nu_{2}^{2} \Longrightarrow|\xi|^{2} \geq \frac{1}{C} \nu_{2}^{2}-\epsilon_{q}^{2} .
$$

But if $|\xi|^{2} \geq 4 \nu_{1}^{2}|q|^{2}$ we have $j_{ \pm}(\xi /|q|)=1$. Using (10.11) we see that this is fulfilled if $\epsilon_{q}$ is small enough. Therefore we define $W(\widehat{r}, \theta, \xi, q)$ for $q=0$ by (10.10). Similarly there exists $\epsilon_{\xi}>0$ such that if $|\xi|<\epsilon_{\xi}$ then

$$
W(\widehat{r}, \theta, \xi, q)=0 .
$$

Indeed on $\operatorname{supp} j_{ \pm}(\xi /|q|)$ we have $|q| \leq|\xi| / \nu_{1}$. If $|\xi| \leq \epsilon_{\xi}$, then

$$
h^{2} \sqrt{k^{2} \xi^{2}+a_{0}^{2}\left(\ell^{\prime} \xi+q\right)^{2}} \leq C \epsilon_{\xi}<\nu_{2}
$$

for $\epsilon_{\xi}$ small enough and thus $\chi\left(h^{2} \sqrt{k^{\prime 2} \xi^{2}+a_{0}^{2}\left(\ell^{\prime} \xi+q\right)^{2}}\right)=0$. Therefore we define $W(\widehat{r}, \theta, 0, q)=0 . W(\widehat{r}, \theta, \xi, q)$ with these definitions is a matrix of smooth functions. We want to check that $W(\widehat{r}, \theta ; \xi, q) \in S^{0}\left(\Sigma_{1}\right)$. To this purpose we apply the symplectic change of coordinates

$$
r_{*}=r_{*}(\widehat{r}, \theta), \quad \theta^{*}=\theta, \quad q^{*}=\ell^{\prime} \xi+q, \quad \xi^{*}=k^{\prime} \xi .
$$

Under this change of coordinates we obtain the symbol

$$
\begin{aligned}
& \widehat{W}\left(r_{*}, \theta^{*}, \xi^{*}, q^{*}\right)= \mathcal{U}\left[\begin{array}{cc}
\widehat{W}_{+}\left(q^{*} /\left|\xi^{*}\right|\right) & 0 \\
0 & \widehat{W}_{+}\left(q^{*} /\left|\xi^{*}\right|\right)
\end{array}\right) j_{+}\left(\xi^{*} /\left|k^{\prime} q^{*}-\ell^{\prime} \xi^{*}\right|\right) \\
&\left.+\left(\begin{array}{cc}
\widehat{W}_{-}\left(q^{*} /\left|\xi^{*}\right|\right) & 0 \\
0 & \widehat{W}_{-}\left(q^{*} /\left|\xi^{*}\right|\right)
\end{array}\right) j_{-}\left(\xi^{*} /\left|k^{\prime} q^{*}-\ell^{\prime} \xi^{*}\right|\right)\right] \\
& \times \chi\left(h^{2} \sqrt{\left|\xi^{*}\right|^{2}+a_{0}^{2}\left|q^{*}\right|^{2}}\right) \\
& \widehat{W}_{ \pm}(x)=\hat{c}_{ \pm}\left(\begin{array}{c}
a_{0} x \\
\sqrt{1+a_{0}^{2} x^{2}} \mp 1-\sqrt{1+a_{0}^{2} x^{2}} \\
e_{ \pm}(x)
\end{array}\right), \\
& \hat{c}_{ \pm}=\frac{a_{0} x}{\sqrt{2} \sqrt[4]{1+a_{0}^{2} x^{2}} \sqrt{\sqrt{1+a_{0}^{2} x^{2}} \mp 1}}
\end{aligned}
$$

It is sufficient to check

$$
\widehat{W}\left(r_{*}, \theta^{*}, \xi^{*}, q^{*}\right) \in S^{0} .
$$


On supp $j_{ \pm}\left(\xi^{*} /\left|k^{\prime} q^{*}-\ell^{\prime} \xi^{*}\right|\right),\left|q^{*}\right| /\left|\xi^{*}\right|$ remains bounded. The functions

$$
f^{ \pm}(x)=\frac{|x|}{\sqrt{2} \sqrt[4]{x^{2}+1} \sqrt{\sqrt{x^{2}+1} \mp 1}}, \quad g^{ \pm}= \pm \frac{\sqrt{\sqrt{x^{2}+1} \mp 1}}{\sqrt{2} \sqrt[4]{x^{2}+1}}
$$

are $C^{\infty}(\mathbb{R})$ functions with

$$
\left|\partial^{\alpha} f^{ \pm}(x)\right| \leq C_{\alpha}, \quad\left|\partial^{\alpha} g^{ \pm}(x)\right| \leq C_{\alpha} .
$$

In order to see that the estimate holds for $f^{+}$we note that $\check{f}(x)=\left(\sqrt{\sqrt{x^{2}+1}-1}\right) /|x|$ can be extended to an analytic function in a neighborhood of zero with $\check{f}(0)=1 / \sqrt{2}$. We obtain

$$
\left|\partial_{q^{*}} \widehat{W}_{ \pm}\left(\frac{q^{*}}{\xi^{*}}\right)\right| \leq C \frac{1}{\left|\xi^{*}\right|}, \quad\left|\partial_{\xi^{*}} \widehat{W}_{ \pm}\left(\frac{q^{*}}{\xi^{*}}\right)\right| \leq C \frac{\left|q^{*}\right|}{\left|\xi^{*}\right|^{2}} .
$$

But on $\operatorname{supp} j_{ \pm}\left(\xi^{*} /\left|k^{\prime} q^{*}-\ell^{\prime} \xi^{*}\right|\right) \cap \operatorname{supp} \chi\left(h^{2} \sqrt{\left|\xi^{*}\right|^{2}+a_{0}^{2}\left|q^{*}\right|^{2}}\right)$ we have

$$
\frac{1}{\left|\xi^{*}\right|} \lesssim \frac{1}{\left\langle\xi^{*}\right\rangle}, \quad \frac{1}{\left|\xi^{*}\right|} \lesssim \frac{1}{\left\langle q^{*}\right\rangle}
$$

We now have to estimate derivatives on

$$
j_{ \pm}\left(\frac{\xi^{*}}{\left|k^{\prime} q^{*}-\ell^{\prime} \xi^{*}\right|}\right) \chi\left(h^{2} \sqrt{\left|\xi^{*}\right|^{2}+a_{0}^{2}\left|q^{*}\right|^{2}}\right) .
$$

In the region $k^{\prime} q^{*}-\ell^{\prime} \xi^{*}>0$ we find

$$
\begin{aligned}
& \left|\partial_{\xi^{*}} j_{ \pm}\left(\frac{\xi^{*}}{\left|k^{\prime} q^{*}-\ell^{\prime} \xi^{*}\right|}\right)\right| \\
& =\left|j_{ \pm}^{\prime}\left(\frac{\xi^{*}}{\left|k^{\prime} q^{*}-\ell^{\prime} \xi^{*}\right|}\right)\left(\frac{1}{k^{\prime} q^{*}-\ell^{\prime} \xi^{*}}+\frac{\xi^{*} \ell^{\prime}}{\left(k^{\prime} q^{*}-\ell^{\prime} \xi^{*}\right)^{2}}\right)\right| \lesssim \frac{1}{\left|\xi^{*}\right|}, \\
& \left|\partial_{q^{*}} j_{ \pm}^{\prime}\left(\frac{\xi^{*}}{\left|k^{\prime} q^{*}-\ell^{\prime} \xi^{*}\right|}\right)\right| \lesssim \frac{1}{\left|\xi^{*}\right|} .
\end{aligned}
$$

We then use (10.15). Derivatives in $r_{*}, \theta^{*}$, derivatives on $\chi\left(h^{2} \sqrt{\left|\xi^{*}\right|^{2}+a_{0}^{2}\left|q^{*}\right|^{2}}\right), \mathcal{U}$ as well as higher order derivatives can be controlled in a similar way. Let $\widetilde{\chi} \in C^{\infty}(\mathbb{R})$, $\operatorname{supp} \tilde{\chi} \subset \mathbb{R} \backslash\left[-\frac{1}{2} \nu_{2}, \frac{1}{2} \nu_{2}\right]$ with $\tilde{\chi} \chi=\chi$. We next put

$$
\begin{gathered}
\lambda=h^{2} \sqrt{k^{\prime 2} \xi^{2}+a_{0}^{2}\left(\ell^{\prime} \xi+q\right)^{2}} \tilde{\chi}\left(h^{2} \sqrt{k^{\prime 2} \xi^{2}+a_{0}^{2}\left(\ell^{\prime} \xi+q\right)^{2}}\right), \\
\check{H}_{d}=\lambda \Gamma^{1}, \quad \widetilde{H}_{d}=\operatorname{Op}\left(\check{H}_{d}\right) .
\end{gathered}
$$

We have

$$
H \mathrm{Op}\left(W_{ \pm}\right)=\mathrm{Op}\left(W_{ \pm}\right) \widetilde{H}_{d}+\mathrm{Op}\left(R_{ \pm}\right)+\mathrm{Op}\left(\widetilde{R}_{ \pm}\right)
$$

with

$$
\begin{gathered}
R_{ \pm}=R_{ \pm}^{1}+R_{ \pm}^{2}, R_{ \pm}^{k}=\left(r_{i j}^{k \pm}\right) \\
\operatorname{supp} r_{i j}^{1 \pm} \subset \operatorname{supp} j_{ \pm}(\xi /|q|) \cap \operatorname{supp} \chi\left(h^{2} \sqrt{k^{\prime 2} \xi^{2}+a_{0}^{2}\left|\ell^{\prime} \xi+q\right|^{2}}\right)
\end{gathered}
$$




$$
\begin{gathered}
r_{i j}^{1 \pm} \in S^{0}\left(\Sigma_{1}\right), \quad r_{i j}^{2 \pm} \in S^{-\infty}\left(\Sigma_{1}\right), \\
\left(\begin{array}{cc}
r_{13}^{k \pm} & r_{14}^{k \pm} \\
r_{23}^{k \pm} & r_{24}^{k \pm}
\end{array}\right)=\left(\begin{array}{cc}
r_{31}^{k \pm} & r_{32}^{k \pm} \\
r_{41}^{k \pm} & r_{42}^{k \pm}
\end{array}\right)=0, \\
\widetilde{R}_{ \pm}=\widetilde{R}_{ \pm}^{1}+\widetilde{R}_{ \pm}^{2}, \quad \widetilde{R}_{ \pm}^{2} \in \Psi^{-1}\left(\Sigma_{1}\right), \\
\widetilde{R}_{ \pm}^{1}=U p \Gamma^{4}\left(\begin{array}{cc}
W_{ \pm}^{0} & 0 \\
0 & W_{ \pm}^{0}
\end{array}\right), \\
p(\widehat{r}, \theta)=\frac{m \sqrt{\Delta}}{\sigma}\left(\rho-\sqrt{r^{2}+a^{2}}\right)+b_{0} .
\end{gathered}
$$

We need a better estimate on the remainder.

LEMmA 10.4. - There exists $M=\left(m_{i j}\right)$ such that for all $j, m_{j j}=1$ and for all $i \neq j, m_{i j} \in \Psi^{-1}\left(\Sigma_{1}\right)$ as well as $r_{j} \in S^{0}\left(\Sigma_{1}\right), j=1, \ldots, 4$ such that for

$$
H_{d}=\widetilde{H}_{d}+\operatorname{Op}\left(\operatorname{Diag}\left(r_{1}, r_{2}, r_{3}, r_{4}\right)\right)
$$

we have:

$$
H \mathrm{Op}(W) M-\mathrm{Op}(W) M H_{d} \in \Psi^{-1}\left(\Sigma_{1}\right)
$$

Proof. - We can construct independently $M_{ \pm}$and $r_{j}^{ \pm}$such that (10.24) is fulfilled for $M$ replaced by $M_{ \pm}, r_{j}$ by $r_{j}^{ \pm}$and $W$ by $W_{ \pm}$. We then put $M=\frac{1}{2}\left(M_{+}+M_{-}\right)$, $r_{j}=\frac{1}{2}\left(r_{j}^{+}+r_{j}^{-}\right)$. We only consider the + case and drop the index + . We are looking for $M$ in the form

$$
\begin{gathered}
M=\left(\begin{array}{cc}
A & B \\
C & D
\end{array}\right), \quad B, C \in \Psi^{-1}\left(\Sigma_{1}\right), \\
A=\left(\begin{array}{cc}
1 & \mathrm{Op}\left(\alpha_{1}\right) \\
\mathrm{Op}\left(\alpha_{2}\right) & 1
\end{array}\right), \quad D=\left(\begin{array}{ll}
1 & \mathrm{Op}\left(\delta_{1}\right) \\
\mathrm{Op}\left(\delta_{2}\right) & 1
\end{array}\right), \\
\alpha_{j}, \delta_{j} \in S^{-1}\left(\Sigma_{1}\right), \quad j=1,2 .
\end{gathered}
$$

If $M$ is of this form it is sufficient that

$$
\begin{aligned}
& \mathrm{Op}(R)+\mathrm{Op}(\widetilde{R})-\mathrm{Op}(W)\left[M, \widetilde{H}_{d}\right]=\mathrm{Op}(W) \mathrm{Op}(\left.\operatorname{Diag}\left(r_{1}, \ldots, r_{4}\right)\right) \\
&+\widehat{R}, \quad \widehat{R} \in \Psi^{-1}\left(\Sigma_{1}\right) .
\end{aligned}
$$

Here we have used that

$$
M=I_{d}+R_{1}, \quad R_{1} \in \Psi^{-1}\left(\Sigma_{1}\right)
$$

Therefore

$$
M \operatorname{Op}\left(\operatorname{Diag}\left(r_{1}, \ldots, r_{4}\right)\right)=\operatorname{Op}\left(\operatorname{Diag}\left(r_{1}, \ldots, r_{4}\right)\right)+R_{2}, \quad R_{2} \in \Psi^{-1}\left(\Sigma_{1}\right) .
$$


Recalling that $\widetilde{H}_{d}=\Lambda \operatorname{Diag}\left(1_{d},-1_{d}\right)$ and $\Lambda:=\operatorname{Op}(\lambda) \operatorname{Diag}(1,-1)$ we find

$$
\left[M, \widetilde{H}_{d}\right]=\left(\begin{array}{cc}
{[A, \Lambda]} & -\{B, \Lambda\} \\
\{C, \Lambda\} & -[D, \Lambda]
\end{array}\right) .
$$

If $\mathcal{U}^{*} R^{1}=\left(\begin{array}{cc}R_{11} & 0 \\ 0 & R_{22}\end{array}\right)$ and $\mathcal{U}^{*} \widetilde{R}^{1}=\left(\begin{array}{cc}0 & R_{12} \\ R_{21} & 0\end{array}\right)$ we have to find $A, B, C, D, r_{j}$ such that

$$
\begin{aligned}
& -W^{0}[A, \Lambda]+R_{11}=W^{0} \operatorname{Diag}\left(r_{1}, r_{2}\right)+\widehat{R}_{11}, \quad \widehat{R}_{11} \in \Psi^{-1}\left(\Sigma_{1}\right), \\
& W^{0}[D, \Lambda]+R_{22}=W^{0} \operatorname{Diag}\left(r_{3}, r_{4}\right)+\widehat{R}_{22}, \quad \widehat{R}_{22} \in \Psi^{-1}\left(\Sigma_{1}\right), \\
& W^{0}\{B, \Lambda\}+R_{12} \in \Psi^{-1}\left(\Sigma_{1}\right), \\
& -W^{0}\{C, \Lambda\}+R_{21} \in \Psi^{-1}\left(\Sigma_{1}\right) .
\end{aligned}
$$

We consider equations (10.27), (10.30). On

$$
\operatorname{supp} j_{+}(\xi /|q|) \cap \operatorname{supp} \chi\left(h^{2} \sqrt{k^{\prime 2} \xi^{2}+a_{0}^{2}\left(\ell^{\prime} \xi+q\right)^{2}}\right)
$$

the matrix $W^{0}$ is invertible. Let

$$
\left(W^{0}\right)^{-1} R_{11}=\left(\begin{array}{ll}
\check{r}_{11} & \check{r}_{12} \\
\check{r}_{21} & \check{r}_{22}
\end{array}\right) .
$$

As

$$
[A, \Lambda]=\left(\begin{array}{cc}
0 & -2 \mathrm{Op}\left(\alpha_{1} \lambda\right) \\
2 \mathrm{Op}\left(\alpha_{2} \lambda\right) & 0
\end{array}\right)+R_{3}, \quad R_{3} \in S^{-1}\left(\Sigma_{1}\right)
$$

we have to solve on $\operatorname{supp}\left(j_{+}(\xi /|q|)\right) \cap \operatorname{supp}\left(\chi\left(h^{2} \sqrt{k^{\prime 2} \xi^{2}+a_{0}^{2}\left(\ell^{\prime} \xi+q\right)^{2}}\right)\right)$ :

$$
\begin{aligned}
2 \alpha_{1} \lambda+\check{r}_{12} & \in S^{-1}\left(\Sigma_{1}\right), \\
-2 \alpha_{2} \lambda+\check{r}_{21} & \in S^{-1}\left(\Sigma_{1}\right),
\end{aligned}
$$

which can be achieved by

$$
\alpha_{1}=-\frac{\check{r}_{12}}{2 \lambda}, \quad \alpha_{2}=\frac{\check{r}_{21}}{2 \lambda} .
$$

In order to solve (10.30) we have to use the special structure of $R_{21}$. Indeed we have $R_{21}=i p W^{0}$. We try

$$
C=i\left(\begin{array}{cc}
\mathrm{Op}\left(\gamma_{1}\right) & 0 \\
0 & \mathrm{Op}\left(\gamma_{2}\right)
\end{array}\right), \quad \gamma_{1}, \gamma_{2} \in S^{-1}\left(\Sigma_{1}\right)
$$

Then

$$
-W^{0}\{C, \Lambda\}+R_{21}=i W^{0}\left(\begin{array}{cc}
-2 \mathrm{Op}\left(\gamma_{1} \lambda\right) & 0 \\
0 & 2 \mathrm{Op}\left(\gamma_{2} \lambda\right)
\end{array}\right)+i W^{0} p
$$


and therefore we can take on $\operatorname{supp} j_{+}(\xi /|q|) \cap \operatorname{supp} \chi\left(h^{2} \sqrt{k^{\prime 2} \xi^{2}+a_{0}^{2}\left(\ell^{\prime} \xi+q\right)^{2}}\right)$ :

$$
\gamma_{1}=-\gamma_{2}=\frac{p}{2 \lambda}
$$

This concludes the proof of the lemma.

LEMMA 10.5. - We have for all $t_{\epsilon}>0$ and all $s$ such that $0 \leq s \leq t_{\epsilon}$,

$$
\left(\mathrm{e}^{-i s H}-\mathrm{Op}(W) M \mathrm{e}^{-i s H_{d}} M^{-1} \mathrm{Op}\left(W^{*}\right)\right) F_{t_{\epsilon}}^{T} \longrightarrow 0, \quad T \rightarrow \infty .
$$

Proof. - We have

$$
\begin{aligned}
& \left(\mathrm{e}^{-i s H}-\mathrm{Op}(W) M \mathrm{e}^{-i s H_{d}} M^{-1} \mathrm{Op}\left(W^{*}\right)\right) F_{t_{\epsilon}}^{T} \\
& \quad=\int_{0}^{s} \mathrm{e}^{-i \tau H}\left(H \mathrm{Op}(W) M-\mathrm{Op}(W) M H_{d}\right) \mathrm{e}^{-i(s-\tau) H_{d}} M^{-1} \mathrm{Op}\left(W^{*}\right) F_{t_{\epsilon}}^{T} \mathrm{~d} \tau \\
& \quad+\mathrm{e}^{-i s H}\left(1-\mathrm{Op}(W) \mathrm{Op}\left(W^{*}\right)\right) F_{t_{\epsilon}}^{T} \\
& =: I_{1}(s, T)+I_{2}(s, T) .
\end{aligned}
$$

By Lemma 10.3 we have

$$
I_{2}(s, T) \longrightarrow 0, \quad T \rightarrow \infty .
$$

Using Lemmas 10.4 and 10.3 we see that the first term can be estimated by

$$
\left\|I_{1}(s, T)\right\| \lesssim\left\|\left\langle H_{d}\right\rangle^{-1} M^{-1} \mathrm{Op}\left(W^{*}\right) F_{t_{\epsilon}}^{T}\right\| \longrightarrow 0, \quad T \rightarrow \infty .
$$

Let $\widetilde{N}_{ \pm}=\widetilde{W}_{ \pm}(0)$ and

$$
N=\mathcal{U}\left(\left(\begin{array}{ll}
\tilde{N}_{+} & 0 \\
0 & \tilde{N}_{+}
\end{array}\right) j_{+}\left(\frac{\xi}{|q|}\right)+\left(\begin{array}{ll}
\tilde{N}_{-} & 0 \\
0 & \tilde{N}_{-}
\end{array}\right) j_{-}\left(\frac{\xi}{|q|}\right)\right) \chi\left(h^{2} \sqrt{k^{\prime 2} \xi^{2}+a_{0}^{2}\left(\ell^{\prime} \xi+q\right)^{2}}\right) .
$$

We have

$$
\begin{array}{r}
\tilde{N}_{+}=\frac{1}{\sqrt{2}}\left(\begin{array}{cc}
\sqrt{1+\alpha} & -\sqrt{1-\alpha} \operatorname{sign}(\cos \theta) \\
\sqrt{1-\alpha} \operatorname{sign}(\cos \theta) & \sqrt{1+\alpha}
\end{array}\right) j_{+}\left(\frac{\xi}{|q|}\right) \\
\times \chi\left(h^{2} \sqrt{k^{\prime 2} \xi^{2}+a_{0}^{2}\left(\ell^{\prime} \xi+q\right)^{2}}\right), \\
\tilde{N}_{-}=\frac{1}{\sqrt{2}}\left(\begin{array}{cc}
-\operatorname{sign}(\cos \theta) \sqrt{1-\alpha} & -\sqrt{1+\alpha} \\
\sqrt{1+\alpha} & -\operatorname{sign}(\cos \theta) \sqrt{1-\alpha}
\end{array}\right) j_{-}\left(\frac{\xi}{|q|}\right) \\
\times \chi\left(h^{2} \sqrt{k^{\prime 2} \xi^{2}+a_{0}^{2}\left(\ell^{\prime} \xi+q\right)^{2}}\right) .
\end{array}
$$

with $\alpha$ as in Chapter 4 . Note that the matrices $\tilde{N}_{ \pm}$are smooth $\left(\alpha\left(\widehat{r}, \frac{1}{2} \pi\right)=1\right)$. From the lipschitz continuity of $f^{ \pm}, g^{ \pm}$we infer

$$
(W-N) \frac{\langle\xi\rangle}{\langle q\rangle} \in S^{0}\left(\Sigma_{1}\right) \text {. }
$$


Therefore by Lemma 10.3

$$
\left(\mathrm{Op}\left(W^{*}\right)-\mathrm{Op}\left(N^{*}\right)\right) F_{t_{\epsilon}}^{T} \longrightarrow 0 .
$$

Let us put

$$
\begin{aligned}
N_{\leftarrow} & =\left(j_{+}\left(\frac{\xi}{|q|}\right)+\left(\begin{array}{rrrr}
0 & -1 & 0 & 0 \\
1 & 0 & 0 & 0 \\
0 & 0 & 0 & -1 \\
0 & 0 & 1 & 0
\end{array}\right) j_{-}\left(\frac{\xi}{|q|}\right)\right) \chi\left(h^{2} \sqrt{k^{\prime 2} \xi^{2}+a_{0}^{2}\left(\ell^{\prime} \xi+q\right)^{2}}\right) \\
& =\left(j_{+}\left(\frac{\xi}{|q|}\right)+N_{\leftarrow}^{-} j_{-}\left(\frac{\xi}{|q|}\right)\right) \chi\left(h^{2} \sqrt{k^{\prime 2} \xi^{2}+a_{0}^{2}\left(\ell^{\prime} \xi+q\right)^{2}}\right) .
\end{aligned}
$$

Note that we have uniformly in $T$ large

$$
\mathrm{Op}\left(N^{*}\right) F_{t_{\epsilon}}^{T}=\mathrm{Op}\left(N_{\leftarrow}^{*}\right) F_{t_{\epsilon}}^{T}+\vartheta_{N, R}\left(\mathrm{e}^{-\kappa_{+} t_{\epsilon}}\right) .
$$

Here we have used that the commutators

$$
\left[\sqrt{1-\alpha}, \mathrm{Op}\left(j_{+}\left(\frac{\xi}{|q|}\right) \chi\left(h^{2} \sqrt{k^{\prime 2} \xi^{2}+a_{0}^{2} q^{2}}\right)\right)\right],
$$

etc. are all in $\Psi^{-1}\left(\Sigma_{1}\right)$.

\subsection{Study of the hamiltonian flow}

In this section we study the hamiltonian flow of

$$
P=h^{2} \sqrt{k^{2} \xi^{2}+a_{0}^{2}\left(\ell^{\prime} \xi+q\right)^{2}} .
$$

We denote $\phi_{t}$ the hamiltonian flow of $P$. Let for $L>0$

$$
\begin{aligned}
& \mathcal{E}_{L}:=\{(\widehat{r}, \theta ; \xi, q) ; \xi /|q| \geq L\}, \\
& \mathcal{J}_{L}^{t_{0}}:=\left\{(\widehat{r}, \theta ; \xi, q) ; \widehat{r} \geq-t_{0}-L^{-1}\right\} .
\end{aligned}
$$

Lemma 10.6. - For $t_{0}>0$ sufficiently large there exist $\delta>0$ and $L_{0}>0$ such that for all $L \geq L_{0}$ we have

$$
\forall 0 \leq s \leq t_{0}, \quad \phi_{s}\left(\mathcal{J}_{L}^{t_{0}} \cap \mathcal{E}_{L}\right) \subset\left\{(\widehat{r}, \theta ; \xi, q) ; \widehat{r} \geq \widehat{z}\left(t_{0}-s, \theta\right)+\delta\right\} .
$$

Proof. - We use the coordinates $\left(r_{*}, \theta^{*}, \xi^{*}, q^{*}\right)$ given by (10.13) and drop the star for $\theta: \theta=\theta^{*}$. Under this change of coordinates the hamiltonian becomes

$$
P^{*}=h^{2} \sqrt{\left|\xi^{*}\right|^{2}+a_{0}^{2}\left|q^{*}\right|^{2}}=E=\text { Const. }
$$


The hamiltonian equations are:

$$
\begin{gathered}
\dot{r}_{*}=\frac{h^{4} \xi^{*}}{E}, \\
\dot{\xi}^{*}=-\partial_{r_{*}} P^{*}, \\
\dot{\theta}=\frac{h^{4} a_{0}^{2} q^{*}}{E}, \\
\dot{q}^{*}=-\left(\partial_{\theta} h^{2}\right) h^{-2} E .
\end{gathered}
$$

Multiplying (10.39) by $q^{*}$ given by (10.38) we obtain

$$
\begin{aligned}
\frac{1}{2} \frac{\mathrm{d}}{\mathrm{d} t}\left(q^{*}\right)^{2} & =q^{*} \dot{q}^{*}=-\left(\partial_{\theta} h^{2}\right) h^{-6} a_{0}^{-2} E^{2} \dot{\theta}=-\frac{1}{2}\left(\partial_{\theta} h^{4}\right) h^{-8} a_{0}^{-2} E^{2} \dot{\theta} \\
& =\frac{1}{2} \frac{\left(r^{2}+a^{2}\right)^{2}}{\sigma^{4}}\left(\partial_{\theta} a^{2} \Delta \cos ^{2} \theta\right) \frac{\left(r^{2}+a^{2}\right)^{2}}{\Delta} \frac{\sigma^{4}}{\left(r^{2}+a^{2}\right)^{4}} E^{2} \dot{\theta} \\
& =\frac{1}{2} \frac{\mathrm{d}}{\mathrm{d} t} a^{2} E^{2} \cos ^{2} \theta \\
& \Longrightarrow\left|q^{*}\right|^{2}=\left|q_{0}^{*}\right|^{2}+a^{2} E^{2}\left(\cos ^{2} \theta-\cos ^{2} \theta_{0}\right),
\end{aligned}
$$

in particular $\mathcal{K}=\left|q^{*}\right|^{2}+a^{2} E^{2} \sin ^{2} \theta=$ Const. We have

$$
E^{2}=h^{4}\left(\xi^{2} h^{-4}+2 a_{0}^{2} \ell^{\prime} \xi q+a_{0}^{2} q^{2}\right) \Longrightarrow \frac{\xi^{2}}{E^{2}}=1-\frac{2 h^{4} a_{0}^{2} \ell^{\prime} \xi q}{E^{2}}-\frac{a_{0}^{2} h^{4} q^{2}}{E^{2}},
$$

in particular

$$
\frac{\xi_{0}^{2}}{E^{2}}=1+\Theta\left(L^{-1}\right)
$$

Using (10.41) we see that

$$
\frac{q_{0}^{*}}{E}=\frac{q_{0}}{E}+a \cos \theta_{0} \frac{\xi_{0}}{E}=a \cos \theta_{0}+\vartheta\left(L^{-1}\right) .
$$

Therefore

$$
\frac{\mathcal{K}}{E^{2}}=a^{2}+\vartheta\left(L^{-1}\right)
$$

These estimates are uniform for $\left(\widehat{r}_{0}, \theta_{0}, \xi_{0}, q_{\theta 0}\right) \in \mathcal{E}_{L} \cap \mathcal{J}_{L}^{t_{0}}$. We note that

$$
\left|\xi^{*}\right|^{2}=E^{2}\left(1-\frac{\Delta \mathcal{K}}{\left(r^{2}+a^{2}\right)^{2} E^{2}}\right), \quad\left|q^{*}\right|^{2}=\mathcal{K}-a^{2} E^{2} \sin ^{2} \theta .
$$

Using (10.43) and (10.44) we see that for $L$ sufficiently large $\xi^{*}$ does not change its sign. We now claim that there exists a constant $C\left(t_{0}\right)$ such that

$$
\dot{\hat{r}} \geq 1-C\left(t_{0}\right) L^{-\frac{1}{2}}
$$

uniformly in $\left(\widehat{r}_{0}, \theta_{0}, \xi_{0}, q_{\theta 0}\right) \in \mathcal{J}_{L}^{t_{0}} \cap \mathcal{E}_{L}$. We first argue that (10.45) proves the lemma. By (3.54) we see that for $t_{0}$ sufficiently large we have

$$
\widehat{z}\left(t_{0}, \theta\right)<-t_{0}, \quad \forall \theta \in[0, \pi] .
$$


If $t_{0}$ is fixed in this way, then there exists $\delta>0$ such that

$$
\dot{\widehat{z}}(\tau, \theta)>-1+\frac{2 \delta}{t_{0}}, \quad \forall 0 \leq \tau \leq t_{0} .
$$

We have

$$
\widehat{r}(0)-\widehat{z}\left(t_{0}, \theta\right) \geq-L^{-1}-t_{0}-\widehat{z}\left(t_{0}, \theta\right)>0, \quad \forall \theta \in[0, \pi]
$$

for $L$ sufficiently large and

$$
\frac{\mathrm{d}}{\mathrm{d} s}\left(\widehat{r}(s)-\widehat{z}\left(t_{0}-s, \theta\right)\right) \geq \frac{2 \delta}{t_{0}}-C\left(t_{0}\right) L^{-\frac{1}{2}}>\frac{\delta}{t_{0}}
$$

for $L$ sufficiently large. It follows

$$
\widehat{r}(s) \geq \widehat{z}\left(t_{0}-s, \theta\right)+\delta, \quad \forall 0 \leq s \leq t_{0} .
$$

It remains to show $(10.45)$. We have

$$
\begin{aligned}
\dot{\hat{r}} & =h^{4} \sqrt{1-\frac{a^{2} \Delta}{\left(r^{2}+a^{2}\right)^{2}}} \sqrt{1-\frac{\mathcal{K} \Delta}{\left(r^{2}+a^{2}\right)^{2} E^{2}}}+a \cos \theta \frac{h^{4} a_{0}^{2}}{E} q^{*} \\
& =1+\sqrt{1-\frac{a^{2} \Delta}{\left(r^{2}+a^{2}\right)^{2}}}\left(\sqrt{1-\frac{\mathcal{K} \Delta}{\left(r^{2}+a^{2}\right)^{2} E^{2}}}-\sqrt{1-\frac{a^{2} \Delta}{\left(r^{2}+a^{2}\right)^{2}}}\right) h^{4} \\
& +a \cos \theta h^{4} a_{0}^{2}\left(\frac{q^{*}}{E}-a \cos \theta\right) \\
& \geq 1-\theta\left(L^{-1}\right)+a \cos \theta h^{4} a_{0}^{2}\left(\frac{q^{*}}{E}-a \cos \theta\right),
\end{aligned}
$$

where we have used (10.43). It is therefore sufficient to show

$$
\left|a \cos \theta\left(\frac{q^{*}}{E}-a \cos \theta\right)\right|=\theta\left(L^{-\frac{1}{2}}\right) .
$$

We distinguish two cases:

$$
\begin{aligned}
& \text { 1) } \forall 0 \leq s \leq t_{0}, \quad \cos ^{2} \theta(s)>L^{-\frac{1}{2}} ; \\
& \text { 2) } \exists 0 \leq s_{0} \leq t_{0}, \quad \cos ^{2} \theta\left(s_{0}\right) \leq L^{-\frac{1}{2}}, \\
& s_{0} \neq 0 \Longrightarrow \cos ^{2} \theta(s)>L^{-\frac{1}{2}}, \quad \forall 0 \leq s<s_{0} .
\end{aligned}
$$

We will treat only the second case. The first case can be considered in some sense as a special case of the second one with $s_{0}=t_{0}$. We first suppose $s_{0}>0$. From (10.42), (10.49) we infer

$$
\operatorname{sign} q_{0}^{*}=\operatorname{sign}\left(\cos \theta_{0}\right)
$$

for $L$ sufficiently large and from (10.49) we infer, using also (10.43) and (10.44), that

$$
\left|q^{*}\right|^{2} \geq a^{2} E^{2} L^{-\frac{1}{2}}\left(1-\vartheta\left(L^{-\frac{1}{2}}\right)\right)>0
$$

if $L$ sufficiently large. Therefore

$$
\operatorname{sign}\left(q^{*}(s)\right)=\operatorname{sign} \cos \theta(s), \quad \forall 0 \leq s \leq s_{0}
$$


because neither $q^{*}(s)$ nor $\cos \theta(s)$ can change its sign on $\left[0, s_{0}\right]$. We find with $(10.43)$ and (10.44):

$$
\begin{aligned}
& \forall 0 \leq s \leq s_{0}, \quad \frac{q^{*}}{E}= \operatorname{sign}(\cos \theta) \sqrt{a^{2} \cos ^{2} \theta+\Theta\left(L^{-1}\right)} \\
& \Longrightarrow\left|\frac{q^{*}}{E}-a \cos \theta\right|=\vartheta\left(L^{-\frac{1}{2}}\right), \quad \forall 0 \leq s \leq s_{0} .
\end{aligned}
$$

We now claim that

$$
\forall s_{0} \leq s \leq t_{0}, \quad \cos ^{2} \theta(s) \leq C\left(t_{0}\right) L^{-\frac{1}{2}} .
$$

Indeed using (10.38), (10.43) and (10.44) we can estimate

$$
\begin{aligned}
\frac{\mathrm{d}}{\mathrm{d} t} a^{2} \cos ^{2} \theta & =-2 a^{2} \cos \theta \sin \theta \dot{\theta} \leq 2 a A_{1}|\cos \theta| \sqrt{\frac{\mathcal{K}}{E^{2}}-a^{2} \sin ^{2} \theta} \\
& \leq 2 a^{2} A_{1} \cos ^{2} \theta+\theta\left(L^{-\frac{1}{2}}\right),
\end{aligned}
$$

where $A_{1}:=\max _{r \geq r_{+}} h^{4} a_{0}^{2} a$. By the Gronwall lemma we obtain

$$
\cos ^{2} \theta \leq \int_{s_{0}}^{s} \mathrm{e}^{2 A_{1}(s-\tau)} \theta\left(L^{-\frac{1}{2}}\right) \mathrm{d} \tau+\mathrm{e}^{2 A_{1}\left(s-s_{0}\right)} \cos ^{2} \theta\left(s_{0}\right) \leq \widetilde{C}\left(t_{0}\right) L^{-\frac{1}{2}} .
$$

Inequality (10.50) follows and therefore

$$
|a \cos \theta|=\Theta\left(L^{-\frac{1}{4}}\right), \quad\left|\frac{q^{*}}{E}\right|=\Theta\left(L^{-\frac{1}{4}}\right),
$$

i.e. (10.46). If $s_{0}=0$ we can start with (10.50).

\subsection{Proof of Proposition 10.1}

Let us first show the following lemma:

Lemma 10.7. - We have

$$
\begin{aligned}
& \forall N, R>0, \exists C_{N, R}>0, t_{0}>0 \\
& \forall t_{\epsilon} \geq t_{0}, \exists \delta=\delta\left(t_{\epsilon}, N, R\right)>0, T_{0}=T_{0}\left(N, R, t_{\epsilon}\right)>0, \forall 0 \leq s \leq t_{\epsilon}, T \geq T_{0} \\
& \left\|\left(1-\phi_{\delta}\right)\left(.-\widehat{z}\left(t_{\epsilon}-s, \theta\right)\right) \mathrm{e}^{-i s H} F_{t_{\epsilon}}^{T}\right\| \leq C_{N, R} \mathrm{e}^{-\kappa_{+} t_{\epsilon}} .
\end{aligned}
$$

Proof. - Because of the finite propagation speed we can replace $1-\phi_{\delta}\left(.-\widehat{z}\left(t_{\epsilon}-s, \theta\right)\right)$ by $\chi_{\epsilon}\left(1-\phi_{\delta}\left(.-\widehat{z}\left(t_{\epsilon}-s, \theta\right)\right)\right)$ with $\chi_{\epsilon} \in C^{\infty}(\mathbb{R})$ such that

$$
\chi_{\epsilon}(\widehat{r})= \begin{cases}0 & \widehat{r} \leq-4 t_{\epsilon} \\ 1 & \widehat{r} \geq-3 t_{\epsilon} .\end{cases}
$$

By the results of the preceding sections it is sufficient to show

$$
\chi_{\epsilon}\left(1-\phi_{\delta}\right)\left(.-\widehat{z}\left(t_{\epsilon}-s, \theta\right)\right) \mathrm{e}^{-i s H_{d}} \mathrm{Op}\left(N_{\leftarrow}^{*}\right) F_{t_{\epsilon}}^{T} \longrightarrow \infty, \quad T \rightarrow \infty .
$$

Here we have used (10.34) and that

$$
\left[\chi_{\epsilon}\left(1-\phi_{\delta}\right)\left(.-\widehat{z}\left(t_{\epsilon}-s, \theta\right)\right), \mathrm{Op}(W) M\right] \in \Psi^{-1}\left(\Sigma_{1}\right) .
$$


Using Lemma 10.3 we can replace $\mathrm{Op}\left(N_{\leftarrow}^{*}\right) F_{t_{\epsilon}}^{T}$ by $\mathrm{Op}\left(N_{\leftarrow}^{*} \chi_{L}(|\xi / q|)\right) F_{t_{\epsilon}}^{T(1)}$ with $\chi_{L} \in$ $C^{\infty}(\mathbb{R})$,

$$
\chi_{L}(x)= \begin{cases}1 & |x| \geq 2 L \\ 0 & |x| \leq L\end{cases}
$$

Let $\tilde{\chi}_{\epsilon} \in C_{0}^{\infty}(\mathbb{R})$ and

$$
\begin{array}{lll}
\tilde{\chi}_{\epsilon}=1 & \text { on } & \operatorname{supp} F_{t_{\epsilon}}^{T}, \\
\tilde{\chi}_{\epsilon}=0 & \text { on } & \left(-\infty,-t_{\epsilon}-L^{-1}\right)
\end{array}
$$

for all $T \geq T_{0}$. By [31, Proposition 18.1.26] we have for all $f \in L^{2}(\mathbb{R} \times[0, \pi])$ :

$$
\begin{aligned}
\mathrm{WF}\left(\operatorname{Op}\left(j_{+}(\xi /|q|) \chi\left(h^{2} \sqrt{k^{\prime 2} \xi^{2}+a_{0}^{2}\left(\ell^{\prime} \xi+q\right)^{2}}\right) \chi_{L}(|\xi / q|)\right) \tilde{\chi}_{\epsilon} f\right) \\
\subset\left\{(\widehat{r}, \omega ; \xi, q) ; \widehat{r} \geq-t_{\epsilon}-L^{-1},|\xi| \geq L|q|, \xi>0\right\}, \\
\mathrm{WF}\left(\operatorname{Op}\left(j_{-}(\xi /|q|) \chi\left(h^{2} \sqrt{k^{\prime 2} \xi^{2}+a_{0}^{2}\left(\ell^{\prime} \xi+q\right)^{2}}\right) \chi_{L}(|\xi / q|)\right) \tilde{\chi}_{\epsilon} f\right) \\
\subset\left\{(\widehat{r}, \omega ; \xi, q) ; \widehat{r} \geq-t_{\epsilon}-L^{-1},|\xi| \geq L|q|, \xi<0\right\},
\end{aligned}
$$

where WF denotes the wave front set (see [31, Chapter VIII]). Then by the classical results of propagation of singularities (see e.g. [31, Theorem 26.1.1]), Sobolev embeddings and Lemma 10.6, we find that the operators

$$
\begin{aligned}
\chi_{\epsilon}(1- & \left.\phi_{\delta}\right)(.-\widehat{z}(s, \theta)) \mathrm{e}^{-i s H_{d}} \\
& \times \mathrm{Op}\left(j_{+}(\xi /|q|) \chi\left(h^{2} \sqrt{k^{\prime 2} \xi^{2}+a_{0}^{2}\left(\ell^{\prime} \xi+q\right)^{2}}\right) \chi_{L}(|\xi / q|)\right) \tilde{\chi}_{\epsilon} P_{14}, \\
\chi_{\epsilon}(1- & \left.\phi_{\delta}\right)(.-\widehat{z}(s, \theta)) \mathrm{e}^{-i s H_{d}} \\
& \times \mathrm{Op}\left(j_{-}(\xi /|q|) \chi\left(h^{2} \sqrt{k^{\prime 2} \xi^{2}+a_{0}^{2}\left(\ell^{\prime} \xi+q\right)^{2}}\right) \chi_{L}(|\xi / q|)\right) \tilde{\chi}_{\epsilon} P_{23}
\end{aligned}
$$

are compact for $L, t_{\epsilon}$ sufficiently large. The lemma now follows from Corollary 10.1 and the observation that

$$
\left(F_{t_{\epsilon}}^{T}\right)_{2,3}=0, \quad\left(\operatorname{Op}\left(\left(N_{\leftarrow}^{-}\right)^{*}\right) F_{t_{\epsilon}}^{T}\right)_{1,4}=0, \quad \tilde{\chi}_{\epsilon} F_{t_{\epsilon}}^{T}=F_{t_{\epsilon}}^{T},
$$

for $T$ sufficiently large.

Proof of Proposition 10.1. - We first show that

$$
\left.\|\left(U\left(0, t_{\epsilon}\right)-\phi_{\delta}(.-\widehat{z}(0, \theta))\right) \mathrm{e}^{-i t_{\epsilon} H}\right) F_{t_{\epsilon}}^{T} \| \leq C_{N, R} t_{\epsilon} \mathrm{e}^{-\kappa_{+} t_{\epsilon}}
$$

uniformly in $T$ large for $\delta$ sufficiently small. Let $\phi_{\delta}(., t)=\phi_{\delta}(.-\widehat{z}(t, \theta))$. We define for $g=P_{1,4} g \in \mathscr{H}$ :

$$
v(t, \widehat{r}, \omega)=\left(U\left(t, t_{\epsilon}\right)-\phi_{\delta}(t) \mathrm{e}^{i\left(t-t_{\epsilon}\right) H}\right) g .
$$

(1) As in the definition of $W(\widehat{r}, \theta, \xi, q), \chi\left(h^{2} \sqrt{k^{\prime 2} \xi^{2}+a_{0}^{2}\left(\ell^{\prime} \xi+q\right)^{2}}\right) \chi_{L}(|\xi / q|)$ can be extended to a smooth function. 
If supp $g \subset\left(\widehat{z}\left(t_{\epsilon}, \theta\right)+\delta, \infty\right) \times S^{2}$, then $v$ is a solution of

$$
\left\{\begin{array}{l}
v\left(t_{\epsilon}, \widehat{r}, \omega\right)=0, \\
\partial_{t} v=i H_{t} v+h(t), \quad \widehat{r}>\widehat{z}(t, \theta), \\
\sum_{\mu \in\{t, \widehat{r}, \omega\}} \mathcal{N}_{\mu} \widehat{\gamma}^{\mu} v(t, \widehat{z}(t, \theta), \omega)=-i v(t, \widehat{z}(t, \theta), \omega),
\end{array}\right.
$$

where

Thus

$$
h(t)=-\left(\left(\frac{\mathrm{d}}{\mathrm{d} t} \phi_{\delta}(t)\right)+\left[\phi_{\delta}, H\right]\right) \mathrm{e}^{i\left(t-t_{\epsilon}\right) H} g=B(t) g .
$$

$$
v(0)=\int_{t_{\epsilon}}^{0} U(0, s) B(s) g \mathrm{~d} s .
$$

We obtain by Lemma 10.7 for $\delta$ small enough

$$
\left\|\left(U\left(0, t_{\epsilon}\right)-\phi_{\delta}(0) \mathrm{e}^{-i t_{\epsilon} H}\right) F_{\epsilon}^{T}\right\| \leq \int_{0}^{t_{\epsilon}}\left\|B(s) F_{t_{\epsilon}}^{T}\right\| \mathrm{d} s \leq C_{N, R} t_{\epsilon} \mathrm{e}^{-\kappa_{+} t_{\epsilon}}
$$

uniformly in $T$ large. We now estimate for $\epsilon>0$ given

$$
\begin{aligned}
& \left\|\mathcal{J}(\widehat{r}) U(0, T) f-\phi_{\delta}(.-\widehat{z}(0, \theta)) \mathrm{e}^{-i t_{\epsilon} H} F_{t_{\epsilon}}^{T}\right\| \\
& \leq\left\|\mathcal{J}(\widehat{r}) U\left(0, t_{\epsilon}\right)\left(U\left(t_{\epsilon}, T\right) f-U_{\leftarrow}\left(t_{\epsilon}, T\right) \Omega_{\leftarrow}^{-} f\right)\right\| \\
& \quad+\left\|\mathcal{J}(\widehat{r}) U\left(0, t_{\epsilon}\right)\left(U_{\leftarrow}\left(t_{\epsilon}, T\right)\left(\Omega_{\leftarrow}^{-} f\right)_{R}^{N}-F_{t_{\epsilon}}^{T}\right)\right\|+\left\|\left(\Omega_{\leftarrow}^{-} f\right)_{R}^{N}-\Omega_{\leftarrow}^{-} f\right\| \\
& \quad+\left\|\mathcal{J}(\widehat{r})\left(U\left(0, t_{\epsilon}\right)-\phi_{\delta}(.-\widehat{z}(0, \theta)) \mathrm{e}^{-i t_{\epsilon} H}\right) F_{t_{\epsilon}}^{T}\right\| \\
& <\left\|\mathcal{J}(\widehat{r}) U\left(0, t_{\epsilon}\right)\left(1-\mathcal{J}\left(\widehat{r}+t_{\epsilon}\right)\right) U\left(t_{\epsilon}, T\right) f\right\| \\
& \quad+\left\|\mathcal{J}(\widehat{r}) U\left(0, t_{\epsilon}\right)\left(1-\mathcal{J}\left(\widehat{r}+t_{\epsilon}\right)\right) U_{\leftarrow}\left(t_{\epsilon}, T\right) \Omega_{\leftarrow}^{-} f\right\|+\frac{1}{3} \epsilon
\end{aligned}
$$

fixing first $N, R$ and choosing then $t_{\epsilon}, T$ sufficiently large and $\delta=\delta\left(t_{\epsilon}, N, R\right)$ sufficiently small. Here we have used Proposition 9.1, Lemma 10.2 and (10.51). We now claim that for $t_{\epsilon}$ fixed the first two terms in (10.52) go to zero when $T$ goes to infinity. Indeed

$$
\begin{aligned}
\mathscr{J}(\widehat{r}) U( & \left.0, t_{\epsilon}\right)\left(1-\mathcal{J}\left(\widehat{r}+t_{\epsilon}\right)\right) U\left(t_{\epsilon}, T\right) f \\
= & \mathcal{J}(\widehat{r}) U\left(0, t_{\epsilon}\right)\left(1-\mathcal{J}\left(\widehat{r}+t_{\epsilon}\right)\right) \mathrm{e}^{i\left(t_{\epsilon}-T\right) H} f \\
= & \mathcal{J}(\widehat{r}) U\left(0, t_{\epsilon}\right)\left(1-\mathcal{J}\left(\widehat{r}+t_{\epsilon}\right)\right) \mathrm{e}^{i\left(t_{\epsilon}-T\right) H} \mathbf{1}_{(-\infty, 0]}\left(P^{-}\right) f \\
& \quad+\mathcal{J}(\widehat{r}) U\left(0, t_{\epsilon}\right)\left(1-\mathcal{J}\left(\widehat{r}+t_{\epsilon}\right)\right) \mathrm{e}^{i\left(t_{\epsilon}-T\right) H} \mathbf{1}_{[0, \infty)}\left(P^{-}\right) f .
\end{aligned}
$$

We can suppose $f=\chi(H) f, \chi \in C_{0}^{\infty}(\mathbb{R})$, supp $\chi \subset\left\{-m+\eta^{n}, m+\eta^{n}\right\}$. By the minimal velocity estimate we can replace $\mathbf{1}_{(-\infty, 0)}\left(P^{-}\right)$by $\mathbf{1}_{\left(-\infty,-\epsilon_{\chi}\right)}\left(P^{-}\right)$and $\mathbf{1}_{[0, \infty)}\left(P^{-}\right)$by $\mathbf{1}_{[1-\tilde{\epsilon}, \infty)}\left(P^{-}\right)$, where $\tilde{\epsilon}>0$ and $\epsilon_{\chi}$ is given by Lemma 6.2. But,

$$
\begin{aligned}
\lim _{T \rightarrow \infty} \mathcal{J}(\widehat{r}) U\left(0, t_{\epsilon}\right)\left(1-\mathcal{J}\left(\widehat{r}+t_{\epsilon}\right)\right) \mathrm{e}^{i\left(t_{\epsilon}-T\right) H} \mathbf{1}_{\left(-\infty,-\epsilon_{\chi}\right]}\left(P^{-}\right) f \\
\quad=\lim _{T \rightarrow \infty} \mathcal{J}(\widehat{r}) U\left(0, t_{\epsilon}\right)\left(1-\mathcal{J}\left(\widehat{r}+t_{\epsilon}\right)\right) \mathbf{1}_{\left(-\infty,-\epsilon_{\chi}\right]}\left(\frac{\widehat{r}}{t_{\epsilon}-T}\right) \mathrm{e}^{i\left(t_{\epsilon}-T\right) H} f=0
\end{aligned}
$$


by the finite propagation speed for $U\left(0, t_{\epsilon}\right)$. In a similar way we find

$$
\begin{aligned}
\lim _{T \rightarrow \infty} \mathcal{J}(\widehat{r}) U\left(0, t_{\epsilon}\right)\left(1-\mathcal{J}\left(\widehat{r}+t_{\epsilon}\right)\right) \mathrm{e}^{i\left(t_{\epsilon}-T\right) H} \mathbf{1}_{[1-\tilde{\epsilon}, \infty)}\left(P^{-}\right) f \\
\quad=\lim _{T \rightarrow \infty} \mathcal{J}(\widehat{r}) U\left(0, t_{\epsilon}\right)\left(1-\mathcal{J}\left(\widehat{r}+t_{\epsilon}\right)\right) \mathbf{1}_{[1-\tilde{\epsilon}, \infty)}\left(\frac{\widehat{r}}{t_{\epsilon}-T}\right) \mathrm{e}^{i\left(t_{\epsilon}-T\right) H}=0
\end{aligned}
$$

because $\left(1-\mathcal{J}\left(\widehat{r}+t_{\epsilon}\right)\right) \mathbf{1}_{[1-\tilde{\epsilon}, \infty)}\left(\widehat{r} /\left(t_{\epsilon}-T\right)\right)=0$ for $T$ sufficiently large. The second term can be treated in a similar way. This concludes the proof of the proposition. 



\section{CHAPTER 11}

\section{PROOF OF THE MAIN THEOREM}

In this chapter we put the results of the previous chapters together to prove the main theorem. Recall that we are working with the operators $H_{\eta^{n}}^{\nu, n}$, etc. and that the indices are suppressed. All operators are considered as acting on $\mathcal{H}^{n}$.

\subsection{The energy cut-off}

In this section $N, R>0$ will be fixed. Let

$$
\Sigma_{0}^{-}=\{(\widehat{r}, \omega) ; \widehat{r} \leq \widehat{z}(0, \theta)\}, \quad \mathcal{H}_{0}^{-}=\left(L^{2}\left(\Sigma_{0}^{-}, \mathrm{d} \widehat{r} \mathrm{~d} \omega\right)\right)^{4} .
$$

On $\mathcal{H}_{0}^{-}$we define

$$
\begin{aligned}
H_{0}^{-} & =H, \\
D\left(H_{0}^{-}\right) & =\left\{u \in \mathcal{H}_{0}^{-} ; H u \in \mathcal{H}_{0}^{-},\right. \\
& \left.\sum_{\hat{\mu} \in\{(t, \widehat{r}, \theta, \varphi)\}} \mathcal{N}_{\hat{\mu}} \widehat{\gamma}^{\hat{\mu}} \Psi(\widehat{z}(0, \theta), \omega)=-i \Psi(0, \widehat{z}(0, \theta), \omega)\right\} .
\end{aligned}
$$

We need the following:

Lemma 11.1. - (i) Let $\phi \in C^{\infty}\left(\mathbb{R} \times S^{2}\right), \partial_{\widehat{r}} \phi \in C_{0}^{\infty}\left(\mathbb{R} \times S^{2}\right), \phi \equiv 0$ on $\Sigma_{0}^{-}$and $\chi \in \phi^{0}(\mathbb{R})$. Then $\left(\chi\left(H_{0}^{-} \oplus H_{0}\right)-\chi(H)\right) \phi$ is compact.

(ii) Let $\phi \in C_{0}^{\infty}\left(\Sigma_{0}\right), \chi \in \phi^{-1}(\mathbb{R})$. Then $\chi\left(H_{0}^{-} \oplus H_{0}\right) \phi, \chi(H) \phi$ are compact.

Proof. - (i) We write $\chi(x)=\widehat{\chi}(x)(x+i), \widehat{\chi} \in \phi^{-1}$. We have as an identity between bounded operators

$$
\chi\left(H_{0}^{-} \oplus H_{0}\right) \phi=\widehat{\chi}\left(H_{0}^{-} \oplus H_{0}\right)(H+i) \phi .
$$

Therefore

$$
\begin{aligned}
&\left(\chi\left(H_{0}^{-} \oplus H_{0}\right)-\chi(H)\right) \phi=\left(\widehat{\chi}\left(H_{0}^{-} \oplus H_{0}\right)-\widehat{\chi}(H)\right)(H+i) \phi \\
&=\left(\widehat{\chi}\left(H_{0}^{-} \oplus H_{0}\right)-\widehat{\chi}(H)\right) \widetilde{\phi}(H+i) \phi \\
&+\left(\widehat{\chi}\left(H_{0}^{-} \oplus H_{0}\right)-\widehat{\chi}(H)\right)[H, \widetilde{\phi}] \phi,
\end{aligned}
$$


where $\widetilde{\phi} \in C^{\infty}\left(\mathbb{R} \times S^{2}\right), \widetilde{\phi} \phi=\phi, \widetilde{\phi} \equiv 0$ on $\Sigma_{0}^{-}$. We only show that the first term is compact, the second term can be treated in a similar manner. Let $\tilde{\chi}$ be an almost analytic extension of $\widehat{\chi}$ with

$$
\begin{aligned}
& \left.\tilde{\chi}\right|_{\mathbb{R}}=\widehat{\chi}, \\
& \forall N,|\bar{\partial} \widetilde{\chi}(z)| \leq C_{N}|\Im z|^{N}\langle\Re z\rangle^{-2-N}, \quad \operatorname{supp} \tilde{\chi} \subset\{x+i y ;|y| \leq C\langle x\rangle\} .
\end{aligned}
$$

By the Helffer-Sjöstrand formula we can write

$$
\begin{aligned}
\left(\widehat{\chi}\left(H_{0}^{-} \oplus H_{0}\right)\right. & -\widehat{\chi}(H)) \widetilde{\phi}(H+i) \\
& =\int \bar{\partial} \widetilde{\chi}(z)\left(\left(z-H_{0}^{-} \oplus H_{0}\right)^{-1}-(z-H)^{-1}\right) \widetilde{\phi}(H+i) \mathrm{d} z \wedge \mathrm{d} \bar{z} .
\end{aligned}
$$

This identity is first understood as an identity between operators $D(H) \rightarrow \mathcal{H}$. Now,

$$
\begin{gathered}
\bar{\partial} \widetilde{\chi}(z)\left(\left(z-H_{0}^{-} \oplus H_{0}\right)^{-1}-(z-H)^{-1}\right) \widetilde{\phi}(H+i) \\
=\bar{\partial} \widetilde{\chi}(z)\left(\left(z-H_{0}^{-} \oplus H_{0}\right)^{-1}(z-H) \widetilde{\phi}(z-H)^{-1}\right. \\
+\left(z-H_{0}^{-} \oplus H_{0}\right)^{-1}[H, \widetilde{\phi}](z-H)^{-1} \\
\left.\quad-\widetilde{\phi}(z-H)^{-1}-(z-H)^{-1}[H, \widetilde{\phi}](z-H)^{-1}\right)(H+i) \\
=\bar{\partial} \widetilde{\chi}(z)\left(\left(z-H_{0}^{-} \oplus H_{0}\right)^{-1}[H, \widetilde{\phi}](z-H)^{-1}\right. \\
\left.-(z-H)^{-1}[H, \widetilde{\phi}](z-H)^{-1}\right)(H+i) .
\end{gathered}
$$

Both terms are compact and can be estimated by $C\langle x\rangle^{-3}$ according to (11.2). This shows first that the identity (11.3) can be extended to an identity between bounded operators and then that the operator is compact.

(ii) The fact that $\chi(H) \phi$ is compact follows from the estimates $(4.43),(4.44)$ and the same arguments as in [29, Corollary 4.2]. This entails that $\chi\left(H_{0}^{-} \oplus H_{0}\right) \phi$ is compact by part (i) of the lemma.

Let now $\delta=\delta\left(t_{\epsilon}\right)$ and $\phi_{\delta}$ be as in Proposition 10.1, $\widetilde{\phi}_{\delta}(\widehat{r}, \theta)=\phi_{\delta}(\widehat{r}-\widehat{z}(0, \theta))$ and $\chi_{1}, \chi_{2} \in C^{\infty}(\mathbb{R})$ such that

$$
\chi_{1}(x)=\left\{\begin{array}{ll}
1 & |x| \leq 1+\eta, \\
0 & |x| \geq 2+\eta,
\end{array} \quad \chi_{2}(x)= \begin{cases}0 & x \leq 1+\eta, \\
1 & x \geq 2+\eta,\end{cases}\right.
$$

and such that $\chi_{1}(x)+\chi_{2}(x)=1$ for all $x \geq 0$. Here and in the following $\eta=\eta^{n}$. Then we have by Lemma 11.1 and Corollary 10.1:

$$
\lim _{T \rightarrow \infty}\left\|\chi_{1}\left(H_{0}\right) \widetilde{\phi}_{\delta} \mathrm{e}^{-i t_{\epsilon} H} F_{t_{\epsilon}}^{T}\right\|_{0}^{2}=0
$$


Indeed we can replace $\widetilde{\phi}_{\delta}$ by a compactly supported function using the support property (10.2) of $F_{t_{\epsilon}}^{T}$. Furthermore we have

$$
\begin{aligned}
& \left\|\chi_{2}\left(H_{0}\right) \widetilde{\phi}_{\delta} \mathrm{e}^{-i t_{\epsilon} H} F_{t_{\epsilon}}^{T}\right\|_{0}-\left\|\chi_{1}\left(H_{0}\right) \widetilde{\phi}_{\delta} \mathrm{e}^{-i t_{\epsilon} H} F_{t_{\epsilon}}^{T}\right\|_{0} \\
& \leq\left\|\mathbf{1}_{[\eta, \infty)}\left(H_{0}\right) \widetilde{\phi}_{\delta} \mathrm{e}^{-i t_{\epsilon} H} F_{t_{\epsilon}}^{T}\right\|_{0} \\
& \quad \leq\left\|\chi_{1}\left(H_{0}\right) \widetilde{\phi}_{\delta} \mathrm{e}^{-i t_{\epsilon} H} F_{t_{\epsilon}}^{T}\right\|_{0}+\left\|\chi_{2}\left(H_{0}\right) \widetilde{\phi}_{\delta} \mathrm{e}^{-i t_{\epsilon} H} F_{t_{\epsilon}}^{T}\right\|_{0} .
\end{aligned}
$$

Using (11.4), (11.5) and Lemma 11.1 we obtain

$$
\begin{aligned}
\lim _{T \rightarrow \infty}\left\|\mathbf{1}_{[\eta, \infty)}\left(H_{0}\right) \widetilde{\phi}_{\delta} \mathrm{e}^{-i t_{\epsilon} H} F_{t_{\epsilon}}^{T}\right\|_{0} & =\lim _{T \rightarrow \infty}\left\|\chi_{2}\left(H_{0}\right) \widetilde{\phi}_{\delta} \mathrm{e}^{-i t_{\epsilon} H} F_{t_{\epsilon}}^{T}\right\|_{0} \\
& =\lim _{T \rightarrow \infty}\left\|\chi_{2}\left(H_{0}^{-} \oplus H_{0}\right) \widetilde{\phi}_{\delta} \mathrm{e}^{-i t_{\epsilon} H}\left(0 \oplus F_{t_{\epsilon}}^{T}\right)\right\| \\
& =\lim _{T \rightarrow \infty}\left\|\chi_{2}(H) \widetilde{\phi}_{\delta} \mathrm{e}^{-i t_{\epsilon} H} F_{t_{\epsilon}}^{T}\right\| .
\end{aligned}
$$

LEMMA 11.2. - Let $\chi \in \phi^{0}(\mathbb{R}), f \in D\left(\left\langle\not D_{S^{2}}\right\rangle\right)$. Let $\tilde{\chi}$ be an almost analytic extension of $\chi$ with

$$
\left.\widetilde{\chi}\right|_{\mathbb{R}}=\chi, \quad \forall N, \quad|\bar{\partial} \widetilde{\chi}(z)| \leq C_{N}|\Im z|^{N}\langle\Re z\rangle^{-1-N}, \quad \operatorname{supp} \tilde{\chi} \subset\{x+i y ;|y| \leq C\langle\Re z\rangle\} .
$$

Then we have

$$
\left(\chi(H)-\chi\left(H_{\leftarrow}\right)\right) f=\int \bar{\partial} \widetilde{\chi}(z)(z-H)^{-1}\left(H-H_{\leftarrow}\right)\left(z-H_{\leftarrow}\right)^{-1} f \mathrm{~d} z \wedge \mathrm{d} \bar{z} .
$$

REMARK 11.1. - Note that neither

$$
\int \bar{\partial} \widetilde{\chi}(z)(z-H)^{-1} f \mathrm{~d} z \wedge \mathrm{d} \bar{z} \quad \text { nor } \quad \int \bar{\partial} \widetilde{\chi}(z)\left(z-H_{\leftarrow}\right)^{-1} f \mathrm{~d} z \wedge \mathrm{d} \bar{z}
$$

is convergent in $\mathscr{H}$, but the R.H.S. of (11.7) is.

Proof. - Let $\chi_{0} \in C_{0}^{\infty}(\mathbb{R})$ with $\chi_{0}=1$ in a neighborhood of 0 and $\chi_{0}^{m}(x)=\chi_{0}(x / m)$.

Let $\widetilde{\chi}_{0} \in C_{0}^{\infty}(\mathbb{C})$ be an almost analytic extension of $\chi_{0}$ with

$$
\left|\bar{\partial} \widetilde{\chi}_{0}\right| \leq C_{N}|\Im z|^{N}, \quad \forall N
$$

Then $\tilde{\chi}_{0}(z / m)$ is an almost analytic extension of $\chi_{0}^{m}$. Clearly

$$
\lim _{m \rightarrow \infty} \chi(H) \chi_{0}^{m}(H) f=\chi(H) f, \quad \lim _{m \rightarrow \infty} \chi\left(H_{\leftarrow}\right) \chi_{0}^{m}\left(H_{\leftarrow}\right) f=\chi\left(H_{\leftarrow}\right) f .
$$

We have

$$
\chi(H) \chi_{0}^{m}(H)=\int \bar{\partial}\left(\widetilde{\chi}(z) \widetilde{\chi}_{0}\left(\frac{z}{m}\right)\right)(z-H)^{-1} \mathrm{~d} z \wedge \mathrm{d} \bar{z}
$$


and the R.H.S. of (11.8) is convergent in norm for all $m$. Therefore

$$
\begin{aligned}
\left(\chi(H) \chi_{0}^{m}(H)-\chi\left(H_{\leftarrow}\right) \chi_{0}^{m}\left(H_{\leftarrow}\right)\right) f & \\
= & \int \bar{\partial}\left(\widetilde{\chi}(z) \widetilde{\chi}_{0}\left(\frac{z}{m}\right)\right)(z-H)^{-1}\left(H-H_{\leftarrow}\right)\left(z-H_{\leftarrow}\right)^{-1} f \mathrm{~d} z \wedge \mathrm{d} \bar{z} \\
= & \int(\bar{\partial} \tilde{\chi}(z)) \widetilde{\chi}_{0}\left(\frac{z}{m}\right)(z-H)^{-1}\left(H-H_{\leftarrow}\right)\left(z-H_{\leftarrow}\right)^{-1} f \mathrm{~d} z \wedge \mathrm{d} \bar{z} \\
& \quad+\int \tilde{\chi}(z)\left(\bar{\partial} \widetilde{\chi}_{0}\right)\left(\frac{z}{m}\right) \frac{1}{m}(z-H)^{-1}\left(H-H_{\leftarrow}\right)\left(z-H_{\leftarrow}\right)^{-1} f \mathrm{~d} z \wedge \mathrm{d} \bar{z} \\
= & : I_{1}+I_{2} .
\end{aligned}
$$

We have

$$
\left\|\bar{\partial} \widetilde{\chi}(z) \widetilde{\chi}_{0}\left(\frac{z}{m}\right)(z-H)^{-1}\left(H-H_{\leftarrow}\right)\left(z-H_{\leftarrow}\right)^{-1} f\right\| \lesssim\langle x\rangle^{-3}\left\|\left\langle D_{S^{2}}\right\rangle f\right\|
$$

uniformly in $m$ and for all $z \in \mathbb{C} \backslash\left(\sigma(H) \cup \sigma\left(H_{\leftarrow}\right)\right)$ :

$$
\begin{aligned}
(\bar{\partial} \tilde{\chi}(z)) \tilde{\chi}_{0}\left(\frac{z}{m}\right)(z-H)^{-1}\left(H-H_{\leftarrow}\right)\left(z-H_{\leftarrow}\right)^{-1} f & \\
& \longrightarrow(\bar{\partial} \widetilde{\chi}(z))(z-H)^{-1}\left(H-H_{\leftarrow}\right)\left(z-H_{\leftarrow}\right)^{-1} f .
\end{aligned}
$$

Then by the Lebesgue Theorem $I_{1}$ converges to the R.H.S. of (11.7). The change of coordinates $u=z / m$ gives

$$
I_{2}=\int m \tilde{\chi}(u m) \bar{\partial} \widetilde{\chi}_{0}(u)(u m-H)^{-1}\left(H-H_{\leftarrow}\right)\left(u m-H_{\leftarrow}\right)^{-1} f \mathrm{~d} u \wedge \mathrm{d} \bar{u} .
$$

As $\tilde{\chi}(z)$ is bounded we can estimate

$$
\begin{aligned}
\left\|m \tilde{\chi}(u m) \bar{\partial} \widetilde{\chi}_{0}(u)(u m-H)^{-1}\left(H-H_{\leftarrow}\right)\left(u m-H_{\leftarrow}\right)^{-1} f\right\| & \\
& \lesssim|\Im u|^{2} \frac{m}{m^{2}|\Im u|^{2}}\left\|\left\langle D_{S^{2}}\right\rangle f\right\|=\frac{\left\|\left\langle D_{S^{2}}\right\rangle f\right\|}{m} \longrightarrow 0, \quad m \rightarrow \infty .
\end{aligned}
$$

Thus $I_{2} \rightarrow 0$ as $m \rightarrow \infty$.

LEMMA 11.3. - We have

$$
\begin{aligned}
& \forall \epsilon>0, \exists t_{0}>0, \forall t_{\epsilon} \geq t_{0}, \exists T_{0}=T_{0}\left(t_{\epsilon}\right), \forall T \geq T_{0}, \\
& \quad\left|\left\|\chi_{2}(H) \widetilde{\phi}_{\delta\left(t_{\epsilon}\right)} \mathrm{e}^{-i t_{\epsilon} H} F_{t_{\epsilon}}^{T}\right\|-\left\|\mathbf{1}_{[0, \infty)}\left(H_{\leftarrow}\right) F_{t_{0}}^{T}\right\|\right|<\epsilon .
\end{aligned}
$$

Here $\delta\left(t_{\epsilon}\right)$ is chosen as in Proposition 10.1.

Proof. - Using Lemma 10.7 we see that

$$
\begin{aligned}
& \forall \epsilon>0, \exists t_{0}>0, \forall t_{\epsilon} \geq t_{0}, \exists T_{0}=T_{0}\left(t_{\epsilon}\right), \forall T \geq T_{0}, \\
& \left|\left\|\chi_{2}(H) \widetilde{\phi}_{\delta\left(t_{\epsilon}\right)} \mathrm{e}^{-i t_{\epsilon} H} F_{t_{\epsilon}}^{T}\right\|-\left\|\chi_{2}(H) \mathrm{e}^{-i t_{\epsilon} H} F_{t_{\epsilon}}^{T}\right\|\right|<\epsilon .
\end{aligned}
$$

We then have to show that

$$
\left(\chi_{2}(H)-\chi_{2}\left(H_{\leftarrow}\right)\right) F_{t_{\epsilon}}^{T} \longrightarrow 0, \quad T \rightarrow 0 .
$$


To this purpose let $\tilde{\chi}_{2}$ be an almost analytic extension of $\chi_{2}$ with

$$
\left.\tilde{\chi}_{2}\right|_{\mathbb{R}}=\chi, \quad \forall N,\left|\bar{\partial} \widetilde{\chi}_{2}(z)\right| \leq C_{N}|\Im z|^{N}\langle\Re z\rangle^{-1-N}, \operatorname{supp} \tilde{\chi}_{2} \subset\{x+i y ;|y| \leq C\langle x\rangle\} .
$$

Then we have by Lemma 11.2

$$
\begin{aligned}
\left(\chi_{2}(H)\right. & \left.-\chi_{2}\left(H_{\leftarrow}\right)\right) F_{t_{\epsilon}}^{T} \\
& =\int \bar{\partial} \widetilde{\chi}_{2}(z)(z-H)^{-1}\left(H-H_{\leftarrow}\right)\left(z-H_{\leftarrow}\right)^{-1} F_{t_{\epsilon}}^{T} \mathrm{~d} z \wedge \mathrm{d} \bar{z} \\
& =\int \bar{\partial} \widetilde{\chi}_{2}(z)(z-H)^{-1}\left(P_{\omega}+W\right)\left\langle\not_{S^{2}}\right\rangle^{-1}\left(z-H_{\leftarrow}\right)^{-1}\left\langle\not_{S^{2}}\right\rangle F_{t_{\epsilon}}^{T} \mathrm{~d} z \wedge \mathrm{d} \bar{z}
\end{aligned}
$$

By Lemma 10.3 we find

$$
\left(z-H_{\leftarrow}\right)^{-1}\left\langle\not D_{S^{2}}\right\rangle F_{t_{\epsilon}}^{T} \longrightarrow 0, \quad T \rightarrow \infty
$$

and we have

$$
\left\|\bar{\partial} \widetilde{\chi}_{2}(z)(z-H)^{-1}\left(P_{\omega}+W\right)\left(z-H_{\leftarrow}\right)^{-1} F_{t_{\epsilon}}^{T}\right\| \lesssim\langle x\rangle^{-3}\left\|\left\langle\not D_{S^{2}}\right\rangle F_{t_{\epsilon}}^{T}\right\| .
$$

Equations (11.10), (11.11) give (11.9). Therefore we have

$$
\begin{aligned}
\lim _{T \rightarrow \infty}\left\|\chi_{2}(H) \mathrm{e}^{-i t_{\epsilon} H} F_{t_{\epsilon}}^{T}\right\| & =\lim _{T \rightarrow \infty}\left\|\chi_{2}\left(H_{\leftarrow}\right) \mathrm{e}^{-i t_{\epsilon} H_{\leftarrow}} F_{t_{\epsilon}}^{T}\right\| \\
& =\lim _{T \rightarrow \infty}\left\|\chi_{2}\left(H_{\leftarrow}\right) F_{0}^{T}\right\|=\lim _{T \rightarrow \infty}\left\|\mathbf{1}_{[0, \infty)}\left(H_{\leftarrow}\right) F_{0}^{T}\right\| .
\end{aligned}
$$

In order to replace $\chi_{2}\left(H_{\leftarrow}\right)$ by $\mathbf{1}_{[0, \infty)}\left(H_{\leftarrow}\right)$ we use the same arguments as before.

\subsection{The term near the horizon}

We first compute the radiation explicitly for the asymptotic dynamics:

Lemma 11.4. - We have

$$
\lim _{T \rightarrow \infty}\left\|\mathbf{1}_{[0, \infty)}\left(H_{\leftarrow}\right) F_{0}^{T}\right\|^{2}=\left\langle\tilde{f}, \mathrm{e}^{\left(2 \pi / \kappa_{+}\right) H_{\leftarrow}}\left(1+\mathrm{e}^{\left(2 \pi / \kappa_{+}\right) H_{\leftarrow}}\right)^{-1} \tilde{f}\right\rangle .
$$

Proof. - The proof is analogous to the proof of [6, Lemma VI.6]. We repeat it here for the convenience of the reader. Let $\mathcal{F}$ be the Fourier transform with respect to $\widehat{r}$. We have

$$
\begin{aligned}
& \left\|\mathbf{1}_{[0, \infty)}\left(H_{\leftarrow}\right) F_{0}^{T}\right\|^{2}=\int_{S^{2}} \int_{0}^{\infty}\left|\mathcal{F}\left(F_{0}^{T}\right)(\xi)\right|^{2} \mathrm{~d} \xi \mathrm{d} \omega \\
& \quad=\sum_{j=1}^{2} \lim _{\epsilon \rightarrow 0^{+}} \int_{S^{2}} \widehat{A}(\theta) \kappa_{+} \int_{0}^{\infty}\left|\int_{\mathbb{R}} \mathrm{e}^{i(\widehat{A}(\theta)+i \epsilon) \xi \mathrm{e}^{\kappa+y}} \mathrm{e}^{\frac{1}{2} \kappa_{+} y} \tilde{f}_{j} \mathrm{~d} y\right|^{2} d \xi \mathrm{d} \omega
\end{aligned}
$$




$$
\begin{aligned}
&=\sum_{j=1}^{2} \lim _{\epsilon \rightarrow 0^{+}} \int_{S^{2}} \frac{1}{2} \widehat{A}(\theta) \kappa_{+} \\
& \times \int_{\mathbb{R} \times \mathbb{R}} \frac{\tilde{f}_{j}\left(y_{1}\right) \overline{\widetilde{f}}_{j}\left(y_{2}\right) \mathrm{d} y_{1} \mathrm{~d} y_{2}}{\epsilon \cosh \left(\frac{1}{2} \kappa_{+}\left(y_{1}-y_{2}\right)\right)-i \widehat{A}(\theta) \sinh \left(\frac{1}{2} \kappa_{+}\left(y_{1}-y_{2}\right)\right)} \\
&=\sum_{j=1}^{2} \lim _{\epsilon \rightarrow 0^{+}} \int_{S^{2}} \frac{\widehat{A}(\theta) \kappa_{+}}{4 \pi} \int_{\mathbb{R}}\left|\mathcal{F}\left(\tilde{f}_{j}\right)(\xi, \omega)\right|^{2} \\
& \quad \times \mathcal{F}\left(\frac{1}{\epsilon \cosh \left(\frac{1}{2} \kappa_{+} x\right)-i \widehat{A}(\theta) \sinh \left(\frac{1}{2} \kappa_{+} x\right)}\right)(-\xi) \mathrm{d} \xi \mathrm{d} \omega .
\end{aligned}
$$

Now given $\epsilon \neq 0, \xi<0$ and $N, M>0$, we evaluate

$$
\oint \ell(x) \mathrm{d} x, \quad \ell(x):=\frac{\mathrm{e}^{-i x \xi}}{\epsilon \cosh \left(\frac{1}{2} \kappa_{+} x\right)-i \widehat{A}(\theta) \sinh \left(\frac{1}{2} \kappa_{+} x\right)},
$$

along the path

$$
\{-N \leq \Re x \leq N, \Im x=0, M\} \cup\{0 \leq \Im x \leq M, \Re x= \pm N\} .
$$

First we have

$$
\begin{aligned}
& \left|\int_{ \pm N}^{ \pm N+i M} \ell(x) \mathrm{d} x\right| \lesssim \mathrm{e}^{-\frac{1}{2} \kappa_{+} N} \int_{0}^{\infty} \mathrm{e}^{x \xi} \mathrm{d} x \longrightarrow 0, \quad N \rightarrow \infty \\
& \left|\int_{-N+i M}^{N+i M} \ell(x) \mathrm{d} x\right| \lesssim \mathrm{e}^{M \xi} \int_{-\infty}^{\infty} \mathrm{e}^{-\frac{1}{2} \kappa_{+}|x|} \mathrm{d} x \longrightarrow 0, \quad M \rightarrow \infty
\end{aligned}
$$

We deduce that

$$
\int_{-\infty}^{\infty} \ell(x) \mathrm{d} x=2 i \pi \sum_{n=1}^{\infty} \rho_{n}(\epsilon)
$$

where $\rho_{n}(\epsilon)$ are the residues of $\ell(x)$ at the poles $z_{n}(\epsilon) \in\{z \in \mathbb{C}$; $₹>0\}$. We easily check that

$$
z_{n}(\epsilon)=\frac{2 i}{\kappa_{+}}\left(n \pi-\arctan \left(\frac{\epsilon}{\widehat{A}(\theta)}\right)\right), \quad \sup _{1 \leq n}\left|\rho_{n}(\epsilon)-\frac{2 i}{\widehat{A}(\theta) \kappa_{+}}(-1)^{n} \mathrm{e}^{\left(2 n \pi / \kappa_{+}\right) \xi}\right| \lesssim \epsilon,
$$

hence we get that for $\xi<0$ we have

$$
\left|\mathcal{F}\left(\frac{1}{\epsilon \cosh \left(\frac{1}{2} \kappa_{+} x\right)-i \widehat{A}(\theta) \sinh \left(\frac{1}{2} \kappa_{+} x\right)}\right)(\xi)-\frac{4 \pi}{\widehat{A}(\theta) \kappa_{+}} \mathrm{e}^{\left(2 \pi / \kappa_{+}\right) \xi}\left(1+\mathrm{e}^{\frac{2 \pi}{\kappa_{+}} \xi}\right)^{-1}\right| \lesssim \epsilon .
$$


In the same manner, for $\xi>0$, choosing $M<0$ and considering the poles $z_{n}(\epsilon) \epsilon$ $\{z \in \mathbb{C} ; \Im z<0\}$ we obtain

$$
\begin{gathered}
\int_{-\infty}^{\infty} \ell(x) \mathrm{d} x=2 i \pi \sum_{n=0}^{-\infty} \rho_{n}(\epsilon), \\
\sup _{n \leq 0}\left|\rho_{n}(\epsilon)-\frac{2 i}{\widehat{A}(\theta) \kappa_{+}}(-1)^{n} \mathrm{e}^{-\frac{2 n \pi}{\kappa_{+}} \xi}\right| \lesssim \epsilon, \\
\left|\mathcal{F}\left(\frac{1}{\epsilon \cosh \left(\frac{1}{2} \kappa_{+} x\right)-i \widehat{A}(\theta) \sinh \left(\frac{1}{2} \kappa_{+} x\right)}\right)(\xi)-\frac{4 \pi}{\widehat{A}(\theta) \kappa_{+}}\left(1+\mathrm{e}^{-\frac{2 \pi}{\kappa_{+}} \xi}\right)^{-1}\right| \lesssim \epsilon .
\end{gathered}
$$

Eventually we conclude that

$$
\mathcal{F}\left(\frac{1}{0-i \widehat{A}(\theta) \sinh \left(\frac{1}{2} \kappa_{+} x\right)}\right)(\xi)=\frac{4 \pi}{\kappa_{+}} \mathrm{e}^{\frac{2 \pi}{\kappa_{+}} \xi}\left(1+\mathrm{e}^{\frac{2 \pi}{\kappa_{+}} \xi}\right)^{-1},
$$

and

$$
\begin{aligned}
\left\|\mathbf{1}_{[0, \infty)}\left(H_{\leftarrow}\right) F_{0}^{T}\right\|^{2} & =\int_{S^{2}} \int_{\mathbb{R}} \mathrm{e}^{-\frac{2 \pi}{\kappa_{+}} \xi}\left(1+\mathrm{e}^{-\frac{2 \pi}{\kappa_{+}} \xi}\right)^{-1}\left|\mathcal{F}\left(\tilde{f}_{j}\right)(\xi, \omega)\right|^{2} d \xi \mathrm{d} \omega \\
& =\left\langle\tilde{f}, \mathrm{e}^{\frac{2 \pi}{\kappa_{+}} H_{\leftarrow}}\left(1+\mathrm{e}^{\frac{2 \pi}{\kappa_{+}} H_{\leftarrow}}\right)^{-1} \tilde{f}\right\rangle .
\end{aligned}
$$

Proposition 11.1. - We have

$$
\lim _{T \rightarrow \infty}\left\|\mathbf{1}_{[\eta, \infty)}\left(H_{0}\right) \mathcal{\partial}(0, T) f\right\|_{0}^{2}=\left\langle\Omega_{\leftarrow}^{-} f, \mathrm{e}^{\frac{2 \pi}{\kappa+} H \leftarrow}\left(1+\mathrm{e}^{\frac{2 \pi}{\kappa+} H \leftarrow}\right)^{-1} \Omega_{\leftarrow}^{-} f\right\rangle .
$$

Proof. - For $\epsilon>0$ given we estimate

$$
\begin{aligned}
& \left|\left\|\mathbf{1}_{[\eta, \infty)}\left(H_{0}\right) \mathcal{J U}(0, T) f\right\|_{0}^{2}-\left\langle\Omega_{\leftarrow}^{-} f, \mathrm{e}^{\sigma H \leftarrow}\left(1+\mathrm{e}^{\sigma H_{\leftarrow}}\right)^{-1} \Omega_{\leftarrow}^{-} f\right\rangle\right| \\
& \lesssim\left\|\left(\Omega_{\leftarrow}^{-} f\right)_{R}^{N}-\Omega_{\leftarrow}^{-} f\right\| \cdot\left\|\Omega_{\leftarrow}^{-} f\right\| \\
& \quad+\left|\left\|\mathbf{1}_{[\eta, \infty)}\left(H_{0}\right) \mathcal{\partial} U(0, T) f\right\|_{0}^{2}-\left\|\mathbf{1}_{[\eta, \infty)}\left(H_{0}\right) \widetilde{\phi}_{\delta} \mathrm{e}^{i t_{\epsilon} H} F_{t_{\epsilon}}^{T}\right\|^{2}\right| \\
& \quad+\left|\left\|\mathbf{1}_{[\eta, \infty)}\left(H_{0}\right) \widetilde{\phi}_{\delta} \mathrm{e}^{i t_{\epsilon} H} F_{t_{\epsilon}}^{T}\right\|^{2}-\left\|\mathbf{1}_{[0, \infty)}\left(H_{\leftarrow}\right) F_{0}^{T}\right\|^{2}\right| \\
& \quad+\mid\left\|\mathbf{1}_{[0, \infty)}\left(H_{\leftarrow}\right) F_{0}^{T}\right\|^{2}-\left\langle\widetilde{f}, \mathrm{e}^{\left.\sigma H \leftarrow\left(1+\mathrm{e}^{\sigma H \leftarrow}\right)^{-1} \tilde{f}\right\rangle \mid}\right. \\
& =: I_{1}+I_{2}+I_{3}+I_{4} .
\end{aligned}
$$

Here $\delta=\delta\left(t_{\epsilon}\right)$ as in Proposition 10.1. We first choose $R_{0}, N_{0}>0$ such that

$$
\forall N \geq N_{0}, R \geq R_{0}, \quad I_{1}<\frac{1}{4} \epsilon .
$$

Using Proposition 10.1 we can fix $N \geq N_{0}, R \geq R_{0}, t_{\epsilon}>0, \delta=\delta\left(t_{\epsilon}\right)>0$ and $T_{0}>0$ such that

$$
\forall T \geq T_{0}, \quad I_{2}<\frac{1}{4} \epsilon .
$$

By choosing $T_{0}$ possibly larger we have:

$$
\forall T \geq T_{0}, \quad I_{3}<\frac{1}{4} \epsilon .
$$


Here we have used Lemma 11.3 and (11.6). Note that the same $t_{\epsilon}$ can be chosen for the estimate of $I_{2}, I_{3}$. Indeed in both cases we use Lemma 10.7. The parameters $N, R, t_{\epsilon}, \delta$ being fixed in this way we conclude by noting that $I_{4} \rightarrow 0, T \rightarrow \infty$.

\subsection{Proof of Theorem 8.2}

We start with the identity

$$
\begin{aligned}
\left\|\mathbf{1}_{[\eta, \infty)}\left(H_{0}\right) U(0, T) f\right\|_{0}^{2} & \\
= & \left\|\mathbf{1}_{[\eta, \infty)}\left(H_{0}\right) \mathcal{J} U(0, T) f\right\|_{0}^{2}+\left\|\mathbf{1}_{[\eta, \infty)}\left(H_{0}\right)(1-\mathcal{J}) U(0, T) f\right\|_{0}^{2} \\
& \quad+2 \Re\left\langle\mathbf{1}_{[\eta, \infty)}\left(H_{0}\right)(1-\mathcal{\jmath}) U(0, T) f, \mathbf{1}_{[\eta, \infty)}\left(H_{0}\right) \mathcal{\partial} U(0, T) f\right\rangle .
\end{aligned}
$$

In order to prove that

$$
\left\langle\mathbf{1}_{[\eta, \infty)}\left(H_{0}\right)(1-\mathcal{J}) U(0, T) f, \mathbf{1}_{[\eta, \infty)}\left(H_{0}\right) \mathcal{U} U(0, T) f\right\rangle \longrightarrow 0, \quad T \rightarrow \infty
$$

it is enough, by the results of the preceding chapters, to prove

$$
\left\langle\mathbf{1}_{[\eta, \infty)}\left(H_{0}\right)(1-\mathcal{J}) U(0, T) f, \mathbf{1}_{[\eta, \infty)}\left(H_{0}\right) \widetilde{\phi}_{\delta} \mathrm{e}^{-i t_{\epsilon} H} F_{t_{\epsilon}}^{T}\right\rangle \longrightarrow 0, \quad T \rightarrow \infty .
$$

Note that

Indeed

$$
\mathbf{1}_{[\eta, \infty)}\left(H_{0}\right)\left(H_{0}+i\right)^{-1} \widetilde{\phi}_{\delta} \mathrm{e}^{-i t_{\epsilon} H} F_{t_{\epsilon}}^{T} \longrightarrow 0, \quad T \rightarrow \infty
$$

$$
\begin{aligned}
& \mathbf{1}_{[\eta, \infty)}\left(H_{0}\right)\left(H_{0}+i\right)^{-1} \widetilde{\phi}_{\delta} \mathrm{e}^{-i t_{\epsilon} H} F_{t_{\epsilon}}^{T} \\
& \quad=\mathbf{1}_{[\eta, \infty)}\left(H_{0}\right)\left(H_{0}+i\right)^{-1} \widetilde{\phi}_{\delta}(H+i) \mathrm{e}^{-i t_{\epsilon} H}(H+i)^{-1} F_{t_{\epsilon}}^{T} \longrightarrow 0, \quad T \rightarrow \infty .
\end{aligned}
$$

It is therefore sufficient to show that for all $f \in \mathcal{H}^{1}$ the following limit exists:

$$
\lim _{T \rightarrow \infty}\left(H_{0}+i\right) \mathrm{e}^{i T H_{0}}(1-\mathcal{J}) \mathrm{e}^{-i T H} f .
$$

But,

$$
\begin{aligned}
& \left(H_{0}+i\right) \mathrm{e}^{i T H_{0}}(1-\mathcal{\jmath}) \mathrm{e}^{-i T H} f \\
& =-\mathrm{e}^{-i T H_{0}}\left[H, \mathcal{\jmath} \mathrm{e}^{-i T H} f+\mathrm{e}^{i T H_{0}}(1-\mathcal{\jmath}) \mathrm{e}^{-i T H}(H+i) f\right. \\
& \quad=-\mathrm{e}^{i T H_{0}} \Gamma^{1} \mathcal{J}^{\prime} \mathrm{e}^{-i T H} f+\mathrm{e}^{i T H_{0}}(1-\mathcal{\jmath}) \mathrm{e}^{-i T H}(H+i) f .
\end{aligned}
$$

The first term goes to zero because $\sigma_{s c}(H)=\varnothing$ and the second term possesses a limit by Lemma 6.5. By the same lemma we find

$$
\begin{aligned}
\lim _{T \rightarrow \infty}\left\|\mathbf{1}_{[\eta, \infty)}\left(H_{0}\right)(1-\mathcal{J}) U(0, T) f\right\| & =\left\|\mathbf{1}_{[\eta, \infty)}\left(H_{0}\right) W_{0}^{-} f\right\|=\left\|W_{0}^{-} \mathbf{1}_{[\eta, \infty)}(H) f\right\| \\
& =\left\|\mathbf{1}_{\mathbb{R}^{-}}\left(P^{-}\right) \mathbf{1}_{[\eta, \infty)}(H) f\right\| \\
& =\left\|\mathbf{1}_{[\eta, \infty)}(H) \mathbf{1}_{\mathbb{R}^{-}}\left(P^{-}\right) f\right\| .
\end{aligned}
$$

This concludes the proof of the theorem. 


\section{APPENDIX A}

\section{PROOF OF PROPOSITION 8.2}

We will work with the $\left(r_{*}, \omega\right)$ coordinate system. For technical reasons we need to fix the angular momentum $D_{\varphi}=n$. Let $\mathrm{d} \widetilde{\omega}=\sin \theta \mathrm{d} \theta$,

$$
\begin{gathered}
\widetilde{\mathcal{H}}^{n}=\left\{u=\mathrm{e}^{i n \varphi} v ; v \in\left(L^{2}\left(\mathbb{R} \times[0, \pi], \mathrm{d} r_{*} \mathrm{~d} \widetilde{\omega}\right)\right)^{4}\right\}, \\
\widetilde{\mathcal{H}}_{t}^{n}=\left\{u=\mathrm{e}^{i n \varphi} v ; v \in\left(L^{2}\left(\widetilde{\Sigma}_{t}^{\mathrm{col}, \varphi}, \mathrm{d} r_{*} \mathrm{~d} \widetilde{\omega}\right)\right)^{4}\right\}, \\
\widetilde{\Sigma}_{t}^{\mathrm{col}, \varphi}=\left\{\left(r_{*}, \theta\right) \in \mathbb{R} \times[0, \pi] ; r_{*} \geq z(t, \theta)\right\} .
\end{gathered}
$$

We define:

$$
\begin{aligned}
& \widetilde{\not D}^{\nu, n}:=h \widetilde{D}_{\mathfrak{s}}^{\nu, n} h+V^{n}, \\
& \widetilde{\mathbb{D}}_{\mathfrak{s}}^{\nu, n}:=\Gamma^{1} D_{r_{*}}+a_{0} \Gamma^{2}\left(D_{\theta}+\frac{1}{2} \cot \theta\right)+a_{0} \Gamma^{3} \frac{n}{\sin \theta}+b_{0} \Gamma^{\nu}+c^{n}, \\
& \widetilde{\mathcal{H}}^{n 1}=D\left(\widetilde{D}^{\nu, n}\right)=\left\{u \in \widetilde{\mathcal{H}}^{n} ; \widetilde{D}^{\nu, n} u \in \widetilde{\mathcal{H}}^{n}\right\} .
\end{aligned}
$$

Recall that the singularity $\sin \theta=0$ appearing in the expression for $\widetilde{D}_{\mathfrak{s}}^{\nu, n}$ is a coordinate singularity. The operator $\widetilde{D}_{t}^{\nu, n}$ is the operator acting on $\widetilde{\mathcal{H}}_{t}^{n}$ with formal expression $\widetilde{D D}^{\nu, n}$ and domain

$$
\begin{aligned}
\widetilde{\mathcal{H}}_{t}^{n 1} & :=D\left(\widetilde{\mathbb{D}}_{t}^{\nu, n}\right) \\
& =\left\{u \in \widetilde{\mathscr{H}}_{t}^{n} ; \widetilde{\mathbb{D}}_{t}^{\nu, n} u \in \widetilde{\mathscr{H}}_{t}^{n}, \sum_{\mu \in\left\{t, r_{*}, \theta, \varphi\right\}} \mathcal{N}_{\mu} \gamma^{\mu} u(z(t, \theta), \theta)=-i u(z(t, \theta), \theta)\right\} .
\end{aligned}
$$

The extension $[.]_{H}^{*}$ is defined in an analogous way to Section 4.5 as an extension from $\widetilde{\mathscr{H}}_{t}^{n 1}$ to $\widetilde{\mathscr{H}}^{n 1}$. We consider the problem

$$
\left\{\begin{array}{l}
\partial_{t} \Psi=i \widetilde{D}_{t}^{\nu, n} \Psi, \quad r_{*}>z(t, \theta) \\
\sum_{\mu \in\left\{t, r_{*}, \theta, \varphi\right\}} \mathcal{N}_{\mu} \gamma^{\mu} \Psi(t, z(t, \theta), \theta)=-i \Psi(t, z(t, \theta), \theta), \\
\Psi(t=s, .)=\Psi_{s}(.) \in D\left(\widetilde{D}_{s}^{\nu, n}\right) .
\end{array}\right.
$$

Proposition 8.2 follows from: 
Proposition A.1. - Let $\Psi_{s} \in D\left(\widetilde{\not D}_{s}^{\nu, n}\right)$. Then there exists a unique solution

$$
[\Psi(.)]_{H}^{*}=\left[\widetilde{U}^{\nu, n}(., s) \Psi_{s}\right]_{H}^{*} \in C^{1}\left(\mathbb{R} ; \tilde{\mathscr{H}}^{n}\right) \cap C\left(\mathbb{R} ; \tilde{\mathscr{H}}^{n 1}\right)
$$

of (A.3) such that for all $t \in \mathbb{R} \Psi(t) \in D\left(\widetilde{D}_{t}^{\nu, n}\right)$. Furthermore we have

$$
\|\Psi(t)\|=\left\|\Psi_{s}\right\|
$$

and $\widetilde{U}^{\nu, n}(t, s)$ possesses an extension to an isometric and strongly continuous propagator from $\widetilde{\mathcal{H}}_{s}^{n}$ to $\widetilde{\mathcal{H}}_{t}^{n}$ such that for all $\Psi_{s} \in D\left(\widetilde{D}_{s}^{\nu, n}\right)$ we have

$$
\frac{\mathrm{d}}{\mathrm{d} t} \widetilde{U}^{\nu, n}(t, s) \Psi_{s}=i \widetilde{\not D}_{t}^{\nu, n} \widetilde{U}^{\nu, n}(t, s) \Psi_{s}
$$

Note that in Proposition 8.2, there is an additional statement about the finite propagation speed which follows from ( $\Psi$ is supposed to be a solution of $(8.14)$ )

$$
\begin{aligned}
\frac{\mathrm{d}}{\mathrm{d} t} \int_{S^{2}} \int_{R+|t-s|}^{\infty}|\Psi(t, \widehat{r}, \omega)|^{2} \mathrm{~d} \widehat{r} \mathrm{~d} \omega & =2 \mathbf{1}_{\mathbb{R}^{+}}(s-t) \int_{S^{2}}\left(\left|\Psi_{2}\right|^{2}+\left|\Psi_{3}\right|^{2}\right) \mathrm{d} \omega \\
& -2 \mathbf{1}_{\mathbb{R}^{+}}(t-s) \int_{S^{2}}\left(\left|\Psi_{1}\right|^{2}+\left|\Psi_{4}\right|^{2}\right) \mathrm{d} \omega .
\end{aligned}
$$

Proof of Proposition A.1. - We will drop the indices $\nu, n$ in what follows.

$\triangleright$ Let us first show uniqueness. If $\Psi$ is solution of (A.3), then we have

$$
\begin{aligned}
\frac{\mathrm{d}}{\mathrm{d} t} \int_{0}^{\pi} \int_{z(t, \theta)}^{\infty}|\Psi|^{2} \mathrm{~d} r_{*} \mathrm{~d} \widetilde{\omega} & \\
& =-\int_{0}^{\pi} \dot{z}(t, \theta)|\Psi|^{2}(z(t, \theta), \theta) \mathrm{d} \omega+2 \Re\left\langle i \widetilde{D}_{t} \Psi, \Psi\right\rangle \\
& =2 \Re\left\langle i \check{D}_{t} \Psi, \Psi\right\rangle=0 .
\end{aligned}
$$

Here $\check{\not}_{t}:=\dot{z} D_{r_{*}}+\widetilde{\mathbb{D}}_{t}$ is selfadjoint with domain $\widetilde{\mathcal{H}}_{t}^{n 1}$ (see Lemma 4.2).

$\triangleright$ Let us now prove existence. We introduce the operators

$$
\begin{gathered}
R(t)=\left(N^{2}+Z_{1}^{2}-Z_{2}^{2}\right)^{-\frac{1}{2}}\left(\begin{array}{rrrr}
N & 0 & -Z_{1} & -Z_{2} \\
0 & N & -Z_{2} & -Z_{1} \\
Z_{1} & -Z_{2} & N & 0 \\
-Z_{2} & Z_{1} & 0 & N
\end{array}\right), \\
N=w^{-1}, \quad Z_{1}=h^{2}+\dot{z}, \quad Z_{2}=a_{0} h^{2} \partial_{\theta} z, \\
\mathcal{J}: \mathscr{H}_{0} \ni f \longmapsto \\
\mathcal{J}(t) f \in \mathcal{H}_{t}, \quad[\mathcal{T}(t) f]\left(r_{*}, \omega\right)=f\left(r_{*}-z(t, \theta)+z(0, \theta), \omega\right) .
\end{gathered}
$$


We remark that

$$
R^{-1}(t)=\left(N^{2}+Z_{1}^{2}-Z_{2}^{2}\right)^{-\frac{1}{2}}\left(\begin{array}{cccc}
N & 0 & Z_{1} & Z_{2} \\
0 & N & Z_{2} & Z_{1} \\
-Z_{1} & Z_{2} & N & 0 \\
Z_{2} & -Z_{1} & 0 & N
\end{array}\right) .
$$

Furthermore we notice that

$$
\mathcal{J} \in C^{1}\left(\mathbb{R}_{t} ; \mathscr{L}\left(\left(C_{0}^{1}\left(\mathbb{R}_{r_{*}} \times[0, \pi]\right)\right)^{4},\left(C_{0}^{0}\left(\mathbb{R}_{r_{*}} \times[0, \pi]\right)\right)^{4}\right), \quad \dot{\mathcal{J}}(t)=-\dot{z}(t, \theta) \mathcal{J}(t) \partial_{r_{*}} .\right.
$$

Then $u$ is a solution of our problem iff $w(t)=R^{-1}(t) \mathcal{T}^{-1}(t) u$ is solution of

$$
\left\{\begin{array}{l}
\partial_{t} w=i A(t) w, \quad r_{*}>z(0, \theta), \\
w_{2}(t, z(0, \theta), \omega)=w_{3}(t, z(0, \theta), \omega)=0,
\end{array}\right.
$$

where $A(t)=R^{-1}(t) \mathcal{T}^{-1}(t)\left(\not D+\dot{z} D_{r_{*}}\right) R(t) \mathcal{J}(t)-R^{-1}(t) \dot{R}(t)$. We first need to analyze $A(t)$ for fixed $t$. We put

$$
D(A(t))=\left\{u \in \widetilde{\mathscr{H}}_{0} ; A(t) u \in \widetilde{\mathscr{H}}_{0}, u_{2}(z(0, \theta), \omega)=u_{3}(z(0, \theta), \omega)=0\right\} .
$$

We equip $D(A(t))$ with the graph norm of $A(t)$. The operator $(A(t), D(A(t))$ is selfadjoint (see Lemma 4.2). Let

$$
\mathrm{d}_{\theta}:=D_{\theta}+\frac{\cot \theta}{2 i}
$$

We first note that the domain is independent of $t$ :

$$
\begin{aligned}
& \forall t, D(A(t))=D \\
& \quad:=\left\{u \in \widetilde{\mathcal{H}}_{0} ; D_{r_{*}} u \in \widetilde{\mathcal{H}}_{0}, \mathrm{~d}_{\theta} u \in \widetilde{\mathcal{H}}_{0}, u_{2}(z(0, \theta), \theta)=u_{3}(z(0, \theta), \theta)=0\right\}
\end{aligned}
$$

and the graph norm of $A(t)$ is equivalent to the norm

$$
\|u\|_{D}=\left\|D_{r_{*}} u\right\|+\left\|a_{0} \mathrm{~d}_{\theta} u\right\|+\|u\| .
$$

This follows from the estimates

$$
\begin{aligned}
& \forall u \in D(A(t)), \quad\left\|D_{r_{*}} u\right\| \lesssim\|A(t) u\|+\|u\|, \\
& \forall u \in D(A(t)), \quad\left\|a_{0} \mathrm{~d}_{\theta} u\right\| \lesssim\|A(t) u\|+\|u\| .
\end{aligned}
$$

To show (A.5) and (A.6) we introduce the operator $\widetilde{D}^{t}$ which is obtained from $\widetilde{D}$ by evaluating all functions in the definition of $\widetilde{D}$ at $\left(r_{*}+z(t, \theta)-z(0, \theta), \theta\right)$. Then we have

$$
A(t)=R^{-1}(t)\left(\widetilde{D D}^{t}+\dot{z} D_{r_{*}}\right) R(t)-R^{-1}(t) \dot{R}(t) .
$$

For $u$ regular enough we estimate

$$
\begin{aligned}
\|A(t) u\| & \gtrsim\left\|\widetilde{D}^{t} R(t) u\right\|-\max _{\theta}|\dot{z}(t, \theta)| \cdot\left\|D_{r_{*}} R(t) u\right\|-C\|u\| \\
& \geq\left\|\widetilde{D}^{t} R(t) u\right\|-(1-\delta)\left\|D_{r_{*}} R(t) u\right\|-C\|u\|
\end{aligned}
$$


for some $0<\delta<1$. We henceforth drop the subscript $t$. Let

$$
v:=R(t) u \text {. }
$$

The first term on the R.H.S. in the above inequality can be estimated in the following way:

$$
\left\|\widetilde{D D}^{t} v\right\|^{2} \geq\left\|h^{2} D_{r_{*}} v\right\|^{2}+\left\|h^{2} a_{0}\left(D_{\theta}+\frac{1}{2} \cot \theta\right) v\right\|^{2}+2 \Re I-C\|v\|^{2}
$$

with

$$
I=\int_{0}^{\pi} \int_{z(t, \theta)}^{\infty}\left\langle h a_{0} \Gamma^{2}\left(D_{\theta}+\frac{1}{2} \cot \theta\right) h v, h \Gamma^{1} D_{r_{*}} h v\right\rangle \mathrm{d} r_{*} \mathrm{~d} \widetilde{\omega} .
$$

We have:

$$
\begin{gathered}
2 I=-\int_{0}^{\pi}\left\langle h a_{0} \Gamma^{2}\left(\partial_{\theta}+\frac{1}{2} \cot \theta\right) h v, \Gamma^{1} h^{2} v\right\rangle(z(t, \theta), \theta) \mathrm{d} \widetilde{\omega} \\
-\int_{0}^{\pi} \int_{z(t, \theta)}^{\infty}\left\langle\Gamma^{1} \partial_{r_{*}} h^{2} a_{0} \Gamma^{2}\left(\partial_{\theta}+\frac{1}{2} \cot \theta\right) h v, h v\right\rangle \mathrm{d} r_{*} \mathrm{~d} \widetilde{\omega} \\
\quad+\int_{0}^{\pi}\left(\partial_{\theta} z\right)\left\langle h v, h a_{0} \Gamma^{2} h \Gamma^{1} \partial_{r_{*}} h v\right\rangle(z(t, \theta), \theta) \mathrm{d} \widetilde{\omega} \\
\quad-\int_{0}^{\pi} \int_{z(t, \theta)}^{\infty}\left\langle h v, a_{0} \Gamma^{2}\left(\partial_{\theta}+\frac{1}{2} \cot \theta\right) h^{2} \Gamma^{1} \partial_{r_{*}} h v\right\rangle \mathrm{d} r_{*} \mathrm{~d} \widetilde{\omega} .
\end{gathered}
$$

Therefore

$$
\begin{aligned}
2 \Re I=-\Re \int_{0}^{\pi}\left\langle h a_{0} \Gamma^{2}\left(\partial_{\theta}+\frac{1}{2} \cot \theta\right) h v, \Gamma^{1} h^{2} v\right\rangle(z(t, \theta), \theta) \mathrm{d} \widetilde{\omega} \\
+\Re \int_{0}^{\pi}\left(\partial_{\theta} z\right)\left\langle h v, h^{2} a_{0} \Gamma^{2} \Gamma^{1} \partial_{r_{*}} h v\right\rangle(z(t, \theta), \theta) \mathrm{d} \widetilde{\omega} \\
\quad-2 \Re \int_{0}^{\pi} \int_{z(t, \theta)}^{\infty}\left\langle\left\{h \Gamma^{1} D_{r_{*}} h, h a_{0} \Gamma^{2}\left(D_{\theta}+\frac{1}{2 i} \cot \theta\right) h\right\} v, h v\right\rangle \mathrm{d} r_{*} \mathrm{~d} \widetilde{\omega} \\
=: B+A C .
\end{aligned}
$$

We first estimate the boundary term. We have

$$
B=\Re \int_{0}^{\pi}\left\langle h a_{0} \Gamma^{2} h v, \Gamma^{1} \tilde{\partial}_{\theta}((h v)(z(t, \theta), \theta))\right\rangle(z(t, \theta), \theta) \mathrm{d} \widetilde{\omega},
$$

where $\tilde{\partial}_{\theta}=\partial_{\theta}+\frac{1}{2} \cot \theta$. Recalling that

$$
h v(z(t, \theta), \theta)=\left(\tilde{N} h\left(\begin{array}{c}
N u_{1}-Z_{2} u_{4} \\
-Z_{1} u_{4} \\
Z_{1} u_{1} \\
-Z_{2} u_{1}+N u_{4}
\end{array}\right)\right)(z(t, \theta), \theta)
$$


with $\tilde{N}=\left(N^{2}+Z_{1}^{2}-Z_{2}^{2}\right)^{-\frac{1}{2}}$ we find

$$
\begin{aligned}
B \geq- & C\|u\|_{\left(L^{2}([0, \pi])^{4}\right.} \\
& +\int_{0}^{\pi} \tilde{N}^{2}\left\langle h\left(\begin{array}{c}
N u_{1}-Z_{2} u_{4} \\
-Z_{1} u_{4} \\
Z_{1} u_{1} \\
-Z_{2} u_{1}+N u_{4}
\end{array}\right), h^{3} a_{0} \Gamma^{2} \Gamma^{1}\left(\begin{array}{c}
N \tilde{\partial}_{\theta}\left(u_{1}\right)-Z_{2} \tilde{\partial}_{\theta}\left(u_{4}\right) \\
-Z_{1} \tilde{\partial}_{\theta}\left(u_{4}\right) \\
Z_{1} \tilde{\partial}_{\theta}\left(u_{1}\right) \\
-Z_{2} \tilde{\partial}_{\theta}\left(u_{1}\right)+N \tilde{\partial}_{\theta}\left(u_{4}\right)
\end{array}\right)\right\rangle \sin \theta \mathrm{d} \theta .
\end{aligned}
$$

Here $\tilde{\partial}_{\theta}\left(u_{j}\right)$ stands for $\tilde{\partial}_{\theta}\left(u_{j}(z(t, \theta), \theta)\right)$. The term under the second integral in the above inequality equals

$$
\begin{gathered}
\tilde{N}^{2}\left\langle h^{4} a_{0}\left(\begin{array}{c}
N u_{1}-Z_{2} u_{4} \\
-Z_{1} u_{4} \\
Z_{1} u_{1} \\
-Z_{2} u_{1}+N u_{4}
\end{array}\right),\left(\begin{array}{c}
Z_{1} \tilde{\partial}_{\theta}\left(u_{4}\right) \\
N \tilde{\partial}_{\theta}\left(u_{1}\right)-Z_{2} \tilde{\partial}_{\theta}\left(u_{4}\right) \\
Z_{2} \tilde{\partial}_{\theta}\left(u_{1}\right)-N \tilde{\partial}_{\theta}\left(u_{4}\right) \\
Z_{1} \tilde{\partial}_{\theta}\left(u_{1}\right)
\end{array}\right)\right\rangle \\
=\tilde{N}^{2}\left(h ^ { 4 } a _ { 0 } \left(N Z_{1} u_{1} \tilde{\partial}_{\theta}\left(\bar{u}_{4}\right)-Z_{2} u_{4} Z_{1} \tilde{\partial}_{\theta}\left(\bar{u}_{4}\right)-Z_{1} u_{4} N \tilde{\partial}_{\theta}\left(\bar{u}_{1}\right)+Z_{1} Z_{2} u_{4} \tilde{\partial}_{\theta}\left(\bar{u}_{4}\right)\right.\right. \\
\left.\left.\quad+Z_{1} Z_{2} u_{1} \tilde{\partial}_{\theta}\left(\bar{u}_{1}\right)-Z_{1} u_{1} N \tilde{\partial}_{\theta}\left(\bar{u}_{4}\right)-Z_{2} Z_{1} u_{1} \tilde{\partial}_{\theta}\left(\bar{u}_{1}\right)+N Z_{1} u_{4} \tilde{\partial}_{\theta}\left(\bar{u}_{1}\right)\right)\right)=0 .
\end{gathered}
$$

Thus $B \geq-C\|u(z(t, \theta), \theta)\|_{\left(L^{2}([0, \pi])\right)^{4}}$. By the usual trace theorems we find

$$
B \geq-C\|u\|_{H^{\frac{1}{2}}\left(\Sigma_{t}^{\mathrm{col}, \varphi}\right)} \geq-\epsilon\left(\left\|D_{r_{*}} R(t) u\right\|+\left\|a_{0} \mathrm{~d}_{\theta} R(t) u\right\|\right)-C_{\epsilon}\|u\| .
$$

Let us now consider the anticommutator

$$
\left\{h \Gamma^{1} D_{r_{*}} h, h a_{0} \Gamma^{2} \mathrm{~d}_{\theta} h\right\}=h\left(\left[D_{r_{*}}, h^{2} a_{0}\right] \Gamma^{1} \Gamma^{2} \mathrm{~d}_{\theta}+a_{0}\left[\mathrm{~d}_{\theta}, h^{2}\right] \Gamma^{2} \Gamma^{1} D_{r_{*}}\right) h .
$$

Thus

$$
A C \geq-\epsilon\left(\left\|a_{0} \mathrm{~d}_{\theta} v\right\|+\left\|D_{r_{*}} v\right\|\right)-C_{\epsilon}\|v\|, \quad \epsilon>0 .
$$

Gathering everything together and using $h^{2} \geq 1$ we find

$$
\begin{aligned}
\|A(t) u\| \geq(1-\tilde{\epsilon}) & \left(\left\|D_{r_{*}} R(t) u\right\|+\left\|a_{0} \mathrm{~d}_{\theta} R(t) u\right\|\right) \\
& -(1-\delta)\left\|D_{r_{*}} R(t) u\right\|-C_{\epsilon}\|u\| \\
& \geq(\delta-\tilde{\epsilon})\left\|D_{r_{*}} u\right\|+(1-\epsilon)\left\|a_{0} \mathrm{~d}_{\theta} u\right\|-C_{\epsilon}\|u\|, \quad \tilde{\epsilon}<\delta .
\end{aligned}
$$

Recall that we have dropped the index $t$. But $a_{0} \lesssim a_{0}^{t} \lesssim a_{0}$. The above inequality proves (A.5), (A.6). The operators $(A(t), D)$ are selfadjoint and the family $\{(i A(t), D)\}_{t \in \mathbb{R}}$ is a stable family in the sense of [43, Definition 5.2.1]. We want to check that for $v \in D, t \mapsto A(t) v$ is continuously differentiable. We have

$$
\begin{aligned}
& \frac{A(t+\delta)-A(t)}{\delta} v \\
& =\frac{1}{\delta}\left(R^{-1}(t+\delta)\left(\widetilde{\mathbb{D}}^{t+\delta}+\dot{z} D_{r_{*}}\right) R(t+\delta)\right. \\
& \left.\quad-R^{-1}(t)\left(\widetilde{\not}^{t}+\dot{z} D_{r_{*}}\right) R(t)\right) v-\left(R^{-1}(t) \dot{R}(t)\right)^{\prime} v+o(\delta)
\end{aligned}
$$




$$
\begin{aligned}
& =\frac{R^{-1}(t+\delta)-R^{-1}(t)}{\delta}\left(\widetilde{\not D}^{t+\delta}+\dot{z} D_{r_{*}}\right) R(t+\delta) v \\
& +\frac{1}{\delta} R^{-1}(t)\left(\widetilde{\not D}^{t+\delta}+\dot{z} D_{r_{*}}\right) R(t+\delta) v \\
& \quad-\frac{1}{\delta} R^{-1}(t)\left(\widetilde{\not D}^{t}+\dot{z} D_{r_{*}}\right) R(t) v-\left(R^{-1}(t) \dot{R}(t)\right)^{\prime} v+o(\delta) \\
& =\frac{R^{-1}(t+\delta)-R^{-1}(t)}{\delta}\left(\widetilde{\not D}^{t+\delta}+\dot{z} D_{r_{*}}\right) R(t+\delta) v \\
& \quad+R^{-1}(t) \frac{\widetilde{D^{t+\delta}}-\widetilde{\not D}^{t}}{\delta} R(t+\delta) v \\
& \quad+R^{-1}(t)\left(\widetilde{\not D}^{t}+\dot{z} D_{r_{*}}\right) \frac{R(t+\delta)-R(t)}{\delta} v-\left(R^{-1}(t) \dot{R}(t)\right)^{\prime} v+o(\delta) \\
& =: I_{1}^{\delta}+I_{2}^{\delta}+I_{3}^{\delta}-\left(R^{-1}(t) \dot{R}(t)\right)^{\prime} v+o(\delta) .
\end{aligned}
$$

Here and henceforth a prime denotes a derivative with respect to $t$. Let

$$
\begin{aligned}
& I_{1}:=\left(R^{-1}(t)\right)^{\prime}\left(\widetilde{\mathbb{D}}^{t}+\dot{z} D_{r_{*}}\right) R(t) v, \\
& I_{2}:=R^{-1}(t)\left(\widetilde{\mathbb{D}}^{t}\right)^{\prime} R(t) v, \\
& I_{3}:=R^{-1}(t)\left(\widetilde{\mathbb{D}}^{t}+\dot{z} D_{r_{*}}\right)(R)^{\prime}(t) v .
\end{aligned}
$$

The operator $\left(\widetilde{D}^{t}\right)^{\prime}$ is defined by differentiating $\widetilde{D}^{t}$ formally with respect to $t$. Let us first consider the second term. We have

$$
\begin{aligned}
& \left\|\left(R^{-1}(t) \frac{\widetilde{D}^{t+\delta}-\widetilde{D}^{t}}{\delta} R(t+\delta)-R^{-1}(t)\left(\widetilde{\not D}^{t}\right)^{\prime} R(t)\right) v\right\| \\
& \leq\left\|R^{-1}(t)\left(\widetilde{D}^{t}\right)^{\prime}(R(t+\delta)-R(t)) v\right\| \\
& \quad+\left\|R^{-1}(t)\left(\frac{\widetilde{D}^{t+\delta}-\widetilde{\not D}^{t}}{\delta}-\left(\widetilde{\not D}^{t}\right)^{\prime}\right) R(t+\delta) v\right\| \\
& \leq\left\|D_{r_{*}}(R(t+\delta)-R(t)) v\right\|+\left\|a_{0}^{\prime} \mathrm{d}_{\theta}(R(t+\delta)-R(t)) v\right\| \\
& \quad+o(\delta)\left\|R^{-1}(t) \mathrm{d}_{\theta} R(t+\delta) v\right\|+o(\delta)\left\|R^{-1}(t) D_{r_{*}} R(t+\delta) v\right\| \\
& \leq o(\delta)\|v\|_{D} .
\end{aligned}
$$

Here we have used that $a_{0} \geq \epsilon$ on $[0, \infty) \times[0, \pi]$ as well as the estimates (A.5), (A.6). The other terms can be treated in a similar way. We find:

$$
\left\|I_{j}^{\delta}-I_{j}\right\| \leq o(\delta)\|v\|_{D}, \quad 1 \leq j \leq 3 .
$$

This shows that $t \mapsto A(t) v$ is continuously differentiable. We can therefore use [43, Theorem 5.4.8] to find a strongly continuous propagator $S(s, t)$ on $\tilde{\mathscr{H}}_{0}^{n}$ such that for $f \in D, S(t, s) f \in D$ is a strongly continuous differentiable map from $\mathbb{R}_{t} \times \mathbb{R}_{s}$ to $\tilde{\mathcal{H}}_{0}^{n}$ satisfying

$$
\frac{\mathrm{d}}{\mathrm{d} t} S(t, s) f=A(t) S(t, s) f, \quad \frac{\mathrm{d}}{\mathrm{d} s} S(t, s) f=-S(t, s) A(s) f .
$$


The propagator

$$
\widetilde{U}(t, s)=R(t) \mathcal{T}(t) S(t, s) R^{-1}(s) \mathcal{T}^{-1}(s)
$$

has the required properties. 



\section{APPENDIX B}

\section{PENROSE COMPACTIFICATION OF BLOCK $I$}

The Penrose compactification is usually obtained by a construction based on the PNG's. We present here an analogous construction based on the SNG's. In order to emphasize the analogy with the PNG construction we name the different coordinate systems that we introduce as in the PNG case. We will suppose $a>0$. The PNG construction is explained in detail in [42].

\section{B.1. Kerr-star and star-Kerr coordinates}

A part of the construction of this section can be found in [23] for the Kerr case. We will suppose $E=1$. Let $\widehat{r}$ be as in Chapter 3 . The star-Kerr coordinate system $\left({ }^{*} t, r,{ }^{*} \theta,{ }^{*} \varphi\right)$ is based on outgoing simple null geodesics. The new coordinates ${ }^{*} t,{ }^{*} \theta$ and $* \varphi$ are of the form

$$
{ }^{*} t=t-\widehat{r}(r, \theta), \quad{ }^{*} \varphi=\varphi-\Lambda(r), \quad{ }^{*} \theta=\theta^{\sharp}(r, \theta),
$$

where the function $\Lambda$ is required to satisfy

$$
\Lambda^{\prime}(r)=\frac{a\left(2 M r-Q^{2}\right)}{B(r) \Delta}, \quad B(r)=\left(r^{2}+a^{2}\right) k^{\prime}\left(r_{*}(r)\right) .
$$

The function $\theta^{\sharp}$ is defined in the following way. Let

$$
\alpha(r)=-\int_{r}^{\infty} \frac{a}{B(\nu)} \mathrm{d} \nu .
$$

For later simplicity we define

$$
F=1+\tanh (\alpha) \sin \theta^{\sharp}, \quad G=\tanh (\alpha)+\sin \theta^{\sharp} .
$$

Then the function $\theta^{\sharp}$ is defined by

$$
\sin \theta=\frac{G}{F}
$$

We note that

$$
\cos ^{2} \theta=\frac{\cos ^{2}{ }^{*} \theta}{\cosh ^{2} \alpha F^{2}}
$$


The important property of the coordinate $\operatorname{system}\left({ }^{*} t, r,{ }^{*} \theta,{ }^{*} \varphi\right)$ is that

$$
\dot{*} t=* \dot{\varphi}=* \dot{\theta}=0
$$

along outgoing SNG's with the correct sign of $\theta_{0}^{\prime}$. Therefore we have

$$
N^{a,+}=\frac{r^{2}+a^{2}}{\rho^{2}} k^{\prime} \partial_{r}
$$

in this coordinate system. We find

$$
\left\{\begin{array}{l}
\mathrm{d} \theta=\alpha^{\prime} \cos \theta \mathrm{d} r+\frac{\cos \theta}{\cos * \theta} \mathrm{d}^{*} \theta \\
\mathrm{d} t=\mathrm{d}^{*} t+\frac{\sigma^{2}}{\Delta B} \mathrm{~d} r+\frac{a \cos ^{2} \theta}{\cos ^{*} \theta} \mathrm{d}^{*} \theta \\
d \varphi=\mathrm{d}^{*} \varphi+\Lambda^{\prime}(r) \mathrm{d} r
\end{array}\right.
$$

Using (B.3) we can rewrite the metric as

$$
\begin{gathered}
g=\left(1+\frac{Q^{2}-2 M r}{\rho^{2}}\right) \mathrm{d}^{*} t^{2}+2 \frac{\rho^{2}}{B} \mathrm{~d}^{*} t \mathrm{~d} r \\
+2\left(1+\frac{Q^{2}-2 M r}{\rho^{2}}\right) \frac{a \cos { }^{*} \theta}{F^{2} \cosh ^{2} \alpha} \mathrm{d}^{*} t \mathrm{~d}^{*} \theta+2 a \frac{\left(2 M r-Q^{2}\right) G^{2}}{\rho^{2} F^{2}} \mathrm{~d}^{*} t \mathrm{~d}^{*} \varphi \\
-\frac{r^{2} \rho^{2} F^{2} \cosh ^{2} \alpha+a^{2}\left(2 M r-Q^{2}\right) \cos ^{2} \theta}{\rho^{2} F^{4} \cosh ^{4} \alpha} \mathrm{d}^{*} \theta^{2} \\
+2 \frac{a^{2}\left(2 M r-Q^{2}\right) G^{2} \cos ^{*} \theta}{\rho^{2} F^{4} \cosh ^{2} \alpha} \mathrm{d}^{*} \theta \mathrm{d}^{*} \varphi-\frac{\sigma^{2} G^{2}}{\rho^{2} F^{2}} \mathrm{~d}^{*} \varphi^{2} .
\end{gathered}
$$

The expression (B.4) shows that $g$ can be extended smoothly across the horizon $\left\{r=r_{+}\right\}$. Besides, it does not degenerate there since its determinant is given by

$$
\operatorname{det}(g)=-\frac{\rho^{4} \sin ^{2} \theta}{F^{2} \cosh ^{2} \alpha}
$$

and does not vanish for $r=r_{+}{ }^{(1)}$. Thus, we can add the horizon to block $I$ as a smooth boundary. It is called the past event horizon and given by

$$
\mathfrak{H}^{-}:=\mathbb{R}_{*_{t}} \times\left\{r=r_{+}\right\}_{r} \times S_{*_{\theta},{ }^{*} \varphi}^{2} .
$$

The metric induced by $g$ on hypersurfaces of constant $r, g_{r}$, has determinant

$$
\operatorname{det}\left(g_{r}\right)=-\frac{\rho^{2} \sin ^{2} \theta \Delta}{\cosh ^{2} \alpha F^{2}}
$$

and thus degenerates for $\Delta=0$, i.e. at $\mathfrak{H}^{-}$. Since $g$ does not degenerate, it follows that one of the generators of $\mathfrak{H}^{-}$is zero, i.e. $\mathfrak{H}^{-}$is a null hypersurface. Kerr-star coordinates $\left(t^{*}, r, \theta^{*}, \varphi^{*}\right)$ are constructed using the incoming SNG's:

$$
t^{*}=t+\widehat{r}(r, \theta), \quad \varphi^{*}=\varphi+\Lambda(r), \quad \theta^{*}=\theta^{\sharp}(r, \theta) .
$$

(1) Note however that there is the usual coordinate singularity at $\sin \theta=0$. 
This coordinate system allows to add the future event horizon

$$
\mathfrak{H}^{+}=\mathbb{R}_{t^{*}} \times\left\{r=r_{+}\right\} \times S_{\theta^{*}, \varphi^{*}}^{2}
$$

as a smooth null boundary to block $I$.

\section{B.2. Kruskal-Boyer-Lindquist coordinates}

The Kruskal-Boyer-Lindquist coordinate system is a combination of the two Kerr coordinate systems, modified in such a way that it is regular on both the future and the past horizons. The time and radial variables are replaced by

$$
U=\mathrm{e}^{-\kappa_{+}{ }^{*} t}, \quad V=\mathrm{e}^{\kappa_{+} t^{*}},
$$

where $\kappa_{+}$is the surface gravity at the outer horizon, see (3.5). The coordinate $\theta^{\sharp}=$ ${ }^{*} \theta=\theta^{*}$ is kept unchanged. The longitude function is defined by

$$
\varphi^{\sharp}=\varphi-\frac{a}{r_{+}^{2}+a^{2}} t .
$$

The functions $\left(U, V, \theta, \varphi^{\sharp}\right)$ form an analytic coordinate system on $\mathscr{B}_{I} \cup \mathfrak{H}^{+} \cup \mathfrak{H}^{-}-$(axes). In this coordinate system, we have

$$
\begin{gathered}
\left.\mathcal{B}_{I}=\right] 0,+\infty\left[{ }_{U} \times\right] 0,+\infty\left[{ }_{V} \times S_{\theta^{\sharp}, \varphi^{\sharp}}^{2},\right. \\
\mathfrak{H}^{+}=\{0\}_{U} \times\left[0,+\infty\left[_{V} \times S_{\theta^{\sharp}, \varphi^{\sharp}}^{2}, \quad \mathfrak{H}^{-}=\left[0,+\infty\left[U \times\{0\}_{V} \times S_{\theta^{\sharp}, \varphi^{\sharp}}^{2},\right.\right.\right.\right.
\end{gathered}
$$

simply because $t^{*}$ (resp. $\left.{ }^{*} t\right)$ is regular at $\mathfrak{H}^{+}\left(\right.$resp. $\left.\mathfrak{H}^{-}\right)$, takes all real values on $\mathfrak{H}^{+}$ $\left(\right.$ resp. $\left.\mathfrak{H}^{-}\right)$, and tends to $-\infty($ resp. $+\infty)$ at $\mathfrak{H}^{-}\left(\right.$resp. $\left.\mathfrak{H}^{+}\right)$. We want to build the crossing sphere $S_{c}^{2}=\{U=V=0\}$. To this purpose we introduce the function

$$
L:=\frac{r-r_{+}}{U V} .
$$

Lemma B.1. - (i) The functions $r$ and $\sin \theta$ are well defined analytic functions on

$$
[0, \infty)_{U} \times[0, \infty)_{V} \times S_{\theta^{\sharp}, \varphi^{\sharp}}^{2} .
$$

(ii) The function $L$ extends to a nonvanishing analytic function on

$$
[0, \infty)_{U} \times[0, \infty)_{V} \times S_{\theta^{\sharp}, \varphi^{\sharp}}^{2} .
$$

Proof. - (i) We have

$$
\left(\begin{array}{c}
U V \\
\sin \theta^{\sharp}
\end{array}\right)=\left(\begin{array}{c}
\mathrm{e}^{2 \kappa_{+} \widehat{r}(r, \theta)} \\
\frac{\tanh (\alpha)-\sin \theta}{\tanh (\alpha) \sin \theta-1}
\end{array}\right)=H(r, \sin \theta)=\left(\begin{array}{c}
H_{U V}(r, \sin \theta) \\
H_{\theta^{\sharp}}(r, \sin \theta)
\end{array}\right) .
$$

Using

$$
\mathrm{e}^{2 \kappa_{+} \widehat{r}}=\left(r-r_{+}\right)\left(r-r_{-}\right)^{\frac{\kappa_{+}}{\kappa_{-}}} \exp \left(2 \kappa_{+} \int_{r_{+}}^{r}\left(\sqrt{1-\frac{a^{2} \Delta}{\left(r^{2}+a^{2}\right)^{2}}}-1\right) \frac{\tau^{2}+a^{2}}{\Delta} \mathrm{d} \tau\right) \mathrm{e}^{2 \kappa_{+} a \sin \theta}
$$


we easily check that

$$
\left(\begin{array}{ll}
\partial_{1} H_{U V} & \partial_{2} H_{U V} \\
\partial_{1} H_{\theta \sharp} & \partial_{2} H_{\theta \sharp}
\end{array}\right)\left(r_{+}, \sin \theta\right)
$$

is invertible. Thus $(r, \sin \theta)=H^{-1}\left(U V, \sin \theta^{\sharp}\right)$ is well defined in a neighborhood of the crossing sphere.

(ii) We have

$$
L=\left(r-r_{-}\right)^{-\frac{\kappa_{+}}{\kappa_{-}}} \exp \left(-2 \kappa_{+} \int_{r_{+}}^{r}\left(\sqrt{1-\frac{a^{2} \Delta}{\left(r^{2}+a^{2}\right)^{2}}}-1\right) \frac{\tau^{2}+a^{2}}{\Delta} \mathrm{d} \tau\right) \mathrm{e}^{-2 \kappa_{+} a \sin \theta} .
$$

This and the result of (i) give (ii).

We have

$$
\left\{\begin{array}{l}
\mathrm{d} t=\frac{L}{2 \kappa_{+}\left(r-r_{+}\right)}(U \mathrm{~d} V-V \mathrm{~d} U), \\
\mathrm{d} r=\frac{\left(r-r_{-}\right) L B}{2 \kappa_{+} \sigma^{2}}(U \mathrm{~d} V+V \mathrm{~d} U)-\frac{B \Delta}{\sigma^{2}} \frac{a \cos ^{2} \theta}{\cos \theta^{\sharp}} \mathrm{d} \theta^{\sharp}, \\
\mathrm{d} \varphi=\mathrm{d} \varphi^{\sharp}+\frac{a}{r_{+}^{2}+a^{2}} \frac{L}{2 \kappa_{+}\left(r-r_{+}\right)}(U \mathrm{~d} V-V \mathrm{~d} U), \\
\mathrm{d} \theta=\frac{a\left(r-r_{-}\right) \cos \theta L}{2 \kappa_{+} \sigma^{2}}(U \mathrm{~d} V+V \mathrm{~d} U)+\frac{\cos \theta B^{2}}{\cos \theta^{\sharp} \sigma^{2}} \mathrm{~d} \theta^{\sharp} .
\end{array}\right.
$$

Using (B.7) we find that the Kerr-Newman metric in these coordinates takes the form

$$
\begin{gathered}
g=-\frac{2 L\left(r-r_{-}\right)}{4 \kappa_{+}^{2} \rho^{2}}\left(\frac{\rho_{+}^{4}}{\left(r_{+}^{2}+a^{2}\right)^{2}}+\frac{\rho^{4} B^{2}}{\sigma^{4}}\right) \mathrm{d} U \mathrm{~d} V \\
+\frac{L^{2}\left(r-r_{-}\right)}{4 \kappa_{+}^{2} \rho^{2}\left(r-r_{+}\right)}\left(\frac{\rho_{+}^{4}}{\left(r_{+}^{2}+a^{2}\right)^{2}}-\frac{\rho^{4} B^{2}}{\sigma^{4}}\right)\left(V^{2} \mathrm{~d} U^{2}+U^{2} \mathrm{~d} V^{2}\right) \\
-\rho^{2} \frac{a^{2}\left(r-r_{-}\right)^{2} \cos ^{2} \theta L^{2}}{4 \kappa_{+}^{2} \sigma^{4}}(U \mathrm{~d} V+V \mathrm{~d} U)^{2} \\
-\frac{\sin ^{2} \theta}{\rho^{2}} \frac{\left(r+r_{+}\right)^{2} a^{2} L^{2}}{\left(r_{+}-r_{-}\right)^{2}}(U \mathrm{~d} V-V \mathrm{~d} U)^{2} \\
-\frac{\sin ^{2} \theta a L}{\kappa_{+} \rho^{2}\left(r_{+}^{2}+a^{2}\right)}\left(\left(r-r_{-}\right) \rho_{+}^{2}+\left(r^{2}+a^{2}\right)\left(r+r_{+}\right)\right)(U \mathrm{~d} V-V \mathrm{~d} U) \mathrm{d} \varphi^{\sharp} \\
-\frac{\rho^{2} B^{2}}{\sigma^{2} \cosh ^{2} \alpha F^{2}}\left(\mathrm{~d} \theta^{\sharp}\right)^{2}-\frac{\sigma^{2} \sin ^{2} \theta}{\rho^{2}}\left(\mathrm{~d} \varphi^{\sharp}\right)^{2},
\end{gathered}
$$

where $\rho_{+}^{2}=r_{+}^{2}+a^{2}$. Clearly $1 /\left(r-r_{+}\right)\left(\rho_{+}^{4} /\left(r_{+}^{2}+a^{2}\right)^{2}-\rho^{4} B^{2} / \sigma^{4}\right)$ extends to an analytic function on $[0, \infty)_{U} \times[0, \infty)_{V} \times S_{\theta^{\sharp}, \varphi^{\sharp}}^{2}$. The expression (B.8) then shows that $g$ is smooth on $\mathscr{B}_{I} \cup \mathfrak{H}^{+} \cup \mathfrak{H}^{-}$and can be extended smoothly on 
$\left[0,+\infty\left[_{U} \times\left[0,+\infty\left[\left[_{V} \times S_{\theta^{\sharp}, \varphi^{\sharp}}^{2}\right.\right.\right.\right.\right.$. The crossing sphere $S_{c}^{2}=\{U=V=0\}$ is a regular surface in the extended space-time

$$
\left(\mathcal{B}_{I}^{\mathrm{KBL}}:=\left[0,+\infty\left[U \times\left[0,+\infty\left[V \times S_{\theta^{\sharp}, \varphi^{\sharp}}^{2}, g\right) .\right.\right.\right.\right.
$$

Hence, the Kruskal-Boyer-Lindquist coordinates give us a global description of the horizon

$$
\mathfrak{H}=\mathfrak{H}^{-} \cup S_{c}^{2} \cup \mathfrak{H}^{+}=\left(\left[0,+\infty\left[[ _ { U } \times \{ 0 \} _ { V } \times S _ { \theta ^ { \sharp } , \varphi ^ { \sharp } } ^ { 2 } ) \cup \left(\{0\}_{U} \times\left[0,+\infty\left[{ }_{V} \times S_{\theta^{\sharp}, \varphi^{\sharp}}^{2}\right)\right.\right.\right.\right.\right.
$$

as a union of two smooth null boundaries $\mathfrak{H}^{+} \cup S_{c}^{2}$ and $S_{c}^{2} \cup \mathfrak{H}^{-}$.

\section{B.3. Penrose compactification of Block $I$}

The Penrose compactification of the exterior of a Kerr-Newman black hole is performed using two independent and symmetric constructions, one based on star-Kerr, the other on Kerr-star coordinates. We describe explicitly only the first of these two constructions.

Future null infinity is defined as the set of limit points of outgoing simple null geodesics as $r \rightarrow+\infty$. This rather abstract definition of a 3 -surface, describing the congruence of outgoing simple null geodesics, can be given a precise meaning using star-Kerr coordinates. We consider the expression (B.4) of the Kerr-Newman metric in star-Kerr coordinates and replace the variable $r$ by $w=1 / r$. In these new variables, the exterior of the black hole is described as

$$
\left.\mathcal{B}_{I}=\mathbb{R}_{{ }^{*} t} \times\right] 0, \frac{1}{r_{+}}\left[_{w} \times S_{{ }^{*} \theta,{ }^{*} \varphi}^{2} .\right.
$$

The conformally rescaled metric

$$
\widehat{g}=\Omega^{2} g, \quad \Omega=w=\frac{1}{r}
$$

takes the form

$$
\begin{aligned}
\widehat{g}= & \left(w^{2}+\frac{Q^{2} w^{4}-2 M w^{3}}{1+a^{2} w^{2} \cos ^{2} \theta}\right) \mathrm{d}^{*} t^{2} \\
- & 2 \frac{1+a^{2} w^{2} \cos ^{2} \theta}{\sqrt{\left(1+a^{2} w^{2}\right)^{2}-a^{2} w^{2}\left(1+\left(a^{2}+Q^{2}\right) w^{2}-2 M w\right)}} \mathrm{d}^{*} t \mathrm{~d} w \\
& +2 w^{2}\left(1+\frac{Q^{2} w^{2}-2 M w}{1+a^{2} w^{2} \cos ^{2} \theta}\right) \frac{a \cos ^{*} \theta}{F^{2} \cosh ^{2} \alpha} \mathrm{d}^{*} t \mathrm{~d}^{*} \theta \\
& +2 a w^{3} \frac{\left(2 M-Q^{2} w\right) G^{2}}{\left(1+a^{2} \cos ^{2} \theta w^{2}\right) F^{2}} \mathrm{~d}^{*} t \mathrm{~d}^{*} \varphi
\end{aligned}
$$




$$
\begin{aligned}
& -\frac{\left(1+a^{2} \cos ^{2} \theta w^{2}\right) F^{2} \cosh ^{2} \alpha+a^{2}\left(2 M w^{3}-Q^{2} w^{4}\right) \cos ^{2} \theta}{\left(1+a^{2} \cos ^{2} \theta w^{2}\right) F^{4} \cosh ^{4} \alpha} d^{*} \theta^{2} \\
& +2 a w^{3} \frac{\left(2 M-Q^{2} w\right) G^{2} \cos ^{*} \theta}{\left(1+a^{2} \cos ^{2} \theta w^{2}\right) \cosh ^{2} \alpha F^{4}} \mathrm{~d}^{*} \theta \mathrm{d}^{*} \varphi \\
& -\frac{\left(\left(1+a^{2} w^{2}\right)^{2}-a^{2} w^{2}\left(1+\left(a^{2}+Q^{2}\right) w^{2}-2 M w\right) \sin ^{2} \theta\right) G^{2}}{\left(1+a^{2} \cos ^{2} \theta w^{2}\right) F^{2}} \sin ^{2} \theta \mathrm{d}^{*} \varphi^{2} .
\end{aligned}
$$

The functions $\sin \theta, \cos \theta, F, G, \alpha$ have to be understood as functions of $1 / w,{ }^{*} \theta$. It is clear from the formulas in Section B.1 that they possess analytic extensions to $\left[0,1 / r_{+}\right] \times S_{* \theta}^{2}$. The expression above shows that $\widehat{g}$ can be extended smoothly on the domain

$$
\mathbb{R}_{* t} \times\left[0, \frac{1}{r_{+}}\right]_{w} \times S_{* \theta,{ }^{*} \varphi}^{2}
$$

The hypersurface

$$
\mathcal{J}^{+}:=\mathbb{R}_{* t} \times\{w=0\} \times S_{* \theta,{ }^{*} \varphi}^{2}
$$

can thus be added to the rescaled space-time as a smooth hypersurface, describing future null infinity as defined above. This hypersurface is indeed null since

$$
\widehat{g}_{\left.\right|_{w=0}}=-\mathrm{d}^{*} \theta^{2}-\sin ^{2} \theta \mathrm{d}^{*} \varphi^{2}
$$

is degenerate (recall that $\mathrm{J}^{+}$is a 3 -surface) and

$$
\operatorname{det}(\widehat{g})=-\frac{\left(1+a^{2} w^{2} \cos ^{2} \theta\right)^{2}}{F^{2} \cosh ^{2} \alpha} \sin ^{2} \theta
$$

does not vanish for $w=0$.

Similarly, using Kerr-star instead of star-Kerr coordinates, we define past null infinity, the set of limit points as $r \rightarrow+\infty$ of incoming simple null geodesics, as

$$
\mathcal{J}^{-}:=\mathbb{R}_{t^{*}} \times\{w=0\} \times S_{\theta^{*}, \varphi^{*}}^{2}
$$

The Penrose compactification of block $I$ is then the space-time

$$
\left(\overline{\mathcal{B}_{I}}, \widehat{g}\right), \quad \overline{\mathcal{B}_{I}}=\mathscr{B}_{I} \cup \mathfrak{H}^{+} \cup S_{\mathrm{c}}^{2} \cup \mathfrak{H}^{-} \cup \mathcal{J}^{+} \cup \mathcal{J}^{-},
$$

$\widehat{g}$ being defined by (B.9). In spite of the terminology used, the compactified spacetime is not compact. There are three "points" missing to the boundary: $i_{+}$, or future timelike infinity, defined as the limit point of uniformly timelike curves as $t \rightarrow+\infty, i_{-}$, past timelike infinity, symmetric of $i_{+}$in the distant past, and $i_{0}$, spacelike infinity, the 


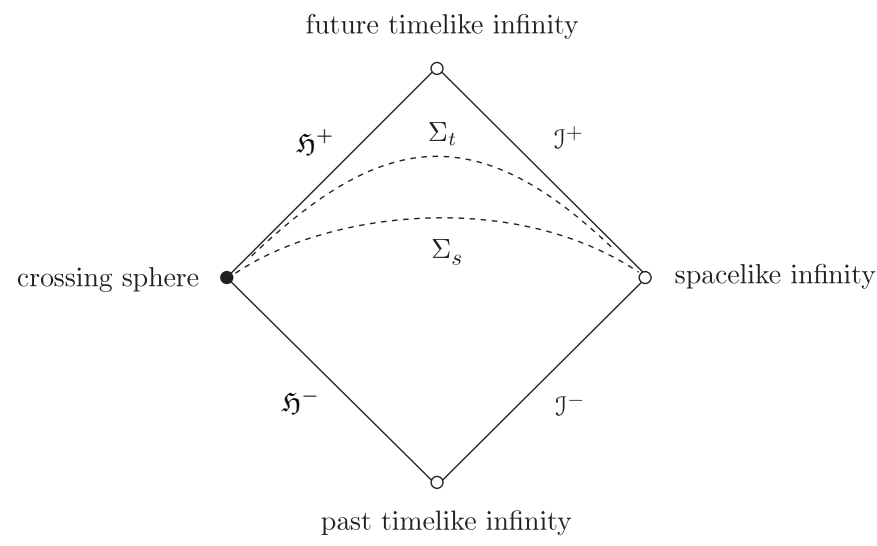

Figure 1. The Penrose compactification of block $I$, with two hypersurfaces $\Sigma_{s}$ and $\Sigma_{t}, t>s$.

limit point of uniformly spacelike curves as $r \rightarrow+\infty$. These "points" are singularities of the rescaled metric. See Figure 1 for a representation of the compactified block $I$.

Writing

$$
U=\mathrm{e}^{-\kappa_{+}(t-\widehat{z}(t, \theta))}, \quad V=\mathrm{e}^{\kappa_{+}(t+\widehat{z}(t, \theta))}
$$

and suppressing two dimensions gives the picture of the collapse of Figure 1, Section 4.5. 



\section{BIBLIOGRAPHY}

[1] A. BACHELOT - "Gravitational scattering of electromagnetic field by a Schwarzschild black hole", Ann. Inst. Henri Poincaré, Phys. Théor. 54 (1991), p. 261-320.

[2] _ _ "Asymptotic completeness for the Klein-Gordon equation on the Schwarzschild metric", Ann. Inst. Henri Poincaré, Phys. Théor. 61 (1994), p. 411-441.

[3] _ "Quantum vacuum polarization at the black-hole horizon", Ann. Inst. Henri Poincaré, Phys. Théor. 67 (1997), p. 181-222.

[4] _ , "Scattering of scalar fields by spherical gravitational collapse", J. Math. Pures Appl. 76 (1997), p. 155-210.

[5] _ "The Hawking effect", Ann. Inst. Henri Poincaré, Phys. Théor. 70 (1999), p. 41-99.

[6] __ "Creation of fermions at the charged black-hole horizon", Ann. Henri Poincaré 1 (2000), p. 1043-1095.

[7] _ "Superradiance and scattering of the charged Klein-Gordon field by a step-like electrostatic potential", J. Math. Pures Appl. 83 (2004), p. 1179-1239.

[8] A. N. Bernal \& M. SANChEZ - "On smooth hypersurfaces and Geroch's splitting theorem", Comm. Math. Phys. 243 (2003), p. 461-470.

[9] _ _ "Smoothness of time functions and the metric splitting of globally hyperbolic space-times", Comm. Math. Phys. 257 (2005), p. 43-50.

[10] S. DE BiÈvre, P. Hislop \& I. Sigal - "Scattering theory for the wave equation on non-compact manifolds", Rev. Math. Phys. 4 (1992), p. 575-618.

[11] O. Bratelli \& D. W. Robinson - Operator algebras and quantum statistical mechanics 2, Springer Verlag, 1997.

[12] B. CARTER - Black hole equilibrium states, black holes/les astres occlus, École d'été de Physique théorique, Les Houches, 1972, pp. 57-214, Gordon and Breach, 1973. 
[13] S. Chandrasekhar - The mathematical theory of black holes, Oxford Univ. Press, 1983.

[14] T. DAudÉ - "Scattering of charged Dirac fields by a ReissnerNordström black hole", Preprint Université Bordeaux 1, 2004, Ph.D. thesis Université Bordeaux 1 (2004), Chapter 3, available online at http://tel .archives-ouvertes.fr/tel-00011974/en/.

[15] _ "Scattering of charged Dirac fields by a Kerr-Newman black hole", Preprint Université Bordeaux 1, 2004, Ph.D. thesis Université Bordeaux 1 (2004), Chapter 4, available online at http://tel .archives-ouvertes.fr/tel-00011974/en/.

[16] J. Dimock - "Algebras of local observables on a manifold", Comm. Math. Phys. 77 (1980), p. 219-228.

[17] _ _Dirac quantum fields on a manifold", Trans. Amer. Math. Soc. 269 (1982), p. 133-147.

[18] _ "Scattering for the wave equation on the Schwarzschild metric", Gen. Relativ. Gravitation 17 (1985), p. 353-369.

[19] J. Dimock \& B. S. KAY - "Scattering for massive scalar fields on Coulomb potentials and Schwarzschild metrics", Classical Quantum Gravity 3 (1986), p. 7180 .

[20] _ "Classical and quantum scattering theory for linear scalar fields on the Schwarzschild metric I", Ann. Phys. 175 (1987), p. 366-426.

[21] _ "Classical and quantum scattering theory for linear scalar fields on the Schwarzschild metric II", J. Math. Phys. 27 (1986), p. 2520-2525.

[22] F. Finster, N. Kamran, J. Smoller \& S.-T. Yau - "An integral spectral representation of the propagator for the wave equation in the Kerr geometry", Comm. Math. Phys. 260 (2005), p. 257-298.

[23] S. J. Flechter \& A. W. C. Lun - "The Kerr spacetime in generalized BondiSachs coordinates", Class. Quantum Grav. 20 (2003), p. 4153-4167.

[24] R. P. GEROCH - "Spinor structure of space-times in general relativity I", J. Math. Phys. 9 (1968), p. 1739-1744.

[25] _ "Spinor structure of space-times in general relativity II", J. Math. Phys. 11 (1970), p. 342-348.

[26] _ "The domain of dependence", J. Math. Phys. 11 (1970), p. 437-449. 
[27] D. HÄFNER - "Complétude asymptotique pour l'équation des ondes dans une classe d'espaces-temps stationnaires et asymptotiquement plats", Ann. Inst. Fourier 51 (2001), p. 779-833.

[28] _ "Sur la théorie de la diffusion pour l'équation de Klein-Gordon dans la métrique de Kerr", Dissertationes Mathematicae 421 (2003), 102 pp.

[29] D. HÄFner \& J.-P. NicOlas - "Scattering of massless Dirac fields by a Kerr black hole", Rev. Math. Phys. 16 (2004), p. 29-123.

[30] S. W. Hawking - "Particle creation by black holes", Comm. Math. Phys. 43 (1975), p. 199-220.

[31] L. Hörmander - The Analysis of Linear Partial Differential Operators, Vol. $I-I V$, Springer, 1985.

[32] _ "A remark on the characteristic Cauchy problem", J. Funct. Anal. 93 (1990), p. 270-277.

[33] W. M. JiN - "Scattering of massive Dirac fields on the Schwarzschild black hole spacetime", Classical Quantum Gravity 15 (1998), p. 3163-3175.

[34] W. Kinnersley - "Type D vacuum metrics", J. Math. Phys. 10 (1969), p. 11951203.

[35] L. J. MAson \& J.-P. NiCOLAS - "Conformal scattering and the Goursat problem", J. Hyperbolic Differ. Equ. 1 (2004), p. 197-233.

[36] F. MELnYK - "Scattering on Reissner-Nordstrøm metric for massive charged spin $\frac{1}{2}$ fields", Ann. Henri Poincaré 4 (5) (2003), p. 813-846.

[37] _ "The Hawking effect for spin $\frac{1}{2}$ fields", Comm. Math. Phys. 244 (2004), p. $483-525$.

[38] J.-P. NiCOLAS - "Scattering of linear Dirac fields by a spherically symmetric black hole", Ann. Inst. Henri Poincaré, Phys. Théor. 62 (1995), p. 145-179.

[39] _ "Global exterior Cauchy problem for spin $\frac{3}{2}$ zero rest-mass fields in the Schwarzchild space-time", Comm. Partial Differential Equations 22 (1997), p. 465-502.

[40] _ _ "Dirac fields on asymptotically flat space-times", Dissertationes Mathematicae 408 (2002), 85 pp.

[41] _ "On Lars Hörmander's remark on the characteristic Cauchy problem", Ann. Inst. Fourier 56 (2006), p. 517-543.

[42] B. O'NeILL - The geometry of Kerr black holes, A.K. Peters, Wellesley, 1995. 
[43] A. PAZY - Semigroups of linear operators and applications to partial differential equations, Applied Mathematical Sciences, vol. 44, Springer Verlag, 1983.

[44] R. Penrose \& W. Rindler - Spinors and space-time, vol. I, Cambridge monographs on mathematical physics, Cambridge Univ. Press, 1984.

[45] E. Stiefel - "Richtungsfelder und Fernparallelismus in $n$-dimensionalen Mannigfaltigkeiten", Comment. Math. Helv. 8 (1936), p. 305-353.

[46] B. Thaller - The Dirac equation, Texts and monographs in mathematical physics, Springer Verlag, 1992.

[47] R. M. WALD - "On particle creation by black holes", Comm. Math. Phys. 45 (1975), p. 9-34.

[48] _ General relativity, The University of Chicago Press, 1984. 